\author{
UNIVERSIDADE DE SÃO PAULO \\ ESCOLA DE ENGENHARIA DE SÃO CARLOS \\ DEPARTAMENTO DE ENGENHARIA ELÉTRICA
}

\title{
Extensão do Método das Diferenças Finitas para o Projeto e Modelagem de Dispositivos Ópticos Utilizando Meios com Propriedades Diversas
}

\section{Licinius Dimitri Sá de Alcantara}

Tese apresentada à Escola de Engenharia de São Carlos, da Universidade de São Paulo, como parte dos requisitos para obtenção do título de Doutor em Engenharia Elétrica.

Orientador: Prof. Dr. Ben-Hur Viana Borges

Co-Orientador: Prof. Dr. Amilcar Careli César

São Carlos, SP

2004 

Aos meus pais, professores e amigos. 


\section{AGRADECIMENTOS}

Aos meus pais, Lélio e Elizabet, pelo apoio e atenção que supera a distância.

Ao meu orientador Prof. Dr. Ben-Hur Viana Borges, pelo incentivo e dedicação inesgotável.

Ao Prof. Dr. Amílcar Careli César, meu co-orientador, e ao Prof. Dr. Fernando Lisboa Teixeira, pelas importantes sugestões e acompanhamento deste trabalho.

Aos colegas de mestrado e doutorado da EESC/USP pela convivência e a oportunidade de compartilhar experiências e conhecimentos.

Aos demais familiares e amigos.

Aos Professores e funcionários do Departamento de Engenharia Elétrica da EESC/USP.

À FAPESP, pelo suporte a este trabalho. 


\section{SUMÁRIO}

LISTA DE FIGURAS ...................................................................................................

LISTA DE TABELAS ............................................................................................ v

LISTA DE ABREVIATURAS E SIGLAS................................................................ vii

LISTA DE SÍMBOLOS............................................................................................viii

RESUMO ……................................................................................................................. ix

ABSTRACT ....................................................................................................................

1 INTRODUÇÃ O...................................................................................................... 1

1.1 O MÉTODO DAS DIFERENÇAS FINITAS NO DOMÍNIO DO TEMPO (FDTD) .......... 1

1.2 O MÉTODO DA PROPAGAÇÃO DE FEIXE (BPM) ……………………............

1.3 CONTRIBUIÇÕES DESTA TESE ………………………..............................

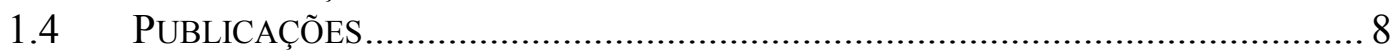

1.5 ORGANIZAÇÃO DO TEXTO ..................................................................... 10

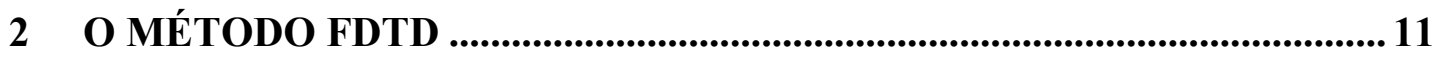

2.1 DISCRETIZAÇÃO TEMPORAL DAS EQUAÇÕES DE MAXWELL............................. 11

2.2 DISCRETIZAÇÃO ESPACIAL DAS EQUAÇÕES DE MAXWELL ………………...... 12

2.2.1 Discretização espacial usando célula de Yee ………………………...... 12

2.2.2 Discretização espacial usando célula de dois nós................................13

2.3 CRITÉRIOS PARA PRECISÃO E ESTABILIDADE ……………............................... 15

2.4 TRANSFORMADA DISCRETA DE FOURIER (DFT) .......................................... 16

2.5 CONDIÇÕES DE CONTORNO NA BORDA DA JANELA COMPUTACIONAL ........... 17

2.6 CASO PARTICULAR: GUIA PLANAR NÃO-LINEAR E NÃO-RECÍPROCO .............. 23

3 O MÉTODO FD-BPM BIDIMENSIONAL ................................................... 29

3.1 EXPANSÃO DO FORMALISMO FD-BPM ………………………………..... 29

3.1.1 Implementação do esquema Crank-Nicolson (CN)..................................33

3.1.2 Implementação do esquema Douglas Generalizado (GD) ..................... 34

3.2 MÉTODO ITERATIVO ENTRE O CAMPO ELÉTRICO E A PERMISSIVIDADE

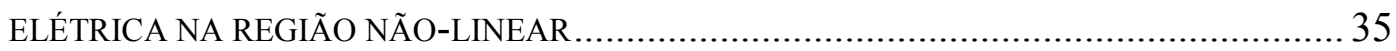

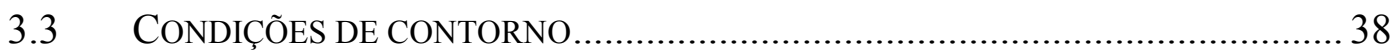

3.3.1 Camada Perfeitamente Casada ou PML (Perfectly Matched Layer) .... 38

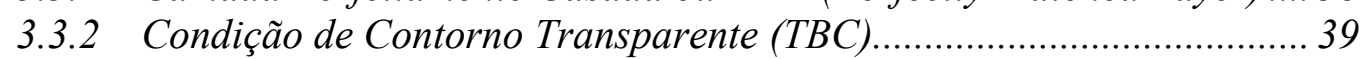


4 MÉTODO FD-BPM TRIDIMENSIONAL VETORIAL BASEADO NA TÉCNICA ADI ................................................................................... 40

4.1 EXPANSÃO DO FORMALISMO FD-BPM …............................................. 40

4.1.1 FD-BPM para o cálculo da componente $x$ do vetor campo elétrico ..... 45

4.1.2 FD-BPM para a componente y do vetor campo elétrico ....................... 48

5 EXEMPLOS BIDIMENSIONAIS E RESULTADOS ....................................51

5.1 GUIA PLANAR NÃO-LINEAR E NÃO-RECÍPROCO........................................ 51

5.2 ACOPLADOR DIRECIONAL NÃO-LINEAR E NÃO-RECÍPROCO............................55

5.3 EFEITO DA NÃO-RECIPROCIDADE SOBRE A EMISSÃO DE SÓLITONS................. 61

6 EXEMPLOS TRIDIMENSIONAIS E RESULTADOS .............................. 64

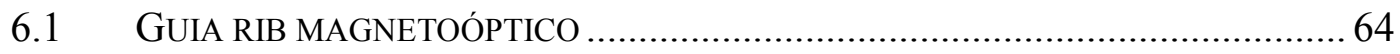

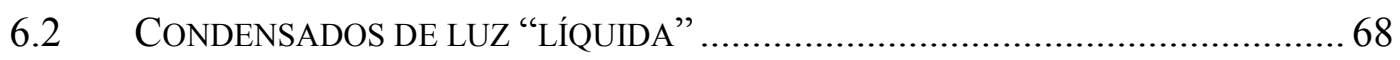

6.2.1 Estabilização de um feixe gaussiano................................................... 71

6.2.2 Colisão de um feixe supergaussiano em uma interface com o ar.......... 74

6.2.3 Colisão entre dois feixes ópticos........................................................... 86

6.2.4 Passagem de um feixe condensado através de uma fenda................... 95

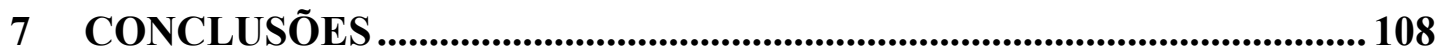

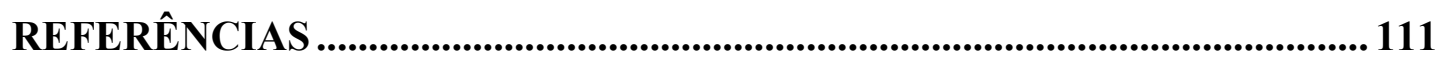

APÊNDICE A - Algoritmo do Método FDTD ........................................................ 118

APÊNDICE B - Algoritmo do FD-BPM bidimensional....................................... 119

APÊNDICE C - Algoritmo do FD-BPM 3D vetorial......................................... 120

APÊNDICE D - Conversão entre simbolismos não-lineares ............................... 122

APÊNDICE E - Teste de precisão do método FD-BPM 2D................................. 124

APÊNDICE F - Teste do método FD-BPM 3D vetorial....................................... 126 


\section{LISTA DE FIGURAS}

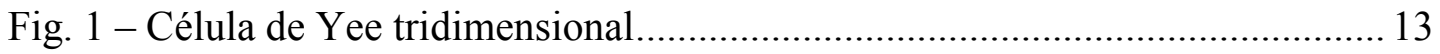

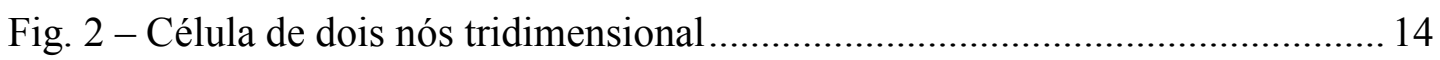

Fig. 3 - Disposições das camadas absorventes (GMIPML) num problema

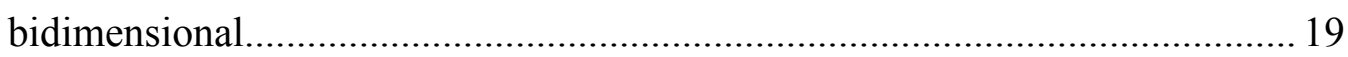

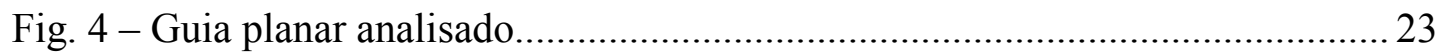

Fig. 5 - Célula de Yee bidimensional (plano $x z$ ) ....................................................... 24

Fig. 6 - Discretização espacial ao longo da direção transversal, x.......................... 26

Fig. 7 - Onda incidindo no limite frontal após 500 passos de tempo: (a) na ausência de GMIPML $(\mathrm{Npml}=0)$ e (b) com o domínio revestido pelas camadas absorventes $\left(n p m l=30, p=3\right.$ e $\left.R(0)=10^{-2}\right)$..................................... 28

Fig. 8 - Índice efetivo modal em função da potência de entrada do laser. .................53

Fig. 9 - Desvio de fase não-recíproco em função da potência de entrada do laser.

Fig. 10 - Comportamento modal em função do aumento da densidade de potência de entrada: (a) distribuição de $\left|H_{y}\right|$ para $\mathrm{P}_{\mathrm{exc}}=125 \mathrm{~mW} / \mathrm{mm}$ (linhas brancas delimitam o filme linear) e (b) índice efetivo em função da direção longitudinal $\mathrm{z}$

Fig. 11 - Canal induzido no meio não-linear para uma densidade de potência de entrada de $303 \mathrm{~mW} / \mathrm{mm}$. (a) Condição de contorno transparente, TBC, (b)-(c) Condição de contorno de camadas perfeitamente casadas, PML. As linhas centrais indicam a localização do filme guia de onda.

Fig. 12 - Esboço inicial do dispositivo chave/isolador. A faixa de interação entre os guias é delimitada por um ângulo de inclinação de $\theta=1,791^{\circ} \mathrm{e}$ o comprimento de onda de operação é $\lambda=1,32 \mu m$.

Fig. 13 - Índice efetivo em função da densidade de potência óptica de excitação para o guia não-linear e não-recíproco isolado (guia $\mathrm{A}$ ). 
Fig. 14 - Fluxo de intensidade de campo para: (a) $\mathrm{P} 1=1,0 \mathrm{~mW} / \mathrm{mm}$ e propagação $+z$, (b) $\mathrm{P} 1=1,0 \mathrm{~mW} / \mathrm{mm}$ e propagação $-z$., (c) $\mathrm{P} 2=23,0$ $\mathrm{mW} / \mathrm{mm}$ e propagação $+z$, (d) $\mathrm{P} 2=23,0 \mathrm{~mW} / \mathrm{mm}$ e propagação $-z$. Compare com o diagrama da Tabela 5 .

Fig. 15 - Guia planar não-linear e não-recíproco.

Fig. 16 - Efeito do valor da constante magnetoóptica $\delta$ sobre a emissão de sóliton para $P_{\text {exc }}=280 \mathrm{~mW} / \mathrm{mm}$.

Fig. 17 - Variação do índice efetivo em função da distância de propagação correspondente às distribuições de campo mostradas na Fig. 16.

Fig. 18 - Variação do índice efetivo em função da distância de propagação para valor absoluto de $\delta$ dez vezes menor em relação à Fig. 17. 63

Fig. 19 - Guia rib magnetoóptico.

Fig. 20 - Distribuições transversais de campo para as componentes $E_{x}$ (coluna à esquerda), $E_{y}$ (coluna central) e $E_{z}$ (coluna à direita) em $\mathrm{z}=0 \mu \mathrm{m}$, $1700 \mu \mathrm{m}, 3400 \mu \mathrm{m}, 5100 \mu \mathrm{m}$, e $6800 \mu \mathrm{m}$.

Fig. 21 - Evolução da intensidade normalizada das componentes transversais de campo elétrico ao longo do eixo de propagação do guia magnetoóptico.

Fig. 22 - Variação da permissividade elétrica relativa com o módulo do campo elétrico no meio não-linear. $\mathrm{O}$ ponto $\mathbf{A}$ indica o estado de saturação.

Fig. 23 - Transição de um pulso gaussiano de alta intensidade (visto em $z=0$ ) a um formato supergaussiano (visto em $z=8100 \mu \mathrm{m}$ ) em um meio do tipo Kerr com saturação.

Fig. 24 - Distribuição de índice de refração dependente da intensidade de campo elétrico, devido à propagação da supergaussiana mostrada na Fig. $3(\mathrm{z}=8100 \mu \mathrm{m})$. O núcleo côncavo surge como uma peculiaridade do meio não-linear cúbico-quíntico.

Fig. 25 - Componentes $E_{x}, E_{y}$ e $E_{z}$ da supergaussiana mostrada à direita da

Fig. $23(\mathrm{z}=8100 \mu \mathrm{m})$. 
Fig. 26 - Esboço da simulação realizada no meio não-linear com saturação, onde um feixe óptico é totalmente refletido após incidir na interface com o ar.

Fig. 27 - Choque de um feixe óptico condensado em uma interface com o ar para um ângulo de incidência de $2^{\circ}$, componente $E_{x}$

Fig. 28 - Observação da evolução da componente $E_{y}$ referente ao mesmo exemplo da Fig. 27.

Fig. 29 - Observação da evolução da componente $E_{z}$ referente ao mesmo exemplo da Fig. 27.

Fig. 30 - Componente dominante $E_{x}$ referente ao choque de um feixe óptico condensado em uma interface com o ar para um ângulo de incidência de $3^{\circ}$.

Fig. 31 - Componente $E_{y}$ referente ao choque de um feixe óptico condensado em uma interface com o ar para um ângulo de incidência de $3^{\circ}$.

Fig. 32 - Componente $E_{z}$ referente ao choque de um feixe óptico condensado em uma interface com o ar para um ângulo de incidência de $3^{\circ}$.

Fig. 33 - Componente dominante $E_{x}$ referente ao choque de um feixe óptico condensado em uma interface com o ar para um ângulo de incidência de $4^{\mathrm{o}}$

Fig. 34 -Componente $E_{y}$ referente ao choque de um feixe óptico condensado em uma interface com o ar para um ângulo de incidência de $4^{\circ}$.

Fig. 35 - Componente $E_{z}$ referente ao choque de um feixe óptico condensado em uma interface com o ar para um ângulo de incidência de $4^{\circ}$.

Fig. 36 - Componente dominante $E_{x}$ referente ao choque entre feixes ópticos em fase para um ângulo de propagação de $3^{\circ}$ em relação ao plano horizontal.

Fig. 37 - Componente $E_{y}$ referente ao choque entre feixes ópticos em fase para um ângulo de propagação de $3^{\circ}$ em relação ao plano horizontal. 90

Fig. 38 - Componente $E_{z}$ referente ao choque entre feixes ópticos em fase para um ângulo de propagação de $3^{\circ}$ em relação ao plano horizontal. 
Fig. 39 - Componente dominante $E_{x}$ referente ao choque entre feixes ópticos defasados de $180^{\circ}$ para um ângulo de propagação de $3^{\circ}$ em relação ao plano horizontal.

Fig. 40 - Componente $E_{y}$ referente ao choque entre feixes ópticos defasados de $180^{\circ}$ para um ângulo de propagação de $3^{\circ}$ em relação ao plano horizontal.

Fig. 41 - Componente $E_{z}$ referente ao choque entre feixes ópticos defasados de $180^{\circ}$ para um ângulo de propagação de $3^{\circ}$ em relação ao plano horizontal.

Fig. 42 - Ilustração da fenda metálica inserida no meio não-linear. .95

Fig. 43 - Componente dominante $E_{x}$ referente à incidência de um feixe óptico numa fenda metálica de $1,0 \mu \mathrm{m}$ de abertura. 98

Fig. 44 - Componente minoritária $E_{y}$ referente à incidência de um feixe óptico numa fenda metálica de $1,0 \mu \mathrm{m}$ de abertura.

Fig. 45 - Componente minoritária $E_{z}$ referente à incidência de um feixe óptico numa fenda metálica de $1,0 \mu \mathrm{m}$ de abertura. 100

Fig. 46 - Componente dominante $E_{x}$ referente à incidência de um feixe óptico numa fenda metálica de $2,5 \mu \mathrm{m}$ de abertura.

Fig. 47 -Componente minoritária $E_{y}$ referente à incidência de um feixe óptico numa fenda metálica de $2,5 \mu \mathrm{m}$ de abertura.

Fig. 48 - Componente minoritária $E_{z}$ referente à incidência de um feixe óptico numa fenda metálica de $2,5 \mu \mathrm{m}$ de abertura.

Fig. 49 - Componente dominante $E_{x}$ referente à incidência de um feixe óptico numa fenda metálica de 5,0 $\mu \mathrm{m}$ de abertura.

Fig. 50 - Componente minoritária $E_{y}$ referente à incidência de um feixe óptico numa fenda metálica de 5,0 $\mu \mathrm{m}$ de abertura.

Fig. 51 - Componente minoritária $E_{z}$ referente à incidência de um feixe óptico numa fenda metálica de 5,0 $\mu \mathrm{m}$ de abertura. 106

Fig. 52 - Evolução da intensidade de campo elétrico em função da distância de propagação. A fenda está situada em z=500 $\mathrm{m}$. 


\section{LISTA DE TABELAS}

TABELA 1 - Propriedades das regiões no contexto da GMIPML ............................. 19

TABELA 2 - Parâmetros dos materiais .................................................................... 27

TABELA 3 - Elementos da matriz permissividade inversa. ..................................... 30

TABELA 4 - Parâmetros utilizados nas simulações ................................................ 51

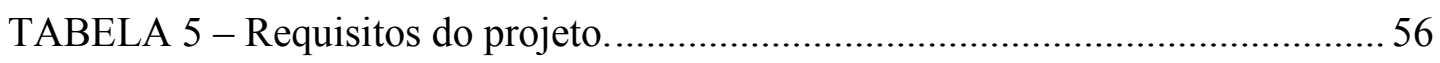

TABELA 6 - Parâmetros físicos do acoplador direcional........................................5 57

TABELA 7 - Parâmetros para a análise numérica do guia rib magnetoóptico ..........65 65

TABELA 8 - Parâmetros usados na simulação de condensados ópticos ................... 70

TABELA 9 - Relação entre as componentes minoritárias e a majoritária para incidência a $2^{\circ}$ na interface com o ar (v. Fig. 27 a Fig. 29).............................. 75

TABELA 10 - Relação entre as componentes minoritárias e a majoritária para incidência a $3^{\circ}$ na interface com o ar (v. Fig. 30 a Fig. 32) .............................. 76

TABELA 11 - Relação entre as componentes minoritárias e a majoritária para incidência a $4^{\mathrm{o}}$ na interface com o ar (v. Fig. 33 a Fig. 35).............................. 76

TABELA 12 - Relação entre as componentes minoritárias e a majoritária para colisão entre condensados ópticos em fase (v. Fig. 36 a Fig. 38) .88

TABELA 13 - Relação entre as componentes minoritárias e a majoritária para colisão entre condensados ópticos defasados de $180^{\circ}$ (v. Fig. 39 a Fig. 41)

TABELA 14 - Relação entre as componentes minoritárias e a majoritária para a incidência de condensado óptico em fenda de 1,0 $\mu \mathrm{m}$ de abertura (v. Fig. 43 a Fig. 45)

TABELA 15 - Relação entre as componentes minoritárias e a majoritária para a incidência de condensado óptico em fenda de 2,5 $\mu \mathrm{m}$ de abertura (v. Fig. 46 a Fig. 48) 
TABELA 16 - Relação entre as componentes minoritárias e a majoritária para a incidência de condensado óptico em fenda de $5 \mu \mathrm{m}$ de abertura (v. Fig. 49 a Fig. 51) 


\section{LISTA DE ABREVIATURAS E SIGLAS}

$\begin{array}{ll}\text { ADI } & - \text { Alternating Direction Implicit } \\ \text { CN } & - \text { Crank-Nicolson } \\ \text { DFT } & - \text { Discrete Fourier Transform } \\ \text { FD-BPM } & - \text { Finite Difference Beam Propagation Method } \\ \text { FDTD } & - \text { Finite Difference Time Domain } \\ \text { FFT } & - \text { Fast Fourier Transform } \\ \text { GD } & - \text { Generalized Douglas } \\ \text { GMIPML } & - \text { Generalized Material Independent Perfectly Matched Layer } \\ \text { PML } & - \text { Perfectly Matched Layer } \\ \text { SVEA } & - \text { Slow Varying envelope approximation } \\ \text { TBC } & - \text { Transparent Boundary Conditions } \\ \text { TE } & - \text { Transversal Electric } \\ \text { TM } & - \text { Transversal Magnetic }\end{array}$




\section{LISTA DE SÍMBOLOS}

\begin{tabular}{|c|c|}
\hline $\bar{B}$ & - Vetor densidade de fluxo magnético (weber/metro ${ }^{2}$ ) \\
\hline$c_{0}$ & - Velocidade da luz no vácuo $\left(2,99792458 \times 10^{8}\right.$ metro/segundo $)$ \\
\hline $\bar{D}$ & - Vetor densidade de fluxo elétrico $\left(\right.$ coulomb/metro $\left.{ }^{2}\right)$ \\
\hline $\bar{E}$ & - Vetor campo elétrico (volt/metro) \\
\hline $\bar{H}$ & - Vetor campo magnético (ampère/metro) \\
\hline$k_{0}$ & - Constante de propagação no vácuo (radiano/segundo) \\
\hline$n$ & - Índice de refração do meio (adimensional) \\
\hline neff & - Índice efetivo do modo guiado, $\beta / k_{0}$ (adimensional) \\
\hline$\alpha$ & - Coeficiente de não-linearidade $\left(\right.$ metro $^{2} /$ volt $\left.^{2}\right)$ \\
\hline$\beta$ & - Constante de fase do modo guiado (radiano/segundo) \\
\hline$\gamma$ & - Parâmetro função do mecanismo de não-linearidade (adimensional) \\
\hline$\delta$ & - Constante magnetoóptica (adimensional) \\
\hline$\delta t$ & - Passo de discretização temporal (segundo) \\
\hline$\delta x$ ou $\delta y$ & - Passo de discretização espacial transversal (metro) \\
\hline$\delta z$ & - Passo de discretização espacial longitudinal (metro) \\
\hline$\varepsilon_{0}$ & - Permissividade elétrica do vácuo $\left(8,854187 \times 10^{-12}\right.$ farad/metro $)$ \\
\hline$\lambda$ & - Comprimento de onda no vácuo (metro) \\
\hline$\mu$ & - Permeabilidade magnética do vácuo $\left(4 \pi \times 10^{-7}\right.$ henry/metro $)$ \\
\hline
\end{tabular}




\section{RESUMO}

Este trabalho tem por objetivo a extensão de métodos numéricos baseados em diferenças finitas no domínio do tempo (FDTD) e no domínio da freqüência (FDBPM) para a simulação da propagação de ondas eletromagnéticas em materiais com propriedades ópticas diversas, por exemplo, isotrópicos, anisotrópicos, lineares, nãolineares, bem como a combinação destes em uma mesma estrutura.

Inicialmente foram elaborados formalismos bidimensionais (FDTD e FD-BPM), dos quais foram investigados modos com polarização TM (Magnético Transversal) que se propagam em estruturas planares magnetoópticas/não-lineares/lineares. Esta polarização foi escolhida tendo em vista o campo magnetostático dc adotado, o qual possibilitou a observação do fenômeno não-recíproco associado ao não-linear simultaneamente.

Por outro lado, é bem sabido que o método FDTD é computacionalmente muito intensivo. Portanto, um grande esforço foi dedicado aos formalismos no domínio da freqüência, os quais foram implementados em duas e três dimensões. Este último foi estendido para um formalismo totalmente vetorial, capaz de simular modos híbridos ou até mesmo a transferência de energia entre modos de polarizações ortogonais. Isto nos permitiu investigar geometrias ainda mais complexas, tais como um isolador óptico baseado em um guia de onda tip rib utilizando material magnetooptico. Adicionalmente, fenômenos de natureza complexa, tais como a dinâmica dos condensados de luz em materiais com não-lineares do tipo Kerr com saturação, também conhecidos como meios não-lineares cúbico-qüínticos, foram investigados pela primeira vez com um formalismo vetorial.

Finalmente, métodos numéricos capazes de considerar qualquer combinação de materiais com propriedades ópticas distintas (linear e/ou não-linear e/ou magnetoóptico) são uma ferramenta extraordinária para a comunidade científica para 
o projeto de novos dispositivos ópticos, bem como a investigação de novos efeitos físicos com vistas à aplicações em computação óptica, que podem resultar em um menor e mais eficiente número de componentes para sistemas de comunicações ópticos. 


\begin{abstract}
This work introduces three improved formalisms for the analysis of electromagnetic wave propagation through materials with distinct optical properties, i.e., isotropic, anisotropic, linear, nonlinear, or any combination of them. Two finite difference approaches were extensively investigated in this work for this purpose, namely the finite difference in time domain (FDTD), and the finite difference beam propagation method (2D and 3D FD-BPM), these in frequency domain.

Initially, a TM (transverse magnetic) mode propagating through a planar magnetooptic/nonlinear/linear waveguide was investigated by way of a twodimensional formalism (FDTD and FD-BPM). This mode polarization was chosen based on the orientation of the external magnetostatic field adopted, which favored the observation of non-reciprocal and nonlinear effects simultaneously.

On the other hand, it is well known that FDTD formalisms are computationally intensives. Therefore, a great effort was dedicated to its frequency domain counterpart (FD-BPM), which was implemented in two and three dimensions. The later was further extended to a fully vectorial formalism, which is capable of simulating hybrid modes or even the energy transfer between orthogonal modes. This enabled us to investigate more complex geometries, such as an optical isolator based on magnetooptic rib waveguide. Additionally, complex phenomena, such as the dynamic of light condensates in bulk nonlinear Kerr media with saturation, also known as cubic-quintic nonlinear media, were investigated for the first time with a $3 \mathrm{D}$ vectorial formalism.

Finally, numerical methods capable of handling any combination of materials with distinct optical properties (linear and/or nonlinear and/or magnetooptic) are an extraordinary tool for the scientific community for the design of new optical devices, as well as the investigation of new physical effects aimed for optical computing, that
\end{abstract}


may result in fewer and more efficient components for optical communication systems. 


\section{INTRODUÇÃO}

A crescente demanda por um maior volume de informações transportado pelos sistemas de comunicações atuais vem exigindo o desenvolvimento de componentes ópticos cada vez mais sofisticados. Esse avanço passa, necessariamente, pelo projeto de dispositivos ópticos tais como: lasers, moduladores, isoladores ópticos, filtros, chaves ópticas, etc. Em sua grande maioria, esses dispositivos estão disponíveis individualmente. A utilização desses dispositivos ao longo de um enlace de comunicações pode, muitas vezes, introduzir uma perda de inserção considerável. Portanto, o projeto de dispositivos ópticos que incorporem funções antes desempenhadas pela associação de dois ou mais desses dispositivos permitirá um considerável aumento na eficiência de sistemas de comunicações ópticos, com uma conseqüente redução nos custos de implantação e manutenção dos mesmos. Porém, o fator limitante para o projeto de tais dispositivos consiste na disponibilidade de modelos matemáticos confiáveis, ou seja, modelos que não façam uso de aproximações que venham a limitar seu grau de aplicabilidade. Isto acontece geralmente em modelos analíticos. Neste caso, métodos numéricos passam a ser a opção natural para a simulação de estruturas mais complexas.

\subsection{O método das diferenças finitas no domínio do tempo (FDTD)}

O método numérico FDTD vem sendo progressivamente adotado para a solução de problemas gerais em eletromagnetismo. No entanto, este método apresenta a característica de requerer a realização de uma quantidade considerável de operações numéricas, o que vem a ser um fator que dificulta a sua aplicação em problemas de configurações mais complexas ou detalhadas. Contudo, esta "limitação" vem se tornando cada vez mais passível de ser superada por causa do aumento progressivo 
na capacidade de processamento dos computadores a cada ano associado ao desenvolvimento de técnicas de processamento paralelo. Esta evolução tem propiciado que métodos no domínio do tempo, sejam em diferenças finitas ou em elementos finitos, passem a ser a escolha ideal para a modelagem destas novas estruturas.

Ao contrário de outras técnicas numéricas, nas quais são aplicadas várias aproximações (como a paraxial, por exemplo) restringindo a solução de problemas a certos casos, o método FDTD é bastante abrangente e consiste de uma solução de onda completa para os campos eletromagnéticos obtida diretamente das equações de Maxwell no domínio do tempo. O passo de discretização adotado deve ser consideravelmente menor que um comprimento de onda, o que implica na utilização de dezenas de Mbytes e dezenas de milhares de passos de tempo na evolução dos campos para resolver alguns problemas práticos [1]. Contudo, os resultados geralmente justificam a demora de processamento pelo fato de que o número de aproximações é virtualmente eliminado. A precisão com que os campos eletromagnéticos são obtidos é determinada pela resolução do domínio espacial numérico e passo temporal. Além do mais, para muitos dos dispositivos ópticos compactos atuais, os quais se estendem por apenas alguns comprimentos de onda, a quantidade de processamento requerida pelo método FDTD não é proibitiva [1].

O emprego do método FDTD na análise de guia de ondas combinando meios não-lineares e não-recíprocos ainda não havia sido explorado na literatura. Em relação a meios anisotrópicos, o esquema FDTD original de Yee [2] pode ser utilizado desde que os tensores sejam diagonalizáveis. Esta característica pode ser observada no espalhador discreto apresentando um tensor permissividade anisotrópico, porém diagonal, sugerido por Taflove [3] em 1990. Em 1993, Schneider e Hudson [4] propuseram uma extensão do método FDTD para incluir tensores não-diagonais, a qual baseia-se em interpolações utilizando valores de componentes de campo em células vizinhas.

O método FDTD foi aplicado pela primeira vez na modelagem da propagação de ondas em meios não-lineares por Merewether e Radasky [5], que analisaram uma cavidade cilíndrica parcialmente preenchida na qual a condutividade é uma função do campo elétrico. O método FDTD também foi empregado no estudo da propagação 
através de um material magnético não-linear altamente condutor por Luebbers et al. [6]. Neste caso, a permeabilidade do material, por imposição, era modificada a cada passo de tempo. O efeito da alta condutividade e da não-linearidade do material magnético sobre a estabilidade da formulação FDTD também foi considerado nesta análise.

Jamid e Albader [7], por sua vez, propuseram uma técnica relativamente fácil visando a utilização do método FDTD na solução de problemas envolvendo guias de ondas ópticos contendo meios não-lineares. Alternativamente, Ziolkowski e Judkins [8]-[11] apresentaram um modelo caracterizado pela discretização de quantidades como a corrente de polarização e o vetor polarização, além dos campos elétrico e magnético. Reinex et al. [12] introduziram um modelo não-linear e dispersivo para a análise da propagação de sólitons. Como exemplo da aplicação do método FDTD no auxílio de projeto de dispositivos, pode ser destacado o trabalho de Shiozawa e Yoshitake [13], no qual é investigada a melhoria da eficiência na conversão de energia para um laser Cherenkov contendo um meio do tipo Kerr. Sulivan [14],[15] propôs uma formulação FDTD baseada em transformadas $Z$ que pode ser usada na modelagem de material dispersivo não-linear. Uma metodologia FDTD alternativa para não-linearidades do tipo Kerr foi proposta por Tran [16], na qual o campo elétrico é obtido a partir da densidade de fluxo por intermédio da solução de uma equação cúbica.

A fim de evitar erros de análise provocados por reflexões indesejadas nos limites espaciais numéricos de volta à janela computacional, devem ser adotadas condições de contorno absorventes (Absorbing Boundary Conditions ou ABC) eficientes, de modo que tais reflexões sejam anuladas sem elevar a complexidade do tratamento numérico dos campos no domínio espacial em análise. Várias técnicas de absorção foram propostas na literatura, podendo ser divididas em duas classes: 1) condições de contorno absorventes baseadas em equação diferencial e 2) condições de contorno absorventes baseadas em material. Em geral, as ABC's baseadas em equação diferencial são obtidas pela fatoração da equação de onda e pela utilização de uma solução que permita apenas ondas que propaguem para fora do domínio computacional [17], tais como as técnicas propostas por Mur [18] e Higdon [19][20]. 
As ABC's baseadas em material utilizam camada adicional de células contornando o domínio computacional em questão. Esta camada é constituída de um meio especial na qual os campos são atenuados à medida que propagam no mesmo. Originalmente, as ABC's baseadas em material não proporcionavam um índice suficientemente baixo de reflexões nos contornos devido ao fato de que o casamento da impedância característica do meio absorvente com a impedância característica do espaço livre ocorra apenas no caso de incidência normal [17]. A introdução da parede perfeitamente casada (Perfect Matched Layer ou PML) por Berenger [21], foi um marco no avanço desta classe de ABC's, por mostrar uma maior eficiência na redução das ondas refletidas nos limites em relação a qualquer outra $\mathrm{ABC}$ proposta anteriormente. A camada absorvente de Berenger se constitui num meio fictício por apresentar uma condutividade magnética $\left(\sigma^{*}\right)$, além da condutividade elétrica $(\sigma)$, das quais são estimadas condições para o perfeito casamento entre a camada absorvente e o espaço livre para qualquer ângulo de incidência. A desvantagem desta técnica, em seu formato original, é a necessidade da decomposição de componentes de campo para o controle da atenuação em função da direção de propagação, elevando a complexidade do tratamento numérico dos campos eletromagnéticos, o tempo de processamento e a utilização de memória principal, o que motivou a realização de novos trabalhos englobando a análise crítica da técnica e a proposição de versões modificadas da mesma, como em [22]-[24]. Uma versão modificada do formalismo original da PML foi proposta por Sullivan [25], com aplicação da idéia em materiais especiais investigada por Zhao [26]. O formalismo proposto atua na absorção direta dos vetores densidade de fluxo elétrico e magnético e é, portanto, independente das relações constitutivas do material. Tais relações constitutivas são empregadas no cálculo posterior dos vetores campo elétrico e magnético, os quais são indiretamente absorvidos na região desta PML independente de material.

Apesar da crescente popularização do método numérico FDTD devido à sua vasta aplicabilidade, a simulação de problemas bidimensionais mais amplos ou tridimensionais ainda requer a adoção de sistemas computacionais de última geração, aumentando proibitivamente os custos de pesquisa. Portanto, muitas vezes se torna conveniente verificar a possibilidade de prover paralelamente uma outra ferramenta que resolva um considerável conjunto de problemas de ondas eletromagnéticas, 
menos flexível mas com uma maior rapidez. Um exemplo de tal ferramenta é o método da propagação de feixe, BPM.

\section{2 método da propagação de feixe (BPM)}

O método da propagação de feixe bidimensional, em sua forma paraxial [27], é uma das ferramentas mais eficazes para a análise de dispositivos ópticos baseados em guias planares tais como: moduladores, junções $\mathrm{Y}$, chaves ópticas, etc. Contudo, este método apresenta sérias limitações em aplicações nas quais a propagação do feixe ocorre em ângulos elevados em relação à direção longitudinal. Para amenizar este problema foram propostas formulações FD-BPM de ângulo largo utilizando aproximantes de Padé [28]. As condições de contorno utilizadas para evitar reflexões nos limites numéricos pode ser a PML [29]-[31] ou a condição de contorno transparente (TBC) [32].

Até recentemente, trabalhos a respeito do BPM eram predominantemente dirigidos a guias ópticos isotrópicos convencionais. A consideração de materiais especiais consistiu em uma evolução necessária para a expansão da aplicabilidade do método a problemas contemporâneos. O primeiro BPM desenvolvido para meios anisotrópicos foi proposto por Thilen e Yevick [33], o qual era baseado na obsoleta versão FFT (Fast Fourier Transform) do método. Tsuji et al. [34] desenvolveram uma formulação de ângulo largo para meios anisotrópicos baseada em elementos finitos (FE-BPM). Mais recentemente, a implementação de um FD-BPM de ângulo largo também para materiais anisotrópicos foi realizada por Gonçalves [35].

Em se tratando da aplicação do BPM a estruturas não-lineares, HérnandezFigueroa [36] introduziu um método iterativo aplicado ao FE-BPM com o objetivo de evitar erros de convergência do campo na região não-linear. Yokota et al. [37] desenvolveram um formalismo baseado em diferenças finitas ao qual é aplicada técnica iterativa similar. Para diminuir a sensibilidade do erro dos resultados obtidos com o ângulo de propagação, inerente do BPM paraxial, estruturas não-lineares foram analisadas com o FE-BPM de ângulo largo proposto por Yasui et al., [38] a qual era baseada em aproximantes de Padé. Uma investigação mais detalhada sobre a aplicação de aproximantes de Padé ao FD-BPM para meios não-lineares foi 
realizada por Flamino [39], o qual propôs técnicas otimizadas para implementações de ângulo largo baseadas em aproximantes de ordens diversas.

Portanto, uma vez dominados os formalismos para simulação de guias de ondas baseados em meios não-lineares ou meios não-recíprocos, individualmente, surgiu em 1994 a idéia de se combinar estes materiais de forma a produzir um dispositivo apresentando um funcionamento híbrido, ou seja, de chave e isolador óptico simultaneamente. Esta proposta foi elaborada por Gniadek [40], que desenvolveu a relação de dispersão para um guia não-linear e magnetoóptico de três camadas, analisando a partir desta relação as propriedades de isolamento e chaveamento óptico da estrutura em função da densidade de potência óptica incidente.

Em meios magnetoópticos, a anisotropia é induzida pela presença de um campo magnetostático $\left(\mathrm{H}_{\mathrm{DC}}\right)$, o qual, quando devidamente orientado, torna possível separar as soluções das equações de Maxwell em modos TE e TM. Assim, o comportamento não-recíproco é baseado na diferença entre as constante de propagação no sentido direto $(+z)$ e reverso $(-z)$ para os modos TM. Paralelamente, devido à presença de duas componentes de campo elétrico, a análise de modos TM em meios não-lineares se torna mais complexa em relação à de modos TE. A distinção entre os mecanismos de não-linearidade, tais como distorção eletrônica, orientação molecular, efeitos térmicos ou eletrostrição, não vinha sendo considerada de forma ampla na literatura, que usualmente limitava-se à análise do mecanismo que proporciona o formalismo mais simples.

Formalismos tridimensionais, por sua vez, são atraentes para a modelagem de dispositivos ópticos que apresentam uma geometria mais arbitrária, tais como fibras ópticas ou guias do tipo rib, por exemplo. Para a análise de tais estruturas, o método de propagação de feixe tridimensional (BPM-3D) é uma opção eficiente, pois a propagação em guias muito longos em relação ao comprimento de onda pode ser simulada em computadores convencionais num tempo razoável, enquanto que o método FDTD-3D exigiria a utilização de supercomputadores ou clusters de processamento paralelo, devido à quantidade consideravelmente maior de processamento e de memória necessários.

A implementação de métodos BPM-3D vetoriais é substancialmente mais atraente quando comparados às versões escalar e semi-vetorial [41], pois isto permite 
uma análise mais precisa de estruturas apresentando geometria arbitrária ou materiais especiais, casos nos quais podem ocorrer a propagação de modos híbridos ou a transferência de potência entre modos ortogonalmente polarizados. Métodos vetoriais são freqüentemente implementados usando discretizações baseadas em diferenças finitas (FD) ou elementos finitos (FE). Em ambos os casos, a equação de Helmholtz vetorial é discretizada de modo que uma relação recursiva entre as distribuições transversais de campo no intervalo de um passo longitudinal seja obtida.

O formalismo vetorial descrito for Xu et al. [42] é aplicável apenas para guias ópticos com baixa anisotropia e fraco efeito geométrico sobre a polarização. Tsuji et al. [43] propuseram um algoritmo BPM escalar tridimensional (3D) baseado em elementos finitos que considera a propagação óptica apenas em estruturas quasiplanares ou fracamente guiantes. Uma análise mais completa foi realizada por Sellere et al. [44], pela consideração de tensores cheios para representar tanto a permissividade dielétrica quanto a permeabilidade magnética em um formalismo baseado em elementos finitos.

Uma das técnicas numericamente mais eficientes para a solução de problemas tridimensionais é o método implícito de direções alternantes (ADI). O método ADI é baseado em uma técnica de divisão de operador, o que torna possível reduzir o tamanho do problema utilizando um esquema que é parte implícito e parte iterativo. Cada passo de propagação é dividido em dois subpassos e, em cada subpasso, uma dimensão é tratada implicitamente. A Técnica ADI tem sido empregada com sucesso na solução problemas tridimensionais escalares e semivetoriais, conforme os trabalhos de Yamauchi et al. e Liu et al. [45]-[46], respectivamente. Em ambos os casos, uma divisão do tipo Peaceman-Rachford foi aplicada. Em relação a formalismos BPM completamente vetoriais, entretanto, uma técnica ADI mais elaborada se torna necessária [47]-[48]. Algoritmos BPM para meios anisotrópicos ou não-lineares que exploram as vantagens da técnica ADI ainda não haviam sido propostos na literatura.

\subsection{Contribuições desta tese}


O primeiro êxito atingido durante este trabalho foi a aplicação do método FDTD na investigação dos efeitos de não-linearidade e não-reciprocidade, simultaneamente, em um guia planar.

Em seguida, a implementação de um método de propagação de feixe (FD-BPM) bidimensional expandido para a consideração de meios não-lineares e anisotrópicos possibilitou a validação dos resultados obtidos previamente pelo FDTD, além da simulação de seções mais extensas de guias de ondas. Este método consiste em contribuição inédita em termos de formalismos numéricos, no qual buscou-se minimizar ao máximo as aproximações comumente empregadas no tratamento numérico da equação de Helmholtz para análise de modos TM em meios nãolineares do Tipo Kerr. No caso, o método apresenta formalismo de ângulo largo, inclui todas as derivadas longitudinais de permissividade dielétrica e considera qualquer mecanismo de não-linearidade. Por meio deste método, foi proposto um novo dispositivo capaz de combinar as funções de isolador e chave óptica, o qual é baseado num acoplador direcional não-linear e não-recíproco.

Outra contribuição importante é a elaboração do método FD-BPM tridimensional vetorial para meios não-lineares ou que apresentem anisotropia transversal. O método proposto combina pela primeira vez as técnicas implícita de direções alternantes (ADI) e leapfrog para a simulação eficiente da propagação óptica em estruturas tridimensionais. Nesta tese, o principal destaque da aplicação deste método está na simulação da propagação de condensados ópticos em uma abordagem vetorial. Estes condensados são formados em um meio não-linear do tipo Kerr com saturação.

\subsection{Publicações}

As publicações ocorridas durante o período do trabalho são:

1. L. D. Alcantara, F. L. Teixeira, A.C. César, e B.V. Borges, "FDTD analysis of nonlinear and nonreciprocal integrated optical waveguides", 2001 SBMO/IEEE MTT-S International Microwave and Optoelectronics Conference - IMOC, August 6-10, 2001, Belém-PA, Brazil. 
2. João M.D. Ferreira, Licinius D. Sá de Alcantara, Ben-Hur V. Borges e Amílcar C. César, “Análise de Filtro Óptico Ressonante pelo Método das Diferenças Finitas no Domínio do Tempo Bidimensional”, $19^{\circ}$ Simpósio Brasileiro de Telecomunicações-SBrT 2001, Fortaleza, 3 a 6 de setembro de 2001, CE.

3. L. D. Alcantara, F. L. Teixeira, A.C. César, e B.V. Borges, "A novel wide-angle FD-BPM for nonlinear and nonreciprocal waveguides", 2002 IEEE Conference on Electromagnetic Field Computation - CEFC, June 16-19, 2002, Perugia, Italy.

4. L. D. Alcantara, F. L. Teixeira, A C. Cesar e B. V. Borges, "Wide-Angle FDBPM Analysis of TM Modes in Nonlinear and Nonreciprocal Integrated Optical Waveguides", 2002 Progress in Electromagnetics Research-PIERS, Cambridge, June 1-5, 2002.

5. Licinius D. S. Alcantara, Fernando L. Teixeira, Amilcar C. Cesar e Ben-Hur V. Borges, "Projeto de um Dispositivo Híbrido Chave-Isolador Óptico Utilizando o Método FD-BPM de Ângulo Largo", X Simpósio Brasileiro de MicroondasSBMO, Recife, 12 a 16 de agosto de 2002.

6. Licinius D. S. Alcantara, Fernando L. Teixeira, Amilcar C. Cesar e Ben-Hur V. Borges, “An Improved Wide-Angle FD-BPM for Nonlinear and Nonreciprocal Waveguides", IEEE Transactions on Magnetics, vol. 39, no. 3, pp. 1223-1226, May 2003.

7. Reinaldo S. Flamino, Licinius D. S. Alcantara, Amílcar C. César e Ben-Hur V. Borges, "Wide-Angle Solution of Nonlinear Coupled Wave Equations Based on Padé Approximants", Microwave and Optical Technology Letters, vol 38, no. 2., pp. 108-113, Jul 2003.

8. Licinius D. S. Alcantara, Fernando L. Teixeira, Amilcar C. Cesar e Ben-Hur V. Borges, "Full-Vector Finite-Difference Beam Propagation Method for the Analysis of Three-Dimensional Anisotropic Optical Waveguides", International Microwave and Optoelectronics Conference - IMOC'2003, Foz do Iguaçu, PR, 20 a 23 de Setembro de 2003.

9. Licinius D.S. Alcantara, Marcos A.C. Lima, Aluízio F.R. Araújo, Amilcar C. César e Ben-Hur V. Borges, "Projeto de Isolador Óptico Utilizando Algoritmo 
Genético", $20^{\circ}$ Simpósio Brasileiro de telecomunicações - SBrT’2003, Rio de Janeiro, RJ, 05-08 de Outubro de 2003.

\subsection{Organização do texto}

O texto está disposto da seguinte forma: o Capítulo 2 descreve a expansão do método FDTD, no qual a formulação geral será particularizada para a propagação de modos TM em estruturas bidimensionais contendo materiais anisotrópicos e nãolineares. O método FD-BPM bidimensional é descrito no Capítulo 3. No Capítulo 4, um formalismo FD-BPM vetorial tridimensional, baseado na técnica ADI, é proposto para meios não-lineares e anisotrópicos. No Capítulo 5 são expostos problemas bidimensionais, com resultados obtidos utilizando o método FDTD e/ou FD-BPM descritos nos Capítulos 2 e 3, respectivamente. O Capítulo 6 expõe alguns problemas tridimensionais envolvendo materiais especiais, com resultados obtidos por intermédio do método FD-BPM descrito no Capítulo 4. No Capítulo 7 são expostas as conclusões gerais e finais. 


\section{O MÉTODO FDTD}

Este capítulo tem como objetivo proporcionar uma visão geral da aplicação do método FDTD na análise de problemas eletromagnéticos, os quais podem englobar diversos tipos de meios, tais como isotrópicos, anisotrópicos e não-lineares.

\subsection{Discretização temporal das equações de Maxwell}

As equações de Maxwell em sua forma rotacional, para meios livres de cargas, são dadas por

$$
\begin{aligned}
& \frac{\partial \vec{B}}{\partial t}=-\nabla \times \vec{E}, \\
& \frac{\partial \vec{D}}{\partial t}=\nabla \times \vec{H},
\end{aligned}
$$

nas quais $\vec{B}$ e $\vec{D}$ são os vetores densidade de fluxo magnético e elétrico, respectivamente. $\vec{H}$ e $\vec{E}$ são os vetores campo magnético e elétrico, respectivamente. As leis de Gauss para as densidades de fluxo elétrico e magnético não precisam ser empregadas no formalismo FDTD tradicional, pois estas são teoricamente uma conseqüência direta das equações rotacionais de Maxwell. Contudo, o domínio espacial numérico deve ser estruturado de forma que as leis de Gauss sejam implícitas nas posições das componentes dos vetores campo elétrico e magnético na malha e das operações de derivadas espaciais numéricas sobre essas componentes que modelam a ação do operador rotacional [54].

Considerando que os meios envolvidos podem apresentar propriedades distintas e/ou complexas, as relações constitutivas não serão explicitadas nesta seção. Isto será feito na Seção 2.6, onde um problema particular é proposto. 
Discretizando (2.1) e (2.2) no tempo e considerando o papel das relações constitutivas no complemento do processo recursivo, obtém-se

$$
\begin{aligned}
\vec{B}^{n+\frac{1}{2}} & =\vec{B}^{n-\frac{1}{2}}-\delta t \nabla \times \vec{E}^{n}, \quad \vec{B}^{n+\frac{1}{2}} \underset{\text { constitutiva }}{\stackrel{\text { relação }}{\Longrightarrow} \vec{H}^{n+\frac{1}{2}}} \\
\vec{D}^{n+1} & =\vec{D}^{n}+\delta t \nabla \times \vec{H}^{n+\frac{1}{2}}, \quad \vec{D}^{n+1} \underset{\text { constitutiva }}{\Longrightarrow} \vec{E}^{n+1}
\end{aligned}
$$

Portanto, os campos desejados $\vec{H}^{n+\frac{1}{2}}$ e $\vec{E}^{n+1}$ são determinados indiretamente por meio das relações constitutivas, cujas expressões podem variar de um material para outro.

\subsection{Discretização espacial das equações de Maxwell}

Esta etapa consiste na discretização das derivadas espaciais contidas nos rotacionais dos campos elétrico e magnético em (2.3) e (2.4), o que depende da configuração da célula utilizada. Nesta seção, são abordadas a célula original de Yee, a qual foi empregada nas simulações (FDTD) deste trabalho, e a célula de dois nós (two-node cell), esta apenas a título de exposição. Esta seção expõe a versão tridimensional de cada configuração, a partir da qual a redução para o caso bidimensional ou unidimensional é direta. Isto pode ser feito fazendo-se a largura da célula ao longo do eixo $x, y$ ou $z$ tender a zero.

\subsubsection{Discretização espacial usando célula de Yee}

Esta configuração é parte intrínseca da idealização do método [49], sendo a mais utilizada tradicionalmente (Fig. 1). A disposição intercalada das componentes de campo na malha resultante proporciona a redução de erros numéricos na simulação de ondas eletromagnéticas que se propagam em meios isotrópicos. Porém, esta configuração pode apresentar limitações na caracterização da propagação em meios mais complexos, em casos nos quais são requeridos valores de componentes em posições nas quais estas não existem. Visando contornar este problema e estender a aplicação desta configuração a meios anisotrópicos com tensores não diagonalizáveis, Schneider e Hudson [4] propuseram a aproximação de valores de 
componentes em certas posições utilizando seus valores conhecidos (de suas posições reais) em células adjacentes.

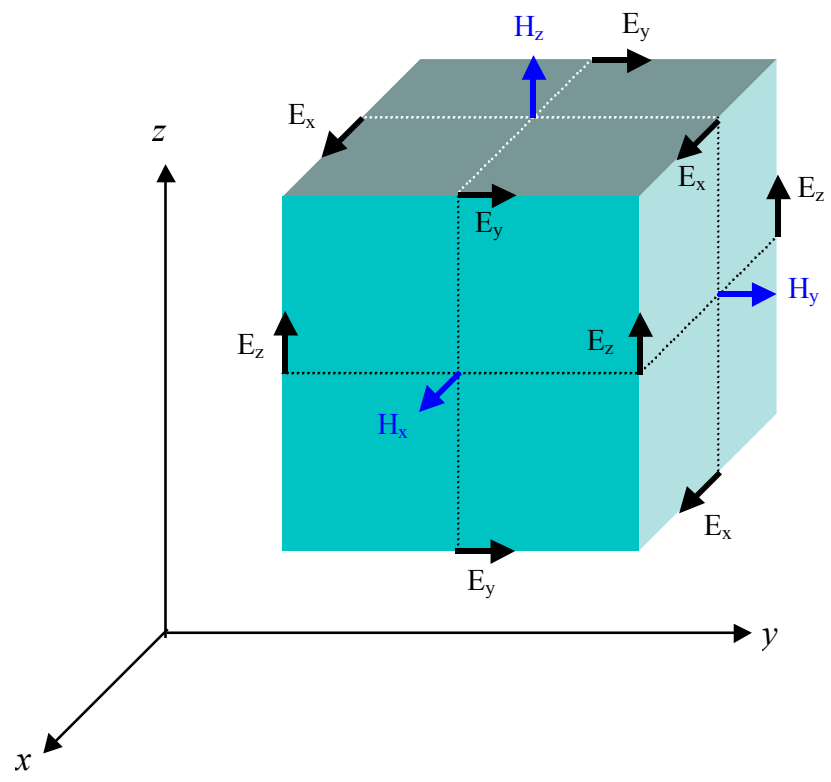

Fig. 1 - Célula de Yee tridimensional

A discretização dos rotacionais baseando-se na célula da Fig. 1, fornece:

$$
\begin{aligned}
& \nabla \times \bar{E} \approx\left(\begin{array}{l}
\frac{1}{\delta y}\left[E_{z\left(I, J+1, K+\frac{1}{2}\right)}-E_{z\left(I, J, K+\frac{1}{2}\right)}\right]-\frac{1}{\delta z}\left[E_{y\left(I, J+\frac{1}{2}, K+1\right)}-E_{y\left(I, J+\frac{1}{2}, K\right)}\right] \\
\frac{1}{\delta z}\left[E_{x\left(I+\frac{1}{2}, J, K+1\right)}-E_{x\left(I+\frac{1}{2}, J, K\right)}\right]-\frac{1}{\delta x}\left[E_{z\left(I+1, J, K+\frac{1}{2}\right)}-E_{z\left(I, J, K+\frac{1}{2}\right)}\right] \\
\frac{1}{\delta x}\left[E_{y\left(I+1, J+\frac{1}{2}, K\right)}-E_{y\left(I, J+\frac{1}{2}, K\right)}\right]-\frac{1}{\delta y}\left[E_{x\left(I+\frac{1}{2}, J+1, K\right)}-E_{x\left(I+\frac{1}{2}, J, K\right)}\right]
\end{array}\right) \\
& \nabla \times \bar{H} \approx\left(\begin{array}{l}
\frac{1}{\delta y}\left[H_{z\left(I+\frac{1}{2}, J+\frac{1}{2}, K\right)}-H_{z\left(I+\frac{1}{2}, J-\frac{1}{2}, K\right)}\right]-\frac{1}{\delta z}\left[H_{y\left(I+\frac{1}{2}, J, K+\frac{1}{2}\right)}-H_{y\left(I+\frac{1}{2}, J, K-\frac{1}{2}\right)}\right] \\
\frac{1}{\delta z}\left[H_{x\left(I, J+\frac{1}{2}, K+\frac{1}{2}\right)}-H_{x\left(I I J+\frac{1}{2}, K-\frac{1}{2}\right)}\right]-\frac{1}{\delta x}\left[H_{z\left(I+\frac{1}{2}, J+\frac{1}{2}, K\right)}-H_{z\left(I-\frac{1}{2}, J+\frac{1}{2}, K\right)}\right] \\
\frac{1}{\delta x}\left[H_{y\left(I+\frac{1}{2}, J, K+\frac{1}{2}\right)}-H_{y\left(I-\frac{1}{2}, J, K+\frac{1}{2}\right)}\right]-\frac{1}{\delta y}\left[H_{x\left(I, J+\frac{1}{2}, K+\frac{1}{2}\right)}-H_{x\left(I, J-\frac{1}{2}, K+\frac{1}{2}\right)}\right)
\end{array}\right)
\end{aligned}
$$

\subsubsection{Discretização espacial usando célula de dois nós}

A configuração de dois nós (two-node), mostrada na Fig. 2, foi proposta como alternativa à célula de Yee por Bi et. al [50], com a vantagem de apresentar uma 
maior estabilidade. Esta configuração permite utilizar um incremento temporal superior ao limite que seria possível com o esquema de Yee, para uma determinada resolução de malha, sem a resposta divergir, porém resulta em um número quatro vezes maior de operações em cada passo temporal.

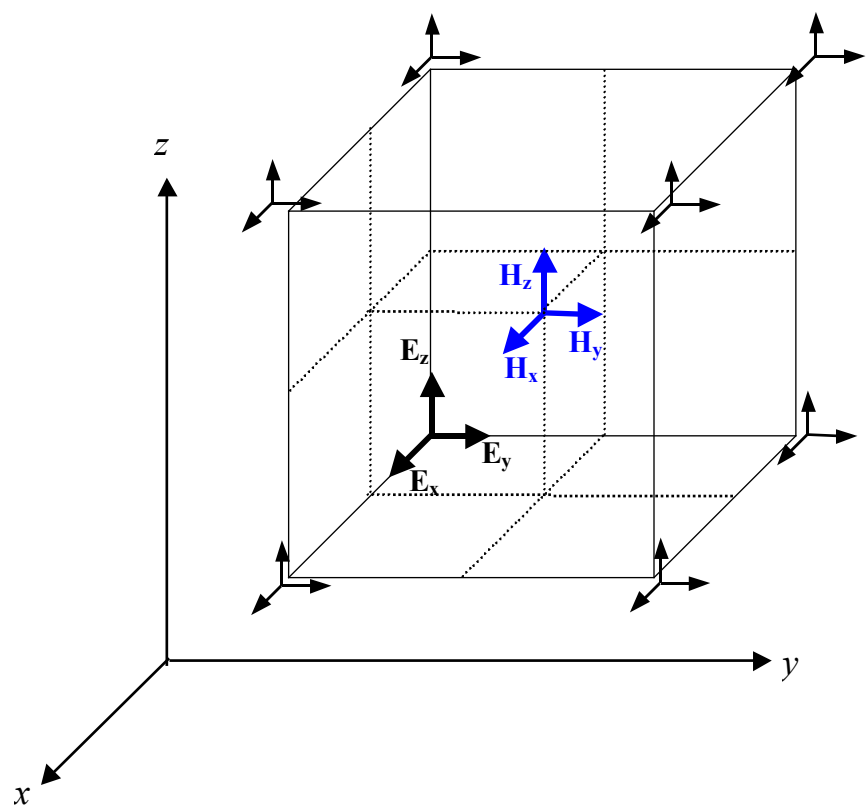

Fig. 2 - Célula de dois nós tridimensional

A discretização espacial consiste na aproximação da derivada da componente de campo ao longo de um eixo seguida de duas médias da mesma, sendo uma média ao longo de cada eixo restante. Assim, as derivadas espaciais das componentes de campo elétrico ficam centradas na posição das componentes de campo magnético e vice-versa. Note que a redução para o caso bidimensional também descarta a realização da média ao longo da dimensão eliminada. Para a célula da Fig. 2, a expressão discretizada dos rotacionais é dada por

$$
\begin{aligned}
& \nabla \times \bar{E} \approx \\
& \frac{1}{4}\left(\begin{array}{c}
\frac{1}{\delta y} \sum_{r=0}^{1} \sum_{p=0}^{1}\left[E_{z(I+p, J+1, K+r)}-E_{z(I+p, J, K+r)}\right]-\frac{1}{\delta z} \sum_{q=0}^{1} \sum_{p=0}^{1}\left[E_{y(I+p, J+q, K+1)}-E_{y(I+p, J+q, K)}\right] \\
\frac{1}{\delta z} \sum_{q=0}^{1} \sum_{p=0}^{1}\left[E_{x(I+p, J+q, K+1)}-E_{x(I+p, J+q, K)}\right]-\frac{1}{\delta x} \sum_{r=0}^{1} \sum_{q=0}^{1}\left[E_{z(I+1, J+q, K+r)}-E_{z(I, J+q, K+r)}\right] \\
\frac{1}{\delta x} \sum_{r=0}^{1} \sum_{q=0}^{1}\left[E_{y(I+1, J+q, K+r)}-E_{y(I, J+q, K+r)}\right]-\frac{1}{\delta y} \sum_{r=0}^{1} \sum_{p=0}^{1}\left[E_{x(I+p, J+1, K+r)}-E_{x(I+p, J, K+r)}\right]
\end{array}\right)
\end{aligned}
$$


$\nabla \times \bar{H} \approx$

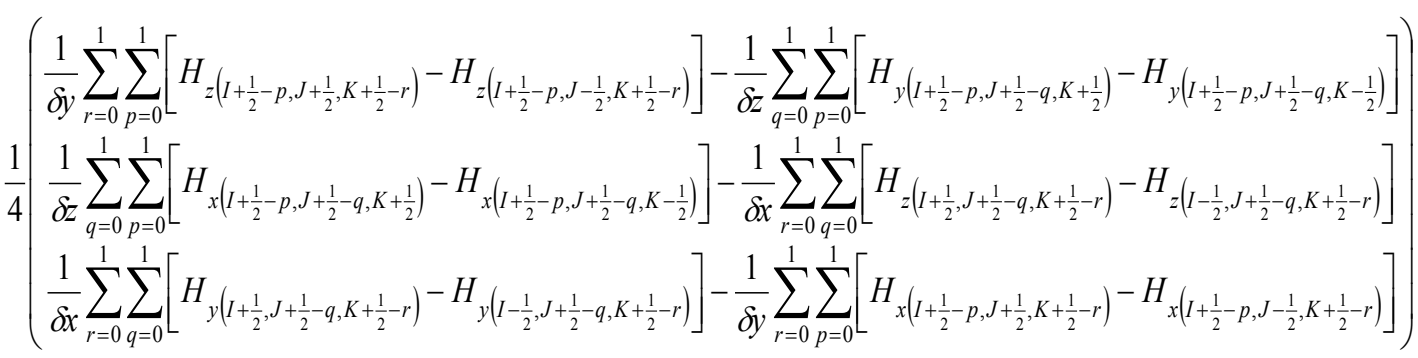

Devido ao fato das componentes de campo elétrico estarem situadas em um único ponto, assim como as de campo magnético, esta célula já é compatível com a caracterização da propagação em meios anisotrópicos genéricos [51]. Contudo, por ser um modelo ainda pouco explorado na literatura, algumas precauções devem ser tomadas, como por exemplo, acerca da possibilidade de surgimento de modos espúrios devido as médias realizadas nas derivadas espaciais, as quais poderiam afetar numericamente a lei de Gauss para o divergente das densidades de fluxo elétrico e de fluxo magnético.

A aplicação desta célula também pode ser determinante em situações nas quais há uma variação acentuada da distribuição de campo na região não-linear e, portanto, problemas de convergência no procedimento iterativo poderão ocorrer para a célula de Yee (uma conseqüência da configuração distribuída das componentes de campo).

\subsection{Critérios para precisão e estabilidade}

A precisão do método relaciona a qualidade dos resultados com a resolução da malha. Na prática, o comprimento da aresta das células deve corresponder a uma pequena fração do comprimento de onda (aproximadamente $1 / 10$ ou menor). Para a caracterização de um modelo estável é utilizado o critério de Courant [52], o qual impõe um limite ao passo temporal a ser escolhido, de acordo com a resolução da malha e a máxima velocidade de propagação na estrutura. Para um modelo de discretização de Yee em três, duas (plano $x z$ ) e uma dimensão (eixo $z$ ), respectivamente, o critério de estabilidade de Courant é dado por 


$$
\begin{aligned}
& \delta t \leq \frac{c_{0} \sqrt{\left(\frac{1}{\delta x}\right)^{2}+\left(\frac{1}{\delta y}\right)^{2}+\left(\frac{1}{\delta z}\right)^{2}}}{\delta t} \leq \frac{n_{\min }}{c_{0} \sqrt{\left(\frac{1}{\delta x}\right)^{2}+\left(\frac{1}{\delta z}\right)^{2}}} \\
& \delta t \leq \frac{\delta z \cdot n_{\min }}{c_{0}}
\end{aligned}
$$

nas quais $c_{0}$ é a velocidade da luz no vácuo e $n_{\min }$ é o menor índice de refração na estrutura.

\subsection{Transformada discreta de Fourier (DFT)}

A transformada discreta de Fourier (DFT) é uma ferramenta bastante útil para realizar conversões do domínio do tempo ou do espaço para o domínio da freqüência ou do espaço inverso, respectivamente. Isto aumenta quantitativa e qualitativamente a gama de resultados que o método FDTD pode fornecer, passando a ser um método bastante utilizado também para o cálculo de parâmetros no domínio da freqüência.

Por intermédio de técnicas de conversão, como a DFT, uma resposta espectral em uma banda de freqüências de interesse pode ser obtida numa única simulação usando um pulso de excitação banda larga. Furse e Gandhi [53] compararam a DFT com a transformada rápida de Fourier (FFT) e concluíram que a DFT aplicada ao FDTD é mais eficiente devido a características peculiares do FDTD, como a de requerer um passo de tempo bem inferior ao exigido pelo critério de Nyquist. A expressão da série DFT é dada por [53]:

$$
\begin{gathered}
G(m . \delta f)=\delta t_{D F T} \sum_{n=0}^{N_{D F T}-1} g\left(n \delta t_{D F T}\right) \exp \left[\frac{-j 2 \pi m n}{N_{D F T}}\right], \\
m=0 ; 1 ; 2 ; \ldots ; N_{\mathrm{DFT}}-1,
\end{gathered}
$$

na qual

- $m$ é o índice de freqüências, $m=0,1,2, \ldots, N_{\mathrm{DFT}}-1$. 
- $\quad \delta f$ é a resolução espectral dos cálculos no domínio da freqüência.

- $G(m . \delta f)$ é o valor complexo do módulo e fase da senóide componente em regime estacionário na freqüência $m . \delta f$.

- $\delta t_{D F T}$ é o período de amostragem do DFT e pode equivaler a um ou mais passos de tempo do FDTD $(\delta t)$.

- $g\left(n . \delta t_{D F T}\right)$ é o valor no domínio do tempo do pulso no instante $n . \delta t_{D F T}$ (sinal analisado)

- $\mathrm{N}_{\mathrm{DFT}}$ é o comprimento da somatória DFT. $\mathrm{N}_{\mathrm{DFT}}=1 /\left(\delta f . \delta t_{D F T}\right)$

Esta somatória atualiza os valores de $G(m . \delta f)$ a cada $\left(\delta t_{D F T} / \delta t\right)$ passos de tempo do FDTD. Isto consiste em outra vantagem em relação ao FFT, que exige armazenamento de todo o registro temporal com a finalidade de usá-lo como parâmetro de entrada de uma rotina FFT após a simulação FDTD estar concluída. Dependendo do número de pontos a serem analisados, isto pode acarretar um alto consumo de memória RAM ou de disco rígido.

A DFT também pode ser aplicada no cálculo do índice efetivo modal para excitações monocromáticas. Neste caso, $t$ (variável temporal) passa a ser $z$ (direção de propagação) e a freqüência $(f)$ calculada passa a ser $1 / \lambda_{\mathrm{g}}$, onde $\lambda_{\mathrm{g}}$ é o comprimento de onda guiado do modo. Dessa forma, a constante de fase modal pode ser calculada por $\beta=2 \pi / \lambda_{\mathrm{g}}$. Esta pode ser uma boa alternativa para a medição do índice efetivo, inclusive para problemas multimodo, se uma extensão longitudinal razoável de guia puder ser considerada.

\subsection{Condições de contorno na borda da janela computacional}

A fim de evitar reflexões indesejáveis nos limites do domínio espacial numérico, e devido à diversidade dos materiais que podem ser incluídos no contexto deste trabalho, optou-se pelo estudo e utilização da condição de contorno do tipo Camada Perfeitamente Casada Independente de Material (GMIPML) [26]. Diferente da condição de contorno proposta por Berenger [21], os campos $\bar{D}$ e $\bar{B}$ são aqui diretamente absorvidos pelo uso de condutividades $\sigma^{D}$ e $\sigma^{B}$, respectivamente, na região da Camada Perfeitamente Casada (Perfectly Matched Layer ou PML). Isto 
torna possível implementar a técnica absorvente separadamente das relações constitutivas do meio. A formulação geral é dada por [26]:

$$
\begin{aligned}
& \frac{\partial D_{x y}}{\partial t}+\sigma_{y}^{D} D_{x y}=\frac{\partial H_{z}}{\partial y}, \\
& \frac{\partial D_{x z}}{\partial t}+\sigma_{z}^{D} D_{x z}=-\frac{\partial H_{y}}{\partial z}, \\
& \frac{\partial D_{y z}}{\partial t}+\sigma_{z}^{D} D_{y z}=\frac{\partial H_{x}}{\partial z} \\
& \frac{\partial D_{y x}}{\partial t}+\sigma_{x}^{D} D_{y x}=-\frac{\partial H_{z}}{\partial x} \\
& \frac{\partial D_{z x}}{\partial t}+\sigma_{x}^{D} D_{z x}=\frac{\partial H_{y}}{\partial x} \\
& \frac{\partial D_{z y}}{\partial t}+\sigma_{y}^{D} D_{z y}=-\frac{\partial H_{x}}{\partial y} \\
& \frac{\partial B_{x y}}{\partial t}+\sigma_{y}^{B} B_{x y}=-\frac{\partial E_{z}}{\partial y} \\
& \frac{\partial B_{x z}}{\partial t}+\sigma_{z}^{B} B_{x z}=\frac{\partial E_{y}}{\partial z} \\
& \frac{\partial B_{y z}}{\partial t}+\sigma_{z}^{B} B_{y z}=-\frac{\partial E_{x}}{\partial z} \\
& \frac{\partial B_{y x}}{\partial t}+\sigma_{x}^{B} B_{y x}=\frac{\partial E_{z}}{\partial x} \\
& \frac{\partial B_{z y}}{\partial t}+\sigma_{x}^{B} B_{z x}=-\frac{\partial E_{y}}{\partial x} \\
& =\frac{\partial E_{x}}{\partial y}
\end{aligned}
$$

A condição de perfeito casamento na interface entre a região absorvente e o domínio do problema é dada por $\sigma_{u}^{D}=\sigma_{u}^{B} \quad(u=x, y, z)$. 
Um problema bidimensional no plano $x z$, por exemplo, apresenta a configuração ilustrada na Fig. 3. A Tabela 1 mostra a associação de condutividades em cada região.

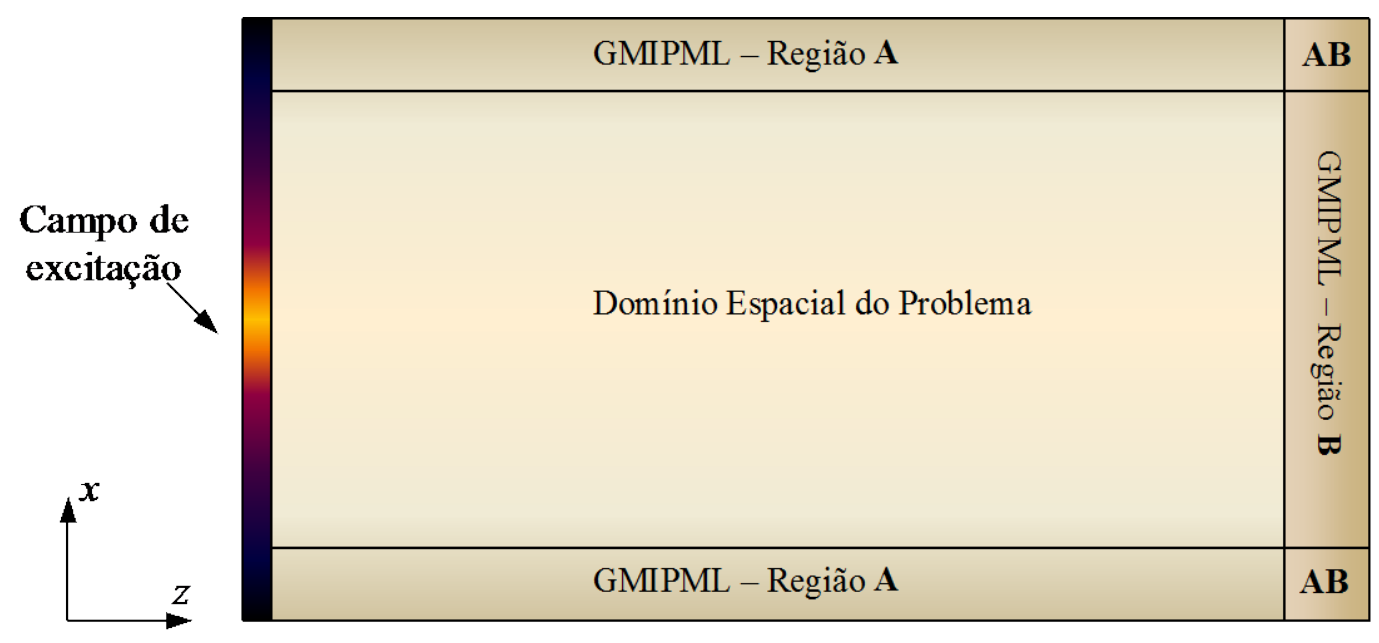

Fig. 3 - Disposições das camadas absorventes (GMIPML) num problema bidimensional

TABELA 1 - Propriedades das regiões no contexto da GMIPML

\begin{tabular}{|c|c|}
\hline Região & Características \\
\hline \hline $\mathrm{A}$ & $\sigma_{y}^{D, B}=\sigma_{z}^{D, B}=0, \quad \sigma_{x}^{D, B} \neq 0$. \\
\hline $\mathrm{B}$ & $\sigma_{y}^{D, B}=\sigma_{x}^{D, B}=0, \quad \sigma_{z}^{D, B} \neq 0$. \\
\hline $\mathrm{AB}$ & Apenas $\sigma_{y}^{D, B}=0$ \\
\hline Domínio espacial do problema & $\sigma_{y}^{D, B}=\sigma_{z}^{D, B}=\sigma_{x}^{D, B}=0$ \\
\hline
\end{tabular}

Particularizando (2.11)-(2.16) para a configuração bidimensional da Fig. 3 $(\partial / \partial y=0)$, e para a análise da propagação de modos TM (componentes $H_{y}, E_{x}$ e $\left.E_{z}\right)$, obtém-se para a região $\mathrm{AB}$ (quina):

$$
\begin{gathered}
\frac{\partial D_{x}}{\partial t}+\sigma_{z}^{D} D_{x}=-\frac{\partial H_{y}}{\partial z} \\
\frac{\partial D_{z}}{\partial t}+\sigma_{x}^{D} D_{z}=\frac{\partial H_{y}}{\partial x}
\end{gathered}
$$




$$
\begin{gathered}
\frac{\partial B_{y z}}{\partial t}+\sigma_{z}^{B} B_{y z}=-\frac{\partial E_{x}}{\partial z} \\
\frac{\partial B_{y x}}{\partial t}+\sigma_{x}^{B} B_{y x}=\frac{\partial E_{z}}{\partial x}
\end{gathered}
$$

Na aplicação do método FDTD, as equações acima podem ser discretizadas diretamente no tempo. Contudo, a precisão da solução numérica pode ser comprometida na medida em que $\sigma_{x}^{D, B}$ e $\sigma_{z}^{D, B}$ assumem valores relativamente altos. Neste caso, o decaimento dos campos é tão rápido que o algoritmo padrão de discretização temporal não pode ser facilmente utilizado [54]. Uma alternativa para conseguir expressões discretizadas mais precisas para as equações acima é tratá-las como equações diferenciais ordinárias de primeira ordem, encontrando suas soluções homogênea e particular. A solução homogênea para (2.17) é

$$
D_{x h o m o g}=C \exp \left(-\sigma_{z}^{D} t\right)
$$

na qual $C$ é uma constante. A solução recursiva para (2.20), ou seja, a expressão do valor de $D_{x}$ no instante atual em função de seu valor no instante anterior é

$$
D_{x}^{n+1}{ }_{\text {homog }}=\exp \left(-\sigma_{z}^{D} \delta t\right) D_{x h o m o g}^{n}
$$

A solução particular de (2.17) é dada por

$$
D_{x_{\text {part }}}=-\frac{1}{\sigma_{z}^{D}} \frac{\partial H_{y}}{\partial z}+K \exp \left(-\sigma_{z}^{D} t^{\prime}\right)
$$

Esta solução resulta somente da contribuição do rotacional de $\bar{H}$ dentro do incremento de tempo atual. O tempo $t^{\prime}$, por sua vez, é medido a partir do início do incremento atual. A constante $K$ (em relação ao tempo) pode ser determinada pela análise da solução particular no início do incremento temporal, ou seja, para $t^{\prime}=0$ [2]:

$$
\left.D_{x_{\text {part }}}\right|_{t^{\prime}=0}=0=-\frac{1}{\sigma_{z}^{D}} \frac{\partial H_{y}}{\partial z}+K \Rightarrow K=\frac{1}{\sigma_{z}^{D}} \frac{\partial H_{y}}{\partial z} .
$$


Ao final do incremento, $t^{\prime}=\delta t \mathrm{e}$

$$
\left.D_{x_{\text {part }}}\right|_{t^{\prime}=\delta t}=\frac{1}{\sigma_{z}^{D}} \frac{\partial H_{y}}{\partial z}\left[\exp \left(-\sigma_{z}^{D} \delta t\right)-1\right]
$$

Dessa forma, a solução geral pode ser obtida a partir de (2.21) e (2.24):

$$
D_{x}^{n+1}\left(i+\frac{1}{2}, k\right)=\exp \left(-\sigma_{z}^{D} \delta t\right) \cdot D_{x\left(i+\frac{1}{2}, k\right)}^{n}+\frac{1}{\sigma_{z}^{D}}\left[\exp \left(-\sigma_{z}^{D} \delta t\right)-1\right]-\left(\frac{H_{y}^{n+\frac{1}{2}}\left(i+\frac{1}{2}, k+\frac{1}{2}\right)-H_{y}^{n+\frac{1}{2}}\left(i+\frac{1}{2}, k-\frac{1}{2}\right)}{\delta z}\right)
$$

na qual a derivada espacial é calculada com base na célula de Yee bidimensional no plano $x z$ (Fig. 5).

Analogamente, resolvendo as equações (2.18)-(2.19b), obtém-se

$$
\begin{aligned}
& D_{z}^{n+1}\left(i, k+\frac{1}{2}\right)=\exp \left(-\sigma_{x}^{D} \delta t\right) \cdot D_{z\left(i, k+\frac{1}{2}\right)}^{n}- \\
& \frac{1}{\sigma_{x}^{D}}\left[\exp \left(-\sigma_{x}^{D} \delta t\right)-1\right]\left(\frac{H_{y}^{n+\frac{1}{2}}\left(i+\frac{1}{2}, k+\frac{1}{2}\right)}{-H_{y}^{n+\frac{1}{2}}\left(i-\frac{1}{2}, k+\frac{1}{2}\right)}\right) \\
& B_{y z}^{n+\frac{1}{2}}\left(i+\frac{1}{2}, k+\frac{1}{2}\right)=\exp \left(-\sigma_{z}^{B} \delta t\right) \cdot B_{y z}^{n-\frac{1}{2}}{ }_{\left(i+\frac{1}{2}, k+\frac{1}{2}\right)^{+}}^{+} \\
& \frac{1}{\sigma_{z}^{B}}\left[\exp \left(-\sigma_{z}^{B} \delta t\right)-1\right]-\left(\frac{E_{x\left(i+\frac{1}{2} 1, k+1\right)}^{n}-E_{x\left(i+\frac{1}{2}, k\right)}^{n}}{\delta z}\right) \\
& \left.B_{y x}^{n+\frac{1}{2}}{ }_{\left(i+\frac{1}{2}, k+\frac{1}{2}\right)}=\exp \left(-\sigma_{x}^{B} \delta t\right) \cdot B_{y x}^{n-\frac{1}{2}}{ }_{\left(i+\frac{1}{2}, k+\frac{1}{2}\right)}\right)^{-} \\
& \frac{1}{\sigma_{x}^{B}}\left[\exp \left(-\sigma_{x}^{B} \delta t\right)-1\right]-\left(\frac{E_{z\left(i+1, k+\frac{1}{2}\right)}^{n}-E_{z\left(i, k+\frac{1}{2}\right)}^{n}}{\delta x}\right)
\end{aligned}
$$

Conforme explicado a seguir, os valores de $\sigma_{x}^{D, B}$ e $\sigma_{z}^{D, B}$ variam ao longo do eixo $x$ e $z$, respectivamente. Sendo assim, estes parâmetros são caracterizados da seguinte forma:

$$
\sigma_{x}^{D}=\sigma_{x}^{D}(i), \quad \sigma_{x}^{B}=\sigma_{x}^{B}\left(i+\frac{1}{2}\right), \quad \sigma_{z}^{D}=\sigma_{z}^{D}(k) \quad \text { e } \quad \sigma_{z}^{B}=\sigma_{z}^{B}\left(k+\frac{1}{2}\right) .
$$


As fórmulas discretizadas para as regiões A e B podem ser obtidas a partir das equações (2.17)-(2.19b) e da TABELA 1. As equações simplificadas pela anulação de suas respectivas condutividades são discretizadas pelo procedimento tradicional.

Teoricamente, a condição $\sigma_{u}^{D}=\sigma_{u}^{B}$ garante o perfeito casamento na interface com o domínio do problema. Contudo, variações bruscas na condutividade geram reflexões numéricas [26], o que leva a ser adotada uma distribuição de condutividade variando de zero (na fronteira interna) até um valor máximo (na periferia da PML) ao longo de uma camada com $\mathrm{Npml}$ células. Quanto maior $\mathrm{Npml}$, menos abrupta será a variação de condutividade e mais eficiente será a performance da PML. Porém, a quantidade de memória e processamento destinados à camada absorvente também serão maiores, fator que deve ser levado em conta na implementação da PML.

Na direção $x$ (transversal), a função de distribuição de condutividade na PML é dada por $\sigma_{x}^{D, B}=a_{1}\left(x^{\prime}\right)^{p}$, na qual $p$ é a potência do perfil de condutividade, sendo $x^{\prime}=$ 0 na fronteira com o domínio interno e $x^{\prime}=\delta=N p m l . \delta x$ na extremidade da PML. O parâmetro $a_{1}$ é calculado a partir da expressão teórica para o fator de reflexão $R(\theta)$, o qual é dada por

$$
R(\theta)=\exp \left[-2\left(\cos \theta / c_{0}\right) \int_{0}^{\delta} \sigma_{x}^{D, B}(\rho) d \rho\right]
$$

Substituindo a expressão para $\sigma_{x}^{D, B}$ e $\theta=0$ (incidência normal) em (2.29), obtém-se

$$
a_{1}=-\frac{c_{0}(p+1) \ln [R(0)]}{2\left(\delta_{x} . n p m l\right)^{p+1}} .
$$

O procedimento é análogo para a direção $z$ (condutividade $\sigma_{z}^{D, B}$ ). 


\subsection{Caso particular: Guia planar não-linear e não-recíproco}

Nesta seção, o método FDTD é aplicado na análise da propagação de modos TM (componentes $H_{y}, E_{x}$ e $E_{z}$ ) no guia não-linear e não-recíproco ilustrado na Fig. 4. Trata-se de um problema bidimensional, pois não há variação dos campos ao longo do eixo $y$ (a direção propagação se dá no eixo $z$ ). $\mathrm{O}$ efeito de não-reciprocidade não é observado para modos TE em virtude da direção adotada para o campo $\mathrm{H}_{\mathrm{DC}}$.

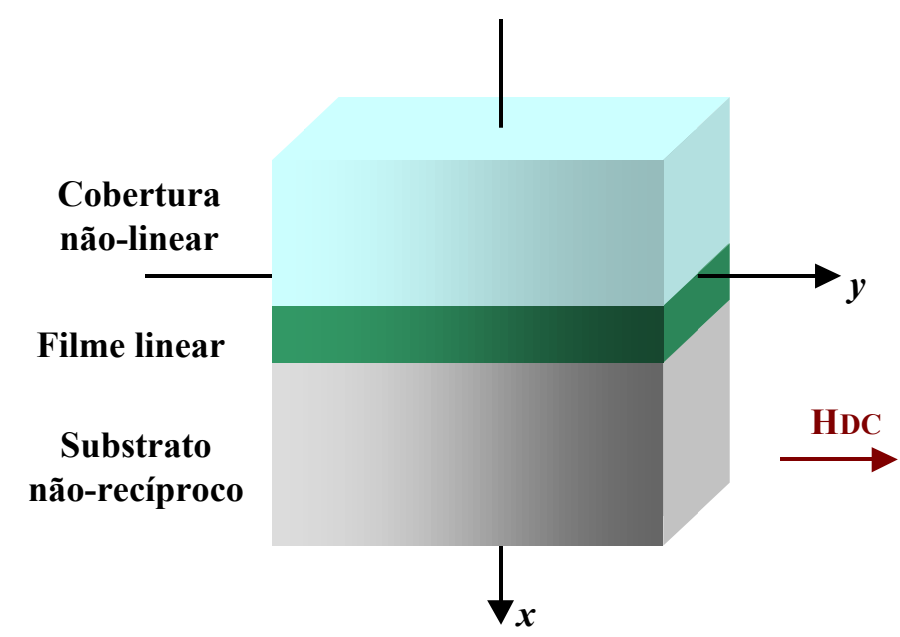

Fig. 4 - Guia planar analisado

O meio não-linear é do tipo Kerr cuja relação constitutiva para o campo elétrico é dada por

$$
\begin{aligned}
& D_{x}=\varepsilon_{0}\left[\varepsilon_{c}^{n l}+\alpha\left(\left|E_{x}\right|^{2}+\gamma\left|E_{z}\right|^{2}\right)\right] E_{x}, \\
& D_{z}=\varepsilon_{0}\left[\varepsilon_{c}^{n l}+\alpha\left(\left|E_{z}\right|^{2}+\gamma\left|E_{x}\right|^{2}\right)\right] E_{z},
\end{aligned}
$$

nas quais $\varepsilon_{c}^{n l}$ é a permissividade relativa estática, $\alpha$ é o coeficiente não-linear e $\gamma$ é um parâmetro que depende do mecanismo de não-linearidade. $\mathrm{O}$ substrato nãorecíproco é composto por material magnetoóptico, cuja anisotropia é controlada por um campo magnetostático $\left(\mathrm{H}_{\mathrm{DC}}\right)$ polarizado na direção $y$, situação na qual sua permissividade elétrica assume a seguinte característica tensorial: 


$$
\overline{\bar{\varepsilon}}^{n r}=\varepsilon_{0}\left[\begin{array}{ccc}
n_{x}^{2} & 0 & j \delta \\
0 & n_{y}^{2} & 0 \\
-j \delta & 0 & n_{z}^{2}
\end{array}\right],
$$

Onde $\delta$ é a constante magnetoóptica (adimensional), cujo valor indica o grau de nãoreciprocidade proporcionado pelo material magnetoóptico. Dessa forma, a relação constitutiva para o campo elétrico neste meio é escrita como

$$
\begin{gathered}
D_{x}=\varepsilon_{0}\left(n_{x}^{2} E_{x}+j \delta E_{z}\right), \\
D_{z}=\varepsilon_{0}\left(-j \delta E_{x}+n_{z}^{2} E_{z}\right),
\end{gathered}
$$

A relação constitutiva para o campo magnético é dada por $\bar{B}=\mu \bar{H}$ em todos os meios. A célula de Yee adaptada para um problema bidimensional no plano $x z$ é mostrada na Fig. 5.

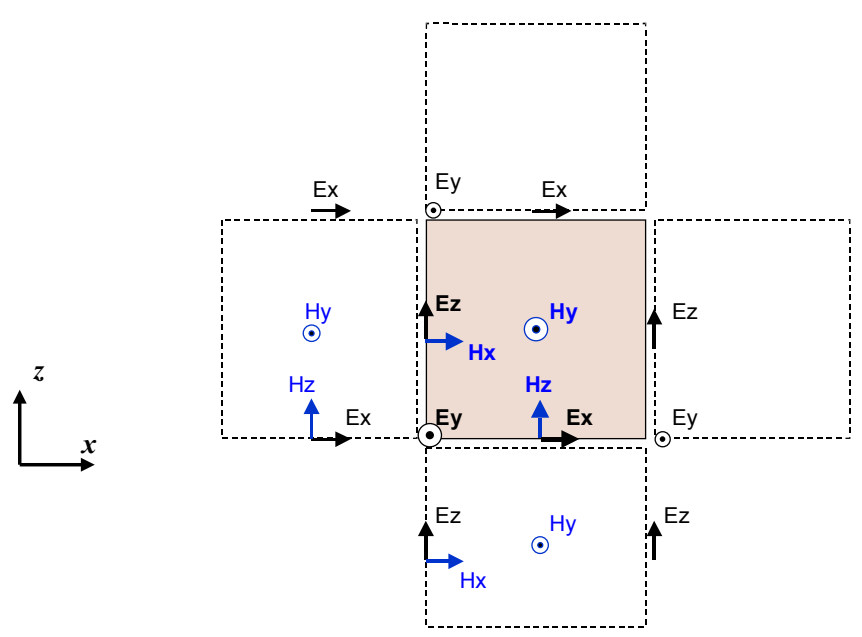

Fig. 5 - Célula de Yee bidimensional (plano $x z$ )

De posse das relações constitutivas e do formato de célula, as soluções recursivas para a utilização do método FDTD podem ser especificadas. Assim, tomando-se (2.3) e (2.4) como referência, tem-se

$$
\begin{aligned}
H_{y}^{n+\frac{1}{2}}\left(I+\frac{1}{2}, K+\frac{1}{2}\right)= & H_{y}^{n-\frac{1}{2}}\left(I+\frac{1}{2}, K+\frac{1}{2}\right)- \\
& \frac{\delta t}{\mu_{0}}\left(\frac{E_{x\left(I+\frac{1}{2}, K+1\right)}^{n}-E_{x\left(I+\frac{1}{2}, K\right)}^{n}}{\delta z}-\frac{\left.E_{z\left(I+1, K+\frac{1}{2}\right)}^{n}-E_{z\left(I, K+\frac{1}{2}\right)}^{n}\right)}{\delta x}\right)
\end{aligned}
$$




$$
\begin{aligned}
& D_{x}^{n+1}\left(I+\frac{1}{2}, K\right)=D_{x\left(I+\frac{1}{2}, K\right)}^{n}-\delta t \frac{H_{y}^{n+\frac{1}{2}}\left(I+\frac{1}{2}, K+\frac{1}{2}\right)-H_{y}^{n+\frac{1}{2}}\left(I+\frac{1}{2}, K-\frac{1}{2}\right)}{\delta z} \\
& D_{z}^{n+1}\left(I, K+\frac{1}{2}\right)=D_{z\left(I, K+\frac{1}{2}\right)}^{n}+\delta t \frac{H_{y}^{n+\frac{1}{2}}\left(I+\frac{1}{2}, K+\frac{1}{2}\right)-H_{y}^{n+\frac{1}{2}}\left(I-\frac{1}{2}, K+\frac{1}{2}\right)}{\delta x}
\end{aligned}
$$

Com base em (2.31) e (2.32), a solução para o campo elétrico no instante $\mathrm{t}=(n+1) \delta t$ no meio não-linear é dada por

$$
\begin{aligned}
& E_{x}^{n+1}\left(I+\frac{1}{2}, K\right)=\frac{D_{x}^{n+1}\left(I+\frac{1}{2}, K\right)}{\varepsilon_{0}\left[\varepsilon_{c}^{n l}+\alpha\left(\left|\widetilde{E}_{x}^{n+1}\left(I+\frac{1}{2}, K\right)\right|^{2}+\gamma\left|\widetilde{E}_{z}^{n+1}\left(I+\frac{1}{2}, K\right)\right|^{2}\right)\right]} \\
& E_{z}^{n+1}\left(I, K+\frac{1}{2}\right)=\frac{D_{z}^{n+1}\left(I, K+\frac{1}{2}\right)}{\varepsilon_{0}\left[\varepsilon_{c}^{n l}+\alpha\left(\left|\widetilde{E}_{z}^{n+1}\left(I, K+\frac{1}{2}\right)\right|^{2}+\gamma\left|\widetilde{E}_{x}^{n+1}\left(I, K+\frac{1}{2}\right)\right|^{2}\right)\right]}
\end{aligned}
$$

No meio não-linear, a permissividade elétrica depende dos valores instantâneos das componentes $E_{x}$ e $E_{z}$. Dessa forma, um procedimento iterativo é utilizado nessa região para garantir a convergência da solução, sendo que $\widetilde{E}_{x}^{n+1}$ e $\widetilde{E}_{z}^{n+1}$ recebem inicialmente o valor de $E_{x}^{n}$ e $E_{z}^{n}$ (passo de tempo anterior), respectivamente. As equações (2.39) e (2.40) são então iteradas até que o seguinte critério de convergência seja atingido:

$$
\frac{\left|E_{u}^{n+1}-\widetilde{E}_{u}^{n+1}\right|}{\left|\widetilde{E}_{u}^{n+1}\right|}<\delta_{t o l} \quad(u=x, z)
$$

Para o meio magnetoóptico, a solução para o campo elétrico, obtida a partir de (2.34) e (2.35), é dada por

$$
E_{x}^{n+1}\left(I+\frac{1}{2}, K\right)=\frac{1}{\varepsilon_{0}}\left(\frac{n_{y}^{2}}{n_{x}^{2} n_{y}^{2}-\delta^{2}} D_{x}^{n+1}\left(I+\frac{1}{2}, K\right)-\frac{j \delta}{n_{x}^{2} n_{y}^{2}-\delta^{2}} D_{z}^{n+1}\left(I+\frac{1}{2}, K\right)\right)
$$




$$
E_{z}^{n+1}\left(I, K+\frac{1}{2}\right)=\frac{1}{\varepsilon_{0}}\left(\frac{j \delta}{n_{x}^{2} n_{y}^{2}-\delta^{2}} D_{x}^{n+1}\left(I, K+\frac{1}{2}\right)+\frac{n_{x}^{2}}{n_{x}^{2} n_{y}^{2}-\delta^{2}} D_{z}^{n+1}\left(I, K+\frac{1}{2}\right)\right)
$$

Observe que $D_{z}^{n+1}$ em (2.41) e $D_{x}^{n+1}$ em (2.42) não estão referenciados em sua posição original na célula da Fig. 5. Dessa forma, são utilizadas as seguintes aproximações:

$$
\begin{aligned}
& D_{z}^{n+1}{ }_{\left(I+\frac{1}{2}, K\right)} \approx \frac{1}{4}\left\lfloor D_{z}^{n+1}{ }_{\left(I, K+\frac{1}{2}\right)}+D_{z}^{n+1}{ }_{\left(I+1, K+\frac{1}{2}\right)}+D_{z}^{n+1}{ }_{\left.I, K-\frac{1}{2}\right)}+D_{z}^{n+1}\left(I+1, K-\frac{1}{2}\right)\right\rfloor \\
& D_{x}^{n+1}\left(I, K+\frac{1}{2}\right) \approx \frac{1}{4}\left\lfloor D_{x}^{n+1}\left(I+\frac{1}{2}, K\right)+D_{x}^{n+1}\left(I+\frac{1}{2}, K+1\right)+D_{x}^{n+1}\left(I-\frac{1}{2}, K\right)+D_{x}^{n+1}\left(I-\frac{1}{2}, K+1\right)\right\rfloor
\end{aligned}
$$

A distribuição de células ao longo do eixo $x$ é mostrada na Fig. 6, na qual $\delta x=$ $0,046 \mu \mathrm{m}$. Na direção longitudinal, $z$, foi usado um total de 10040 células, sendo que $\delta z=0,01 \mu \mathrm{m}$, resultando numa distância de propagação total de 100,4 $\mu \mathrm{m}$.

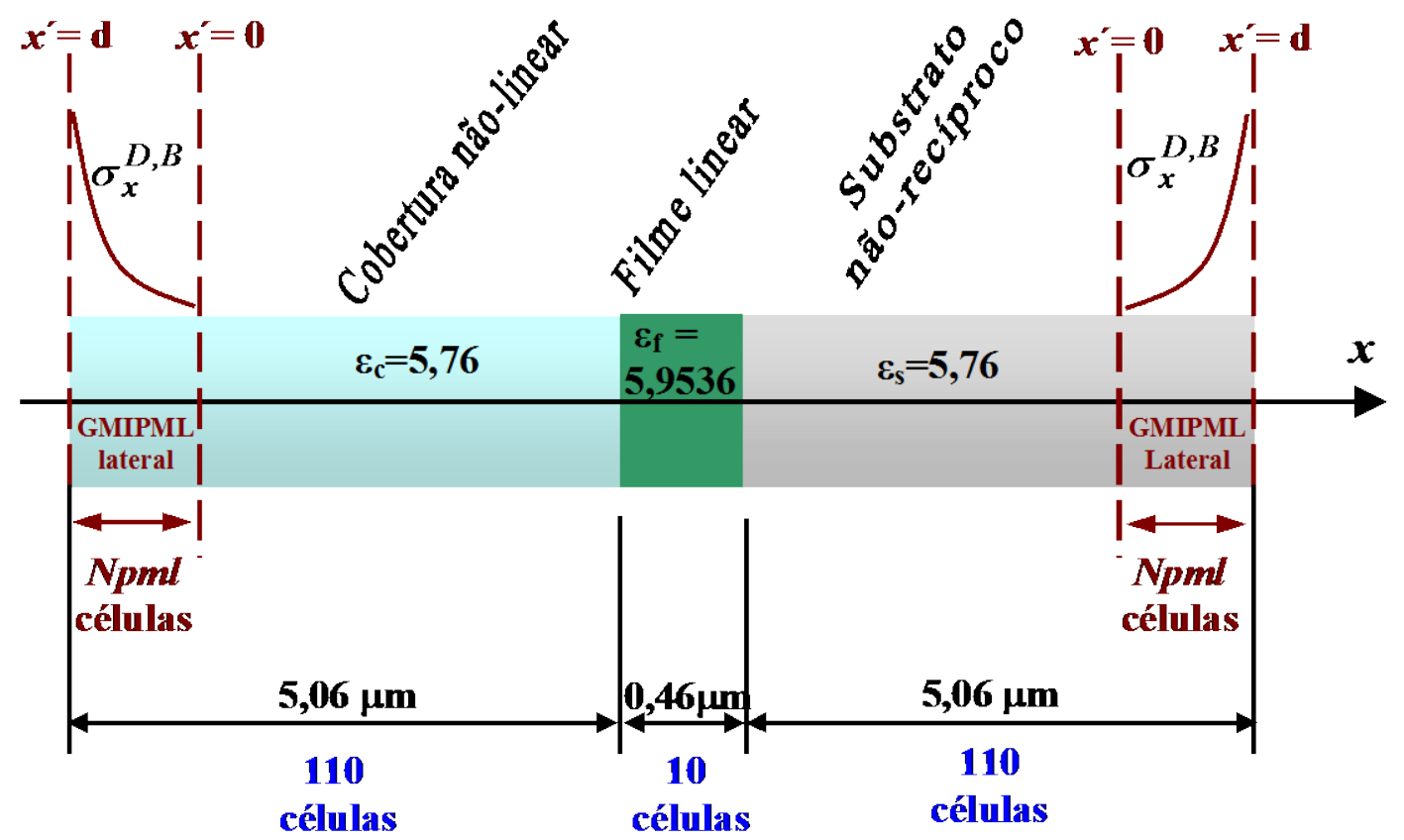

Fig. 6 - Discretização espacial ao longo da direção transversal, x.

Os valores dos parâmetros dos materiais são mostrados na TABELA 2. O comprimento de onda utilizado é $\lambda=1,152 \mu \mathrm{m}$. 
TABELA 2 - Parâmetros dos materiais

\begin{tabular}{|c|c|}
\hline Descrição & Valor \\
\hline \hline Índice de refração do filme, $n_{f}\left(=\sqrt{\varepsilon_{f}}\right)$ & 2,44 \\
\hline Índice de refração do substrato magnetoóptico, $n_{s}\left(=\sqrt{\varepsilon_{s}}\right)$ & 2,40 \\
\hline Índice de refração (estático) do meio não-linear, $n_{c}\left(=\sqrt{\varepsilon_{c}}\right)$ & 2,40 \\
\hline Coeficiente não-linear, $\alpha$ & $0,6377 \times 10^{-11} \mathrm{~m}^{2} / \mathrm{V}^{2}$ \\
\hline Constante de acoplamento não-linear, $\gamma$ & $-1 / 2$ \\
\hline
\end{tabular}

O passo de tempo é calculado por intermédio do critério de estabilidade de Courant, que neste caso é dado por:

$$
\delta t_{\text {max }}=\frac{n_{\text {min }}}{c_{0} \sqrt{\left(\frac{1}{\delta x}\right)^{2}+\left(\frac{1}{\delta z}\right)^{2}}} .
$$

Para a discretização apresentada, $\delta t_{\max }=7,82282 \times 10^{-2} f s$. Foram utilizados 12040 passos de tempo, resultando em um tempo total de propagação de $941,8678 \mathrm{fs}$. A excitação inicial é a solução analítica para a distribuição transversal do modo TM do guia linear e isotrópico correspondente, a qual é multiplicada por uma função temporal harmônica na freqüência de operação, $f_{0}=c_{0} / \lambda$. A técnica de parede absorvente GMPIML é implementada para modos TM, conforme descrito na Seção 2.5, sendo que o número de células utilizadas nas camadas é $\mathrm{Npml}=30$. Um pequeno teste demonstrando a atuação da GMIPML é ilustrado na Fig. 7, na qual está representado o modo propagante $\mathrm{TM}_{0}$ da estrutura mostrada na Fig. 6. Esta estrutura foi excitada com uma densidade de potência óptica $P_{\text {exc }}=125 \mathrm{~mW} / \mathrm{mm}$. Com relação à discretização, foi utilizado um número reduzido de células ao longo da direção de propagação (400 células, ou $4 \mu \mathrm{m}$ ) e um número suficiente de passos de tempo para a onda incidir no limite frontal. Na ausência da condição de contorno absorvente, a onda é totalmente refletida (Fig. 7a), enquanto que na aplicação da GMIPML (Fig. 7b), a onda é absorvida com eficiência. 

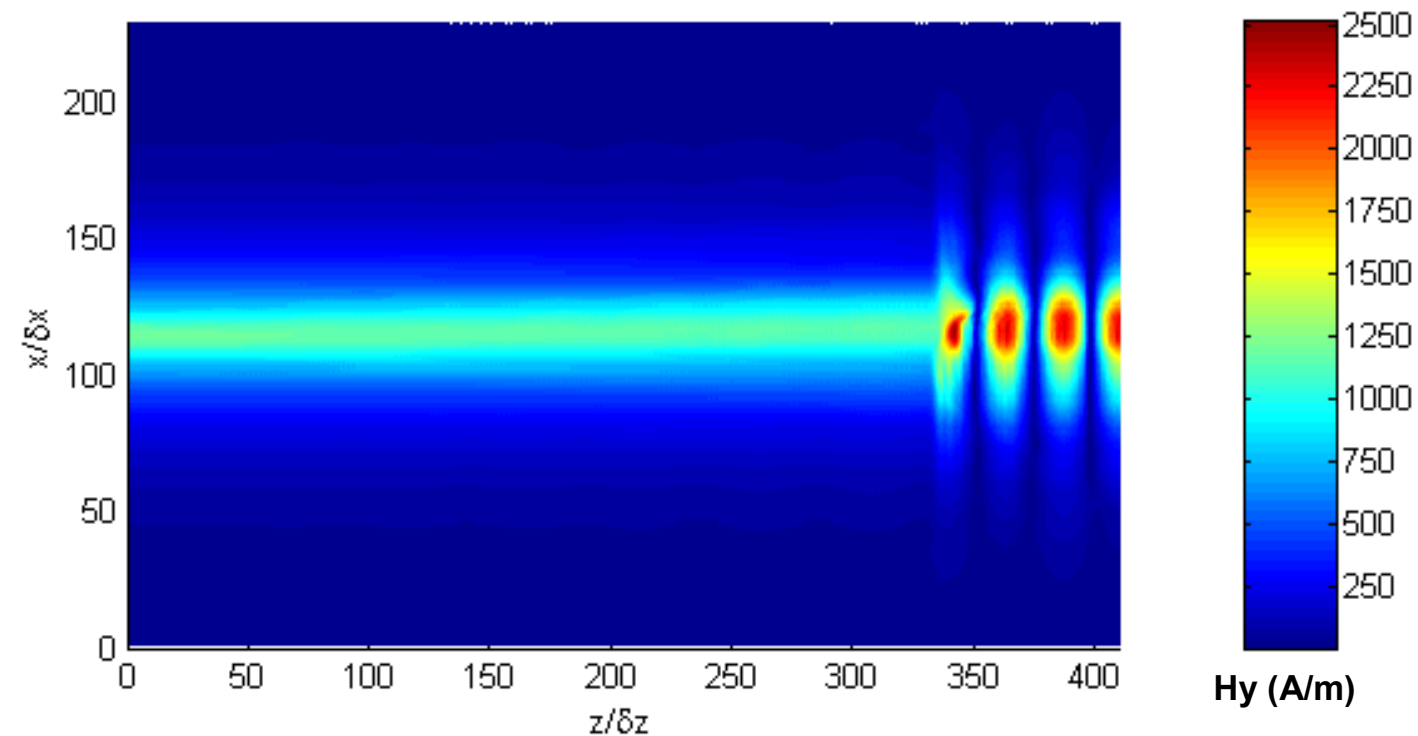

(a)
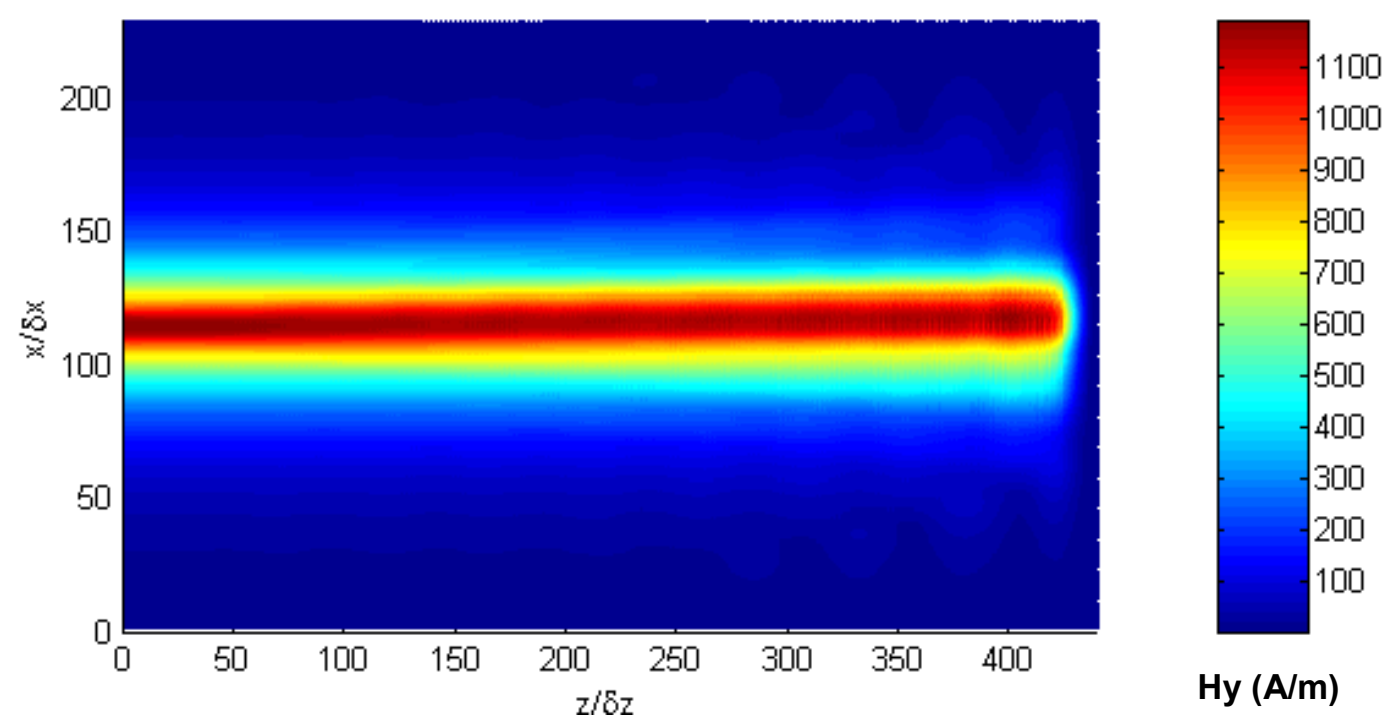

(b)

Fig. 7 - Onda incidindo no limite frontal após 500 passos de tempo: (a) na ausência de GMIPML $(\mathrm{Npml}=0)$ e $(\mathrm{b})$ com o domínio revestido pelas camadas absorventes $\left(n p m l=30, p=3\right.$ e $\left.R(0)=10^{-2}\right)$.

Os resultados referentes à análise da não-linearidade e não-reciprocidade para esta estrutura são mostrados na Seção 5.1 em conjunto com os resultados obtidos com o FD-BPM para a mesma estrutura. O formalismo FD-BPM é exposto no capítulo a seguir. 


\section{O MÉTODO FD-BPM BIDIMENSIONAL}

Este capítulo mostra o procedimento proposto na criação de um algoritmo unificado que possibilite a consideração de meios isotrópicos, anisotrópicos e nãolineares em dispositivos guias de ondas ópticos. A formulação inclui a condição de contorno absorvente PML (camada perfeitamente casada) [29]-[31] para o truncamento dos limites espaciais transversais, bem como a TBC (condição de contorno transparente) [32]. Esta última é uma alternativa bastante eficiente e não requer modificações adicionais nas equações de Maxwell.

\subsection{Expansão do formalismo FD-BPM}

Para um problema bidimensional no plano $x z$ e análise de modos TM (componentes $H_{y}, E_{x}$ e $E_{z}$ ), pode-se obter a partir das equações de Maxwell o seguinte conjunto de equações:

$$
\begin{gathered}
\mu_{0} \frac{\partial H_{y}}{\partial t}=-\left(\frac{\partial E_{x}}{\partial z}-\frac{\partial E_{z}}{\partial \widetilde{x}}\right) \\
\frac{\partial E_{x}}{\partial t}=-\xi_{x x} \frac{\partial H_{y}}{\partial z}+\xi_{x z} \frac{\partial H_{y}}{\partial \widetilde{x}} \\
\frac{\partial E_{z}}{\partial t}=\xi_{x z} \frac{\partial H_{y}}{\partial z}+\xi_{z z} \frac{\partial H_{y}}{\partial \widetilde{x}}
\end{gathered}
$$

Nestas equações, as derivadas são tomadas em relação ao eixo $x$ transformado ( $\widetilde{x}$ ) em função da condutividade da PML. A transformação só é necessária no eixo $x$ pois no caso do BPM bidimensional, camadas de PML frontais e de canto não são usadas (para o BPM, apenas os eixos transversais são mapeados). De acordo com [10]-[11], se $x$ é a coordenada normal à superfície da camada (PML), esta deve ser substituída nas equações de Maxwell pela variável espacial complexa $\tilde{x}$, 


$$
\tilde{x}(\tau)=\int_{0}^{d}\left(1-j \frac{\sigma_{H}(\tau)}{\omega \mu_{0}}\right) d \tau,
$$

na qual $\sigma_{\mathrm{H}}(x)$ e $d$ são o perfil de condutividade magnética e a espessura da PML, respectivamente. Na Equação (3.4), para evitar a complexidade da permissividade elétrica dos meios envolvidos na implementação da PML, optou-se por utilizar $\sigma_{H} / \mu_{0}$ ao invés de $\sigma_{E} / \varepsilon$ (onde $\sigma_{\mathrm{E}}$ é a condutividade elétrica), o que está de acordo com a condição de casamento entre a PML e o meio adjacente, pela qual $\sigma_{\mathrm{H}} / \mu_{0}=\sigma_{\mathrm{E}} / \varepsilon$. Mapeamento análogo ao feito em (3.4) é discutido em [31], no qual destaca-se o fato que este tipo de transformação é transparente às relações constitutivas do meio.

Os elementos $\xi_{\mathrm{uv}}$ estão relacionados com a inversa da matriz permissividade elétrica. A TABELA 3 mostra as expressões para $\xi_{\text {uv }}$ em cada meio envolvido.

TABELA 3 - Elementos da matriz permissividade inversa.

\begin{tabular}{|c|c|c|c|}
\hline & Isotrópico & Magnetoóptico & \multicolumn{2}{|c|}{ Não-linear } \\
\hline$\xi_{\mathrm{xx}}$ & $\frac{1}{\varepsilon_{0} n^{2}}$ & $\frac{1}{\varepsilon_{0}} \frac{n_{z}^{2}}{n_{x}^{2} n_{z}^{2}-\delta^{2}}$ & $\frac{1}{\varepsilon_{0} \varepsilon_{x x}^{n l}}=\frac{1}{\varepsilon_{0}\left[\varepsilon_{c}^{n l}+\alpha\left(\left|E_{x}\right|^{2}+\gamma\left|E_{z}\right|^{2}\right)\right]}$ \\
\hline$\xi_{\mathrm{zz}}$ & $\frac{1}{\varepsilon_{0} n^{2}}$ & $\frac{1}{\varepsilon_{0}} \frac{n_{x}^{2}}{n_{x}^{2} n_{z}^{2}-\delta^{2}}$ & $\frac{1}{\varepsilon_{0} \varepsilon_{z z}^{n l}}=\frac{1}{\varepsilon_{0}\left[\varepsilon_{c}^{n l}+\alpha\left(\left|E_{z}\right|^{2}+\gamma\left|E_{x}\right|^{2}\right)\right]}$ \\
\hline$\xi_{\mathrm{xz}}$ & 0 & $-\frac{1}{\varepsilon_{0}} \frac{j \delta}{n_{x}^{2} n_{z}^{2}-\delta^{2}}$ & 0 \\
\hline
\end{tabular}

Foi considerado que o campo magnetostático $\left(H_{D C}\right)$ está aplicado ao longo da direção $y$, o que resulta na seguinte expressão para a permissividade do meio magnetoóptico

$$
\overline{\bar{\varepsilon}}^{n r}=\varepsilon_{0}\left[\begin{array}{ccc}
n_{x}^{2} & 0 & j \delta \\
0 & n_{y}^{2} & 0 \\
-j \delta & 0 & n_{z}^{2}
\end{array}\right]
$$


na qual $\delta$ é a constante magnetoóptica.

Em relação ao meio não-linear (ver TABELA 3), $\gamma$ é um coeficiente que depende do mecanismo de não-linearidade [55], ou seja, $\gamma=1$ para efeitos térmicos ou eletrostricção, $\gamma=1 / 3$ para distorção eletrônica, e $\gamma=-1 / 2$ para orientação molecular. $\mathrm{O}$ parâmetro $\varepsilon_{c}^{n l}$ é a permissividade estática relativa do material nãolinear e $\alpha$ é o coeficiente não-linear, o qual é dado por $\alpha=\varepsilon_{c}^{n l} n_{2 s} c_{0} \varepsilon_{0}$. Aqui $c_{0}$ é a velocidade da luz no vácuo e $n_{2 s}=10^{-9} \mathrm{~m}^{2} / W$ (meio focalizante) [37].

De (3.1)-(3.3), obtém-se

$$
\mu_{0} \frac{\partial^{2} H_{y}}{\partial t^{2}}=\xi_{x x} \frac{\partial^{2} H_{y}}{\partial z^{2}}+\left(\frac{\partial \xi_{x x}}{\partial z}+\frac{\partial \xi_{x z}}{\partial \widetilde{x}}\right) \frac{\partial H_{y}}{\partial z}+\xi_{z z} \frac{\partial^{2} H_{y}}{\partial \tilde{x}^{2}}+\left(\frac{\partial \xi_{z z}}{\partial \tilde{x}}-\frac{\partial \xi_{x z}}{\partial z}\right) \frac{\partial H_{y}}{\partial \tilde{x}}
$$

Rescrevendo (3.6) em termos da coordenada $x$ original, obtém-se

$$
\begin{aligned}
\mu_{0} \frac{\partial^{2} H_{y}}{\partial t^{2}} & =\xi_{x x} \frac{\partial^{2} H_{y}}{\partial z^{2}}+\left(\frac{\partial \xi_{x x}}{\partial z}+v \frac{\partial \xi_{x z}}{\partial x}\right) \frac{\partial H_{y}}{\partial z}+ \\
& +\xi_{z z} v \frac{\partial}{\partial x}\left(v \frac{\partial H_{y}}{\partial x}\right)+v\left(v \frac{\partial \xi_{z z}}{\partial x}-\frac{\partial \xi_{x z}}{\partial z}\right) \frac{\partial H_{y}}{\partial x}
\end{aligned}
$$

na qual

$$
v=v(x)=\left(1-j \frac{\sigma_{H}(x)}{\omega \mu_{0}}\right)^{-1}
$$

A aproximação por envoltória de variação lenta (SVEA) é aplicada sobre a componente $H_{y}$, o que possibilitará a utilização de passos longitudinais maiores no modelo discretizado e a conseqüente redução do tempo de processamento. Portanto, a componente $H_{y}$ é escrita na forma $H_{y}(x, z, t)=\Psi(x, z) e^{j\left(\omega t-s n_{0} k_{0} z\right)}$, na qual $\Psi(x, z)$ é a envoltória de variação longitudinal lenta da componente $H_{y}, k_{0}$ é a constante de propagação no espaço livre, $n_{0}$ é um índice efetivo de referência, $\mathrm{s}=1$ para propagação no sentido $+z$ e $s=-1$ para propagação na direção $-z$. Neste caso, (3.6) torna-se 


$$
\begin{aligned}
& \frac{\partial^{2} \Psi}{\partial z^{2}}+\left(\frac{\partial \ln \xi_{x x}}{\partial z}+\frac{v}{\xi_{x x}} \frac{\partial \xi_{x z}}{\partial x}-2 j s \kappa\right) \frac{\partial \Psi}{\partial z}+ \\
& {\left[\frac{\xi_{z z}}{\xi_{x x}} v^{2} \frac{\partial^{2}}{\partial x^{2}}+\frac{v}{\xi_{x x}}\left(v \frac{\partial \xi_{z z}}{\partial x}-\frac{\partial \xi_{x z}}{\partial z}+\xi_{z z} \frac{\partial v}{\partial x}\right) \frac{\partial}{\partial x}-j s \kappa\left(\frac{\partial \ln \xi_{x x}}{\partial z}+\frac{v}{\xi_{x x}} \frac{\partial \xi_{x z}}{\partial x}\right)+\frac{\omega^{2} \mu_{0}}{\xi_{x x}}-\kappa^{2}\right] \Psi=0}
\end{aligned}
$$

sendo $\kappa=n_{0} k_{0}$.

A equação (3.9) pode ser escrita como

$$
\frac{\partial^{2} \Psi}{\partial z^{2}}-2 j \zeta \frac{\partial \Psi}{\partial z}+P \Psi=0,
$$

na qual $\zeta$ é uma variável escalar e $P$ é um operador, dados por

$$
\begin{gathered}
\zeta=s \kappa+\frac{j}{2}\left(\frac{\partial \ln \xi_{x x}}{\partial z}+\frac{v}{\xi_{x x}} \frac{\partial \xi_{x z}}{\partial x}\right), \\
P=\frac{\xi_{z z}}{\xi_{x x}} v^{2} \frac{\partial^{2}}{\partial x^{2}}+\frac{v}{\xi_{x x}}\left(v \frac{\partial \xi_{z z}}{\partial x}-\frac{\partial \xi_{x z}}{\partial z}+\xi_{z z} \frac{\partial v}{\partial x}\right) \frac{\partial}{\partial x} \\
-j s \kappa\left(\frac{\partial \ln \xi_{x x}}{\partial z}+\frac{v}{\xi_{x x}} \frac{\partial \xi_{x z}}{\partial x}\right)+\frac{\omega^{2} \mu_{0}}{\xi_{x x}}-\kappa^{2} .
\end{gathered}
$$

A equação (3.10) pode ser rescrita como

$$
\left[\frac{\partial}{\partial z}+j\left(\sqrt{P+\zeta^{2}}-\zeta\right)\right]\left[\frac{\partial}{\partial z}-j\left(\sqrt{P+\zeta^{2}}+\zeta\right)\right] \Psi=0
$$

Portanto, considerando apenas um sentido de propagação $(s=1$ para $+z$ e $s=-1$ para $-z$ ), a mesma equação (3.10) reduz-se a

$$
\frac{\partial \Psi}{\partial z}=-s j\left(\sqrt{P+\zeta^{2}}-s \zeta\right) \Psi
$$

Para resolver o radical existente em (3.13), são utilizados os aproximantes de Padé de ordem $(1,1)$. Dessa forma, 


$$
\sqrt{P+\zeta^{2}}-s \zeta \approx \frac{\frac{P}{2 s \zeta}}{1+\frac{P}{4 \zeta^{2}}}
$$

\subsubsection{Implementação do esquema Crank-Nicolson (CN)}

Este é o esquema mais usual, consistindo na aplicação de aproximações de primeira ordem nas derivadas espaciais de campo, as quais sempre são centradas para proporcionar um sistema numérico estável. Substituindo (3.14) na equação (3.13) aproximada por diferenças finitas, obtém-se

$$
\frac{\Psi_{i}^{m+1}}{\delta z}+\frac{1}{4 \zeta_{i}^{m+\frac{1}{2}}}\left(\frac{1}{\delta z \zeta_{i}^{m+\frac{1}{2}}}+j\right) P \Psi_{i}^{m+1}=\frac{\Psi_{i}^{m}}{\delta z}+\frac{1}{4 \zeta_{i}^{m+\frac{1}{2}}}\left(\frac{1}{\delta z \zeta_{i}^{m+\frac{1}{2}}}-j\right) P \Psi_{i}^{m}
$$

Expandindo (3.11), tem-se

$$
\zeta_{i}^{m+\frac{1}{2}}=s \kappa+\frac{j}{2}\left(\frac{\ln \xi_{x x}^{m+1}(i)-\ln \xi_{x x(i)}^{m}}{\delta z}+v_{i} \frac{\xi_{x z}^{m+1}{ }_{(i+1)}+\xi_{x z(i+1)}^{m}-\xi_{x z}^{m+1}{ }_{(i-1)}-\xi_{x z(i-1)}^{m}}{2 \delta x\left(\xi_{x x}^{m+1}{ }_{(i)}+\xi_{x x(i)}^{m}\right)}\right)
$$

A equação (3.15) deve ser rescrita na forma de um sistema linear. Assim

$$
\theta_{+}^{m+1} \Psi_{i+1}^{m+1}+\theta_{o}^{m+1} \Psi_{i}^{m+1}+\theta_{-}^{m+1} \Psi_{i-1}^{m+1}=\theta_{+}^{m} \Psi_{i+1}^{m}+\theta_{o}^{m} \Psi_{i}^{m}+\theta_{-}^{m} \Psi_{i-1}^{m},
$$

tal que $i$ varia de 1 a $N x$ (índice máximo de célula no eixo $x$ ), $m+1$ representa o passo longitudinal futuro, e

$$
\theta_{ \pm}^{p}=\frac{1}{4 \delta x^{2} \xi_{x x(i)}^{p}}\left[\frac{\operatorname{sgn}}{\delta z\left(\zeta_{i}^{m+\frac{1}{2}}\right)^{2}}+\frac{j}{\zeta_{i}^{m+\frac{1}{2}}}\right]\left[\left(v_{i} \pm \frac{v_{i+1}-v_{i-1}}{4}\right) \xi_{z z(i)}^{p} \pm v_{i} \frac{\xi_{z z(i+1)}^{p}-\xi_{z z(i-1)}^{p}}{4}\right]
$$


$\theta_{o}^{p}=\frac{\operatorname{sgn}}{\delta z}-\frac{1}{4}\left[\frac{\operatorname{sgn}}{\delta z\left(\zeta_{i}^{m+\frac{1}{2}}\right)^{2}}+\frac{j}{\zeta_{i}^{m+\frac{1}{2}}}\right]\left[\frac{2 \xi_{z z(i)}^{p} v_{i}^{2}}{\delta x^{2} \xi_{x x(i)}^{p}}+j s \kappa\left(\left.\frac{\partial \ln \xi_{x x}}{\partial z}\right|_{i} ^{p}+v_{i} \frac{\xi_{x z(i+1)}^{p}-\xi_{x z(i-1)}^{p}}{2 \delta x \xi_{x x(i)}^{p}}\right)-\frac{\mu \omega^{2}}{\xi_{x x(i)}^{p}}+\kappa^{2}\right]$,

sendo $\operatorname{sgn}=1$ se $p=m+1$ ou $\operatorname{sgn}=-1$, se $p=m$.

\subsubsection{Implementação do esquema Douglas Generalizado (GD)}

Este esquema consiste na aplicação de uma aproximação em diferenças finitas de ordem mais alta sobre a segunda derivada do campo em relação ao eixo transversal. Isto nos permite obter um truncamento mais preciso e menos sensível passo de discretização transversal adotado. Substituindo (3.14) em (3.13), obtém-se

$$
\left(1+\frac{P}{4 \zeta^{2}}\right) \frac{\partial \Psi}{\partial z}=-\frac{j}{2 \zeta} P \Psi
$$

O Esquema GD, aplicado na derivada segunda do campo em relação ao eixo transversal é caracterizado por,

$$
\frac{\delta^{2} \Psi}{\delta x^{2}}=\frac{1}{12}\left(\frac{\partial^{2} \Psi}{\partial x^{2}}\right)_{i+1}+\frac{5}{6}\left(\frac{\partial^{2} \Psi}{\partial x^{2}}\right)_{i}+\frac{1}{12}\left(\frac{\partial^{2} \Psi}{\partial x^{2}}\right)_{i-1} .
$$

A equação (3.18) pode ser rescrita como

$$
\begin{gathered}
\frac{\xi_{x x}}{\xi_{z z} v^{2}} P \Psi=2 j \zeta \frac{\xi_{x x}}{\xi_{z z} v^{2}}\left(1+\frac{P}{4 \zeta^{2}}\right) \frac{\partial \Psi}{\partial z} \Rightarrow \\
\frac{\partial^{2} \Psi}{\partial x^{2}}=2 j \zeta \frac{\xi_{x x}}{\xi_{z z} v^{2}}\left(1+\frac{P}{4 \zeta^{2}}\right) \frac{\partial \Psi}{\partial z}-\Theta \Psi,
\end{gathered}
$$

na qual

$$
\Theta=\left(\frac{\partial \ln \xi_{z z}}{\partial x}-\frac{1}{v \xi_{z z}} \frac{\partial \xi_{x z}}{\partial z}+\frac{\partial \ln v}{\partial x}\right) \frac{\partial}{\partial x}-\frac{1}{v^{2} \xi_{z z}}\left[j \kappa\left(\frac{\partial \xi_{x x}}{\partial z}+v \frac{\partial \xi_{x z}}{\partial x}\right)-\omega^{2} \mu_{0}+\kappa^{2} \xi_{x x}\right] .
$$

Substituindo (3.20) em (3.19), obtém-se 


$$
\begin{aligned}
& \frac{\delta^{2} \Psi}{\delta x^{2}}=\frac{1}{12}\left[2 j \zeta \frac{\xi_{x x}}{\xi_{z z} v^{2}}\left(1+\frac{P}{4 \zeta^{2}}\right) \frac{\partial \Psi}{\partial z}-\Theta \Psi\right]_{i+1} \\
& +\frac{5}{6}\left[2 j \zeta \frac{\xi_{x x}}{\xi_{z z} v^{2}}\left(1+\frac{P}{4 \zeta^{2}}\right) \frac{\partial \Psi}{\partial z}-\Theta \Psi\right]_{i} \\
& +\frac{1}{12}\left[2 j \zeta \frac{\xi_{x x}}{\xi_{z z} v^{2}}\left(1+\frac{P}{4 \zeta^{2}}\right) \frac{\partial \Psi}{\partial z}-\Theta \Psi\right]_{i-1} .
\end{aligned}
$$

Aplicando diferenças finitas na equação acima, obtém-se

$$
\frac{1}{\delta x^{2}}\left(\Psi_{i+1}^{m+1}-2 \Psi_{i}^{m+1}+\Psi_{i-1}^{m+1}+\Psi_{i+1}^{m}-2 \Psi_{i}^{m}+\Psi_{i-1}^{m}\right)=\frac{1}{6} Y_{i+1}^{m+\frac{1}{2}}+\frac{5}{3} Y_{i}^{m+\frac{1}{2}}+\frac{1}{6} Y_{i-1}^{m+\frac{1}{2}},
$$

na qual

$$
\begin{array}{r}
Y_{l}^{m+\frac{1}{2}}=2 j \zeta_{l}^{m+\frac{1}{2}} \frac{\left(\xi_{x x(l)}^{m+1}+\xi_{x x(l)}^{m}\right)}{\left.\left(\xi_{z z(l)}^{m+1}+\xi_{z z(l)}^{m}\right)\right)^{2}}\left(\frac{\Psi_{l}^{m+1}-\Psi_{l}^{m}}{\delta z}+\frac{1}{4\left(\zeta_{l}^{m+\frac{1}{2}}\right)^{2}} \frac{P \Psi_{l}^{m+1}-P \Psi_{l}^{m}}{\delta z}\right)-\Theta \Psi_{l}^{m+\frac{1}{2}} \\
(l=i-1, i \text { ou } i+1)
\end{array}
$$

Por via de um procedimento algébrico direto, porém extenso, é possível escrever (3.23) na forma de um sistema matricial pentadiagonal, tal que a distribuição transversal de campo em $\Psi^{m+1}$ é obtida a partir da mesma em $\Psi^{m}$. Por se tratar de um sistema pentadiagonal, um maior esforço numérico computacional é necessário para solucioná-lo, em comparação ao caso tridiagonal. Para tratar os problemas de truncamento do domínio transversal pode ser aplicada tanto a TBC quanto a PML.

\subsection{Método iterativo entre o campo elétrico e a permissividade elétrica na região não-linear}

Conforme mostrado na TABELA 3, as expressões para o tensor permissividade na região não-linear dependem da intensidade de campo, 


$$
\begin{aligned}
& \varepsilon_{x x}^{n l}=\varepsilon_{c}^{n l}+\alpha\left(\left|E_{x}\right|^{2}+\gamma\left|E_{z}\right|^{2}\right) \\
& \varepsilon_{z z}^{n l}=\varepsilon_{c}^{n l}+\alpha\left(\gamma\left|E_{x}\right|^{2}+\left|E_{z}\right|^{2}\right)
\end{aligned}
$$

Isto requer a adoção de um procedimento iterativo para a convergência do campo elétrico e para a distribuição de permissividade elétrica em $z=(m+1) \delta z$ quando a região não-linear é envolvida. A partir das equações de Maxwell, obtém-se

$$
\begin{gathered}
\left|E_{x}\right|^{2}=\frac{1}{\omega^{2} \varepsilon_{0}^{2}\left(\varepsilon_{x x}^{n l}\right)^{2}}\left|\kappa \Psi+j \frac{\partial \Psi}{\partial z}\right|^{2}=\frac{C_{x}}{\left(\varepsilon_{x x}^{n l}\right)^{2}}, \\
\left|E_{z}\right|^{2}=\frac{1}{\omega^{2} \varepsilon_{0}^{2}\left(\varepsilon_{z z}^{n l}\right)^{2}}\left|v \frac{\partial \Psi}{\partial x}\right|^{2}=\frac{C_{z}}{\left(\varepsilon_{z z}^{n l}\right)^{2}}
\end{gathered}
$$

onde $C_{x}$ e $C_{z}$ são constantes introduzidas para o único fim de compactação das equações. Suas formas discretizadas serão fornecidas mais adiante. Note que o parâmetro $v$, associado ao perfil de condutividade da PML, também é incorporado em (3.27). Substituindo (3.26) e (3.27) em (3.24) e (3.25), é possível combinar o sistema resultante na seguinte equação polinomial de nono grau para $\varepsilon_{x x}^{n l}$ :

$$
\begin{aligned}
& \left(\varepsilon_{x x}^{n l}\right)^{9}+(2 \gamma-3) \varepsilon_{c}^{n l}\left(\varepsilon_{x x}^{n l}\right)^{8}+\left(\gamma^{2}-4 \gamma+3\right)\left(\varepsilon_{c}^{n l}\right)^{2}\left(\varepsilon_{x x}^{n l}\right)^{7}+ \\
& {\left[\left(2 \gamma^{2}-3\right) \alpha C_{x}-\gamma^{3} \alpha C_{z}-\left(\gamma^{2}-2 \gamma+1\right)\left(\varepsilon_{c}^{n l}\right)^{3}\right]\left(\varepsilon_{x x}^{n l}\right)^{6}+2\left(\gamma^{3}-2 \gamma^{2}-2 \gamma+3\right) \alpha C_{x} \varepsilon_{c}^{n l}\left(\varepsilon_{x x}^{n l}\right)^{5}+} \\
& \left(-2 \gamma^{3}+\gamma^{2}+4 \gamma-3\right) \alpha C_{x}\left(\varepsilon_{c}^{n l}\right)^{2}\left(\varepsilon_{x x}^{n l}\right)^{4}+\left(\gamma^{4}-4 \gamma^{2}+3\right) \alpha^{2} C_{x}^{2}\left(\varepsilon_{x x}^{n l}\right)^{3}+ \\
& \quad\left(-\gamma^{4}-2 \gamma^{3}+4 \gamma^{2}+2 \gamma-3\right) \alpha^{2} C_{x}^{2} \varepsilon_{c}^{n l}\left(\varepsilon_{x x}^{n l}\right)^{2}+\left(-\gamma^{4}+2 \gamma^{2}-1\right) \alpha^{3} C_{x}^{3}=0
\end{aligned}
$$

A permissividade $\varepsilon_{z z}^{n l}$ pode, agora, ser calculada utilizando (3.24) e (3.25), ou seja:

$$
\varepsilon_{z z}^{n l}=\frac{1}{\gamma}\left[\varepsilon_{x x}^{n l}+(\gamma-1) \varepsilon_{c}^{n l}+\alpha\left(\gamma^{2}-1\right) \frac{C_{x}}{\left(\varepsilon_{x x}^{n l}\right)^{2}}\right] .
$$


As raízes de (3.28) são calculadas numericamente por meio de rotina que utiliza o algoritmo de Jenkins-Traub de três estágios [56], do qual a raiz procurada deve ser tomada de tal modo que $\varepsilon_{x x}^{n l}$ e $\varepsilon_{z z}^{n l}$ sejam reais e o mais próximas possível da permissividade estática $\varepsilon_{c}^{n l}$. Note que para o caso particular $\gamma=1, \varepsilon_{x x}^{n l}=\varepsilon_{z z}^{n l}$, e (3.28) é reduzida à seguinte equação cúbica, cuja raiz pode ser obtida explicitamente:

$$
\left(\varepsilon_{x x}^{n l}\right)^{3}-\varepsilon_{c}^{n l}\left(\varepsilon_{x x}^{n l}\right)^{2}-\alpha\left(C_{x}+C_{z}\right)=0 .
$$

O procedimento descrito acima visa calcular as permissividades na região não-linear para $z=(m+1) \delta z$. Portanto, observando (3.26) e (3.27), conclui-se que

$$
\begin{gathered}
C_{x}=\left.C_{x}\right|_{i} ^{m+1}=\frac{1}{\omega^{2} \varepsilon_{0}^{2}}\left|\kappa \Psi_{i}^{m+1}+j \frac{\left(\Psi_{i}^{m+1}\right)^{2} / \Psi_{i}^{m}-\Psi_{i}^{m}}{2 \delta z}\right|^{2}, \\
C_{z}=\left.C_{z}\right|_{i} ^{m+1}=\frac{1}{\omega^{2} \varepsilon_{0}^{2}}\left|v_{i} \frac{\Psi_{i+1}^{m+1}-\Psi_{i-1}^{m+1}}{2 \delta x}\right|^{2},
\end{gathered}
$$

observando o fato de que foi necessário embutir a seguinte aproximação em (3.31):

$$
\Psi_{i}^{m+2} \approx \frac{\left(\Psi_{i}^{m+1}\right)^{2}}{\Psi_{i}^{m}}
$$

Portanto, o referido procedimento iterativo envolve as equações (3.28) e (3.29), que serão usadas para atualizar a permissividade elétrica na região não-linear para o passo longitudinal atual. Esta distribuição de permissividade vai ser utilizada para recalcular a distribuição transversal de campo no passo longitudinal atual. A atualização da permissividade elétrica e a sua aplicação na estimativa de uma distribuição transversal de campo mais precisa serão repetidas até que o seguinte critério de convergência seja satisfeito: 


$$
\operatorname{Max}\left[\frac{\left|\Psi^{m+1}-\widetilde{\Psi}^{m+1}\right|}{\left|\Psi^{m+1}\right|}\right]<\delta_{t o l},
$$

na qual $\widetilde{\Psi}^{m+1}$ é a estimativa anterior para a distribuição transversal de campo.

\subsection{Condições de contorno}

\subsubsection{Camada Perfeitamente Casada ou PML (Perfectly Matched Layer)}

Consiste em uma condição de contorno absorvente baseada na incorporação de perfis de condutividade elétrica $\left(\sigma_{\mathrm{E}}\right)$ e magnética $\left(\sigma_{\mathrm{H}}\right)$ nos materiais que sofreram truncamento numérico. Estas condutividades estão relacionadas pela condição $\sigma_{H} / \mu$ $=\sigma_{\mathrm{E}} / \varepsilon$, de forma a apresentar a mesma impedância característica do material original. Isto pode ser feito uma vez que a PML nada mais é que a terminação casada deste último, cuja função é dissipar a energia transmitida para a mesma.

Sabendo que a implementação desta técnica exige a modificação das equações de Maxwell, sua aplicação, baseada na técnica de transformação de coordenadas (stretched coordinates) [10]-[12], já foi exposta previamente e em paralelo com o desenvolvimento do formalismo FD-BPM. Resta somente fornecer maiores detalhes sobre perfil de condutividade, o qual apresenta a seguinte variação:

$$
\sigma_{H}(\tau)=\sigma_{H \max }\left(\frac{\tau}{\delta x N p m l}\right)^{q}
$$

sendo $\mathrm{Npml}$ o número de células da camada PML na direção normal à interface com o meio adjacente. O perfil de condutividade é crescente das interfaces $(\tau=0)$ para os limites espaciais transversais $(\tau=\delta x N p m l)$. Um valor coerente para $\sigma_{H \max }$ pode ser estimado a partir do coeficiente de reflexão teórico para incidência normal [11]: 


$$
R(\pi / 2)=\exp \left[-2 \frac{k_{0} n \delta x N p m l \sigma_{H \max }}{\omega \mu_{0}(q+1)}\right],
$$

na qual $n$ é o índice de refração da PML.

\subsubsection{Condição de Contorno Transparente (TBC)}

Esta técnica baseia-se na imposição de uma continuidade virtual aos campos que se propagam através dos limites transversais de modo que apenas um fluxo de energia de valor negativo (ondas que deixam o domínio espacial) seja permitido. Esta condição de transparência é eficiente em muitos casos e modificações nas equações de Maxwell ou na equação de onda não são requeridas. Como exemplo, o campo na extremidade esquerda da janela computacional é atualizado da seguinte forma:

$$
\widetilde{\psi}_{0}^{m+1}=\psi_{1}^{m+1} \exp \left(j \widetilde{k}_{x}^{\prime} \delta x\right),
$$

na qual $\tilde{k}_{x}^{\prime}=-\frac{j}{\delta x} \ln \left(\frac{\psi_{1}^{m}}{\psi_{2}^{m}}\right)$.

Note que $\mathfrak{R}\left\{\tilde{k}_{x}^{\prime}\right\}$ deve ser negativo a fim de caracterizar apenas ondas transmitidas para fora da janela computacional. Dessa forma, se $\mathfrak{R}\left\{\widetilde{k}_{x}^{\prime}\right\}>0$ então $\widetilde{k}_{x}^{\prime}=\mathfrak{J}\left\{\widetilde{k}_{x}^{\prime}\right\}$ (nesta relação, $\mathfrak{R}$ e $\mathfrak{I}$ referem-se à parte real e imaginária de um argumento complexo, respectivamente). 


\section{MÉTODO FD-BPM TRIDIMENSIONAL VETORIAL BASEADO NA TÉCNICA ADI}

Esta seção mostra o procedimento proposto na criação de um algoritmo que possibilita a consideração de meios isotrópicos, anisotrópicos e não-lineares em dispositivos baseados em guias de ondas ópticos tridimensionais. Este método combina pela primeira vez a técnica implícita de direções alternantes (ADI) e leapfrog para a simulação vetorial eficiente de estruturas com geometrias diversas e formadas por estes materiais. Contudo é feita uma restrição com relação a anisotropia dielétrica com o objetivo de simplificar o formalismo. A formulação inclui a condição de contorno transparente TBC [32] para o truncamento dos limites espaciais transversais.

\subsection{Expansão do formalismo FD-BPM}

A Equação de Helmholtz é dada por

$$
k_{0}^{2} \overline{\bar{\varepsilon}} \overline{\mathrm{E}}+\nabla^{2} \bar{E}=\nabla(\nabla \cdot \bar{E}),
$$

na qual o tensor permissividade elétrica é escrito de forma a simplificar o acoplamento entre as componentes transversais de campo elétrico e a componente longitudinal:

$$
\overline{\bar{\varepsilon}}=\left(\begin{array}{ccc}
\varepsilon_{x x} & \varepsilon_{x y} & 0 \\
\varepsilon_{y x} & \varepsilon_{y y} & 0 \\
0 & 0 & \varepsilon_{z z}
\end{array}\right)
$$

Deste modo, conforme será demonstrado em breve, é possível eliminar a componente $E_{z}$, evitando que esta seja considerada diretamente no processo 
recursivo. A componente $E_{z}$ será eliminada através da aplicação da Lei de Gauss, o que garante, adicionalmente, que o divergente do vetor densidade de fluxo elétrico é nulo para meios livres de cargas, evitando o surgimento de modos espúrios. Portanto, o tratamento numérico envolverá apenas as equações para as componentes transversais, as quais serão interligadas pela aplicação de um esquema de discretização longitudinal leapfrog, conforme será visto adiante. Assim a equação para uma componente transversal conterá termos envolvendo a outra. Expandindo (4.1), obtém-se

$$
\begin{gathered}
k_{0}^{2}\left(\varepsilon_{x x} E_{x}+\varepsilon_{x y} E_{y}\right)+\frac{\partial^{2} E_{x}}{\partial y^{2}}+\frac{\partial^{2} E_{x}}{\partial z^{2}}=\frac{\partial}{\partial x}\left(\frac{\partial E_{y}}{\partial y}+\frac{\partial E_{z}}{\partial z}\right) \\
k_{0}^{2}\left(\varepsilon_{y x} E_{x}+\varepsilon_{y y} E_{y}\right)+\frac{\partial^{2} E_{y}}{\partial x^{2}}+\frac{\partial^{2} E_{y}}{\partial z^{2}}=\frac{\partial}{\partial y}\left(\frac{\partial E_{x}}{\partial x}+\frac{\partial E_{z}}{\partial z}\right) \\
k_{0}^{2}\left(\varepsilon_{z z} E_{z}\right)+\frac{\partial^{2} E_{z}}{\partial x^{2}}+\frac{\partial^{2} E_{z}}{\partial y^{2}}=\frac{\partial}{\partial z}\left(\frac{\partial E_{x}}{\partial x}+\frac{\partial E_{y}}{\partial y}\right)
\end{gathered}
$$

A partir da lei de Gauss, $\nabla \cdot \bar{D}=0$ (para neutralidade de cargas), e considerando que a geometria varia lentamente ao longo da direção de propagação $\left(\partial \varepsilon_{z z} / \partial z \approx 0\right)$ [42], obtém-se

$$
\frac{\partial E_{z}}{\partial z}=-\frac{1}{\varepsilon_{z z}} \frac{\partial}{\partial x}\left(\varepsilon_{x x} E_{x}\right)-\frac{1}{\varepsilon_{z z}} \frac{\partial}{\partial x}\left(\varepsilon_{x y} E_{y}\right)-\frac{1}{\varepsilon_{z z}} \frac{\partial}{\partial y}\left(\varepsilon_{y x} E_{x}\right)-\frac{1}{\varepsilon_{z z}} \frac{\partial}{\partial y}\left(\varepsilon_{y y} E_{y}\right)
$$

Substituindo (4.6) em (4.3) e (4.4), obtém-se, respectivamente

$$
\begin{aligned}
& k_{0}^{2} \varepsilon_{x x} E_{x}+\frac{\partial}{\partial x}\left[\frac{1}{\varepsilon_{z z}} \frac{\partial}{\partial x}\left(\varepsilon_{x x} E_{x}\right)\right]+\frac{\partial^{2} E_{x}}{\partial y^{2}}+\frac{\partial}{\partial x}\left[\frac{1}{\varepsilon_{z z}} \frac{\partial}{\partial y}\left(\varepsilon_{y x} E_{x}\right)\right]+\frac{\partial^{2} E_{x}}{\partial^{2} z}= \\
& \frac{\partial^{2} E_{y}}{\partial x \partial y}-k_{0}^{2} \varepsilon_{x y} E_{y}-\frac{\partial}{\partial x}\left[\frac{1}{\varepsilon_{z z}} \frac{\partial}{\partial x}\left(\varepsilon_{x y} E_{y}\right)\right]-\frac{\partial}{\partial x}\left[\frac{1}{\varepsilon_{z z}} \frac{\partial}{\partial y}\left(\varepsilon_{y y} E_{y}\right)\right] \\
& k_{0}^{2} \varepsilon_{y y} E_{y}+\frac{\partial^{2} E_{y}}{\partial x^{2}}+\frac{\partial}{\partial y}\left[\frac{1}{\varepsilon_{z z}} \frac{\partial}{\partial y}\left(\varepsilon_{y y} E_{y}\right)\right]+\frac{\partial}{\partial y}\left[\frac{1}{\varepsilon_{z z}} \frac{\partial}{\partial x}\left(\varepsilon_{x y} E_{y}\right)\right]+\frac{\partial^{2} E_{y}}{\partial^{2} z}= \\
& -k_{0}^{2} \varepsilon_{y x} E_{x}+\frac{\partial^{2} E_{x}}{\partial x \partial y}-\frac{\partial}{\partial y}\left[\frac{1}{\varepsilon_{z z}} \frac{\partial}{\partial x}\left(\varepsilon_{x x} E_{x}\right)\right]-\frac{\partial}{\partial y}\left[\frac{1}{\varepsilon_{z z}} \frac{\partial}{\partial y}\left(\varepsilon_{y x} E_{x}\right)\right]
\end{aligned}
$$


Observe que a partir deste ponto a equação (4.5) (componente $E_{z}$ ) pode ser descartada do formalismo. Aplicando a aproximação por envoltória de variação lenta (SVEA) nas componentes de campo elétrico, supõe-se que

$$
\bar{E}=\bar{\Psi} \exp (-j \kappa z), \quad \text { na qual } \kappa=k_{0} n_{0},
$$

tal que $n_{0}$ é um índice efetivo de referência.

Substituindo (4.9) em (4.7)-(4.8) e considerando $\left|\frac{\partial^{2} \Psi_{u}}{\partial z^{2}}\right|<<2 \kappa\left|\frac{\partial \Psi_{u}}{\partial z}\right|$, resulta em

$$
\begin{aligned}
& k_{0}^{2}\left(\varepsilon_{x x}-n_{0}^{2}\right) \Psi_{x}+\frac{\partial}{\partial x}\left[\frac{1}{\varepsilon_{z z}} \frac{\partial}{\partial x}\left(\varepsilon_{x x} \Psi_{x}\right)\right]+\frac{\partial^{2} \Psi_{x}}{\partial y^{2}}+\frac{\partial}{\partial x}\left[\frac{1}{\varepsilon_{z z}} \frac{\partial}{\partial y}\left(\varepsilon_{y x} \Psi_{x}\right)\right]-2 j \kappa \frac{\partial \Psi_{x}}{\partial z}= \\
& \frac{\partial^{2} \Psi_{y}}{\partial x \partial y}-k_{0}^{2} \varepsilon_{x y} \Psi_{y}-\frac{\partial}{\partial x}\left[\frac{1}{\varepsilon_{z z}} \frac{\partial}{\partial x}\left(\varepsilon_{x y} \Psi_{y}\right)\right]-\frac{\partial}{\partial x}\left[\frac{1}{\varepsilon_{z z}} \frac{\partial}{\partial y}\left(\varepsilon_{y y} \Psi_{y}\right)\right] \\
& k_{0}^{2}\left(\varepsilon_{y y}-n_{0}^{2}\right) \Psi_{y}+\frac{\partial^{2} \Psi_{y}}{\partial x^{2}}+\frac{\partial}{\partial y}\left[\frac{1}{\varepsilon_{z z}} \frac{\partial}{\partial y}\left(\varepsilon_{y y} \Psi_{y}\right)\right]+\frac{\partial}{\partial y}\left[\frac{1}{\varepsilon_{z z}} \frac{\partial}{\partial x}\left(\varepsilon_{x y} \Psi_{y}\right)\right]-2 j \kappa \frac{\partial \Psi_{y}}{\partial z}= \\
& -k_{0}^{2} \varepsilon_{y x} \Psi_{x}+\frac{\partial^{2} \Psi_{x}}{\partial x \partial y}-\frac{\partial}{\partial y}\left[\frac{1}{\varepsilon_{z z}} \frac{\partial}{\partial x}\left(\varepsilon_{x x} \Psi_{x}\right)\right]-\frac{\partial}{\partial y}\left[\frac{1}{\varepsilon_{z z}} \frac{\partial}{\partial y}\left(\varepsilon_{y x} \Psi_{x}\right)\right]
\end{aligned}
$$

A partir de (4.10) e (4.11), obtém-se as equações FD-BPM para resolver as componentes $\psi_{\mathrm{x}}$ e $\psi_{\mathrm{y}}$, respectivamente, considerando-se o acoplamento entre estas. Discretizando (4.10) ao longo do eixo longitudinal, de forma que seja adotado um esquema leapfrog entre as componentes $\Psi_{\mathrm{x}}$ e $\Psi_{\mathrm{y}}$, resulta em

$$
\begin{aligned}
& {\left[1-\frac{\delta z}{4 j \kappa}\left(F_{x}+D_{y}+v_{x x}\right)\right] \Psi_{x}^{K+\frac{1}{2}}=} \\
& {\left[1+\frac{\delta z}{4 j \kappa}\left(F_{x}+D_{y}+v_{x x}\right)\right] \Psi_{x}^{K-\frac{1}{2}}+\frac{\delta z}{2 j \kappa}\left(T_{x y}^{K-\frac{1}{2}}-V_{x y}^{K}\right)}
\end{aligned}
$$

na qual

$$
\begin{gathered}
F_{x} \Psi_{x}=\frac{\partial}{\partial x}\left[\frac{1}{\varepsilon_{z z}} \frac{\partial}{\partial x}\left(\varepsilon_{x x} \Psi_{x}\right)\right] \\
D_{y} \Psi_{x}=\frac{\partial^{2} \Psi_{x}}{\partial y^{2}}
\end{gathered}
$$




$$
\begin{gathered}
v_{x x}=k_{0}^{2}\left(\varepsilon_{x x}-n_{0}^{2}\right) \\
V_{x y}^{K}=\frac{\partial^{2} \Psi_{y}^{K}}{\partial x \partial y}-k_{0}^{2} \varepsilon_{x y} \Psi_{y}^{K}-\frac{\partial}{\partial x}\left[\frac{1}{\varepsilon_{z z}} \frac{\partial}{\partial x}\left(\varepsilon_{x y} \Psi_{y}^{K}\right)\right]-\frac{\partial}{\partial x}\left[\frac{1}{\varepsilon_{z z}} \frac{\partial}{\partial y}\left(\varepsilon_{y y} \Psi_{y}^{K}\right)\right] \\
T_{x y}^{K-\frac{1}{2}}=\frac{\partial}{\partial x}\left[\frac{1}{\varepsilon_{z z}} \frac{\partial}{\partial y}\left(\varepsilon_{y x} \Psi_{x}^{K-\frac{1}{2}}\right)\right]
\end{gathered}
$$

Com a adoção de um esquema leapfrog não é necessário aplicar o esquema Crank-Nicolson $(\mathrm{CN})$ nos termos que envolve $\Psi_{\mathrm{y}}$ ao se extrair a equação FD-BPM para $\psi_{\mathrm{x}}$, e vice-versa. Portanto, quando se resolve uma componente transversal, termos envolvendo a outra componente transversal já são totalmente conhecidos.

Para possibilitar a aplicação da técnica ADI, (4.12) é fatorada:

$$
\begin{aligned}
& {\left[1-\frac{\delta z}{4 j \kappa}\left(F_{x}+\frac{v_{1}}{2}\right)\right]\left[1-\frac{\delta z}{4 j \kappa}\left(D_{y}+\frac{v_{x x}}{2}\right)\right] \Psi_{x}^{K+\frac{1}{2}}=} \\
& {\left[1+\frac{\delta z}{4 j \kappa}\left(F_{x}+\frac{v_{1}}{2}\right)\right]\left[1+\frac{\delta z}{4 j \kappa}\left(D_{y}+\frac{v_{x x}}{2}\right)\right] \Psi_{x}^{K-\frac{1}{2}}+\frac{\delta z}{2 j \kappa}\left(T_{x y}^{K-\frac{1}{2}}-V_{x y}^{K}\right)}
\end{aligned}
$$

O erro de aproximação introduzido na obtenção de (4.18) é proporcional a $\mathrm{O}\left(\delta z^{2}\right)$, o qual é da mesma ordem do erro de truncamento. Conseqüentemente, a precisão numérica global não é afetada. Realizando decomposição do tipo DouglasRachford [48] em (4.18), obtém-se

$$
\begin{aligned}
& {\left[1-\frac{\delta z}{4 j \kappa}\left(F_{x}+\frac{v_{x x}}{2}\right)\right] \Theta_{x}^{K+\frac{1}{2}}=} \\
& {\left[1+\frac{\delta z}{4 j \kappa}\left(F_{x}+\frac{v_{x x}}{2}\right)+\frac{\delta z}{2 j \kappa}\left(D_{y}+\frac{v_{x x}}{2}\right)\right] \Psi_{x}^{K-\frac{1}{2}}+\frac{\delta z}{2 j \kappa}\left(T_{x y}^{K-\frac{1}{2}}-V_{x y}^{K}\right)} \\
& {\left[1-\frac{\delta z}{4 j \kappa}\left(D_{y}+\frac{v_{x x}}{2}\right)\right] \Psi_{x}^{K+\frac{1}{2}}=\Theta_{x}^{K+\frac{1}{2}}-\frac{\delta z}{4 j \kappa}\left(D_{y}+\frac{v_{x x}}{2}\right) \Psi_{x}^{K-\frac{1}{2}}}
\end{aligned}
$$

As equações (4.19) e (4.20) referem-se ao primeiro e segundo passos do ADI, respectivamente, para o cálculo recursivo da componente $\Psi_{x}$. A variável $\Theta_{x}^{K+1}$ é uma 
distribuição transversal de campo auxiliar, a qual é calculada no primeiro passo do ADI, sendo posteriormente transmitida ao segundo, para o cálculo do campo realmente almejado, $\Psi_{x}^{K+\frac{1}{2}}$.

Em relação ao cálculo componente $\Psi_{y}$, o procedimento tem inicio a partir da discretização de (4.11) ao longo do eixo longitudinal, resultando em

$$
\begin{aligned}
& {\left[1-\frac{\delta z}{4 j \kappa}\left(D_{x}+F_{y}+v_{y y}\right)\right] \Psi_{y}^{K+1}=} \\
& {\left[1+\frac{\delta z}{4 j \kappa}\left(D_{x}+F_{y}+v_{y y}\right)\right] \Psi_{y}^{K}+\frac{\delta z}{2 j \kappa}\left(T_{y x}^{K}-V_{y x}^{K+\frac{1}{2}}\right)}
\end{aligned}
$$

na qual

$$
\begin{aligned}
& D_{x} \Psi_{y}=\frac{\partial^{2} \Psi_{y}}{\partial x^{2}} \\
& F_{y} \Psi_{y}=\frac{\partial}{\partial y}\left[\frac{1}{\varepsilon_{z z}} \frac{\partial}{\partial y}\left(\varepsilon_{y y} \Psi_{y}\right)\right] \\
& v_{y y}=k_{0}^{2}\left(\varepsilon_{y y}-n_{0}^{2}\right) \\
& V_{y x}^{K+\frac{1}{2}}=\frac{\partial^{2} \Psi_{x}^{K+\frac{1}{2}}}{\partial x \partial y}-k_{0}^{2} \varepsilon_{y x} \Psi_{x}^{K+\frac{1}{2}}-\frac{\partial}{\partial y}\left[\frac{1}{\varepsilon_{z z}} \frac{\partial}{\partial x}\left(\varepsilon_{x x} \Psi_{x}^{K+\frac{1}{2}}\right)\right]-\frac{\partial}{\partial y}\left[\frac{1}{\varepsilon_{z z}} \frac{\partial}{\partial y}\left(\varepsilon_{y x} \Psi_{x}^{K+\frac{1}{2}}\right)\right] \\
& T_{y x}^{K}=\frac{\partial}{\partial y}\left[\frac{1}{\varepsilon_{z z}} \frac{\partial}{\partial x}\left(\varepsilon_{x y} \Psi_{y}^{K}\right)\right]
\end{aligned}
$$

Para possibilitar a aplicação da técnica ADI, a equação (4.21) é fatorada:

$$
\begin{aligned}
& {\left[1-\frac{\delta z}{4 j \kappa}\left(D_{x}+\frac{v_{y y}}{2}\right)\right]\left[1-\frac{\delta z}{4 j \kappa}\left(F_{y}+\frac{v_{y y}}{2}\right)\right] \Psi_{y}^{K+1}=} \\
& {\left[1+\frac{\delta z}{4 j \kappa}\left(D_{x}+\frac{v_{y y}}{2}\right)\right]\left[1+\frac{\delta z}{4 j \kappa}\left(F_{y}+\frac{v_{y y}}{2}\right)\right] \Psi_{y}^{K}+\frac{\delta z}{2 j \kappa}\left(T_{y x}^{K}-V_{y x}^{K+\frac{1}{2}}\right)}
\end{aligned}
$$

Realizando decomposição do tipo Douglas-Rachford [48] em (4.27), obtém-se 


$$
\begin{aligned}
& {\left[1-\frac{\delta z}{4 j \kappa}\left(D_{x}+\frac{v_{y y}}{2}\right)\right] \Theta_{y}^{K+1}=} \\
& {\left[1+\frac{\delta z}{4 j \kappa}\left(D_{x}+\frac{v_{y y}}{2}\right)+\frac{\delta z}{2 j \kappa}\left(F_{y}+\frac{v_{y y}}{2}\right)\right] \Psi_{y}^{K}+\frac{\delta z}{2 j \kappa}\left(T_{y x}^{K}-V_{y x}^{K+\frac{1}{2}}\right)} \\
& {\left[1-\frac{\delta z}{4 j \kappa}\left(F_{y}+\frac{v_{y y}}{2}\right)\right] \Psi_{y}^{K+1}=\Theta_{y}^{K+1}-\frac{\delta z}{4 j \kappa}\left(F_{y}+\frac{v_{y y}}{2}\right) \Psi_{y}^{K}}
\end{aligned}
$$

Portanto, foram realizadas as discretizações longitudinais das equações relativas ao cálculo das componentes transversais, obtendo-se (4.19)-(4.20) para $\Psi_{x} \mathrm{e}$ (4.28)-(4.29) para $\Psi_{y}$. O próximo passo é a discretização transversal dos termos envolvidos nestas equações, com a finalidade de obter-se as equações FD-BPM finais, conforme desenvolvido a seguir.

\subsubsection{FD-BPM para o cálculo da componente $x$ do vetor campo elétrico}

Nesta seção é concluída a expansão em diferenças finitas para a obtenção de $\Psi_{x}^{K+\frac{1}{2}}$. Discretizando (4.13) - (4.17) nas direções transversais, obtém-se

$$
\begin{aligned}
& F_{x} \Psi_{x(I, J)}=\frac{2}{\delta x^{2}}\left(\frac{1}{\varepsilon_{z z(I+1, J)}+\varepsilon_{z z(I, J)}}\right) \varepsilon_{x x(I+1, J)} \Psi_{x(I+1, J)}- \\
& \frac{2}{\delta x^{2}}\left(\frac{1}{\varepsilon_{z z(I+1, J)}+\varepsilon_{z z(I, J)}}+\frac{1}{\varepsilon_{z z(I, J)}+\varepsilon_{z z(I-1, J)}}\right) \varepsilon_{x x(I, J)} \Psi_{x(I, J)}+ \\
& \frac{2}{\delta x^{2}}\left(\frac{1}{\varepsilon_{z z(I, J)}+\varepsilon_{z z(I-1, J)}}\right) \varepsilon_{x x(I-1, J)} \Psi_{x(I-1, J)} \\
& D_{y} \Psi_{x(I, J)}=\frac{1}{\delta y^{2}} \Psi_{x(I, J+1)}-\frac{2}{\delta y^{2}} \Psi_{x(I, J)}+\frac{1}{\delta y^{2}} \Psi_{x(I, J-1)} \\
& v_{x x(I, J)}=k_{0}^{2}\left(\varepsilon_{x x(I, J)}-n_{0}^{2}\right)
\end{aligned}
$$




$$
\begin{aligned}
& V_{x y(I, J)}^{K}=\frac{\Psi_{y(I+1, J+1)}^{K}-\Psi_{y(I-1, J+1)}^{K}-\Psi_{y(I+1, J-1)}^{K}+\Psi_{y(I-1, J-1)}^{K}}{4 \delta x \delta y}-k_{0}^{2} \varepsilon_{x y(I, J)} \Psi_{y(I, J)}^{K} \\
& -\frac{2}{\delta x^{2}}\left(\frac{1}{\varepsilon_{z z(I+1, J)}+\varepsilon_{z z(I, J)}}\right) \varepsilon_{x y(I+1, J)} \Psi_{y(I+1, J)}^{K}+ \\
& \frac{2}{\delta x^{2}}\left(\frac{1}{\varepsilon_{z z(I+1, J)}+\varepsilon_{z z(I, J)}}+\frac{1}{\varepsilon_{z z(I, J)}+\varepsilon_{z z(I-1, J)}}\right) \varepsilon_{x y(I, J)} \Psi_{y(I, J)}^{K}- \\
& \frac{2}{\delta x^{2}}\left(\frac{1}{\varepsilon_{z z(I, J)}+\varepsilon_{z z(I-1, J)}}\right) \varepsilon_{x y(I-1, J)} \Psi_{y(I-1, J)}^{K}- \\
& \frac{1}{4 \delta x \delta y}\left[\frac{1}{\varepsilon_{z z(I+1, J)}}\left(\varepsilon_{y y(I+1, J+1)} \Psi_{y(I+1, J+1)}^{K}-\varepsilon_{y y(I+1, J-1)} \Psi_{y(I+1, J-1)}^{K}\right)-\right. \\
& \left.\frac{1}{\varepsilon_{z z(I-1, J)}}\left(\varepsilon_{y y(I-1, J+1)} \Psi_{y(I-1, J+1)}^{K}-\varepsilon_{y y(I-1, J-1)} \Psi_{y(I-1, J-1)}^{K}\right)\right] \\
& T_{x y(I, J)}^{K-\frac{1}{2}}=\frac{1}{4 \delta x \delta y}\left[\frac{1}{\varepsilon_{z z(I+1, J)}}\left(\varepsilon_{y x(I+1, J+1)} \Psi_{x(I+1, J+1)}^{K-\frac{1}{2}}-\varepsilon_{y x(I+1, J-1)} \Psi_{x(I+1, J-1)}^{K-\frac{1}{2}}\right)-\right. \\
& \left.\frac{1}{\varepsilon_{z z(I-1, J)}}\left(\varepsilon_{y x(I-1, J+1)} \Psi_{x(I-1, J+1)}^{K-\frac{1}{2}}-\varepsilon_{y x(I-1, J-1)} \Psi_{x(I-1, J-1)}^{K-\frac{1}{2}}\right)\right]
\end{aligned}
$$

Aplicando o operador (4.30) em $\Theta_{x}^{K+\frac{1}{2}}$ e substituindo o resultado em (4.19), obtém-se

$$
\begin{aligned}
& \phi_{1(I-1, J)} \Theta_{x(I-1, J)}^{K+\frac{1}{2}}+\theta_{1(I, J)} \Theta_{x(I, J)}^{K+\frac{1}{2}}+\phi_{1(I+1, J)} \Theta_{x(I+1, J)}^{K+\frac{1}{2}}= \\
& {\left[1+\frac{\delta z}{4 j \kappa}\left(F_{x}+\frac{v_{x x(I, J)}}{2}\right)+\frac{\delta z}{2 j \kappa}\left(D_{y}+\frac{\left.v_{x x(I, J)}\right)}{2}\right)\right] \Psi_{x(I, J)}^{K-\frac{1}{2}}+} \\
& \frac{\delta z}{2 j \kappa}\left(T_{x y(I, J)}^{K-\frac{1}{2}}-V_{x y(I, J)}^{K}\right)
\end{aligned}
$$

na qual

$$
\begin{aligned}
& \phi_{(\text {Iref }, J)}=-\frac{\delta z}{2 j \kappa} \frac{1}{\delta x^{2}}\left(\frac{1}{\varepsilon_{z z(I, J)}+\varepsilon_{z z(I r e f, J)}}\right) \varepsilon_{x x(\text { Iref }, J)}^{K+\frac{1}{2}} \\
& \theta_{(I, J)}=1+\frac{\delta z}{8 j \kappa}\left[\frac{4}{\delta x^{2}}\left(\frac{1}{\varepsilon_{z z(I+1, J)}+\varepsilon_{z z(I, J)}}+\frac{1}{\varepsilon_{z z(I, J)}+\varepsilon_{z z(I-1, J)}}\right) \varepsilon_{x x(I, J)}^{K+\frac{1}{2}}-v_{x x(I, J)}\right]
\end{aligned}
$$


Aplicando o operador (4.31) em $\Psi_{x}^{K+\frac{1}{2}}$ e substituindo o resultado em (4.20), obtém-se

$$
\phi_{2} \Psi_{x(I, J-1)}^{K+\frac{1}{2}}+\theta_{2(I, J)} \Psi_{x(I, J)}^{K+\frac{1}{2}}+\phi_{2} \Psi_{x(I, J+1)}^{K+\frac{1}{2}}=\Theta_{x(I, J)}^{K+\frac{1}{2}}-\frac{\delta z}{4 j \kappa}\left(D_{y}+\frac{v_{x x}}{2}\right) \Psi_{x(I, J)}^{K-\frac{1}{2}}
$$

sendo

$\phi_{2}=-\frac{\delta z}{4 j \kappa \delta y^{2}}$

$\theta_{2(I, J)}=1+\frac{\delta z}{8 j \kappa}\left(\frac{4}{\delta y^{2}}-v_{x x(I, J)}\right)$

Portanto, em uma determinada posição longitudinal, a obtenção da componente $\Psi_{x}^{K+\frac{1}{2}}$ é completada após a execução de dois passos. O primeiro passo do ADI é realizado ao se calcular, para cada valor de $J$, a equação tridiagonal (4.35), obtendose dessa forma a matriz de campo auxiliar $\Theta_{x}^{K+\frac{1}{2}}$. O segundo passo é realizado pelo cálculo da equação tridiagonal (4.36), para cada valor de $I$.

Resumindo, o primeiro passo resolve implicitamente linhas, enquanto o segundo passo resolve implicitamente colunas, visando à obtenção da distribuição transversal de campo desejada.

Finalmente, para possibilitar a consideração de meios não-lineares, um procedimento iterativo foi incluído, de modo que a convergência entre o campo elétrico e a permissividade dielétrica seja atingida. Para o exemplo de um meio do tipo Kerr com saturação, a permissividade dielétrica apresenta a seguinte dependência com o campo elétrico:

$$
\varepsilon=\varepsilon_{L}+\alpha_{2}|E|^{2}-\alpha_{4}|E|^{4}
$$

tal que

$$
|E|=\sqrt{\left|\Psi_{x}^{K+\frac{1}{2}}\right|^{2}+\left|\Psi_{y}^{K-1}\right|^{2}+\left|E_{z}^{K-1}\right|^{2}},
$$

na qual a componente ao longo de $z\left(E_{z}^{K-1}\right)$ não está representada na forma de envoltória de variação lenta, pois é calculada aparte do processo iterativo, por intermédio da lei de Gauss, (4.6). 
Mais detalhes sobre o modelo não-linear da Eq. (4.37) são expostos no Apêndice D.

Para que meios não-lineares sejam considerados com maior precisão no formalismo, um procedimento iterativo foi incluído para possibilitar a convergência entre a permissividade dielétrica e o campo elétrico. Portanto, (4.37) e $\Psi_{x}^{K+\frac{1}{2}}$ serão calculados iterativamente até que a seguinte condição seja satisfeita:

$$
\operatorname{Max}\left[\frac{\left|\Psi_{x}^{K+\frac{1}{2}}-\widetilde{\Psi}_{x}^{K+\frac{1}{2}}\right|}{\left|\Psi_{x}^{K+\frac{1}{2}}\right|}\right]<\delta_{t o l},
$$

na qual $\widetilde{\Psi}_{x}^{K+\frac{1}{2}}$ é a distribuição transversal calculada na iteração anterior.

\subsubsection{FD-BPM para a componente $y$ do vetor campo elétrico}

Nesta seção é concluída a expansão em diferenças finitas para a obtenção de $\Psi_{y}^{K+1}$. Discretizando (4.22) - (4.26) nas direções transversais, obtém-se

$D_{x} \Psi_{y(I, J)}=\frac{1}{\delta x^{2}} \Psi_{y(I+1, J)}-\frac{2}{\delta x^{2}} \Psi_{y(I, J)}+\frac{1}{\delta x^{2}} \Psi_{y(I-1, J)}$

$F_{y} \Psi_{y(I, J)}=\frac{2}{\delta y^{2}}\left(\frac{1}{\varepsilon_{z z(I, J+1)}+\varepsilon_{z z(I, J)}}\right) \varepsilon_{y y(I, J+1)} \Psi_{y(I, J+1)}-$

$\frac{2}{\delta y^{2}}\left(\frac{1}{\varepsilon_{z z(I, J+1)}+\varepsilon_{z z(I, J)}}+\frac{1}{\varepsilon_{z z(I, J)}+\varepsilon_{z z(I, J-1)}}\right) \varepsilon_{y y(I, J)} \Psi_{y(I, J)}+$

$\frac{2}{\delta y^{2}}\left(\frac{1}{\varepsilon_{z z(I, J)}+\varepsilon_{z z(I, J-1)}}\right) \varepsilon_{y y(I, J-1)} \Psi_{y(I, J-1)}$

$v_{y y(I, J)}=k_{0}^{2}\left(\varepsilon_{y y(I, J)}-n_{0}^{2}\right)$ 


$$
\begin{aligned}
& V_{y x(I, J)}^{K+\frac{1}{2}}=\frac{1}{4 \delta x \delta y}\left(\Psi_{x(I+1, J+1)}^{K+\frac{1}{2}}-\Psi_{x(I-1, J+1)}^{K+\frac{1}{2}}-\Psi_{x(I+1, J-1)}^{K+\frac{1}{2}}+\Psi_{x(I-1, J-1)}^{K+\frac{1}{2}}\right)-k_{0}^{2} \varepsilon_{y x} \Psi_{x(I, J)}^{K+\frac{1}{2}}- \\
& \frac{1}{4 \delta x \delta y}\left[\frac{1}{\varepsilon_{z z(I, J+1)}}\left(\varepsilon_{x x(I+1, J+1)} \Psi_{x(I+1, J+1)}^{K+\frac{1}{2}}-\varepsilon_{x x(I-1, J+1)} \Psi_{x(I-1, J+1)}^{K+\frac{1}{2}}\right)-\right. \\
& \left.\frac{1}{\varepsilon_{z z(I, J-1)}}\left(\varepsilon_{x x(I+1, J-1)} \Psi_{x(I+1, J-1)}^{K+\frac{1}{2}}-\varepsilon_{x x(I-1, J-1)} \Psi_{x(I-1, J-1)}^{K+\frac{1}{2}}\right)\right] \\
& -\frac{2}{\delta y^{2}}\left(\frac{1}{\varepsilon_{z z(I, J+1)}+\varepsilon_{z z(I, J)}}\right) \varepsilon_{y x(I, J+1)} \Psi_{x(I, J+1)}^{K+\frac{1}{2}}+ \\
& \frac{2}{\delta y^{2}}\left(\frac{1}{\varepsilon_{z z(I, J+1)}+\varepsilon_{z z(I, J)}}+\frac{1}{\varepsilon_{z z(I, J)}+\varepsilon_{z z(I, J-1)}}\right) \varepsilon_{y x(I, J)} \Psi_{x(I, J)}^{K+\frac{1}{2}}- \\
& \frac{2}{\delta y^{2}}\left(\frac{1}{\varepsilon_{z z}(I, J)+\varepsilon_{z z}(I, J-1)}\right) \varepsilon_{y x(I, J-1)} \Psi_{x(I, J-1)}^{K+\frac{1}{2}} \\
& \left.\left.\frac{T_{y x(I, J)}^{K}=\frac{1}{4 \delta x \delta y}\left[\frac{1}{\varepsilon_{z z(I, J+1)}}\left(\varepsilon_{x y(I+1, J+1)} \Psi_{y(I+1, J+1)}^{K}-\varepsilon_{x y(I-1, J+1)} \Psi_{y(I-1, J+1)}^{K}\right)-\right.}{\frac{1}{\varepsilon_{z z(I, J-1)}}\left(\varepsilon_{x y}(I+1, J-1)\right.} \Psi_{y(I+1, J)}^{K}-\varepsilon_{x y(I-1, J-1)} \Psi_{y(I-1, J-1)}^{K}\right)\right]
\end{aligned}
$$

Aplicando o operador (4.40) em $\Theta_{y}^{K+1}$ e substituindo o resultado em (4.28), obtém-se

$$
\begin{aligned}
& \phi_{3} \Theta_{y(I-1, J)}^{K+1}+\theta_{3(I, J)} \Theta_{y(I, J)}^{K+1}+\phi_{3} \Theta_{y(I+1, J)}^{K+1}= \\
& {\left[1+\frac{\delta z}{4 j \kappa}\left(D_{x}+\frac{v_{y y(I, J)}}{2}\right)+\frac{\delta z}{2 j \kappa}\left(F_{y}+\frac{v_{y y(I, J)}}{2}\right)\right] \Psi_{y(I, J)}^{K}+\frac{\delta z}{2 j \kappa}\left(T_{y x(I, J)}^{K}-V_{y x(I, J)}^{K+\frac{1}{2}}\right)}
\end{aligned}
$$

na qual

$$
\begin{aligned}
& \phi_{3}=-\frac{\delta z}{4 j \kappa \delta x^{2}} \\
& \theta_{3(I, J)}=1+\frac{\delta z}{4 j \kappa}\left(\frac{2}{\delta x^{2}}-\frac{v_{y y(I, J)}}{2}\right)
\end{aligned}
$$

Aplicando o operador (4.41) em $\Psi_{y}^{K+1}$ e substituindo o resultado em (4.29), obtém-se 


$$
\begin{aligned}
& \phi_{4(I, J-1)} \Psi_{y(I, J-1)}^{K+1}+\theta_{4(I, J)} \Psi_{y(I, J)}^{K+1}+\phi_{4(I, J+1)} \Psi_{y(I, J+1)}^{K+1}= \\
& \Theta_{y(I, J)}^{K+1}-\frac{\delta z}{4 j \kappa}\left(F_{y}+\frac{v_{y y(I, J)}}{2}\right) \Psi_{y(I, J)}^{K},
\end{aligned}
$$

na qual

$$
\begin{aligned}
& \phi_{4(I, J r e f)}=-\frac{\delta z}{2 j \kappa} \frac{1}{\delta y^{2}}\left(\frac{1}{\varepsilon_{z z(I, J)}^{K+1}+\varepsilon_{z z(I, J r e f)}^{K+1}}\right) \varepsilon_{y y(I, J r e f)}^{K+1} \quad(\text { Jref }=J-1, J+1) \\
& \theta_{4(I, J)}=1+\frac{\delta z}{8 j \kappa}\left[\frac{4}{\delta y^{2}}\left(\frac{1}{\varepsilon_{z z(I, J+1)}^{K+1}+\varepsilon_{z z(I, J)}^{K+1}}+\frac{1}{\varepsilon_{z z(I, J)}^{K+1}+\varepsilon_{z z(I, J-1)}^{K+1}}\right) \varepsilon_{y y(I, J)}^{K+1}-v_{y y(I, J)}^{K+1}\right]
\end{aligned}
$$

Portanto, em uma determinada posição longitudinal, a obtenção da componente $\Psi_{y}^{K+1}$ é completada após a execução de dois passos. O primeiro passo do ADI é realizado ao se calcular, para cada valor de $J$, a equação tridiagonal (4.45), obtendose dessa forma a matriz de campo auxiliar $\Theta_{y}^{K+1}$. O segundo passo é realizado pelo cálculo da equação tridiagonal (4.46), para cada valor de $I$.

De forma análoga ao que foi realizado para o cálculo de $\Psi_{x}^{K+\frac{1}{2}}$ em meios nãolineares, um procedimento iterativo também é adotado para o cálculo de $\Psi_{y}^{K+1}$. Contudo, uma pequena modificação na estimativa da intensidade de campo elétrico é utilizada, considerando novamente (4.37):

$$
|E|=\sqrt{\left|\Psi_{y}^{K+1}\right|^{2}+\left|\Psi_{x}^{K+\frac{1}{2}}\right|^{2}+\left|E_{z}^{K-1}\right|^{2}}
$$

Portanto (4.37) e $\Psi_{y}^{K+1}$ serão calculados repetidamente até que a seguinte condição seja satisfeita:

$$
\operatorname{Max}\left[\frac{\left|\Psi_{y}^{K+1}-\widetilde{\Psi}_{y}^{K+1}\right|}{\left|\Psi_{y}^{K+1}\right|}\right]<\delta_{t o l}
$$

na qual $\widetilde{\Psi}_{y}^{K+1}$ é a distribuição transversal calculada na iteração anterior. 


\section{EXEMPLOS BIDIMENSIONAIS E RESULTADOS}

\subsection{Guia planar não-linear e não-recíproco}

Os métodos FDTD e FD-BPM são aplicados na análise da propagação de modos TM (componentes $H_{y}, E_{x}$ e $E_{z}$ ) no mesmo guia não-linear e não-recíproco ilustrado na Fig. 4. Trata-se de um problema bidimensional, pois não há variação dos campos ao longo do eixo $y$ (propagação ao longo do eixo $z$ ). O efeito de não-reciprocidade não é observado para modos TE em virtude da direção adotada para o campo $\mathrm{H}_{\mathrm{DC}}$.

A configuração do guia planar é listada na TABELA 4. O comprimento de onda é $\lambda=1,152 \mu m$. O tamanho do domínio espacial numérico é $L_{x}=10,58 \mu m$ e $L_{z M A X}=1,0 \mathrm{~mm}$, acomodando um número total de células de $N=920 \times 10000$. No FDTD, $L_{z M A X}=100,4 \mu m$.

TABELA 4 - Parâmetros utilizados nas simulações

\begin{tabular}{|c|c|c|c|c|c|}
\hline Camada & $\begin{array}{c}\text { Índice de } \\
\text { refração }(n)\end{array}$ & Espessura & $\begin{array}{c}\text { Constante } \\
\text { Magneto- } \\
\text { óptica }(\delta)\end{array}$ & $\begin{array}{c}\text { Coeficiente } \\
\text { não-linear } \\
(\alpha)\end{array}$ & $\begin{array}{c}\text { Constante de } \\
\text { acoplamento } \\
\text { não-linear }(\gamma)\end{array}$ \\
\hline $\begin{array}{c}\text { cobertura não- } \\
\text { linear }\end{array}$ & 2,40 & $5,06 \mu m$ & - & $\begin{array}{c}6,377 \times 10^{-12} \\
\mathrm{~m}^{2} / \mathrm{V}^{2}\end{array}$ & $-1 / 2$ \\
\hline filme & 2,44 & $0,46 \mu m$ & - & - & - \\
\hline $\begin{array}{c}\text { substrato } \\
\text { magnetoóptico }\end{array}$ & 2,40 & $5,06 \mu m$ & $4,3 \times 10^{-3}$ & - & - \\
\hline
\end{tabular}

O que se espera com estes resultados é poder analisar as propriedades de nãolinearidade e não-reciprocidade do dispositivo. O índice efetivo $\left(\beta / k_{0}\right)$ do modo é computado a partir da variação longitudinal da componente $H_{y}$ na camada guiante, 
utilizando uma técnica de conversão para o domínio da freqüência, como a DFT. No FD-BPM, isto não foi possível devido à eliminação da variação rápida de campo (SVEA). Assim, o índice efetivo do modo é computado no FD-BPM a partir da integração da equação de onda (3.7) ao logo de todo o domínio transversal (eixo $x$ ) e fazendo $\partial H_{y} / \partial z=-j \beta H_{y}$, na qual $\beta$ é a incógnita. Dessa forma, é possível medir o índice efetivo utilizando a distribuição transversal de campo e de índice de refração para uma dada posição longitudinal.

$\mathrm{O}$ efeito da densidade de potência óptica $\left(P_{e x c}\right)$ sobre o índice efetivo, $\beta / k_{0}$, é mostrado na Fig. 8. Note que quando $P_{\text {exc }}$ aumenta, a espessura efetiva da camada guiante também aumenta devido ao efeito não-linear na camada de cobertura. Portanto, um deslocamento da distribuição de campo para o meio não-linear, e um conseqüente aumento do índice efetivo modal, irá ocorrer. Este deslocamento reduz a interação do campo óptico com o material magnetoóptico, provocando uma redução no desvio de fase $\phi$, conforme mostrado na Fig. 9. O desvio de fase não-recíproco é definido como $\phi=\left(2 \pi / \lambda_{0}\right)\left[\left(\beta / k_{0}\right)^{+}-\left(\beta / k_{0}\right)^{-}\right]$, na qual os subscritos "+" e "-" referemse aos modos TM propagantes no sentido direto e reverso, respectivamente.

Aumentando a densidade de potência óptica de entrada pode haver a indução de um canal guiante separado na região não-linear. A luz desviada para este canal pode interagir com a camada guiante original (se a condição de casamento de fase for obedecida), resultando em um acoplamento periódico entre o canal induzido e a camada guiante original. Este fenômeno interessante é ilustrado na Fig. 10(a) para $P_{\text {exc }}=125 \mathrm{~mW} / \mathrm{mm}$. Note que a onda propagante apresenta um acoplamento periódico, tal como em um acoplador direcional. Este comportamento afeta o índice efetivo medido ao longo da direção de propagação, como mostrado na Fig. 10(b). Se a densidade de potência é aumentada ainda mais, a constante de fase do modo propagante ao longo do canal induzido se torna evidentemente maior que a constante de fase do modo que propaga no filme linear. Portanto, a energia óptica segue pelo canal induzido sem acoplar de volta para o filme, conforme mostrado na Fig. 11 para $P_{\text {exc }}=303 \mathrm{~mW} / \mathrm{mm}$.

O tempo de processamento necessário para a obtenção da Fig. 11 em processador AMD Athlon ${ }^{\mathrm{TM}}$ XP 2600 de 2,08 GHz de clock, foi de 128 minutos e 47 segundos, utilizando o método FDTD, e de 4 minutos e 4 segundos, utilizando o 
método FD-BPM. Estes tempos confirmam a eficiência muito superior do FD-BPM na simulação de problemas unidirecionais de óptica guiada.

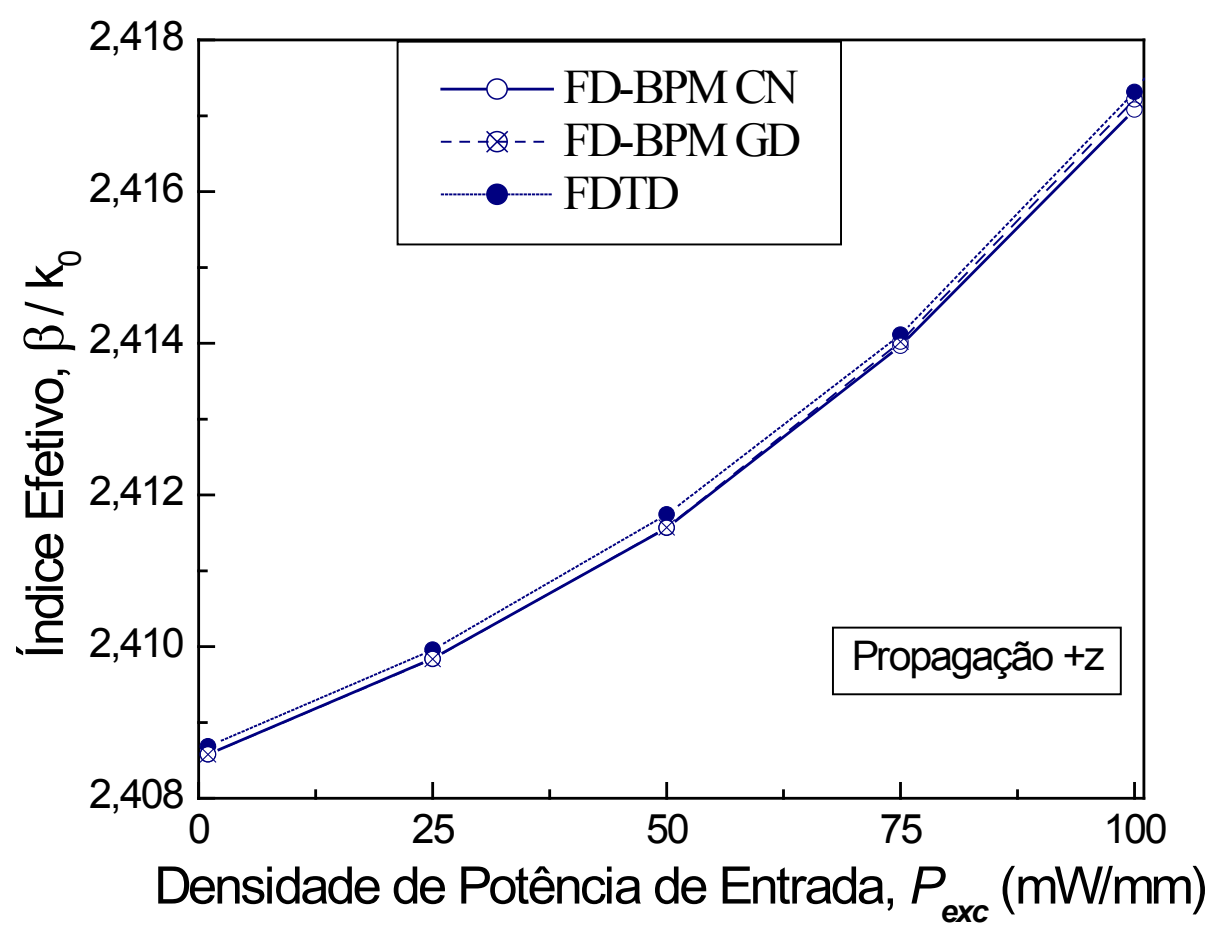

Fig. 8 - Índice efetivo modal em função da potência de entrada do laser.

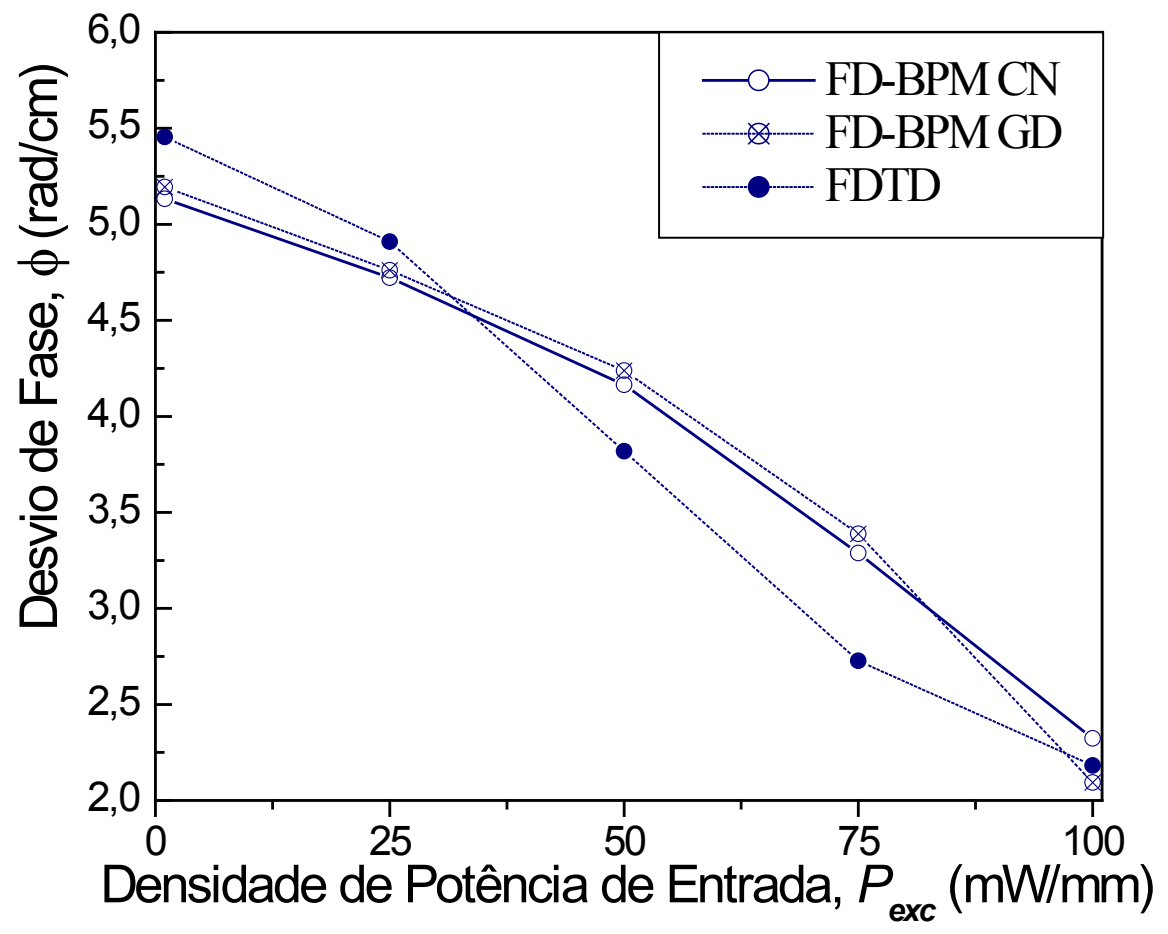

Fig. 9 - Desvio de fase não-recíproco em função da potência de entrada do laser. 


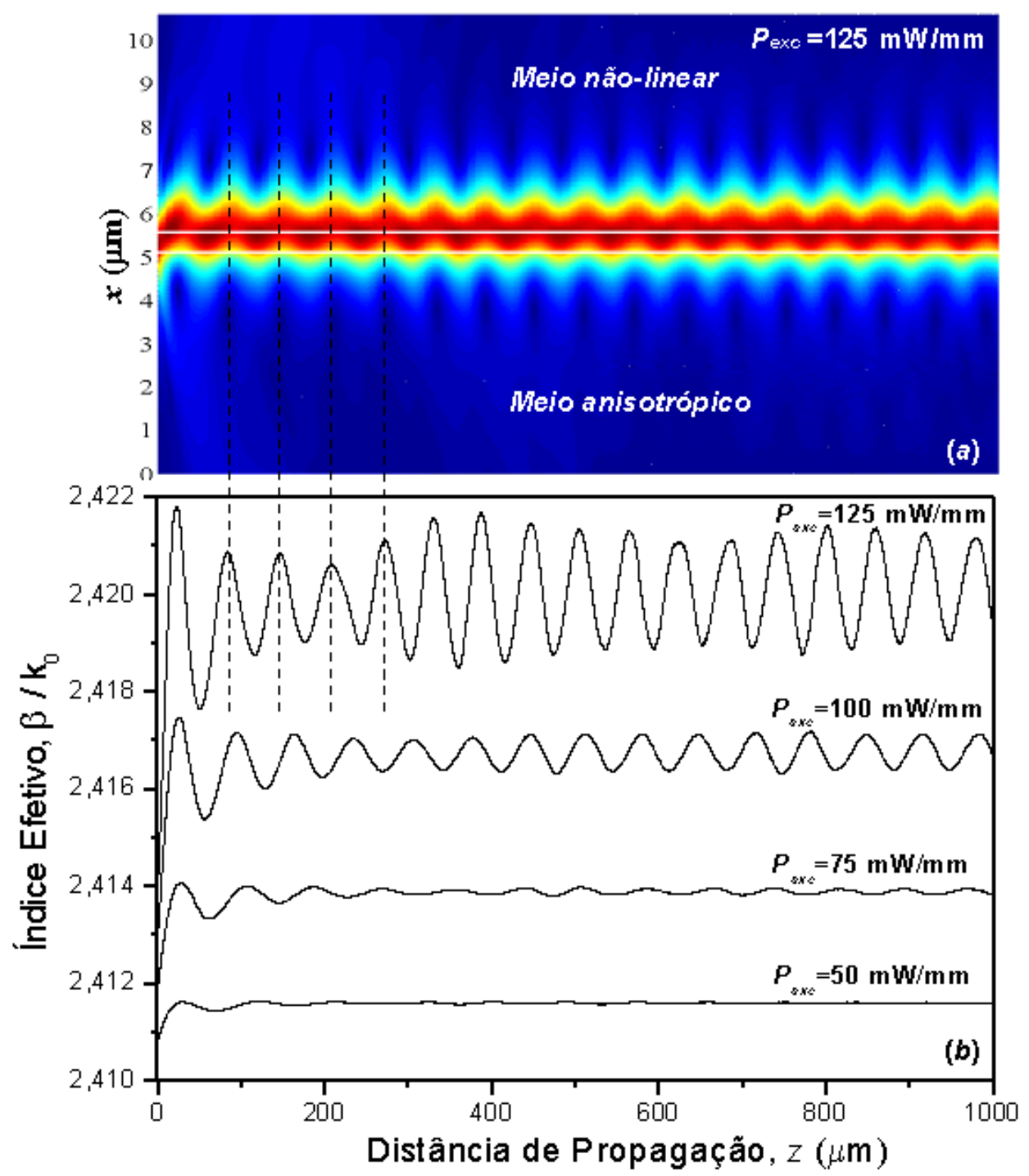

Fig. 10 - Comportamento modal em função do aumento da densidade de potência de entrada: (a) distribuição de $\left|H_{y}\right|$ para $\mathrm{P}_{\mathrm{exc}}=125 \mathrm{~mW} / \mathrm{mm}$ (linhas brancas delimitam o filme linear) e (b) índice efetivo em função da direção longitudinal z. 


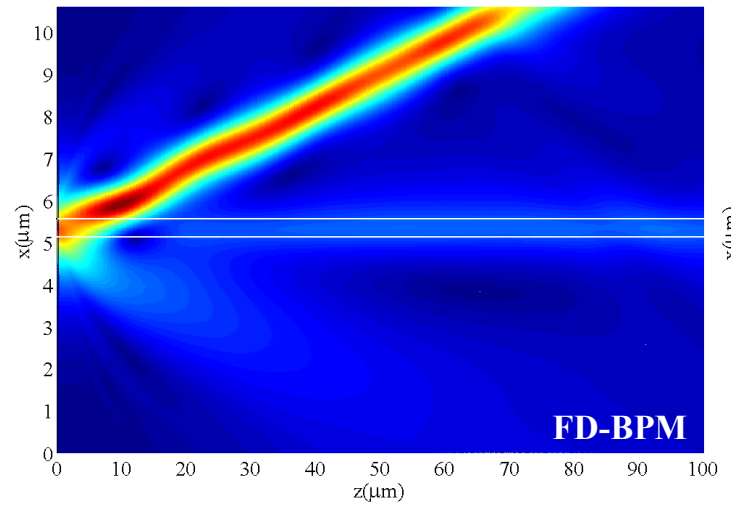

(a)

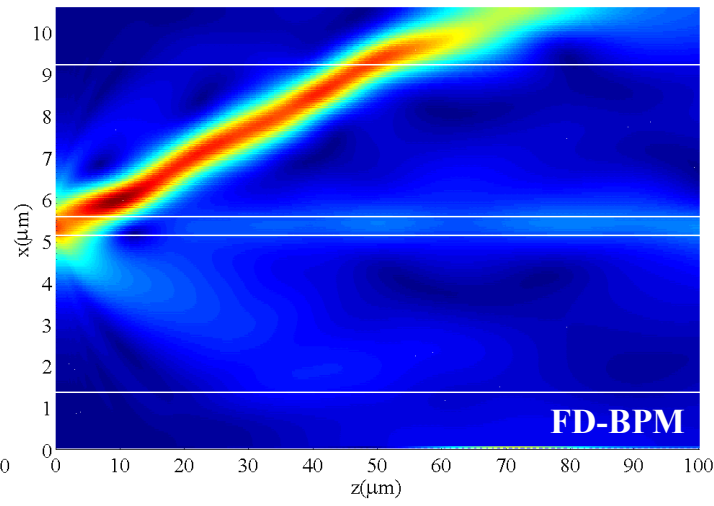

(b)

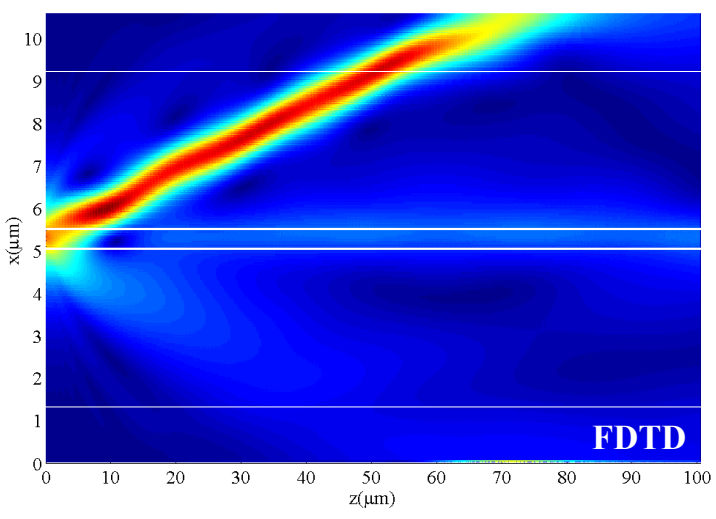

(c)

Fig. 11 - Canal induzido no meio não-linear para uma densidade de potência de entrada de $303 \mathrm{~mW} / \mathrm{mm}$. (a) Condição de contorno transparente,TBC, (b)-(c) Condição de contorno de camadas perfeitamente casadas, PML. As linhas centrais indicam a localização do filme guia de onda.

\subsection{Acoplador direcional não-linear e não-recíproco}

Nesta seção é proposto um novo dispositivo capaz de desempenhar as funções tanto de um chaveador quanto de um isolador óptico, simultaneamente. Este dispositivo foi projetado para modos com polarização TM e é, basicamente, um acoplador direcional caracterizado pelo fato de que a transferência de energia entre os guias é controlada pela densidade de potência óptica aplicada em sua entrada. A este aspecto foi adicionado o fenômeno da não-reciprocidade, proporcionando a característica híbrida ao dispositivo a qual será descrita em detalhes a seguir

O novo formalismo FD-BPM é empregado na análise deste dispositivo, o qual é baseado em um acoplador direcional constituído de três tipos de materiais simultaneamente, ou seja: linear, não-linear e magnetoóptico. O perfil da estrutura é 
ilustrado na TABELA 5 juntamente com o comportamento esperado para o fluxo de potência óptica (flechas). Os números de 1 a 4 identificam as portas de entrada/saída.

TABELA 5 - Requisitos do projeto.

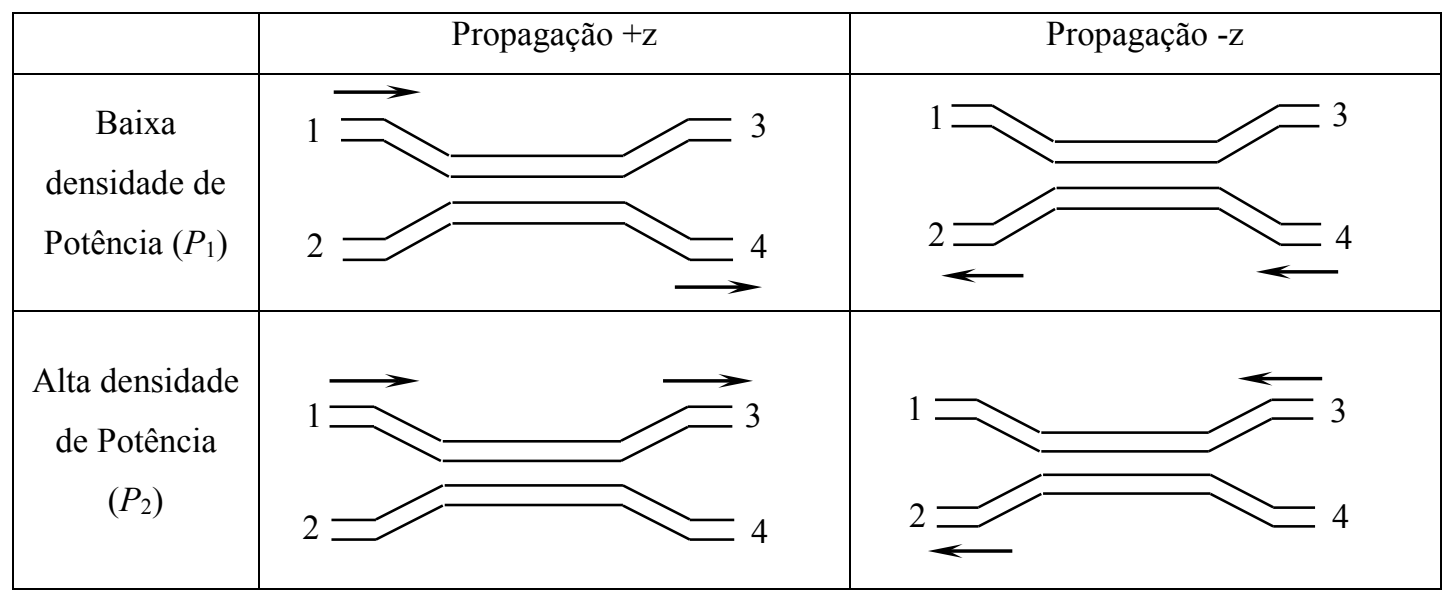

O que se pretende com este dispositivo é utilizar a propriedade não-linear do acoplador a ser projetado para proporcionar um chaveamento sempre que houver um aumento da densidade de potência de entrada (de $P_{1}$ para $P_{2}$ ). Ao mesmo tempo, a porta onde se encontra a excitação deve estar isolada de qualquer reflexão que por ventura ocorra nas portas de saída. Este comportamento evita que a luz refletida na saída seja novamente acoplada na porta de entrada. Caso contrário, poderá haver um desvio (indesejável) de freqüência (chirping) no laser. Por exemplo, no caso de potência baixa $\left(P_{1}\right)$ e propagação no sentido $+\mathrm{z}$, a potência é excitada na porta $1 \mathrm{e}$ deixa o dispositivo via porta 4. Em caso de reflexão (propagação -z), a potência refletida retorna pela porta 2 , proporcionando o isolamento da porta 1 .

Por se tratar de um acoplador direcional, o projeto da estrutura deve levar em conta a condição de casamento de fase entre os guias superior (guia A) e inferior (guia B) de modo a permitir máxima transferência de potência entre ambos para uma dada condição. Isto exige que os guias A e B sejam projetados considerando um isolado do outro (separados de uma distância infinita). Portanto, o projeto do acoplador é realizado em duas etapas.

Inicialmente foram atribuídas as características físicas e geométricas, índices de refração e espessuras, como mostrado na Fig. 12 e na TABELA 6. O índice de refração do núcleo do guia $\mathrm{B}\left(n_{4}\right)$ e o comprimento da região de interação $\left(\mathrm{L}_{z}\right)$ 
deverão ser definidos em função das características de guiamento do guia $\mathrm{A}$. O índice de refração da camada $3\left(n_{3}\right)$ foi escolhido ligeiramente inferior ao índice da camada $1\left(n_{1}\right)$ para aumentar o contraste de índice de refração em relação ao núcleo do guia A e, assim, uma maior parte da distribuição transversal de campo interagirá com o meio magnetoóptico. Isto permite uma maior diferença de fase na inversão do sentido de propagação. Com o guia A já especificado, e considerado isolado do guia $\mathrm{B}$, o próximo passo foi utilizar o próprio FD-BPM na obtenção do índice efetivo do modo guiado. Os detalhes da discretização utilizada bem como o tamanho da janela computacional são descritos na próxima seção.

porta 1 porta 3

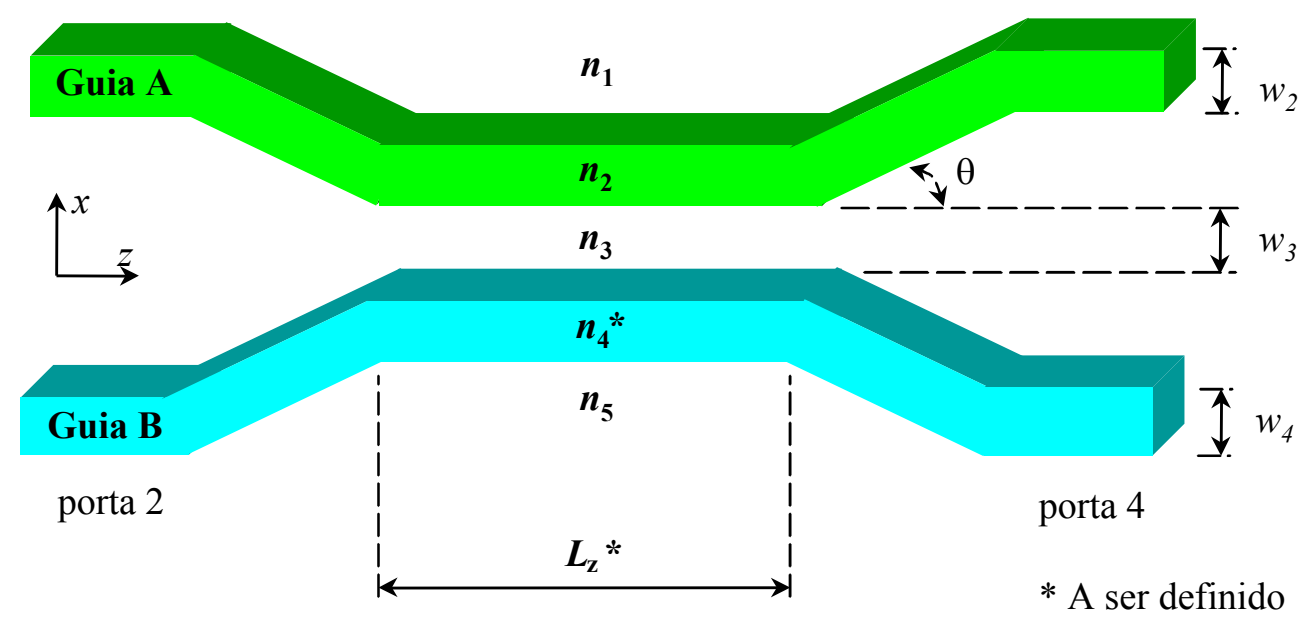

Fig. 12 - Esboço inicial do dispositivo chave/isolador. A faixa de interação entre os guias é delimitada por um ângulo de inclinação de $\theta=1,791^{\circ} \mathrm{e}$ o comprimento de onda de operação é $\lambda=1,32 \mu m$.

TABELA 6 - Parâmetros físicos do acoplador direcional

\begin{tabular}{|l||c|c|c|c|c|}
\hline Camada & $\begin{array}{c}\text { Índice de } \\
\text { refração } \\
(n)\end{array}$ & $\begin{array}{c}\text { Espessura } \\
(w)\end{array}$ & $\begin{array}{c}\text { Constante } \\
\text { Magneto- } \\
\text { óptica }(\delta)\end{array}$ & $\begin{array}{c}\text { Coeficiente não- } \\
\text { linear }(\alpha)\end{array}$ & $\begin{array}{c}\text { Constante de } \\
\text { acoplamento } \\
\text { não-linear }(\gamma)\end{array}$ \\
\hline 1 & 2,25 & $7,0 \mu m$ & $-0,18$ & - & - \\
\hline 2 (guia A) & 2,27 & $1,0 \mu m$ & - & $\varepsilon_{c}^{n l} c_{0} \varepsilon_{0} \cdot 10^{-9} \mathrm{~m}^{2} / \mathrm{V}^{2}$ & $-1 / 2$ \\
\hline 3 & 2,23 & $1,0 \mu m$ & - & - & - \\
\hline 4 (guia B) & $?^{*}$ & $1,0 \mu m$ & - & - & - \\
\hline 5 & 2,25 & $7,0 \mu m$ & - & - & - \\
\hline
\end{tabular}

* A ser definido 
Primeiramente, a densidade de potência óptica de excitação $P_{1}$ deve ser ajustada para $1 \mathrm{~mW} / \mathrm{mm}$ de modo que a região não-linear não produza nenhum efeito no guiamento modal. Assim, foram realizados cálculos de índice efetivo ao longo de uma distância longitudinal de $250 \mu \mathrm{m}$. O índice efetivo computado para o guia nãolinear e não-recíproco isolado (guia A) foi $\operatorname{neff}^{+}\left(P_{1}\right)=2,2552641$ para o sentido $+z \mathrm{e}$ $n_{\text {eff }}^{-}\left(P_{1}\right)=2,2514585$ para o sentido $-z$. A precisão na qual o índice efetivo é calculado através do FD-BPM é sensível ao passo transversal (esquema $\mathrm{CN}$ ) e às dimensões do domínio espacial numérico (no caso, as distâncias transversal e longitudinal devem ser suficientes para a convergência adequada do modo). A precisão também é dependente da eficiência da condição transparente ou absorvente utilizada nos limites numéricos. Um teste simples acerca da precisão do cálculo do índice efetivo modal através do método FD-BPM bidimensional é realizado no Apêndice E.

Uma vez conhecido o índice efetivo do guia A, o passo seguinte é projetar o guia linear e recíproco (guia B) tendo um índice efetivo o mais próximo possível de neff ${ }^{+}\left(P_{1}\right)$. Esta condição permite que toda energia do guia A seja transferida para o guia $\mathrm{B}$ (veja a condição para baixa densidade de potência $\left(P_{1}\right)$ ilustrada na TABELA 5). Fazendo $n_{4}=2,2737$, a relação de dispersão para o guia linear e recíproco de três camadas fornece $n e f f_{\mathrm{B}}=2,2552323 \approx n e f f^{+}\left(P_{1}\right)$.

Retornando ao guia A isolado, é sabido que o índice de refração dependente da intensidade de campo na região não-linear provoca o aumento do índice efetivo com a potência óptica. $\mathrm{O}$ que se pretende é estimar a potência $P_{2}$ que proporcione o casamento de fase com o guia linear no sentido $-z$, ou seja, neff ${ }^{-}\left(P_{2}\right) \approx$ neff $_{\mathrm{B}} \approx$ $n e f f^{+}\left(P_{1}\right)$. Conseqüentemente, isto deverá provocar um descasamento de fase modal entre os guias no sentido $+\mathrm{z}$, garantindo o funcionamento em $P_{2}$ mostrado na TABELA 5. A Fig. 13 ilustra a variação do índice efetivo modal do guia A isolado em função da potência de excitação para o sentido -z. 


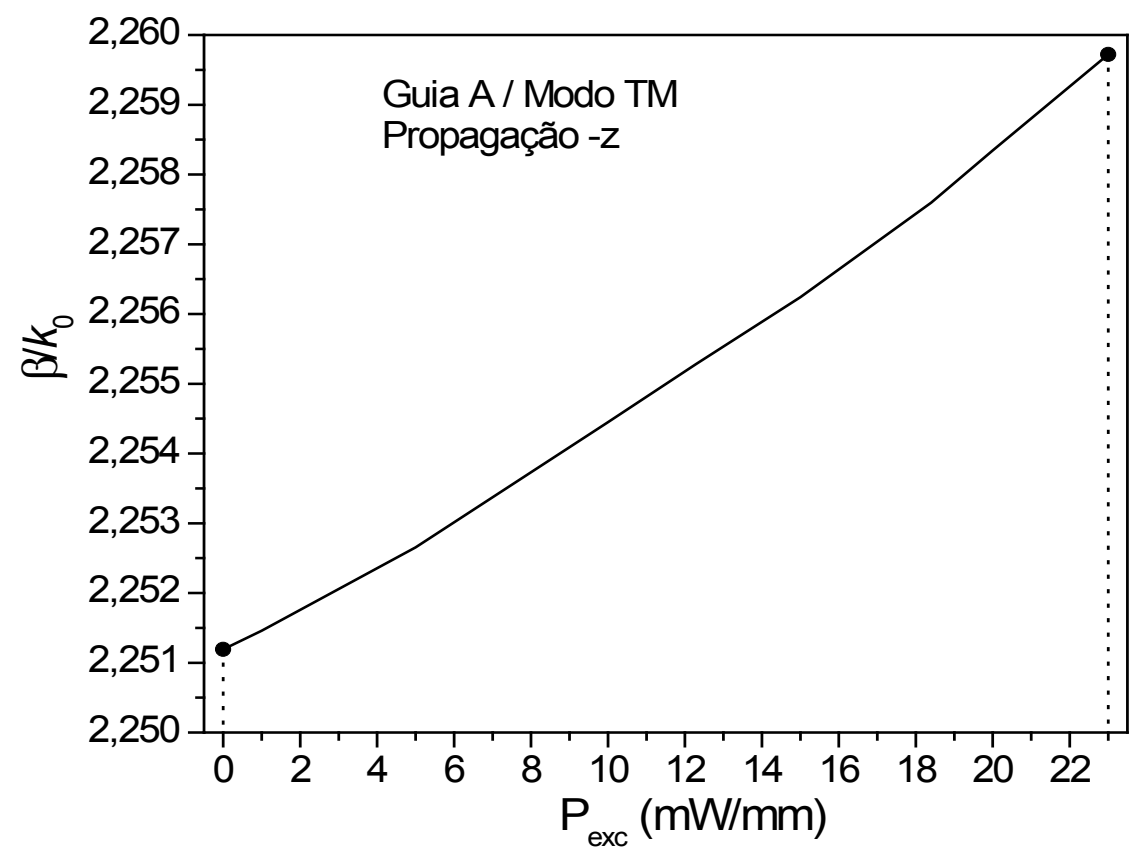

Fig. 13 - Índice efetivo em função da densidade de potência óptica de excitação para o guia não-linear e não-recíproco isolado (guia $\mathrm{A}$ ).

Quando há transferência de potência para outro guia, torna-se mais conveniente basear-se em um índice efetivo médio. Considerando transferência total de energia, a expressão para o índice efetivo médio utilizado é

$$
n e f f_{\text {avg }}^{-}\left(P_{2}\right)=\frac{1}{P_{2}} \int_{0}^{P_{2}} n e f f^{-}\left(P_{2}\right) d P .
$$

Por intermédio do gráfico da Fig. 13, e adotando $P_{2}=23,0 \mathrm{~mW} / \mathrm{mm}$, obtém-se $n e f f_{\text {avg }}^{-}\left(P_{2}\right) \approx 2,2551946$, que é similar ao índice efetivo do guia linear $\left(n e f f_{\mathrm{B}}\right)$.

Definidos os parâmetros dos materiais da estrutura ilustrada na Fig. 12, os resultados são verificados para a validação do projeto. Nas simulações envolvendo o FD-BPM foram atribuídos um passo transversal $\delta x=0,02 \mu m$ e um passo longitudinal $\delta z=0,2 \mu m$. Para limitar transversalmente o domínio espacial numérico foi aplicada a condição de contorno transparente (TBC). O comprimento de interação entre os guias, $L_{z}$ (Fig. 12), é ajustado convenientemente de acordo com as distâncias de acoplamento observadas. No caso deste projeto, $L_{z}=170 \mu m$.

A Fig. 14 mostra o fluxo da intensidade de campo para cada situação, indicando que o dispositivo atende as especificações de projeto. Observe que no 
caso de uma densidade de potência óptica $P_{1}$, a função de isolamento do acoplador deve-se mais à alteração da distância de acoplamento no sentido $-z$ do que ao descasamento de fase entre os guias. No caso de uma densidade de potência $P_{2}$, o efeito mencionado acontece na transmissão (sentido $+z$ ). Isto implica em uma dificuldade no ajuste da distância de iteração $L_{z}$. A redução ou aumento de $L_{z}$ pode melhorar o desempenho do dispositivo para um nível de potência, porém deteriorar sua performance quando operando no outro nível. Portanto, um ajuste fino deste parâmetro deve ser feito considerando todas as situações de operação de forma que um melhor compromisso seja alcançado. Este pode ser um trabalho árduo, onde poderia ser avaliada a possibilidade do uso de técnicas de otimização, como algoritmos genéticos. Contudo, intuitivamente, se uma maior diferença de fase nãorecíproca em $P_{1}$ for atingida, então esta dificuldade pode ser amenizada, pois nos casos $\left(-z, P_{1}\right)$ e $\left(+z, P_{2}\right)$ os descasamentos de fase seriam enfatizados.

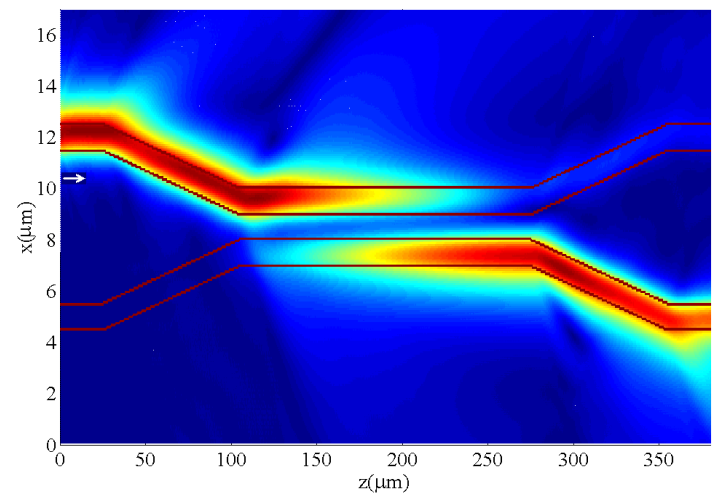

(a)

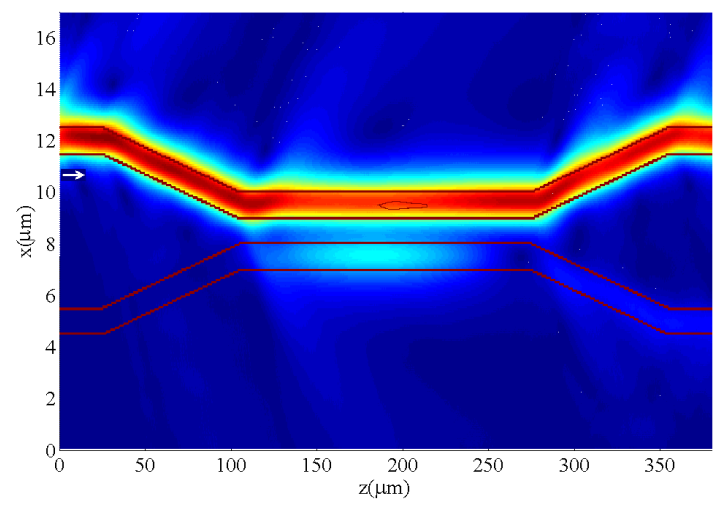

(c)

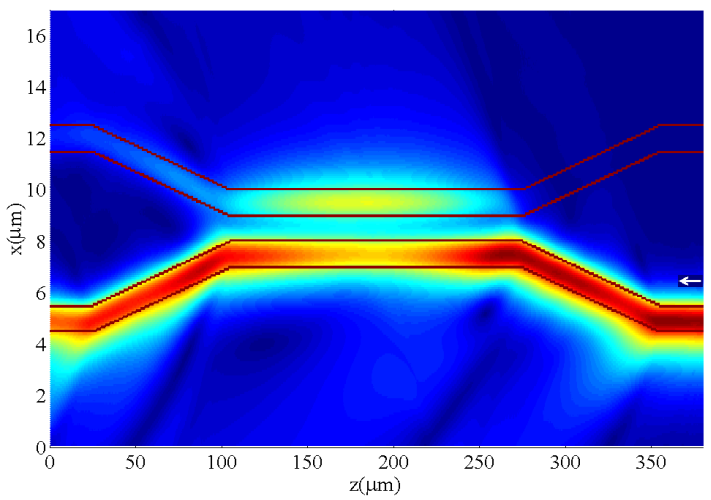

(b)

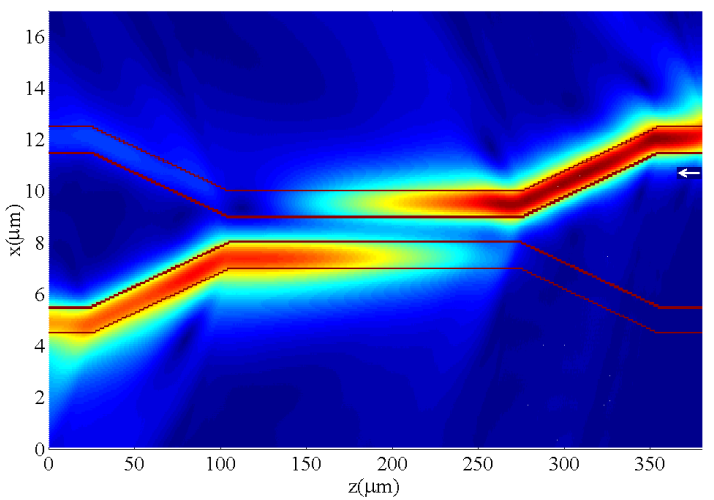

(d)

Fig. 14 - Fluxo de intensidade de campo para: (a) $\mathrm{P} 1=1,0 \mathrm{~mW} / \mathrm{mm}$ e propagação $+z$, (b) $\mathrm{P} 1=1,0 \mathrm{~mW} / \mathrm{mm}$ e propagação $-z$., (c) $\mathrm{P} 2=23,0 \mathrm{~mW} / \mathrm{mm}$ e propagação $+z$, (d) $\mathrm{P} 2=23,0 \mathrm{~mW} / \mathrm{mm} \mathrm{e}$ propagação $-z$. Compare com o diagrama da Tabela 5. 


\subsection{Efeito da não-reciprocidade sobre a emissão de sólitons}

Considere novamente um guia de três camadas planar, conforme mostrado na Fig. 15, na qual os parâmetros de não-linearidade são $\alpha=0,6377 \times 10^{-11}$ e $\gamma=1$. Foram realizados testes, utilizando o método FD-BPM, acerca da emissão de sólitons para alguns valores de constante magnetoóptica $\delta$, considerando uma potência de excitação $P_{\text {exc }}=280 \mathrm{~mW} / \mathrm{mm}$.

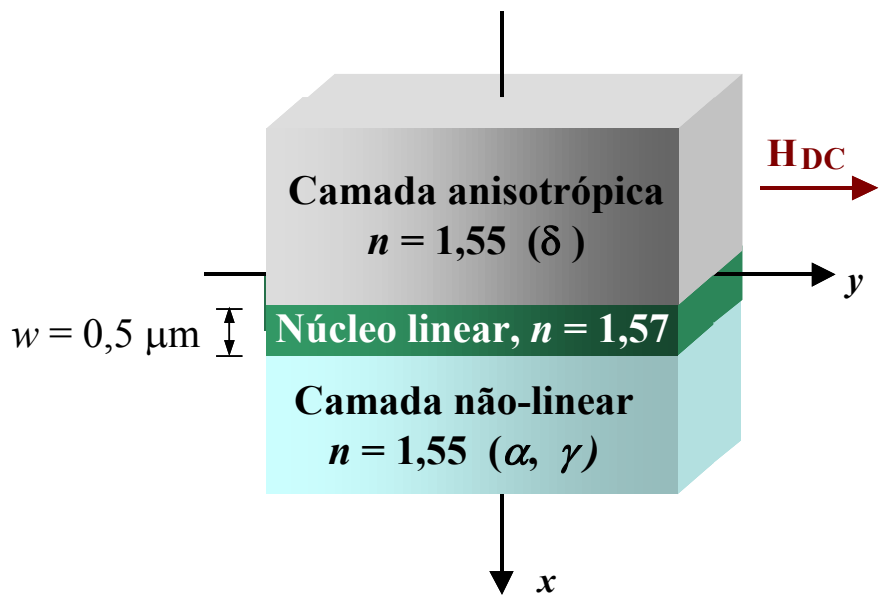

Fig. 15 - Guia planar não-linear e não-recíproco.

A Fig. 16 mostra o efeito do valor da constante magnetoóptica $\delta$ sobre a emissão de um sóliton. A Fig. 16(a) corresponde ao caso isotrópico ou à ausência de campo magnetostático $\mathrm{H}_{\mathrm{DC}}$, na qual a emissão do sóliton ocorre em $z \approx 120 \mu \mathrm{m}$. Este resultado é similar ao obtido por Yasui et al. [43], os quais analisaram um guia planar não-linear e isotrópico. Na Fig. 16(b) observa-se que a fixação do valor $\delta=$ 0,18 antecipa a emissão do sóliton para $\mathrm{z} \approx 80 \mu \mathrm{m}$. Na Fig. 16(c), a qual corresponde à inversão do campo $\mathrm{H}_{\mathrm{DC}}$ ou do sentido de propagação, não há emissão de sóliton na faixa longitudinal observada.

A fim de auxiliar a análise deste efeito, na Fig. 17 são exibidos gráficos do índice efetivo medido em função da distância de propagação, na qual a posição de emissão do sóliton é facilmente identificada pela transição abrupta do valor do índice efetivo medido. Observa-se nesta figura que o valor de $\delta$ também influi no valor inicial do índice efetivo medido. Para $\delta=0,18$ o índice efetivo inicial apresenta o menor valor e é o caso em que o sóliton é emitido na menor distância longitudinal. 
Contudo, para $\delta=-0,18$, o valor do índice efetivo inicial é o maior e a emissão de sóliton não é observada. Portanto, pode-se concluir que o valor da constante magnetoóptica afeta o valor do índice efetivo, aumentando ou diminuindo o confinamento modal, dependendo do sinal de $\delta$. Um menor confinamento antecipa a emissão do sóliton enquanto que o oposto retarda ou pode inibir sua emissão.

A Fig. 18 mostra o comportamento do índice efetivo medido para um valor absoluto de constante magnetoóptica dez vezes menor ( $|\delta|=0,018$ ), na qual observa-se que a variação do índice efetivo inicial para este valor de $|\delta|$ é menor, assim como a alteração na posição de emissão do sóliton. Para $\delta=0,018$, o sóliton é emitido em $z \approx 110 \mu \mathrm{m}$ e para $\delta=-0,018$, em $z \approx 130 \mu \mathrm{m}$.

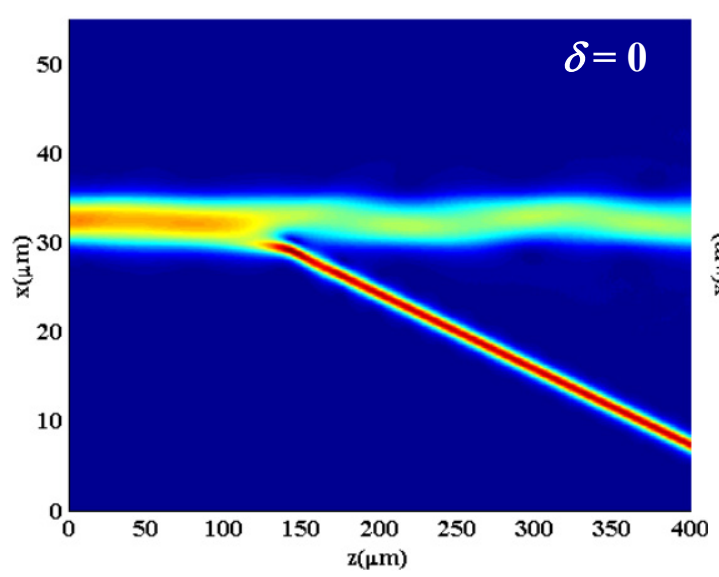

(a)

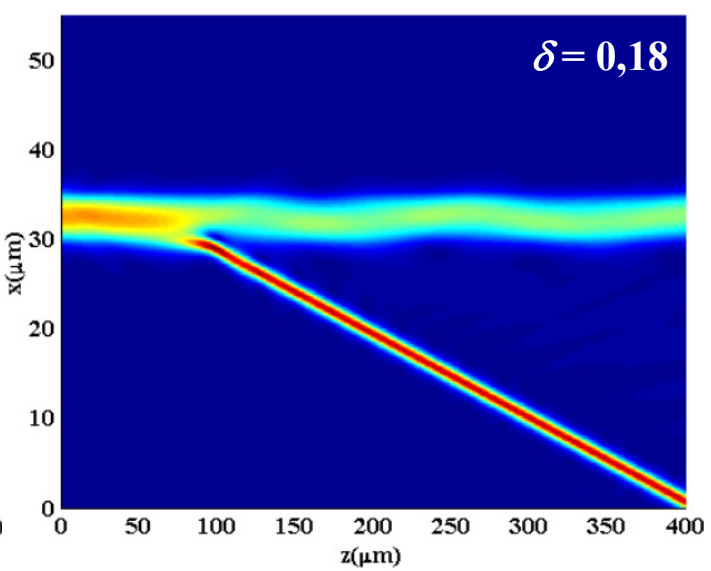

(b)

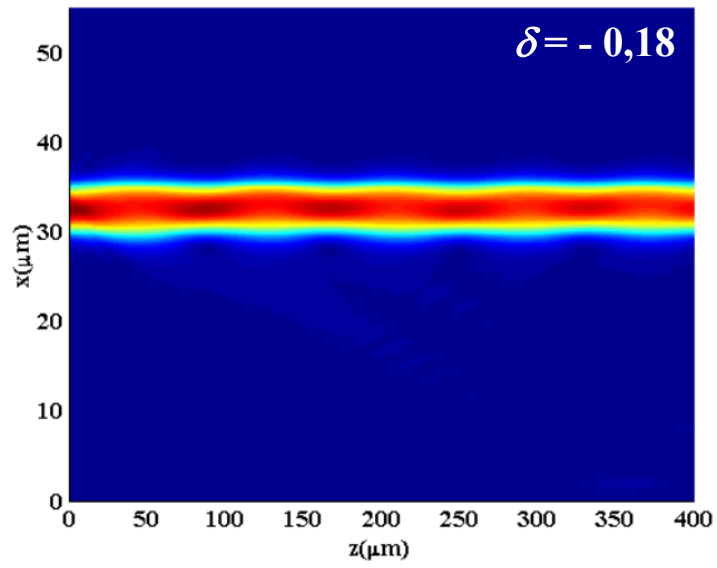

(c)

Fig. 16 - Efeito do valor da constante magnetoóptica $\delta$ sobre a emissão de sóliton para $P_{e x c}=280$ $\mathrm{mW} / \mathrm{mm}$. 


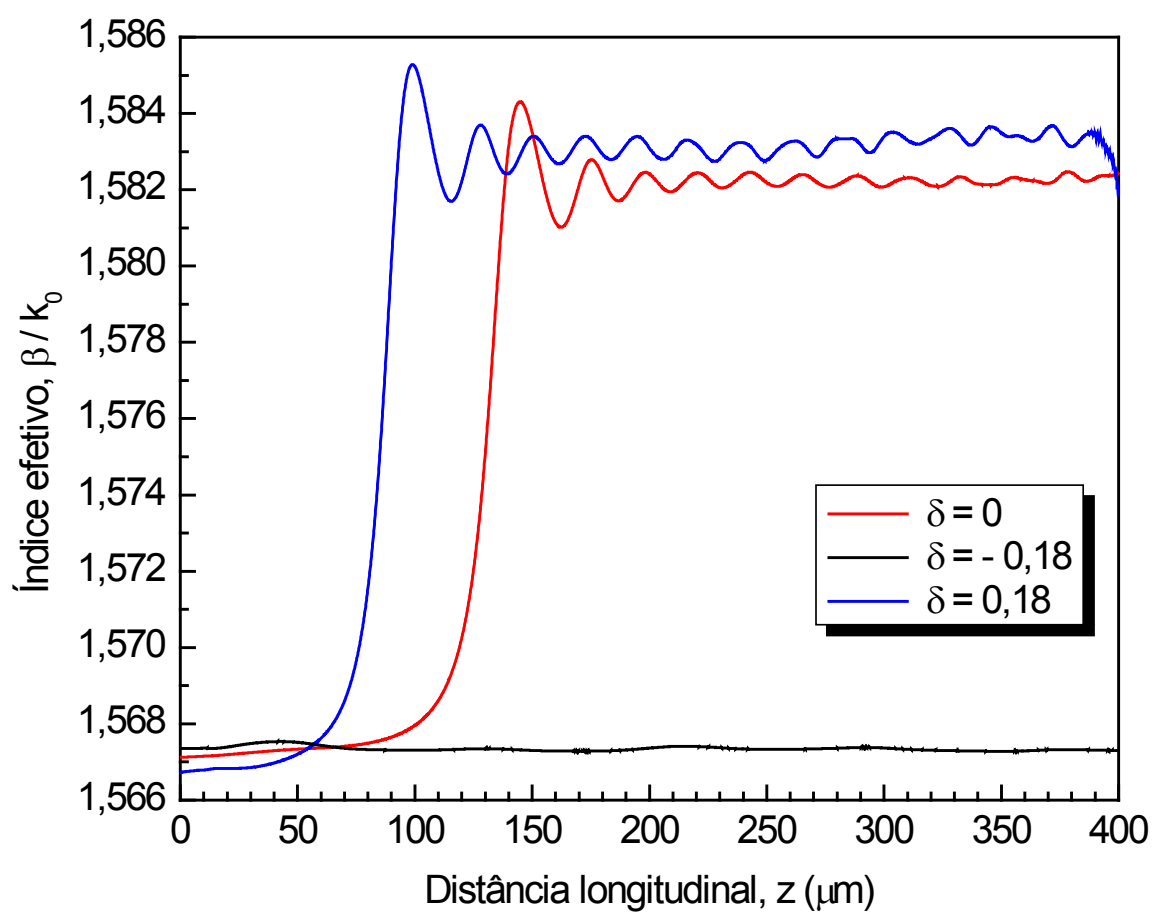

Fig. 17 - Variação do índice efetivo em função da distância de propagação correspondente às distribuições de campo mostradas na Fig. 16.

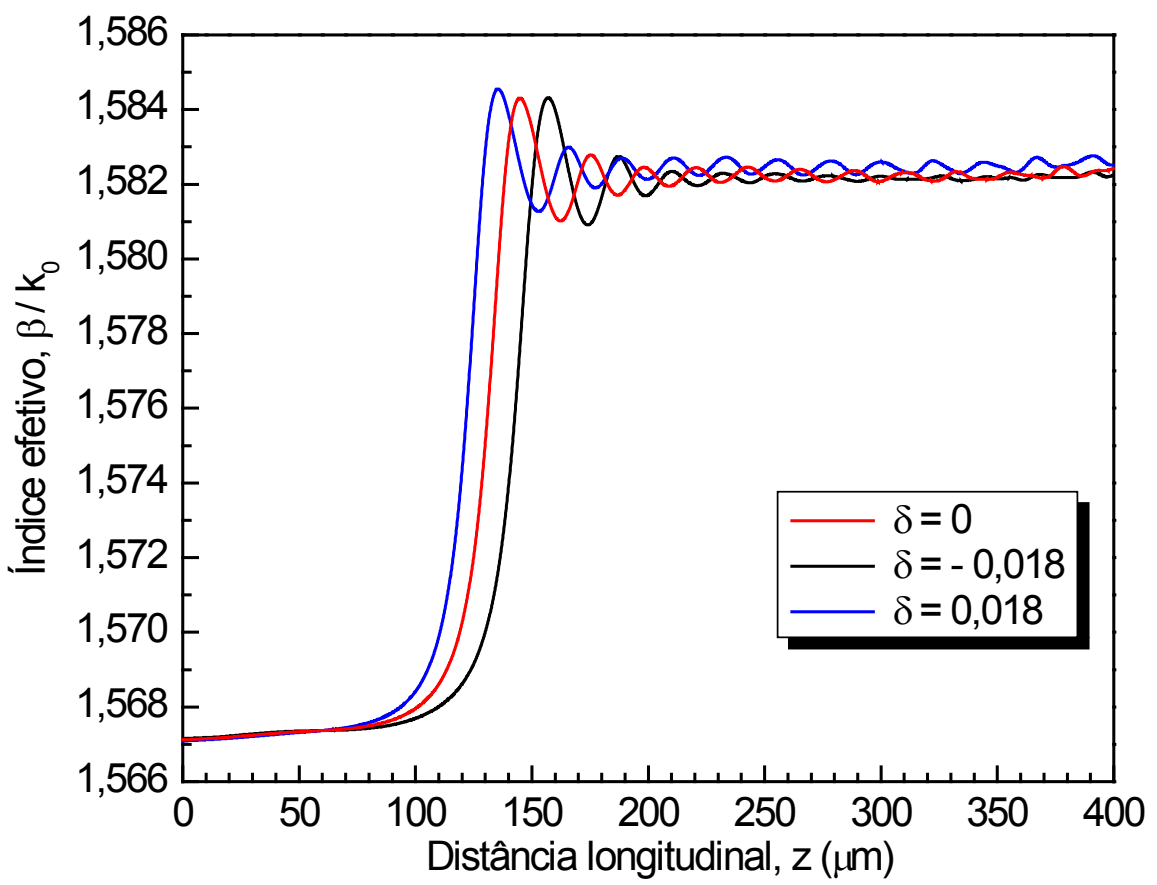

Fig. 18 - Variação do índice efetivo em função da distância de propagação para valor absoluto de $\delta$ dez vezes menor em relação à Fig. 17. 


\section{EXEMPLOS TRIDIMENSIONAIS E RESULTADOS}

Neste capítulo, são testadas as potencialidades método FD-BPM 3D vetorial. $\mathrm{Na}$ Seção 6.1 é analisado um isolador óptico baseado em um guia rib magnetoóptico. $\mathrm{Na}$ Seção 6.2, a extensão não-linear incorporada ao formalismo é verificada em uma extensa análise a respeito dos condensados de luz líquida que propagam em meios do tipo Kerr com saturação. A precisão da aplicação do método em uma estrutura isotrópica usual pode ser verificada no APÊNDICE F, onde uma fibra óptica convencional é vetorialmente analisada.

\subsection{Guia rib magnetoóptico}

O método proposto é validado na análise do guia rib magnetoóptico mostrado na Fig. 19. Esta estrutura foi originalmente projetada para atuar como um isolador óptico baseado em rotação de Faraday [44],[57].
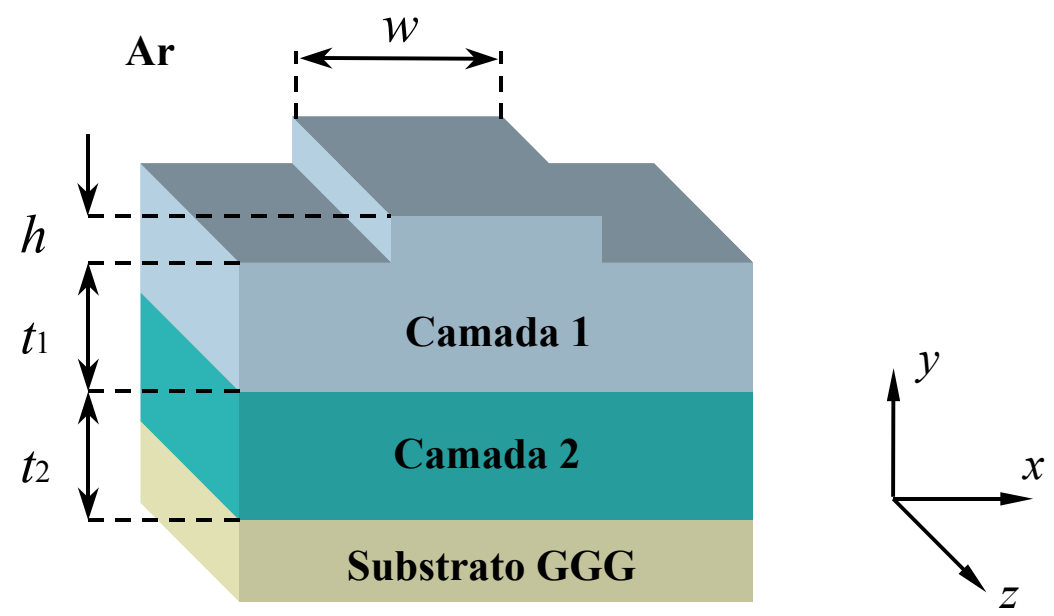

Fig. 19 - Guia rib magnetoóptico. 
Os parâmetros utilizados na simulação (exceto tensores permissividade) são detalhados na TABELA 7. As camadas 1 e 2 são compostas de Bi-YIG (bismuth yttrium iron garnet) crescidos epitaxialmente no topo de um substrato de GGG (gadolinium gallium garnet) $\mathrm{com} \varepsilon_{\mathrm{SR}}=(1,94)^{2}$. Para as camadas de Bi-YIG, o tensor permissividade é escrito como

$$
\overline{\bar{\varepsilon}}_{i}=\left(\begin{array}{ccc}
\varepsilon_{i x x} & j \delta & 0 \\
-j \delta & \varepsilon_{i y y} & 0 \\
0 & 0 & \varepsilon_{i z z}
\end{array}\right) \quad(i=1,2)
$$

TABELA 7 - Parâmetros para a análise numérica do guia rib magnetoóptico

\begin{tabular}{|c||c|c|}
\hline \multirow{2}{*}{ Parâmetro } & Descrição & Valor \\
\hline \hline$w$ & largura do núcleo do guia rib & $8,0 \mu \mathrm{m}$ \\
\hline$h$ & altura do núcleo do guia rib & $0,5 \mu \mathrm{m}$ \\
\hline$t_{1}$ & espessura da camada 1 (base do rib) & $3,1 \mu \mathrm{m}$ \\
\hline$t_{2}$ & espessura da camada 2 & $3,4 \mu \mathrm{m}$ \\
\hline$\lambda$ & comprimento de onda & $1,485 \mu \mathrm{m}$ \\
\hline$\delta x$ e $\delta y$ & passos transversais de discretização & $0,1 \mu \mathrm{m}$ \\
\hline$\delta z$ & passo longitudinal de discretização & $0,2 \mu \mathrm{m}$ \\
\hline$L x$ & largura do domínio espacial numérico & $80,0 \mu \mathrm{m}$ \\
\hline$L y$ & altura do domínio espacial numérico & $13,0 \mu \mathrm{m}$ \\
\hline$N x$ & número de células ao longo do eixo $x$ & 800 \\
\hline$N y$ & número de células ao longo do eixo $y$ & 130 \\
\hline
\end{tabular}

Os elementos da permissividade relativa tensorial representada em (6.1), para as camadas 1 e 2 , são dados por $\varepsilon_{1 x x}=\varepsilon_{1 z z}=(2,19-\Delta \mathrm{g})^{2}, \varepsilon_{1 y y}=(2,19)^{2}, \varepsilon_{2 x x}=\varepsilon_{2 z z}=$ $(2,18-\Delta \mathrm{g})^{2}, \varepsilon_{2 y y}=(2,18)^{2}[44]$.

O parâmetro $\Delta \mathrm{g}$ refere-se ao ajuste da birrefringência geométrica aplicado com o objetivo de compensar a birrefringência linear, esta última ocorrendo devido à deformação causada por compressão. Isto é necessário para que a máxima isolação 
no comprimento de onda desejado seja obtida. Aqui foi atribuído $\Delta \mathrm{g}=2,2 \times 10^{-4}$ [44]. Os dois termos fora da diagonal principal ocorrem devido a um campo magnetostático $H_{D C}$ aplicado ao longo da direção de propagação $z$. A constante magnetoóptica $\delta$ é associada com o ângulo de rotação de Faraday $\left(\Theta_{F}\right)$ e o índice de refração do meio $(n)$ por

$$
\delta=\frac{n \lambda \Theta_{F}}{\pi}
$$

na qual $\Theta_{F}=133 \%$ cm [57].

O efeito de rotação de Faraday é responsável por uma transferência de potência periódica entre as componentes transversais $E_{x}$ e $E_{y}$. A estrutura foi excitada em z=0 com a componente $E_{x}$ (supondo $E_{y}=0$ ), utilizando o método de sobre-relaxação sucessiva (SOR). A componente longitudinal $E_{z}$ é diretamente obtida por meio de $E_{x}$, $E_{y}$ e usando a lei de Gauss, Eq. (4.6). As distribuições transversais para as três componentes de campo elétrico foram monitoradas ao longo da distância necessária para a transferência total de potência, o que equivale a $90^{\circ}$ de rotação, de modo que algumas etapas são mostradas na Fig. 20. Conforme era esperado, a componente $E_{y}$ é estimulada devido aos elementos do tensor permissividade fora da diagonal principal.

O mecanismo de transferência de energia também pode ser observado pela análise da intensidade das componentes transversais $I_{x}$ e $I_{y}$, as quais podem ser obtidas como segue

$$
I_{u}(z)=\iint\left|E_{u}(x, y, z)\right|^{2} \partial x \partial y \quad(u=x, y) .
$$

O comprimento para a máxima transferência de energia é de aproximadamente $6800 \mu \mathrm{m}$ conforme mostrado na Fig. 21. Na prática, conforme é observado em [57], o comprimento do dispositivo deve ser ajustado para a metade deste valor $(\sim 3400 \mu \mathrm{m})$ de forma que uma rotação de $45^{\circ}$ seja atingida na porta de saída. Um polarizador posicionado na saída também a $45^{\circ}$ garantirá que toda a potência seja transmitida. Adicionalmente, qualquer campo refletido que entre na porta de saída do dispositivo também estará polarizado de $45^{\circ}$, o qual completará uma rotação de $90^{\circ}$ na porta de entrada e, conseqüentemente, estará apto a ser bloqueado por outro polarizador sem afetar o campo de entrada. O período de transferência de energia entre as componentes transversais encontrado para esta estrutura também é concordante ao 
observado por Selleri et al. [44], os quais analisam o desempenho do dispositivo por meio das componentes de campo magnético, utilizando um BPM vetorial baseado em elementos finitos.
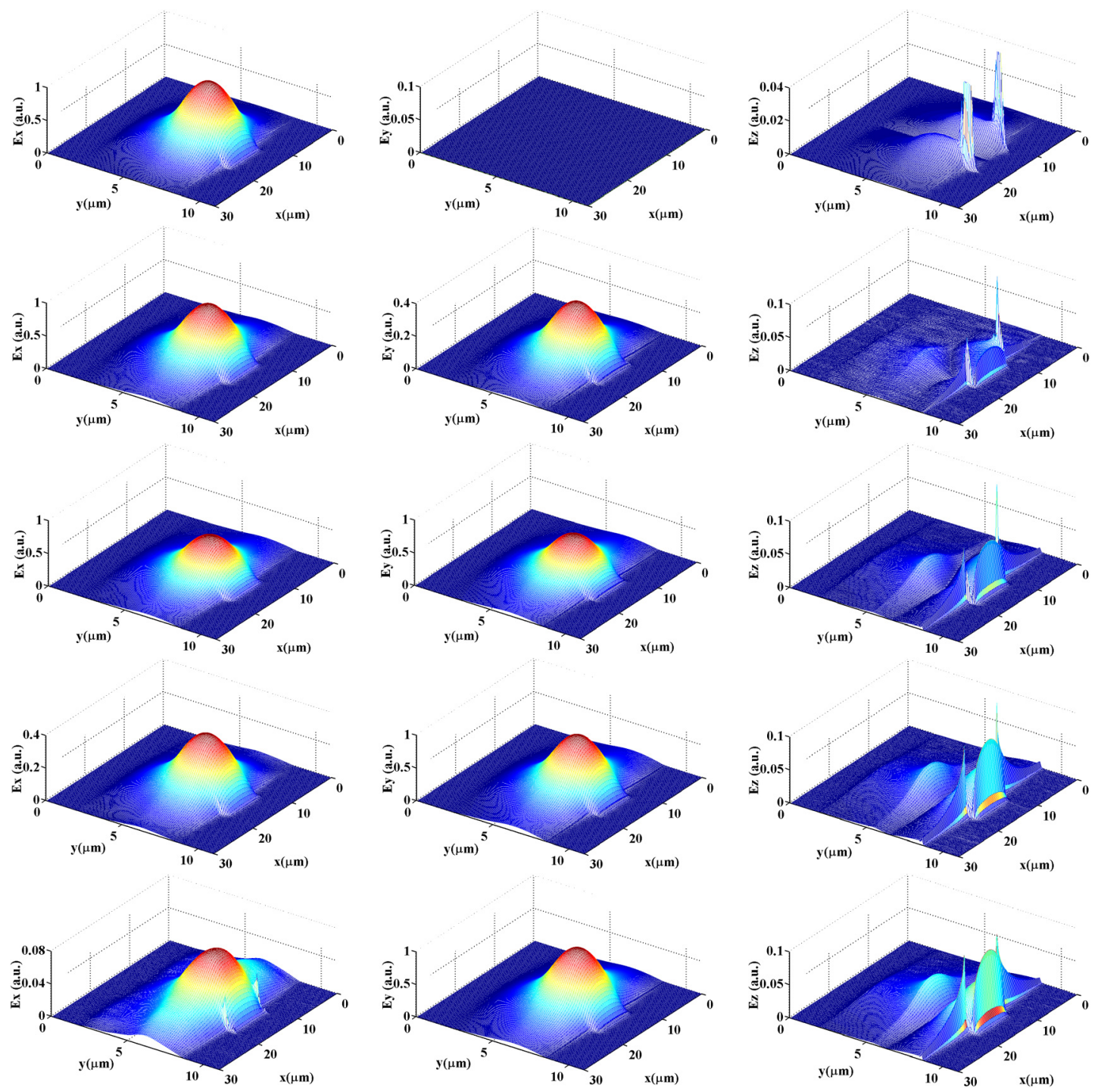

Fig. 20 - Distribuições transversais de campo para as componentes $E_{x}$ (coluna à esquerda), $E_{y}$ (coluna central) e $E_{z}$ (coluna à direita) em z $=0 \mu \mathrm{m}, 1700 \mu \mathrm{m}, 3400 \mu \mathrm{m}, 5100 \mu \mathrm{m}$, e $6800 \mu \mathrm{m}$.

O tempo de processamento necessário para simular a propagação eletromagnética em uma distância longitudinal de $7000 \mu \mathrm{m}$ foi de aproximadamente 235 minutos. Foi utilizado um processador AMD Athlon ${ }^{\mathrm{TM}}$ XP 2600 de 2,08 GHz de clock. Isto mostra a viabilidade da aplicação do método FD-BPM na análise numérica de guias tridimensionais longos (em comparação ao comprimento de onda) utilizando computadores convencionais. 


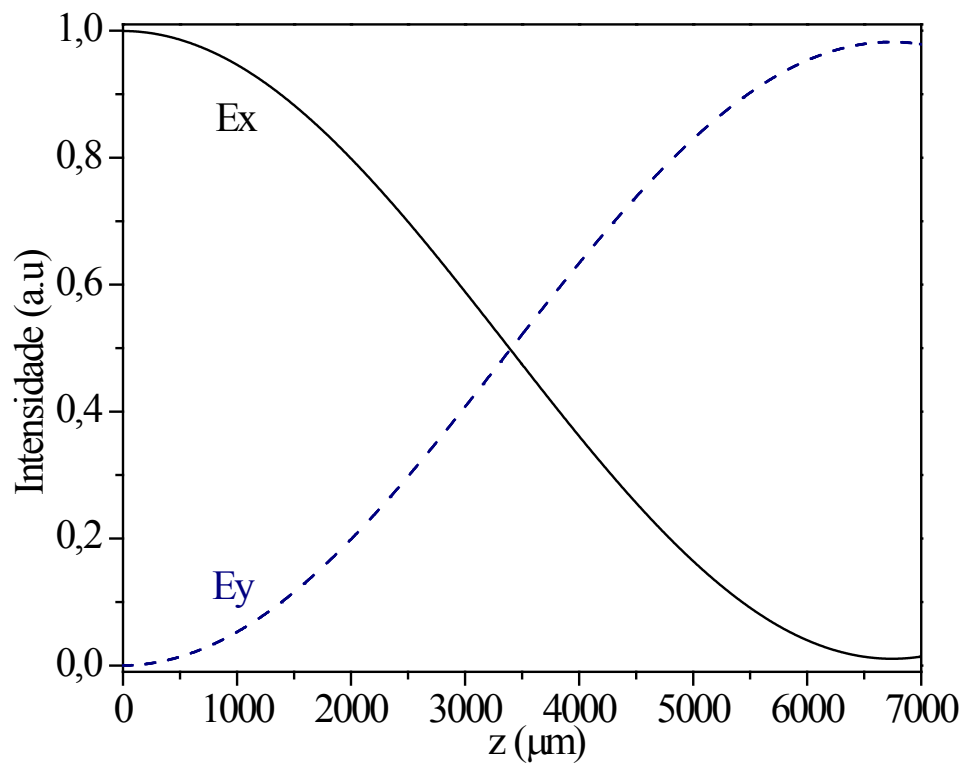

Fig. 21 - Evolução da intensidade normalizada das componentes transversais de campo elétrico ao longo do eixo de propagação do guia magnetoóptico.

\subsection{Condensados de luz "líquida"}

Nesta seção, a extensão para consideração de não-linearidades incorporada ao método FD-BPM 3D vetorial proposto é validada com a investigação da propagação óptica em material do tipo Kerr com saturação. A adição do termo de saturação garante a estabilidade na propagação de sólitons bidimensionais e tridimensionais formados nestes meios não-lineares.

Devido ao fato de apresentarem um limiar de energia crítico (abaixo do qual difratam e acima, se tornam auto-confinados), sólitons são candidatos naturais para a transmissão de informação binária. Ao contrário de feixes propagando em meios lineares, sólitons tem a capacidade de interagirem entre si, o que pode formar a base da implementação de computação digital totalmente óptica [58]. Com isso, torna-se imprescindível o aprimoramento das técnicas de simulação numérica com o objetivo de investigar novos efeitos não-lineares. $\mathrm{O}$ mais recente candidato a este posto são os condensados de luz líquida analisados por Michinel et al. [59], os quais estavam interessados em responder as seguinte perguntas: 1) É possível produzir algo que se 
assemelhe a um estado de luz líquida?, 2) Que propriedades físicas teriam este estado? Para tentar responder estas perguntas os autores consideraram um meio nãolinear do tipo Kerr com saturação, também conhecido como cúbico-qüíntico (CQ). Nestes meios o índice de refração aumenta com o aumento da densidade de potência óptica até que o ponto de saturação seja atingido. A partir deste ponto, ainda aumentando a densidade de potência óptica, o valor do índice de refração inverte sua tendência e começa a cair.

Em meios não-lineares do tipo Kerr com variação linear positiva do índice de refração (focalizante), a propagação de feixes bidimensionais intensos irá produzir efeitos indesejáveis tais como auto-focalização catastrófica e explosão [59]. Este colapso do feixe não ocorre em materiais não-lineares CQ. Com a investigação da propagação de feixes intensos nestes materiais foi possível comprovar que o perfil óptico produzido nestes materiais apresentava tensões superficiais semelhantes às observadas em líquidos. Assim, a propagação de um feixe de luz se comportaria como um fluxo de líquido, enquanto que a propagação de um pulso de luz se comportaria como uma gota de líquido, daí o fato de poderem ser interpretadas como condensados de luz. As tensões superficiais observadas nestes condensados correspondem, portanto, às mesmas forças de Van der Waals que mantém a integridade de uma gota de líquido. Materiais que serão fortes candidatos a este fim são os vidros calcogenetos (chalcogenide glasses), tal como o investigado por Frederic Smektala et al. [60], o qual apresentaria um índice de refração dependente de intensidade que poderia ser representado por (4.37). A investigação conduzida em [59] teve como base a equação não-linear de Schrödinger em sua forma escalar expandida no limite paraxial.

A TABELA 8 lista os valores dos parâmetros utilizados nas simulações com o método FD-BPM 3D vetorial, na qual os parâmetros do meio não-linear referem-se à equação (4.37). A variação do índice de refração com a magnitude de campo elétrico para estes parâmetros de não-linearidade é mostrada na Fig. 22. Quando sua amplitude de campo elétrico supera o ponto de saturação o feixe assume um formato supergaussiano, passando a apresentar um perfil estacionário de topo largo e plano [59]. 
TABELA 8 - Parâmetros usados na simulação de condensados ópticos

\begin{tabular}{|c||c||c|}
\hline \multicolumn{1}{|c||}{ Parâmetro } & Descrição & Valores \\
\hline \hline$\varepsilon_{L}$ & permissividade estática do meio não-linear & 3,24 \\
\hline$\alpha_{2}$ & coeficiente não-linear focalizador & $1,72 \times 10^{-18} \mathrm{~m}^{2} / \mathrm{V}^{2}$ \\
\hline$\alpha_{4}$ & coeficiente não-linear desfocalizador & $4,086 \times 10^{-35} \mathrm{~m}^{4} / \mathrm{V}^{4}$ \\
\hline$\lambda$ & comprimento de onda & $1,6 \mu \mathrm{m}$ \\
\hline$\delta x$ & passo transversal ao longo do eixo $x$ & $0,5 \mu \mathrm{m}$ \\
\hline$\delta y$ & passo transversal ao longo do eixo $y$ & $0,5 \mu \mathrm{m}$ \\
\hline$\delta z$ & passo de propagação & $0,2 \mu \mathrm{m}$ \\
\hline$L x$ & largura do domínio espacial numérico & $150 \mu \mathrm{m}$ \\
\hline$L y$ & altura do domínio espacial numérico & $150 \mu \mathrm{m}$ \\
\hline$N x$ & número de células ao longo do eixo $x$ & 300 \\
\hline$N y$ & número de células ao longo do eixo $y$ & 300 \\
\hline
\end{tabular}

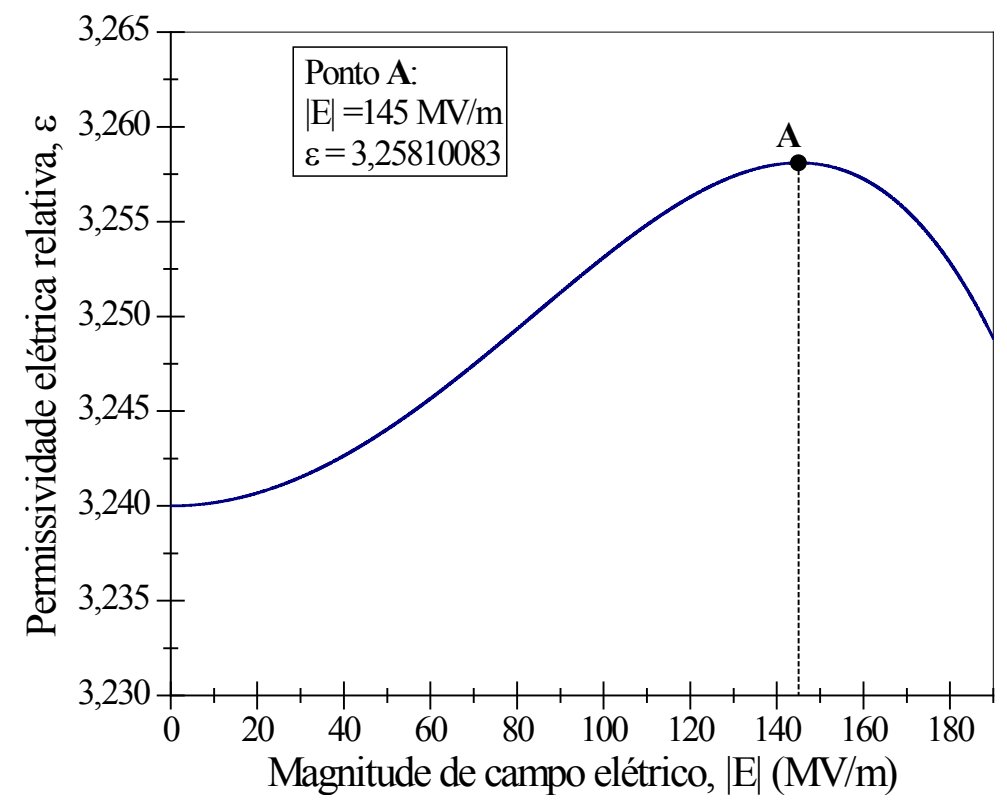

Fig. 22 - Variação da permissividade elétrica relativa com o módulo do campo elétrico no meio nãolinear. O ponto $\mathbf{A}$ indica o estado de saturação.

As simulações aqui realizadas têm como intuito verificar a teoria elaborada em [59] e analisar novos fenômenos, testando, ao mesmo tempo, as potencialidades do 
método FD-BPM 3D vetorial proposto. Na Seção 6.2.1 é verificado vetorialmente como um feixe gaussiano comum de alta amplitude se comporta na medida em que se propaga em um meio não-linear com saturação. Isto permitirá que seja adquirido um maior conhecimento dos atributos do sóliton formado neste meio, os quais serão utilizados para aplicar uma excitação inicial mais refinada nas próximas análises.

A fim de observar a analogia entre a propagação óptica de alta potência neste meio e a dinâmica dos fluidos, a Seção 6.2.2 apresenta os resultados para a incidência de um feixe de luz condensada em uma interface com o ar. A Seção 6.2.3, por sua vez, destaca a colisão entre dois feixes ópticos condensados para os casos em que os sólitons são excitados em fase e com uma defasagem de $180^{\circ}$. Isto se deve ao interesse científico em pesquisar e explorar as propriedades colisionais de gotas ópticas para o projeto de portas lógicas ópticas. Finalmente, a Seção 6.2.4 investiga a passagem de um feixe óptico condensado através de uma fenda metálica a fim de verificar a influência da difração imposta sobre o estado condensado da luz. Estes resultados servem como um teste de robustez na conservação da informação.

\subsubsection{Estabilização de um feixe gaussiano}

Nesta simulação, a componente $E_{x}$ é excitada com um feixe gaussiano com amplitude de $145 \mathrm{MV} / \mathrm{m}$ e largura de $15 \mu \mathrm{m}$ (valor RMS). Este feixe inicial converge para um formato supergaussiano após aproximadamente $\mathrm{z}=2500 \mu \mathrm{m}$, como pode ser observado na Fig. 23. A partir deste ponto sua amplitude oscila entre $171 \mathrm{MV} / \mathrm{m}$ e 192MV/m. O diâmetro do topo da supergaussiana resultante é de aproximadamente $22 \mu \mathrm{m}$. A distribuição final (à direita) foi tomada em $\mathrm{z}=8100 \mu \mathrm{m}$. A variação do índice de refração induzido pela propagação da supergaussiana é mostrada na Fig. 24, na qual a operação na região de saturação é confirmada pelo formato côncavo do guia induzido. A representação vetorial da supergaussiana resultante é mostrada na Fig. 25. 

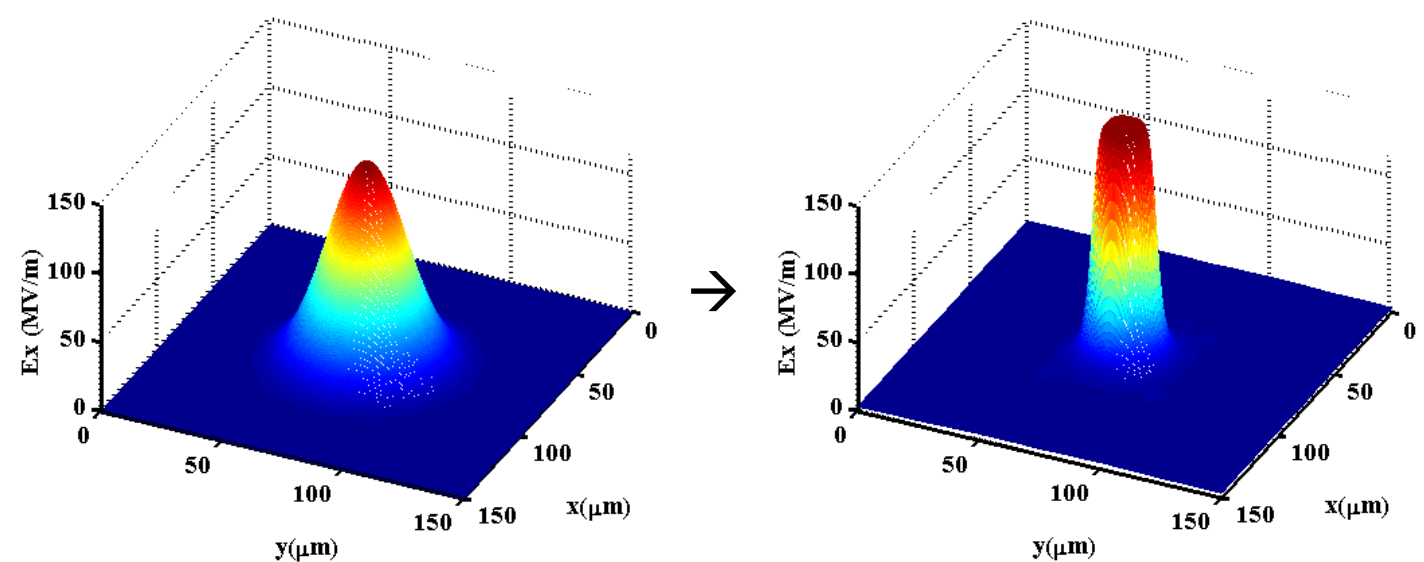

Fig. 23 - Transição de um pulso gaussiano de alta intensidade (visto em $z=0$ ) a um formato supergaussiano (visto em $z=8100 \mu \mathrm{m}$ ) em um meio do tipo Kerr com saturação.
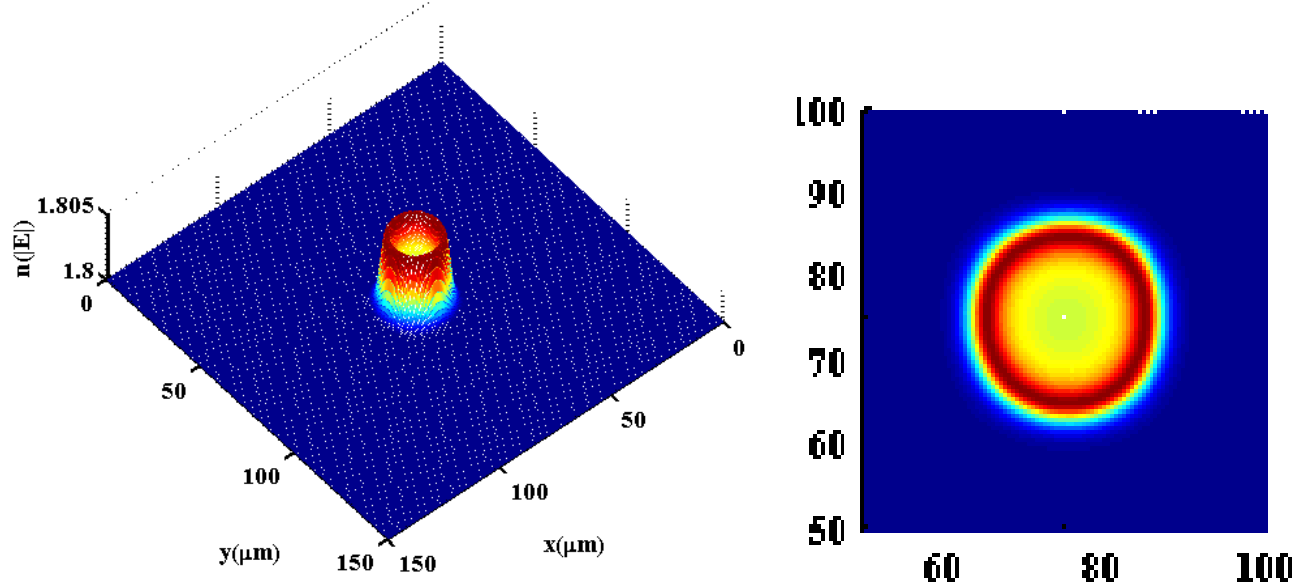

Fig. 24 - Distribuição de índice de refração dependente da intensidade de campo elétrico, devido à propagação da supergaussiana mostrada na Fig. 3 ( $\mathrm{z}=8100 \mu \mathrm{m})$. O núcleo côncavo surge como uma peculiaridade do meio não-linear cúbico-quíntico.

A emissão de campos radiados foi observada durante o estágio intermediário deste processo. Isto é análogo ao que ocorre, em termos de ondas guiadas, se o formato do campo excitado não corresponde exatamente ao formato do modo propagante para um dado guia de ondas. Como as amplitudes destes campos são naturalmente muito inferiores à da excitação, a comparação do comportamento destes campos radiados com o que ocorre na "dinâmica dos fluidos" não é válida (não há a formação de condensados de luz neste caso). 

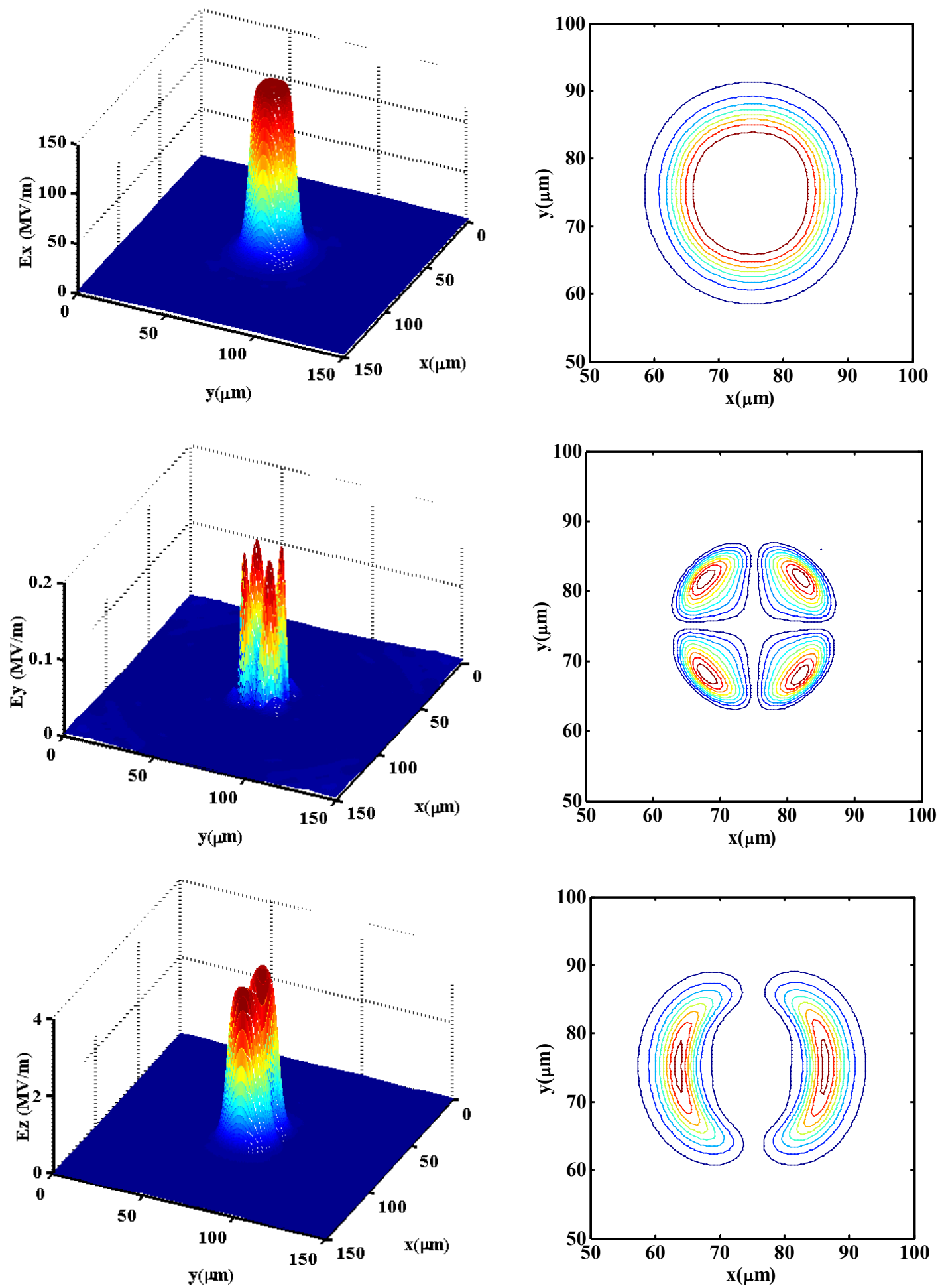

Fig. 25 - Componentes $E_{x}, E_{y}$ e $E_{z}$ da supergaussiana mostrada à direita da Fig. 23 (z=8100 $\left.\mu \mathrm{m}\right)$. 


\subsubsection{Colisão de um feixe supergaussiano em uma interface com o ar}

A fim de minimizar a emissão de campos radiados nas simulações que se seguem, o feixe inicial será caracterizado por um formato supergaussiano, o qual pode ser representado pela seguinte função:

$$
E_{x}=A_{0} \exp \left[-\frac{1}{2}\left(\frac{\Delta \mathrm{r}}{\sigma_{r}}\right)^{2 m}\right],
$$

na qual $\Delta r^{2}=\Delta x^{2}+\Delta y^{2}, \Delta x=\left(x-x_{0}\right), \Delta y=\left(y-y_{0}\right)$ e $(\mathrm{x}, \mathrm{y})=\left(x_{0}, y_{0}\right)$ é o ponto central de excitação. O parâmetro $m$ controla o grau de variação do perfil nas bordas. Para $m=1$, tem-se a função gaussiana usual. O parâmetro $\sigma_{r}$, que no caso da gaussiana comum é o valor RMS, pode ser considerado o raio da supergaussiana.

Os valores utilizados são: $A_{0}=184 \mathrm{MV} / \mathrm{m} ; m=3 ; \sigma_{r}=12,5 \mu \mathrm{m}$ (ou diâmetro de $25 \mu \mathrm{m})$. Na Fig. 26 é traçado um esboço da simulação realizada. As Fig. 27-Fig. 33 ilustram a evolução do feixe óptico incidindo em uma interface com o ar para ângulos de incidência de 2,3 e $4^{\mathrm{o}}$, respectivamente.

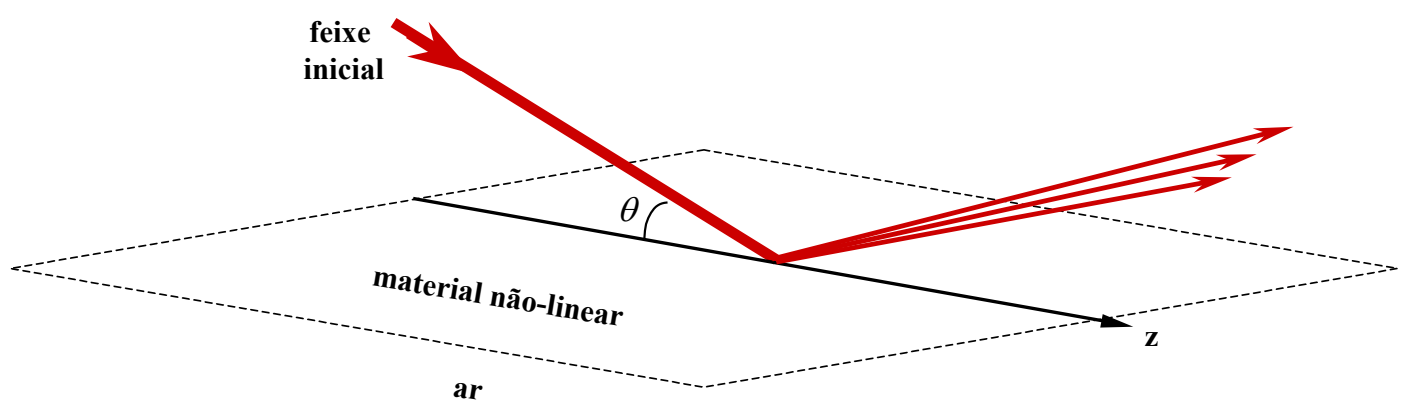

Fig. 26 - Esboço da simulação realizada no meio não-linear com saturação, onde um feixe óptico é totalmente refletido após incidir na interface com o ar.

As simulações mostram uma grande semelhança entre a dinâmica dos fluidos e a interferência de condensados de luz em contornos ou descontinuidades localizadas. O efeito observado pode ser imaginado como se os condensados de luz apresentassem algum tipo de "tensão superficial", analogamente ao que ocorre com gotas líquidas, conforme foi interpretado por Michinel et al. [59]. Os resultados obtidos são concordantes ao ilustrado em [59], apesar de alguns detalhes sobre a configuração inicial do pulso (tais como posição e ângulo de propagação) não serem fornecidas nesta referência para possibilitar uma comparação mais rigorosa. Foram 
considerados ângulos de incidência dentro do limite paraxial do formalismo, o que é suficiente para observar o efeito que o aumento deste parâmetro acarreta sobre o condensado óptico incidente. Como pode ser visto nestas simulações, o aumento do ângulo de incidência provoca o nascimento de novos condensados como previsto por Snyder et al. [61]. Como complemento às figuras mostradas, as tabelas a seguir listam as relações de amplitude entre as componentes minoritárias e a majoritária de campo elétrico, para cada ângulo de incidência na interface com o ar, observados no decorrer da propagação. Observe na TABELA 9 que em $\mathrm{z}=2,5 \mathrm{~mm}$ o condensado é afetado devido à colisão, o que justifica o maior desvio de amplitude neste ponto. $\mathrm{O}$ mesmo pode ser observado para na e para $\mathrm{z}=1,8 \mathrm{~mm}$ na TABELA 10 e para $\mathrm{z}=1,4$ $m m$ na TABELA 11.

A eficiência numérica no caso de problemas não-lineares é inferior à de um problema linear apresentando dimensionamento equivalente, uma vez que no caso não-linear esta eficiência está relacionada ao número de iterações necessárias para a convergência das componentes transversais de campo elétrico e da permissividade dielétrica. No caso da simulação caracterizada pelas Figs. 27-29, o tempo de processamento foi de aproximadamente 269 minutos, utilizando um processador AMD Athlon ${ }^{\mathrm{TM}}$ XP 2600 de 2,08 GHz de clock. Observou-se que o número máximo de iterações necessárias para a convergência dos campos foi três para uma tolerância $\delta_{t o l}=10^{-8} \mathrm{em}(4.39)$ e $(4.48)$.

TABELA 9 - Relação entre as componentes minoritárias e a majoritária para incidência a $2^{\circ}$ na interface com o ar (v. Fig. 27 a Fig. 29)

\begin{tabular}{|c|c|c|c|}
\hline $\boldsymbol{z}(\boldsymbol{m m})$ & $\boldsymbol{E}_{\boldsymbol{y}} / \boldsymbol{E}_{\boldsymbol{x}}$ & $\boldsymbol{E}_{z} / \boldsymbol{E}_{\boldsymbol{x}}$ & $\boldsymbol{E}_{\boldsymbol{x}}(\boldsymbol{M V} / \boldsymbol{m})$ \\
\hline 0,0 & 0,0 & 0,02247888538043 & 184,0 \\
\hline 2,0 & 0,00239583368708 & 0,02552264619838 & 183,333133 \\
\hline 2,5 & 0,00269896839066 & 0,03981467443694 & 204,291191 \\
\hline 3,0 & 0,00205100223567 & 0,02705935182812 & 179,669967 \\
\hline 3,5 & 0,00219458194625 & 0,03015885431857 & 184,784841 \\
\hline 4,0 & 0,00192088971160 & 0,03135441441549 & 186,109611 \\
\hline
\end{tabular}


TABELA 10 - Relação entre as componentes minoritárias e a majoritária para incidência a $3^{\circ}$ na interface com o ar (v. Fig. 30 a Fig. 32)

\begin{tabular}{|c|c|c|c|}
\hline $\boldsymbol{z}(\boldsymbol{m} \boldsymbol{m})$ & $\boldsymbol{E}_{\boldsymbol{y}} / \boldsymbol{E}_{\boldsymbol{x}}$ & $\boldsymbol{E}_{\boldsymbol{z}} / \boldsymbol{E}_{\boldsymbol{x}}$ & $\boldsymbol{E}_{\boldsymbol{x}}(\boldsymbol{M V} / \boldsymbol{m})$ \\
\hline 0,0 & 0,0 & 0,02245060880435 & 184,0 \\
\hline 1,8 & 0,00304008677610 & 0,05441579710294 & 200,549228 \\
\hline 2,2 & 0,00277843428841 & 0,04390659973250 & 178,642786 \\
\hline 2,6 & 0,00290657752523 & 0,04057077224163 & 180,568627 \\
\hline 3,0 & 0,00297812511523 & 0,03976008370532 & 187,834178 \\
\hline 3,2 & 0,00258729563189 & 0,04686154102518 & 196,102882 \\
\hline
\end{tabular}

TABELA 11 - Relação entre as componentes minoritárias e a majoritária para incidência a $4^{\circ}$ na interface com o ar (v. Fig. 33 a Fig. 35)

\begin{tabular}{|c|c|c|c|}
\hline $\mathbf{z}(\boldsymbol{\mu m})$ & $\boldsymbol{E}_{\boldsymbol{y}} / \boldsymbol{E}_{\boldsymbol{x}}$ & $\boldsymbol{E}_{\boldsymbol{z}} / \boldsymbol{E}_{\boldsymbol{x}}$ & $\boldsymbol{E}_{\boldsymbol{x}}(\mathbf{M V} / \mathbf{m})$ \\
\hline 0 & 0,0 & 0,02238460521739 & 184,0 \\
\hline 1,4 & 0,00230977537011 & 0,06205562583590 & 205,222552 \\
\hline 1,8 & 0,00310698575279 & 0,05611844469706 & 176,738533 \\
\hline 2,2 & 0,00314838646251 & 0,05667775714461 & 180,041835 \\
\hline 2,7 & 0,00155666174780 & 0,03574067378613 & 181,762157 \\
\hline 3,2 & 0,00183928018831 & 0,03171783389251 & 180,504542 \\
\hline
\end{tabular}



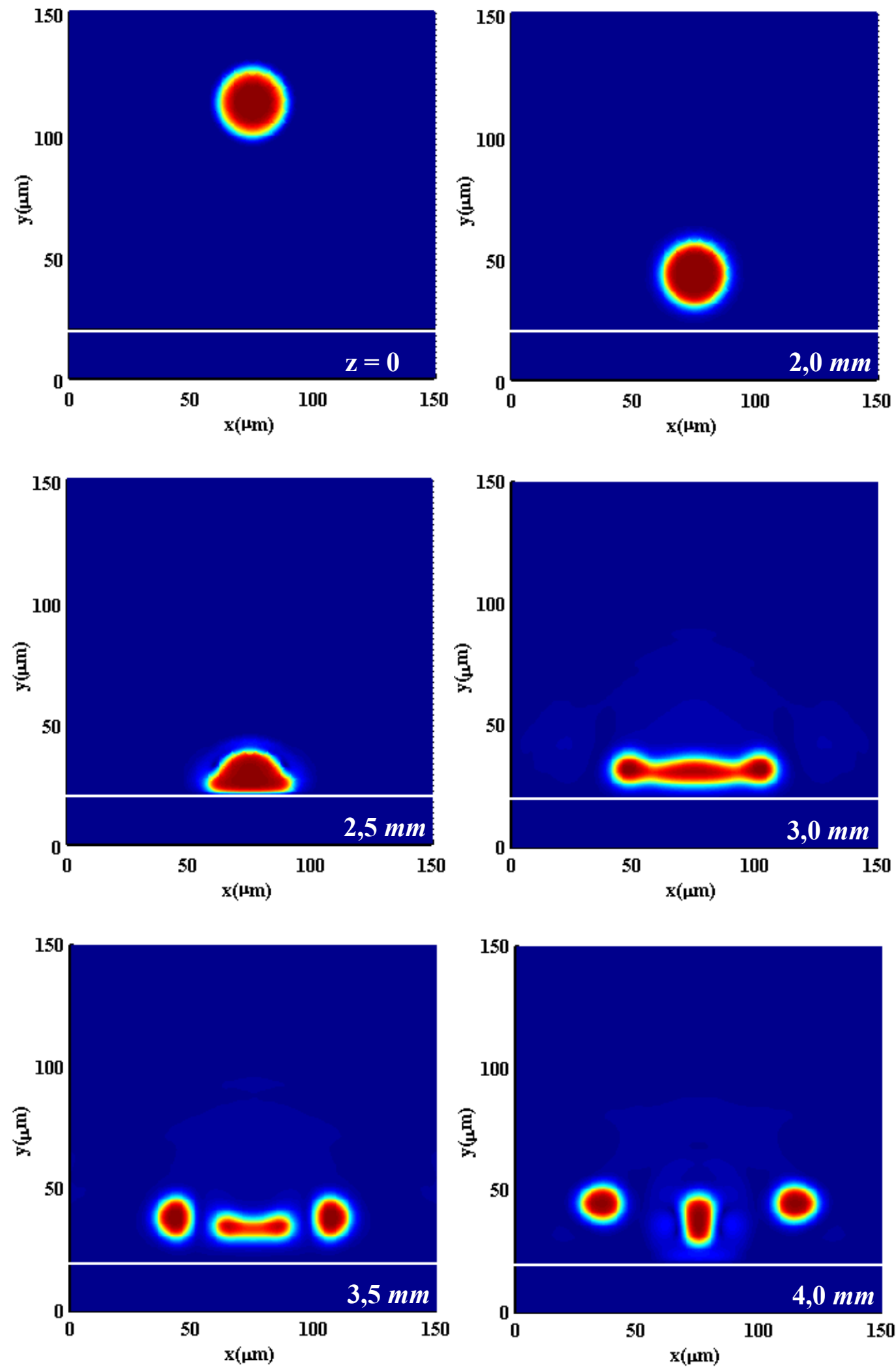

Fig. 27 - Choque de um feixe óptico condensado em uma interface com o ar para um ângulo de incidência de $2^{\circ}$, componente $\mathrm{E}_{\mathrm{x}}$. 

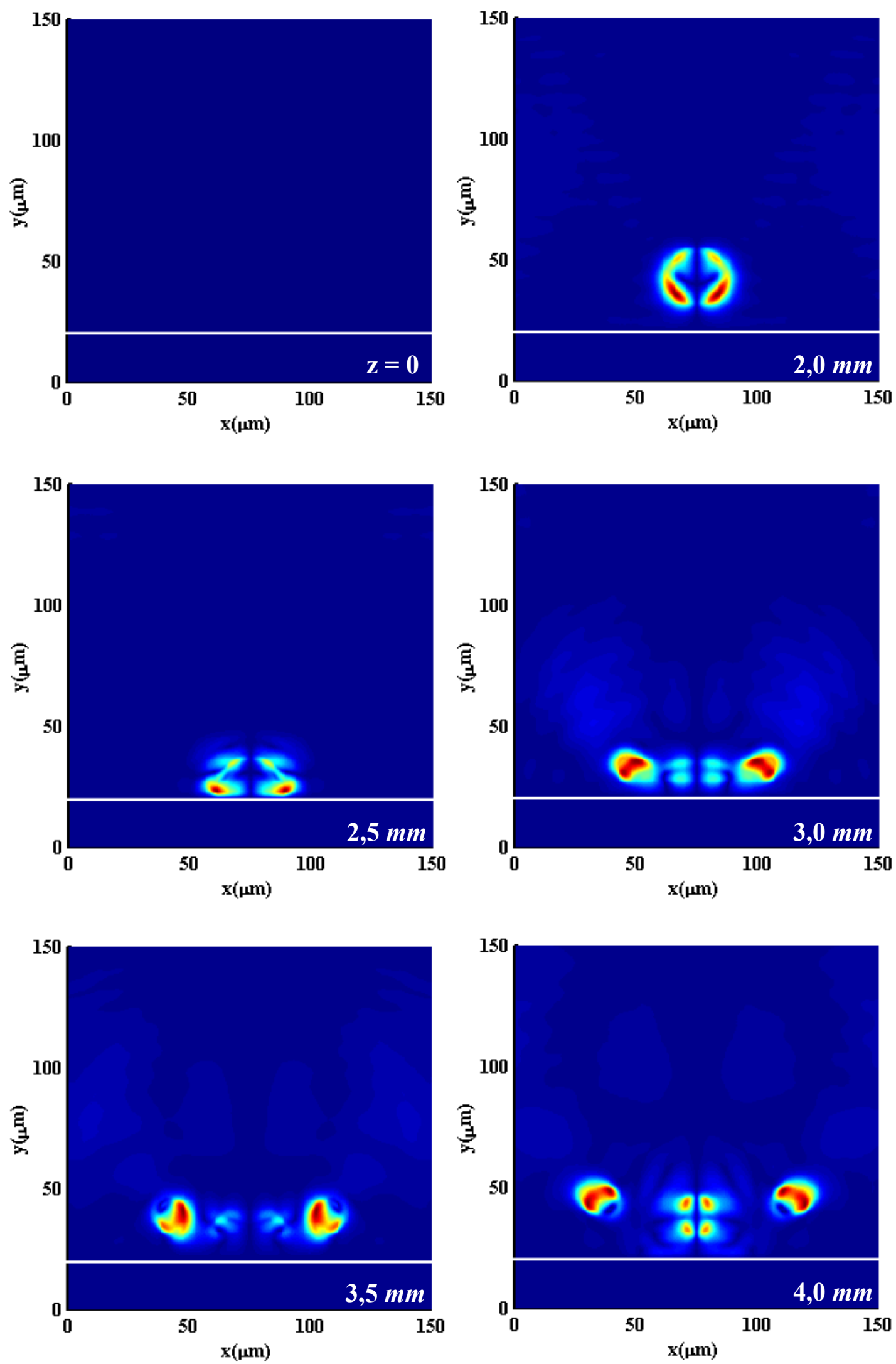

Fig. 28 - Observação da evolução da componente $E_{y}$ referente ao mesmo exemplo da Fig. 27. 

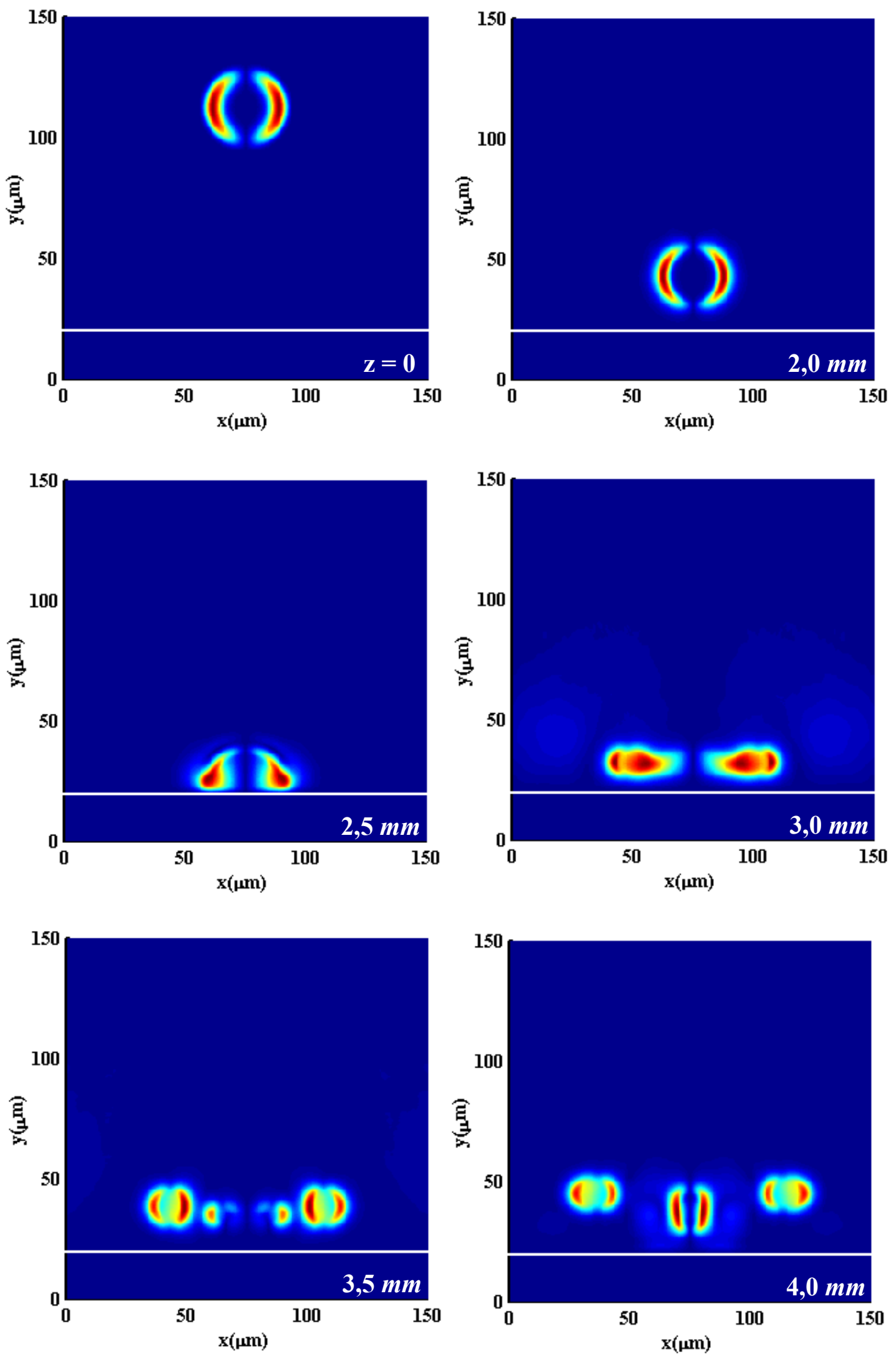

Fig. 29 - Observação da evolução da componente $E_{z}$ referente ao mesmo exemplo da Fig. 27. 

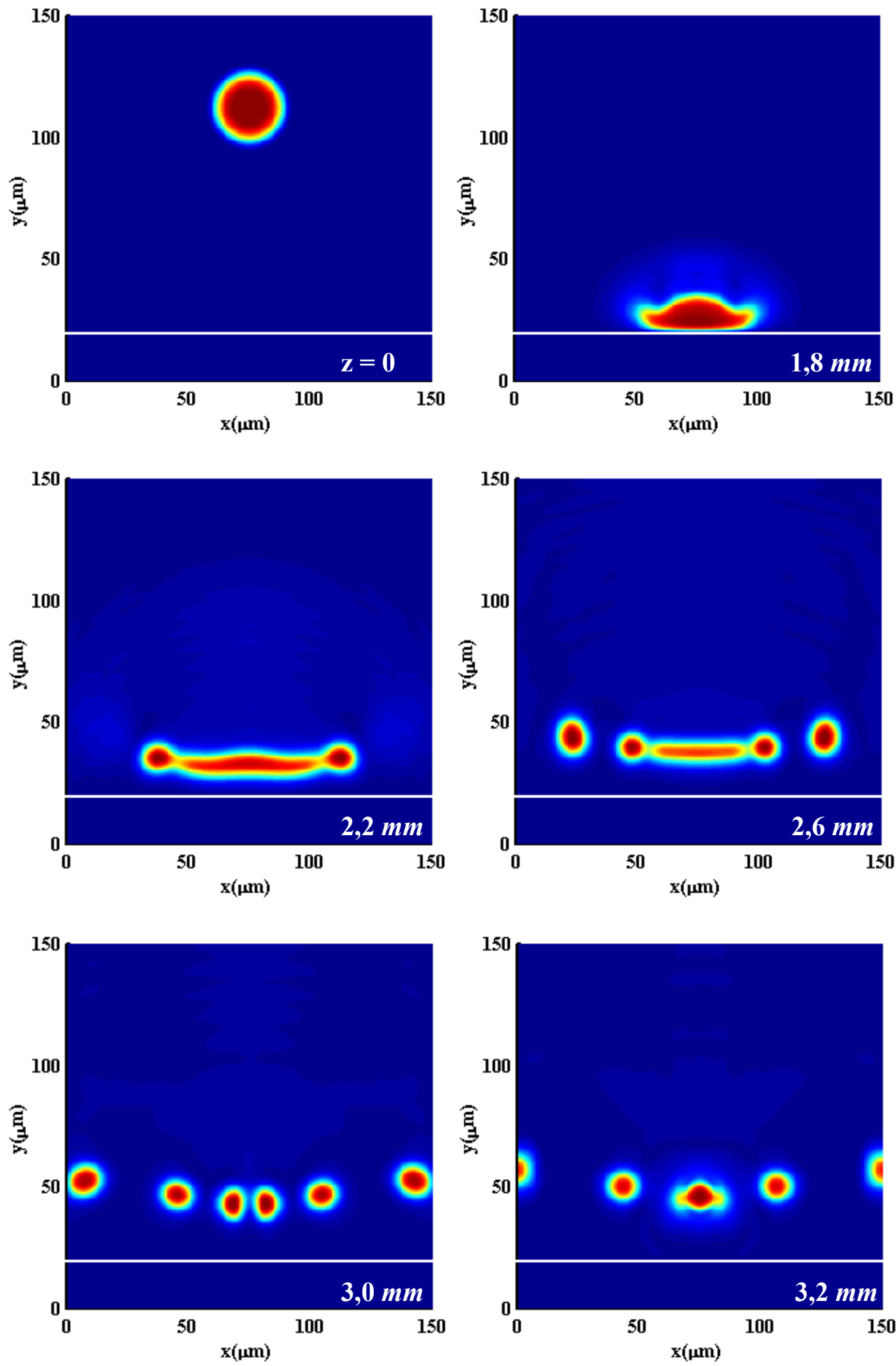

Fig. 30 - Componente dominante $E_{x}$ referente ao choque de um feixe óptico condensado em uma interface com o ar para um ângulo de incidência de $3^{\circ}$. 

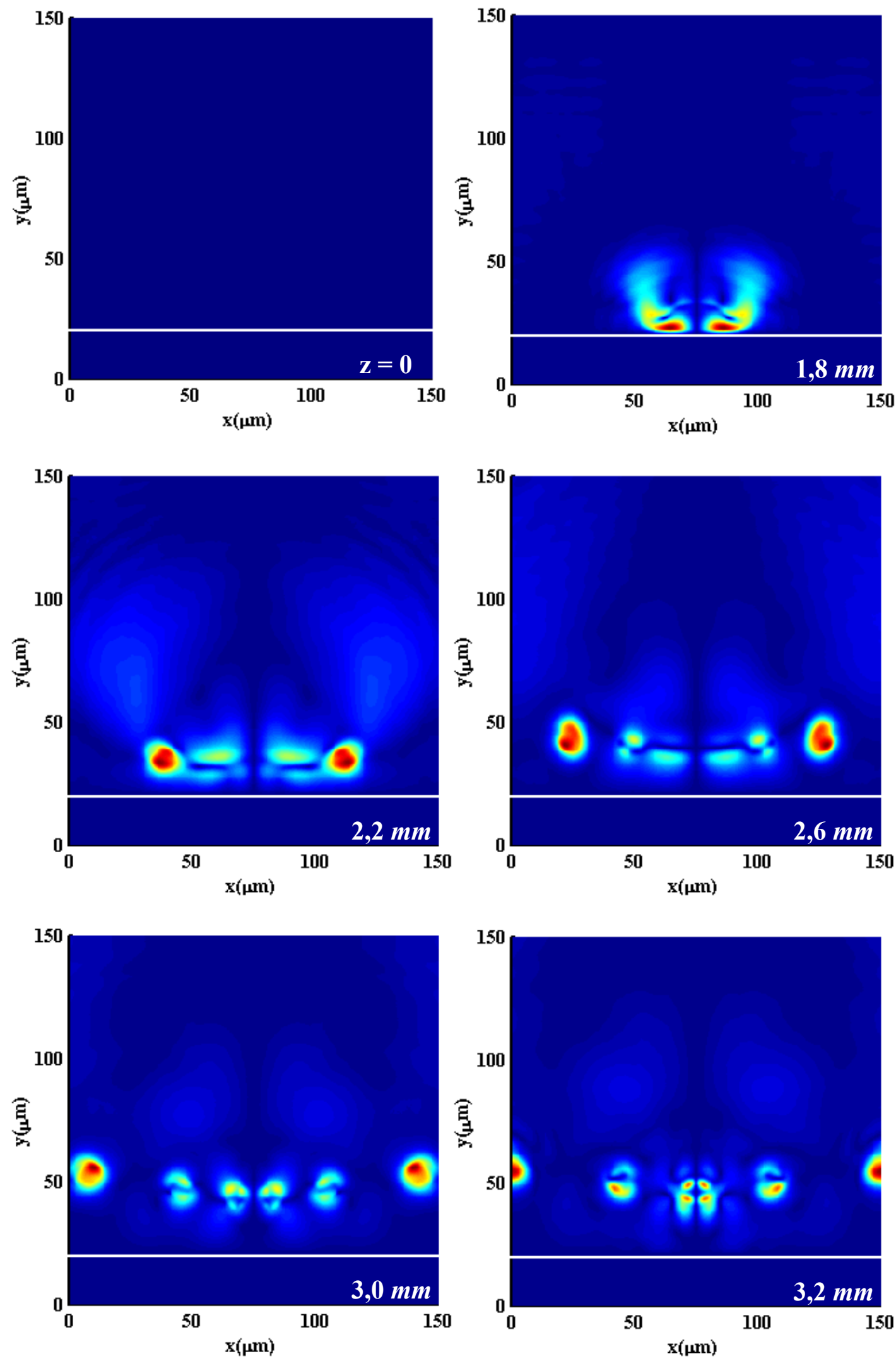

Fig. 31 - Componente $E_{y}$ referente ao choque de um feixe óptico condensado em uma interface com o ar para um ângulo de incidência de $3^{\circ}$. 

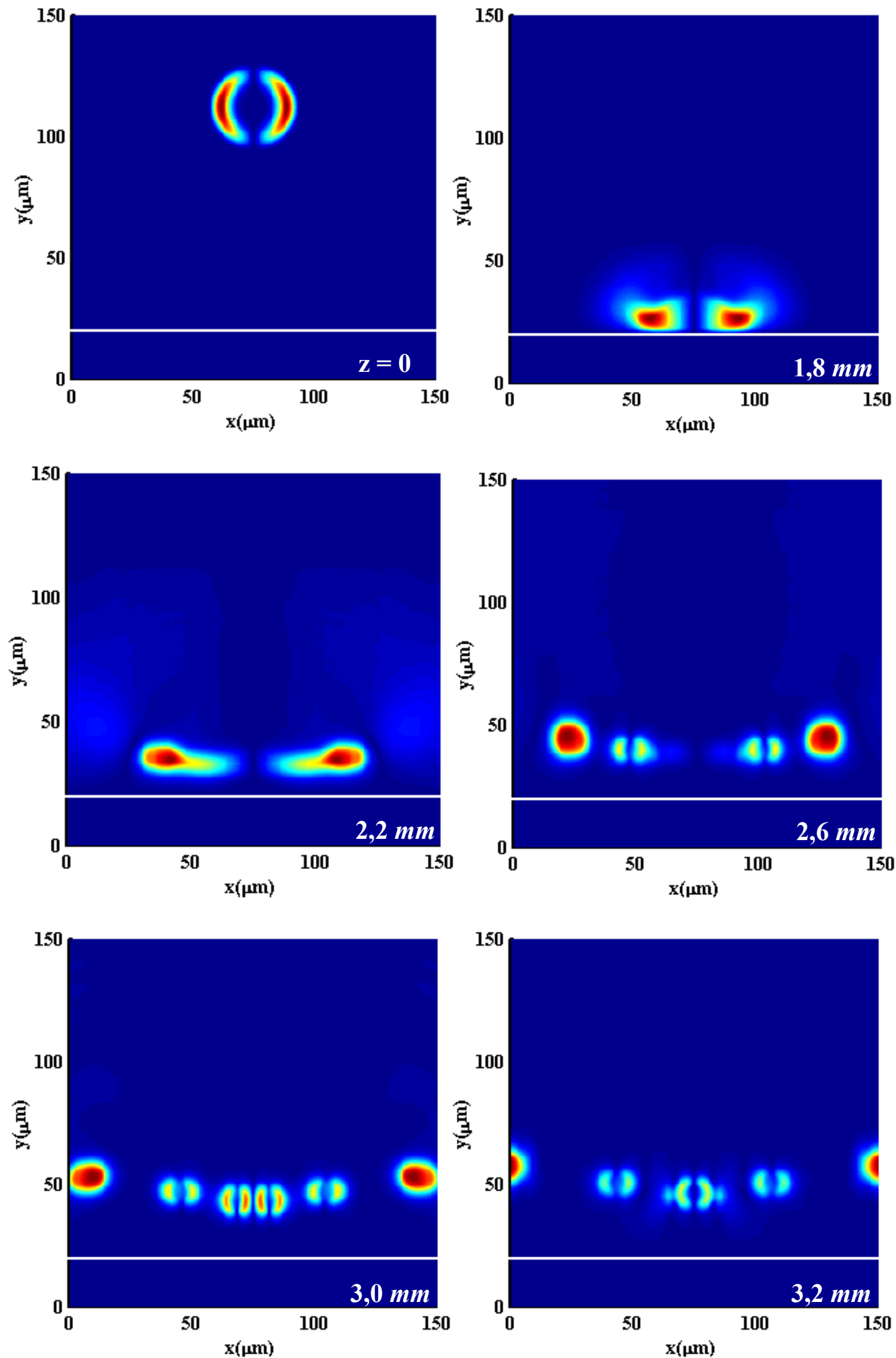

Fig. 32 - Componente $E_{z}$ referente ao choque de um feixe óptico condensado em uma interface com o ar para um ângulo de incidência de $3^{\circ}$. 

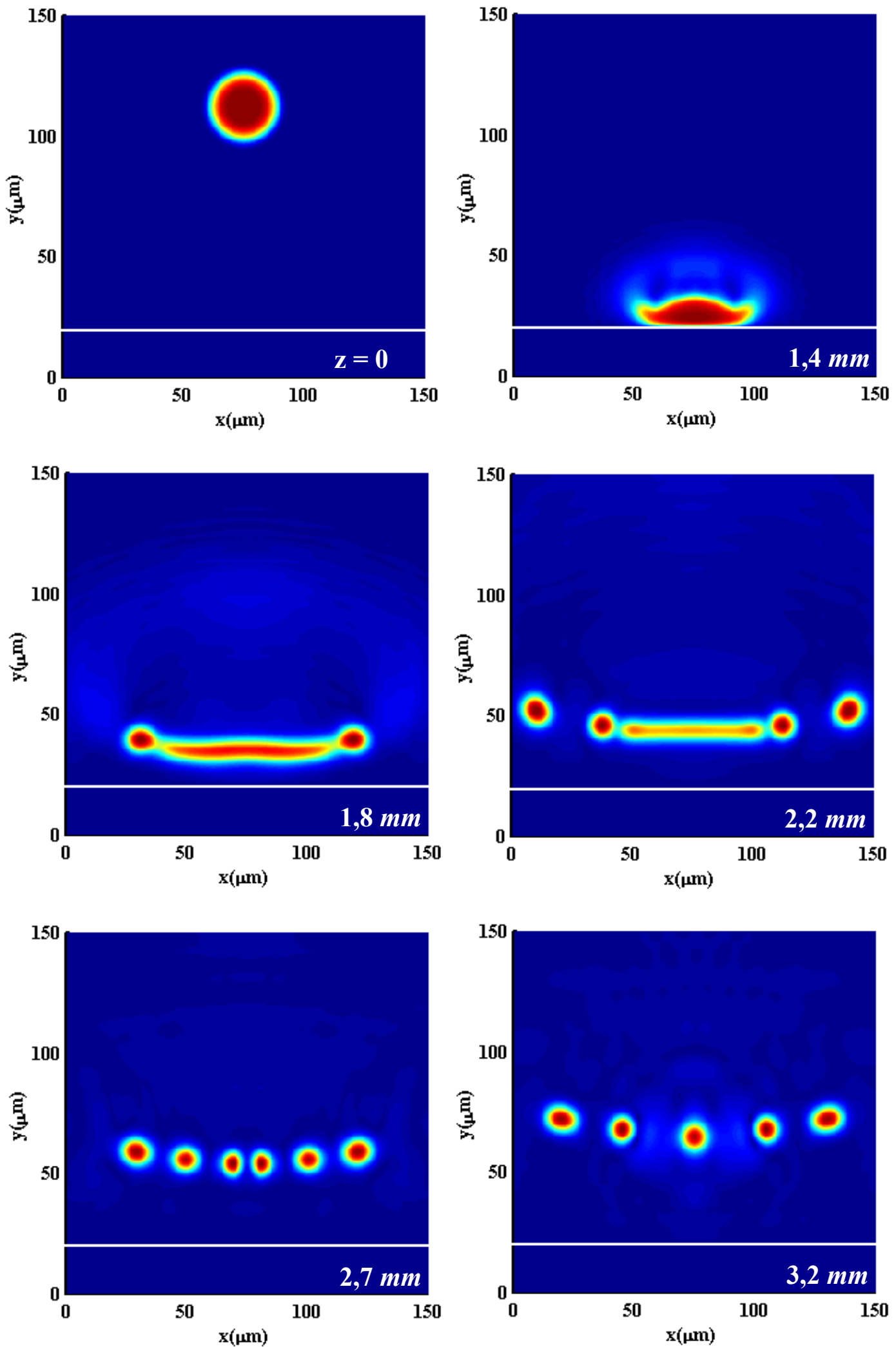

Fig. 33 - Componente dominante $E_{x}$ referente ao choque de um feixe óptico condensado em uma interface com o ar para um ângulo de incidência de $4^{\circ}$. 

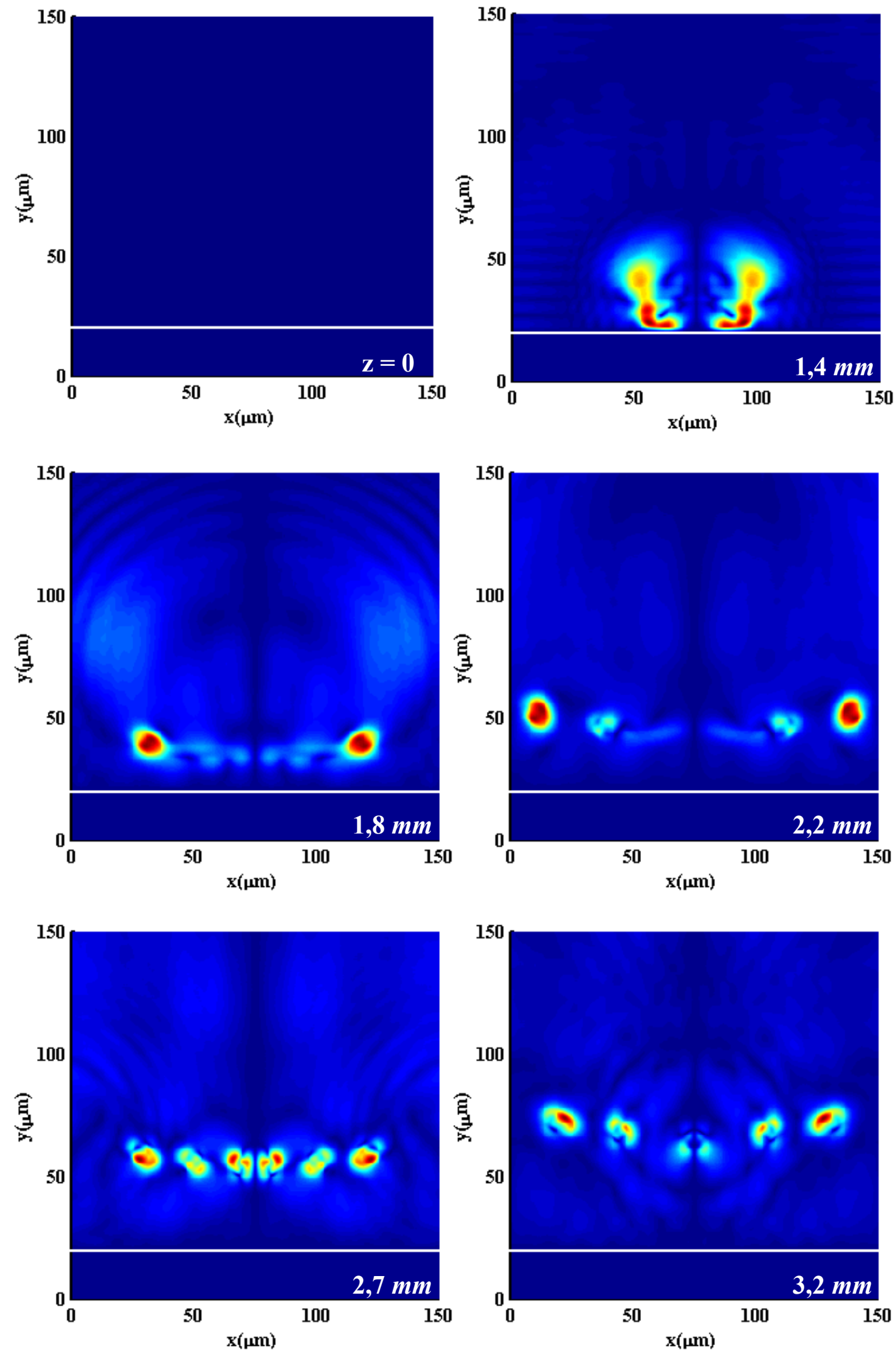

Fig. 34 - Componente $E_{y}$ referente ao choque de um feixe óptico condensado em uma interface com o ar para um ângulo de incidência de $4^{\circ}$. 

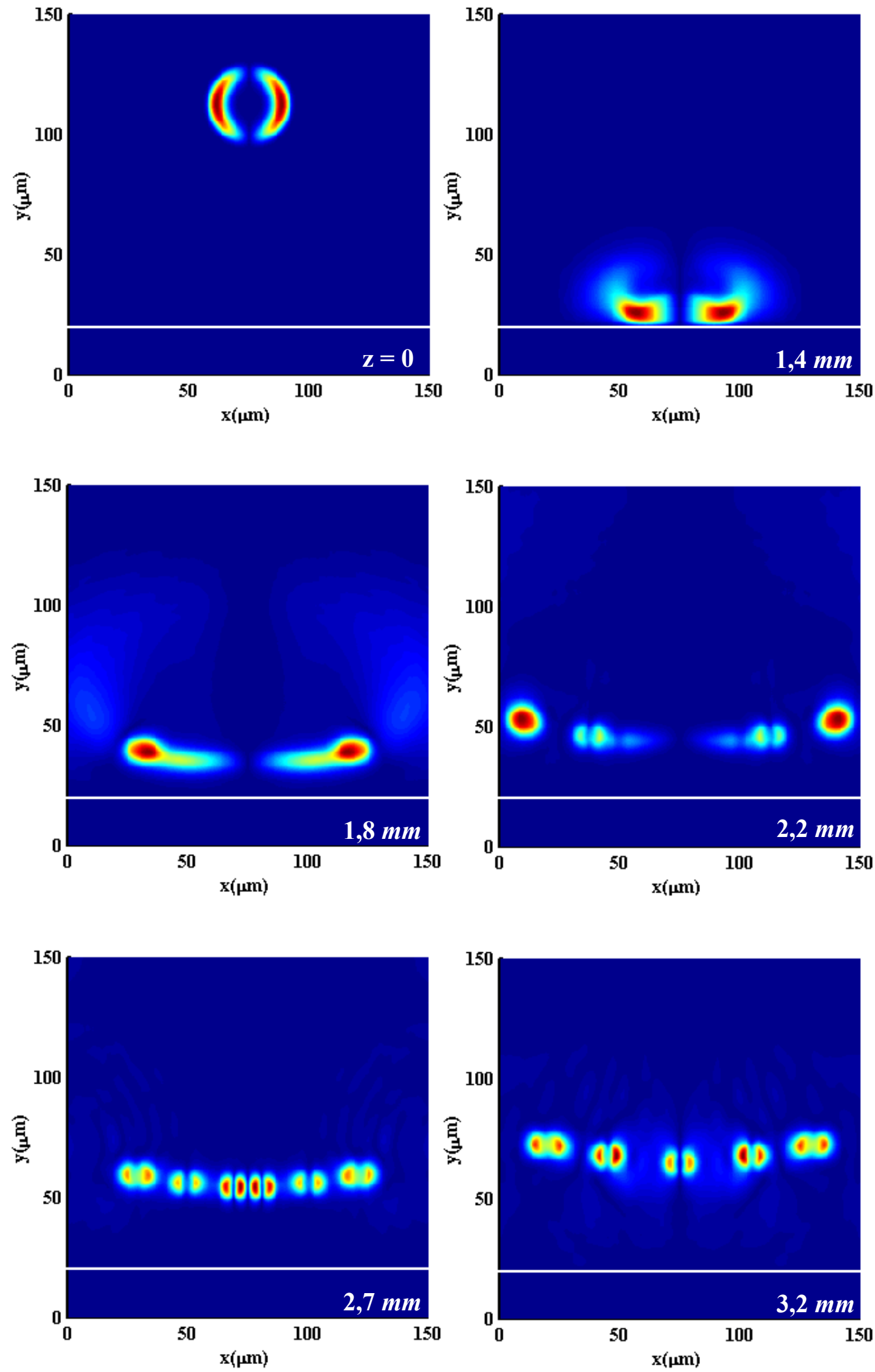

Fig. 35 - Componente $E_{z}$ referente ao choque de um feixe óptico condensado em uma interface com o ar para um ângulo de incidência de $4^{\circ}$. 


\subsubsection{Colisão entre dois feixes ópticos}

Nesta subseção são mostradas as distribuições de campo para a componente $E_{x}$ para a situação de "colisão" entre dois feixes ópticos que se propagam no meio do tipo Kerr com saturação. A colisão entre sólitons pode produzir efeitos muito interessantes, tais como troca de energia, fusão, aniquilação, ou até mesmo o nascimento de novos sólitons [61]-[63]. A colisão entre sólitons pode ser classificada como 1) elástica, quando há pouca (ou nenhuma) perda de energia ou mudança de formato, 2) quase-elástica, quando eles se separam após a colisão e emergem com aplitudes menores, e 3) inelástica, quando eles se separam após a colisão com uma conseqüente perda de energia [64]-[65]. Portanto, em uma colisão inelástica haverá emissão de radiação. O comportamento inelástico observado por este tipo de colisão é devido à não-integrabilidade da equação de Helmholtz tridimensional.

A Fig. 36 ilustra o caso em que os feixes que colidem estão em fase, enquanto que a Fig. 39 ilustra o choque para o caso de uma defasagem de $180^{\circ}$ entre os pulsos. As TABELAS 12 e 13 listam as razões entre os valores de pico das componentes minoritárias e da majoritária ao longo da propagação. A observação dos resultados obtidos permite concluir que a fase relativa é outro parâmetro que afeta a interação entre os pulsos ópticos em meios não-lineares, provocando certa atração ou repulsão, analogamente ao que ocorre entre cargas elétricas.

No caso de colisão em fase, as amplitudes dos feixes se adicionam na medida em que se aproximam provocando a atração destes devido ao aumento do índice de refração nas bordas das supergaussianas na posição onde ocorre o encontro, provocando a união dos feixes. Entretanto, um aumento ainda maior da intensidade de campo elétrico provoca um decréscimo considerável do índice de refração no centro da colisão logo após o encontro, provocando a liberação de feixes lateralmente (direção na qual a "inércia" é vencida mais facilmente), de modo que a potência óptica remanescente produz uma distribuição de índice de refração estável, resultando em um feixe supergaussiano central, propagando coincidentemente ao eixo $z$.

No caso de colisão com defasagem de $180^{\circ}$, as amplitudes dos feixes se cancelam na medida em que se aproximam, provocando a repulsão destes devido à 
diminuição abrupta do índice de refração nas bordas das supergaussianas, na posição onde ocorre o encontro. A partir deste ponto a análise é análoga à colisão de um dos pulsos isolados em uma interface (compare a Fig. 39 com a Fig. 30)

Os fenômenos observados podem ser imaginados como uma operação "NXOR" lógica. Suponha que exista um fotodetector situado longitudinalmente a uma certa distância do ponto de excitação (em $\mathrm{z}=5,4 \mathrm{~mm}$, por exemplo) e posicionado transversalmente entre os dois feixes. Estes feixes em fase podem ser considerados como bits "1", fazendo o sinal atingir o detector com amplitude da mesma ordem de um dos feixes isolados, devido ao efeito de saturação. A inversão de um dos bits pode ser representada pela defasagem de $180^{\circ}$ de um dos feixes, que após serem repelidos poderiam ser identificados por detectores verticalmente afastados do detector central. A minimização da geração de feixes espúrios após a colisão poderia ser controlada por meio dos diâmetros dos feixes iniciais e do ângulo de colisão. Interfaces ou falhas no material não-linear poderiam ser aplicadas com o objetivo de redirecionar o(s) feixe(s) de saída para a realização de novas operações com outros feixes. Entretanto, conforme comentado em [58], vários requisitos devem ser satisfeitos no projeto de portas lógicas eficientes. Estes requisitos são completitude lógica, três terminais, nível de referência para decisão (thresholding), cascateamento, ganho para compensar perdas, paralelismo, velocidade, pipelining, fan-in, insensibilidade à fase, baixo consumo de potência e facilidade de implementação.

Para atingir um baixo consumo de potência, o sóliton deve estar completamente confinado nas três dimensões. Isto descarta a aplicação de excitação de feixes $\mathrm{CW}$, como é o caso de sólitons espaciais unidimensionais e bidimensionais. Portanto, light bullets se tornam a melhor opção para um mínimo consumo de energia possível, além de proporcionar um melhor paralelismo, pois portas lógicas poderiam ser orientadas ao longo das três dimensões, elevando a integração do dispositivo. O requisito de insensibilidade à fase deve-se a necessidade de garantir a robustez do sistema extenso, o qual irá se expandir e contrair devido ao calor, vibração e tolerâncias de fabricação [58]. A interação entre pares de sólitons com polarizações ortogonais torna o sistema independente à fase. Contudo, o efeito de repulsão entre sólitons não poderia ser mais explorado. Com relação ao quesito 
facilidade de implementação, portas lógicas baseadas em detecção espacial (posição do sóliton de saída) são mais simples do que as baseadas em detecção temporal (detecção coincidente ultra-rápida do sóliton de saída resultante da interação de par de sólitons com velocidades de grupos diferentes).

De acordo com [58], a configuração de porta lógica óptica que melhor satisfaz aos requisitos listados é baseada na interação de light bullets ortogonalmente polarizadas. Esta interação ocorreria por "captura" (dragging ou trapping), que consiste em uma configuração na qual os sólitons são inicialmente coincidentes, porém direcionados em ângulos espaciais diferentes. O valor lógico resultante seria determinado pela passagem ou não do sóliton de saída através de uma trajetória especificada. Ao contrário de outras implementações, na captura é simples interagir um sóliton de bombeamento com um sóliton de sinal enfraquecido, resultando na restauração do sinal lógico (ganho).

TABELA 12 - Relação entre as componentes minoritárias e a majoritária para colisão entre condensados ópticos em fase (v. Fig. 36 a Fig. 38)

\begin{tabular}{|c|c|c|c|}
\hline $\mathbf{z}(\boldsymbol{\mu m})$ & $\boldsymbol{E}_{\boldsymbol{y}} / \boldsymbol{E}_{\boldsymbol{x}}$ & $\boldsymbol{E}_{\boldsymbol{z}} / \boldsymbol{E}_{\boldsymbol{x}}$ & $\boldsymbol{E}_{\boldsymbol{x}}(\mathbf{M V} / \mathbf{m})$ \\
\hline 0 & 0 & 0,02245072259118 & 184,0 \\
\hline 400 & 0,00177539221692 & 0,02476629156104 & 184,040252 \\
\hline 800 & 0,00212684171563 & 0,06085168896794 & 188,280424 \\
\hline 1200 & 0,00186892022630 & 0,04107455906872 & 192,023348 \\
\hline 2200 & 0,00151480067589 & 0,04341062085813 & 182,887256 \\
\hline 5400 & 0,00224780674943 & 0,02877278883039 & 190,714757 \\
\hline
\end{tabular}

TABELA 13 - Relação entre as componentes minoritárias e a majoritária para colisão entre condensados ópticos defasados de $180^{\circ}$ (v. Fig. 39 a Fig. 41)

\begin{tabular}{|c|c|c|c|}
\hline $\mathbf{z}(\boldsymbol{\mu m})$ & $\boldsymbol{E}_{\boldsymbol{y}} / \boldsymbol{E}_{\boldsymbol{x}}$ & $\boldsymbol{E}_{\boldsymbol{z}} / \boldsymbol{E}_{\boldsymbol{x}}$ & $\boldsymbol{E}_{\boldsymbol{x}}(\mathbf{M V} / \mathbf{m})$ \\
\hline 0 & 0 & 0,02602402418478 & 184,0 \\
\hline 400 & 0,00185610426260 & 0,02434011996272 & 184,564668 \\
\hline 800 & 0,00294394395267 & 0,05550455229679 & 185,227598 \\
\hline 1200 & 0,00267738165542 & 0,04474773729646 & 180,581213 \\
\hline 1900 & 0,00219719440309 & 0,04163823977369 & 182,629585 \\
\hline 2200 & 0,00267158191352 & 0,02827168247083 & 196,506848 \\
\hline
\end{tabular}



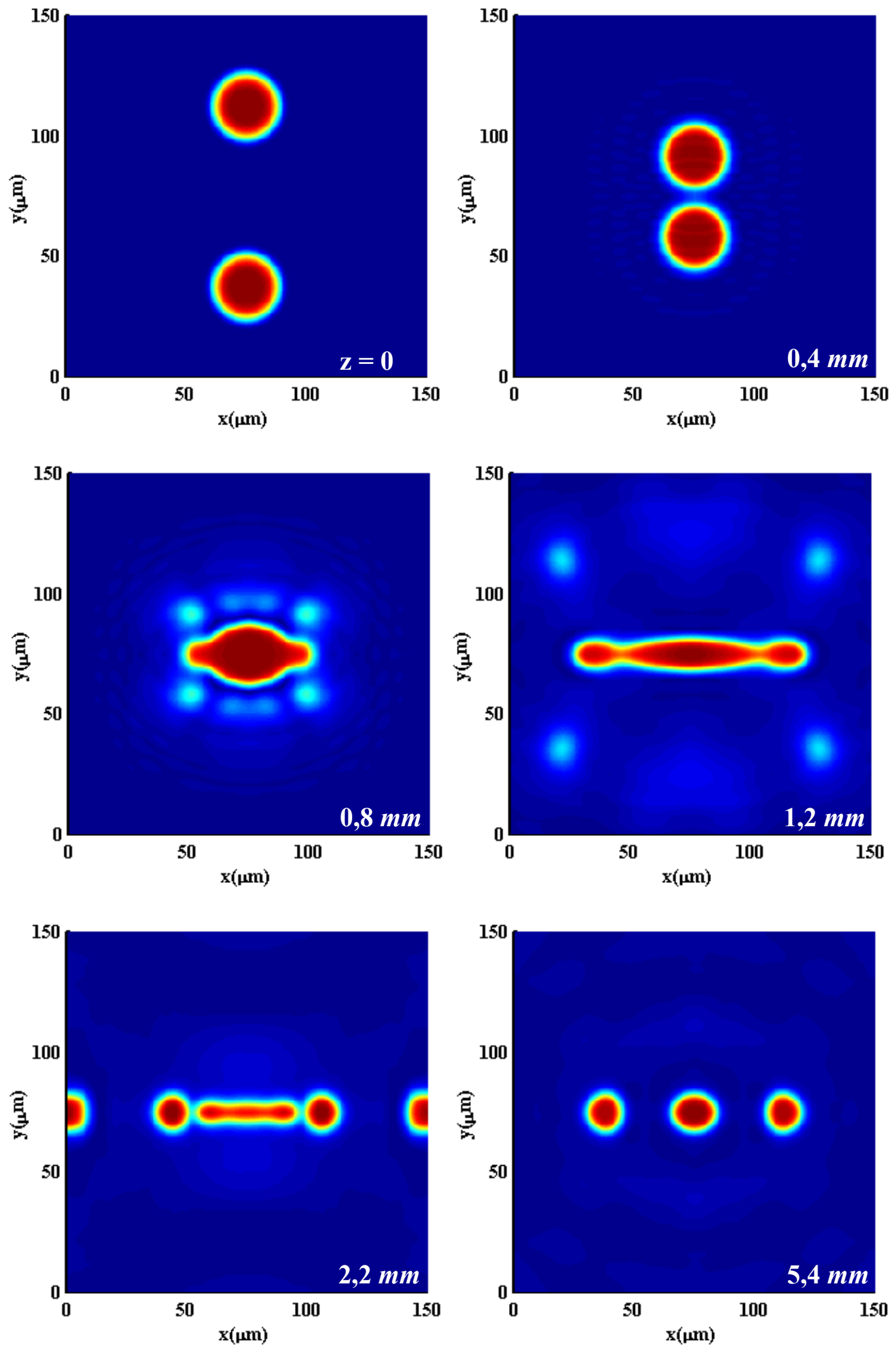

Fig. 36 - Componente dominante $E_{x}$ referente ao choque entre feixes ópticos em fase para um ângulo de propagação de $3^{\circ} \mathrm{em}$ relação ao plano horizontal. 

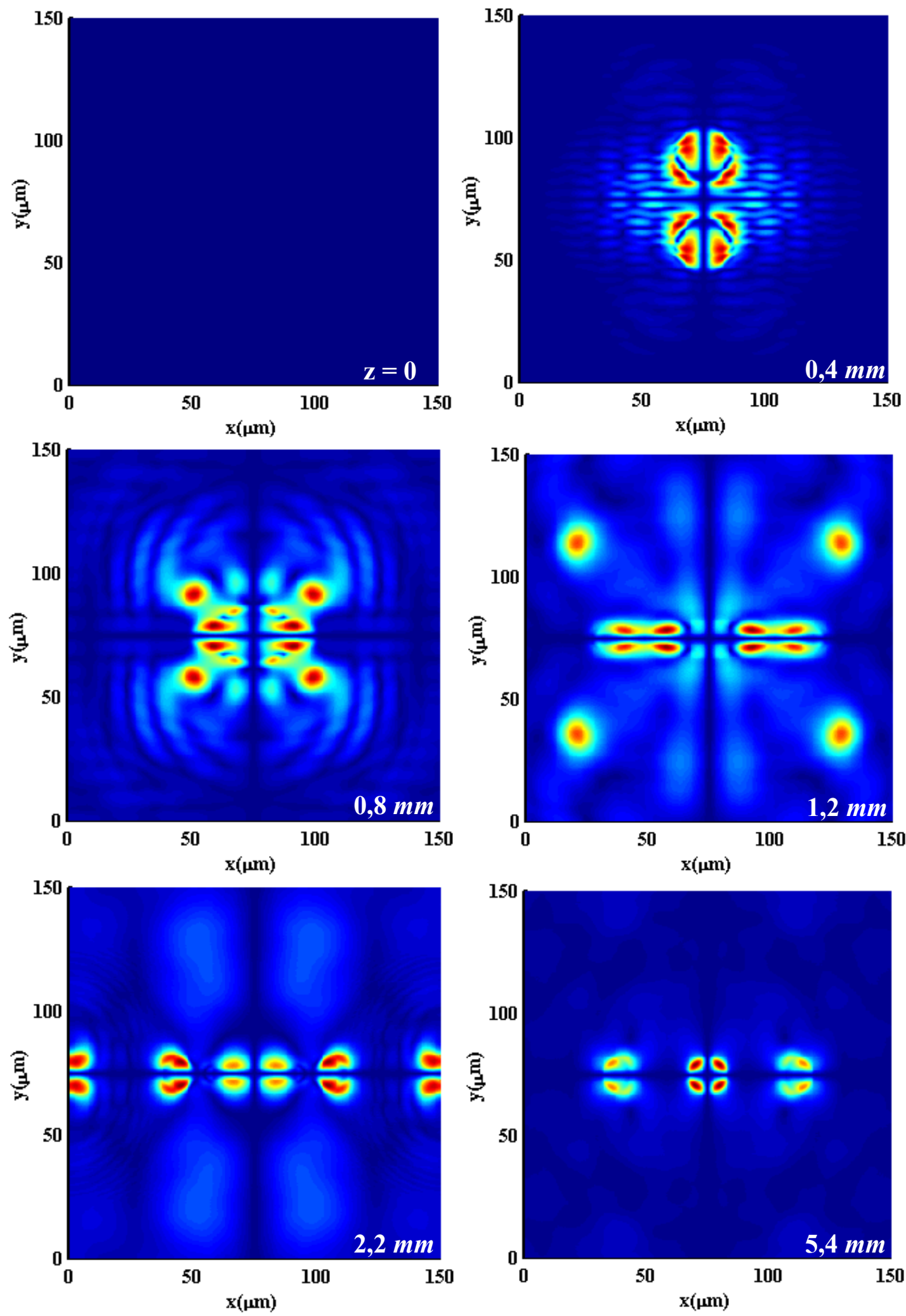

Fig. 37 - Componente $E_{y}$ referente ao choque entre feixes ópticos em fase para um ângulo de propagação de $3^{\circ}$ em relação ao plano horizontal. 

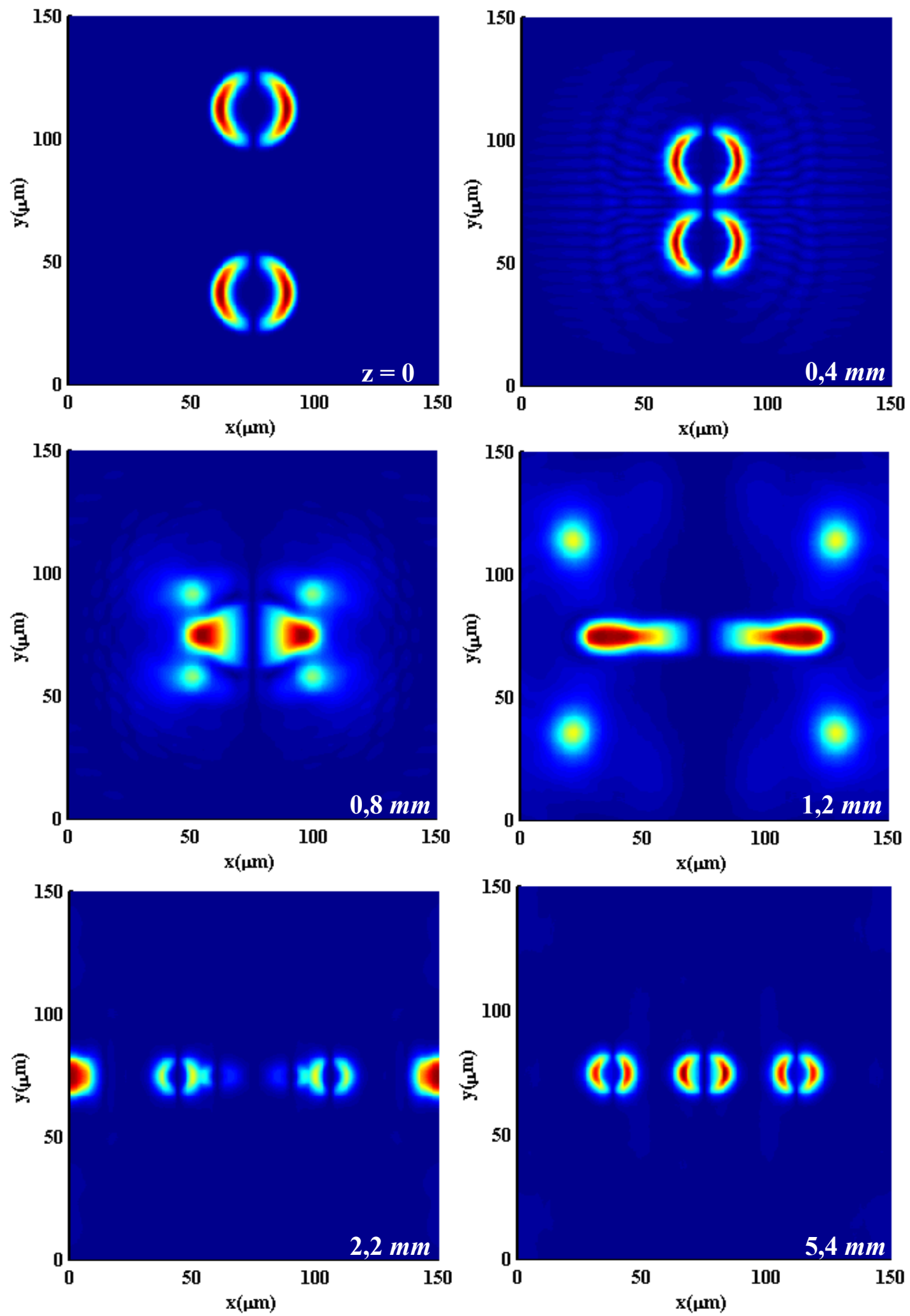

Fig. 38 - Componente $E_{z}$ referente ao choque entre feixes ópticos em fase para um ângulo de propagação de $3^{\circ}$ em relação ao plano horizontal. 

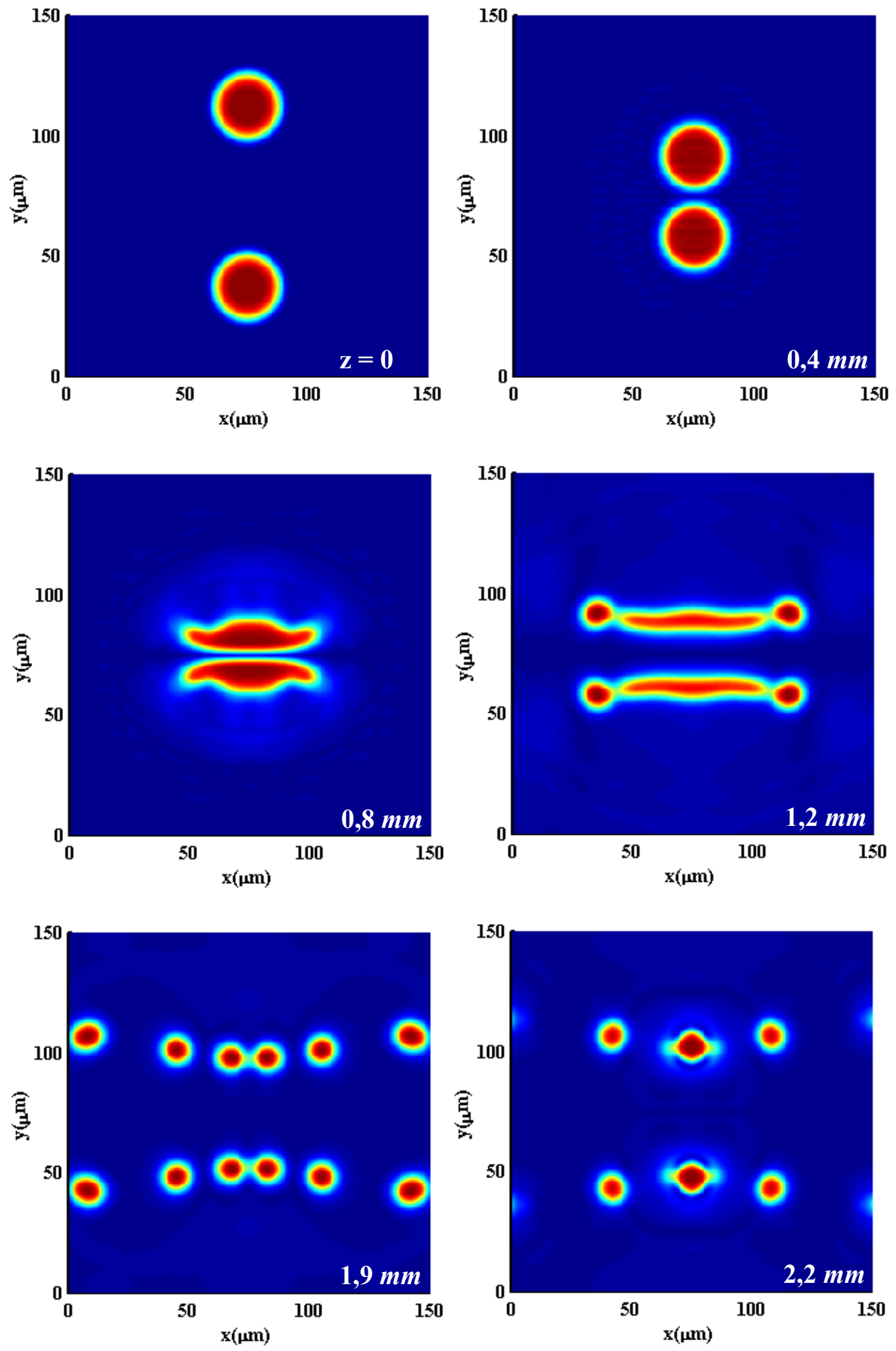

Fig. 39 - Componente dominante $E_{x}$ referente ao choque entre feixes ópticos defasados de $180^{\circ}$ para um ângulo de propagação de $3^{\circ}$ em relação ao plano horizontal. 

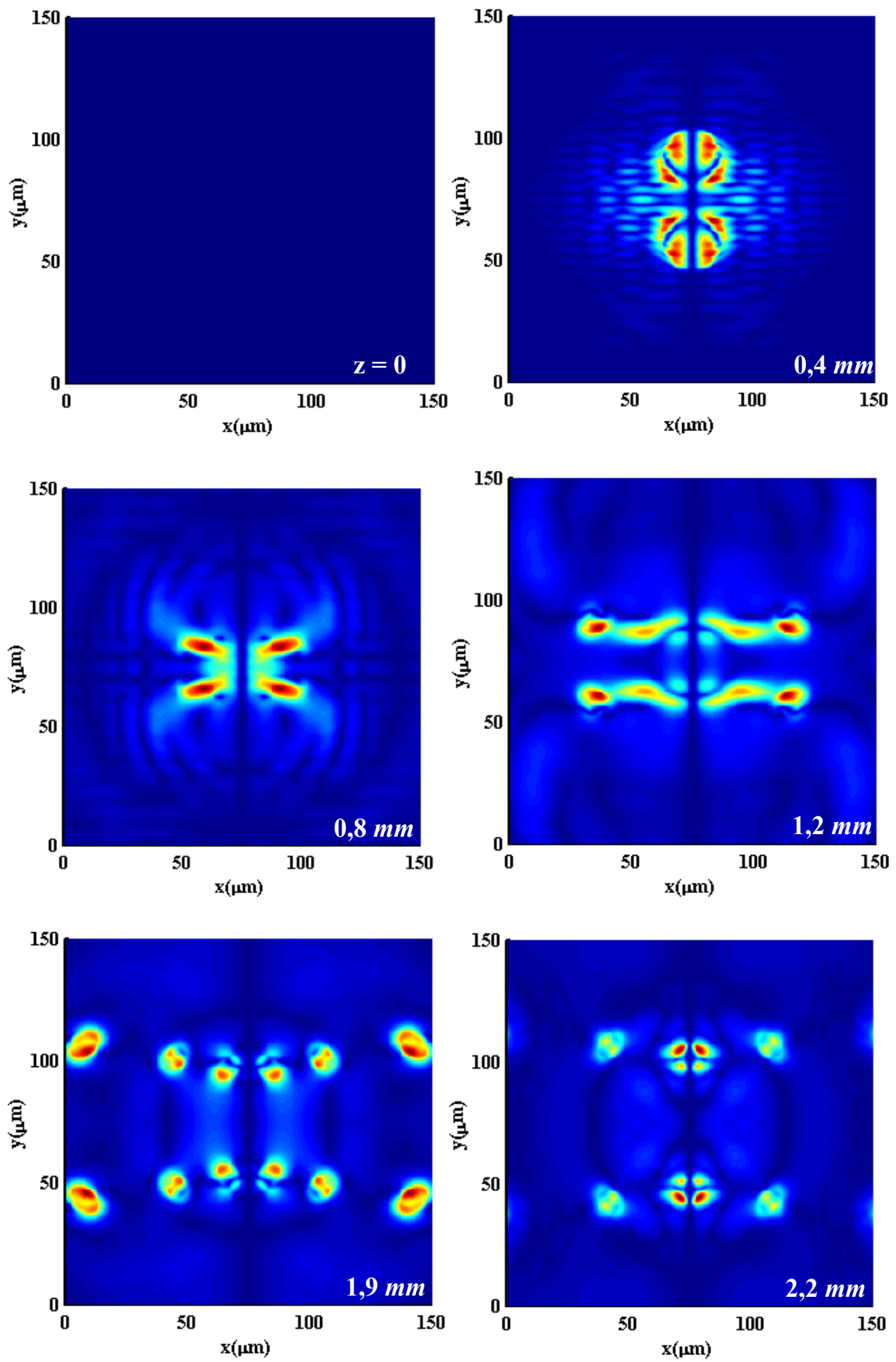

Fig. 40 - Componente $E_{y}$ referente ao choque entre feixes ópticos defasados de $180^{\circ}$ para um ângulo de propagação de $3^{\circ}$ em relação ao plano horizontal. 

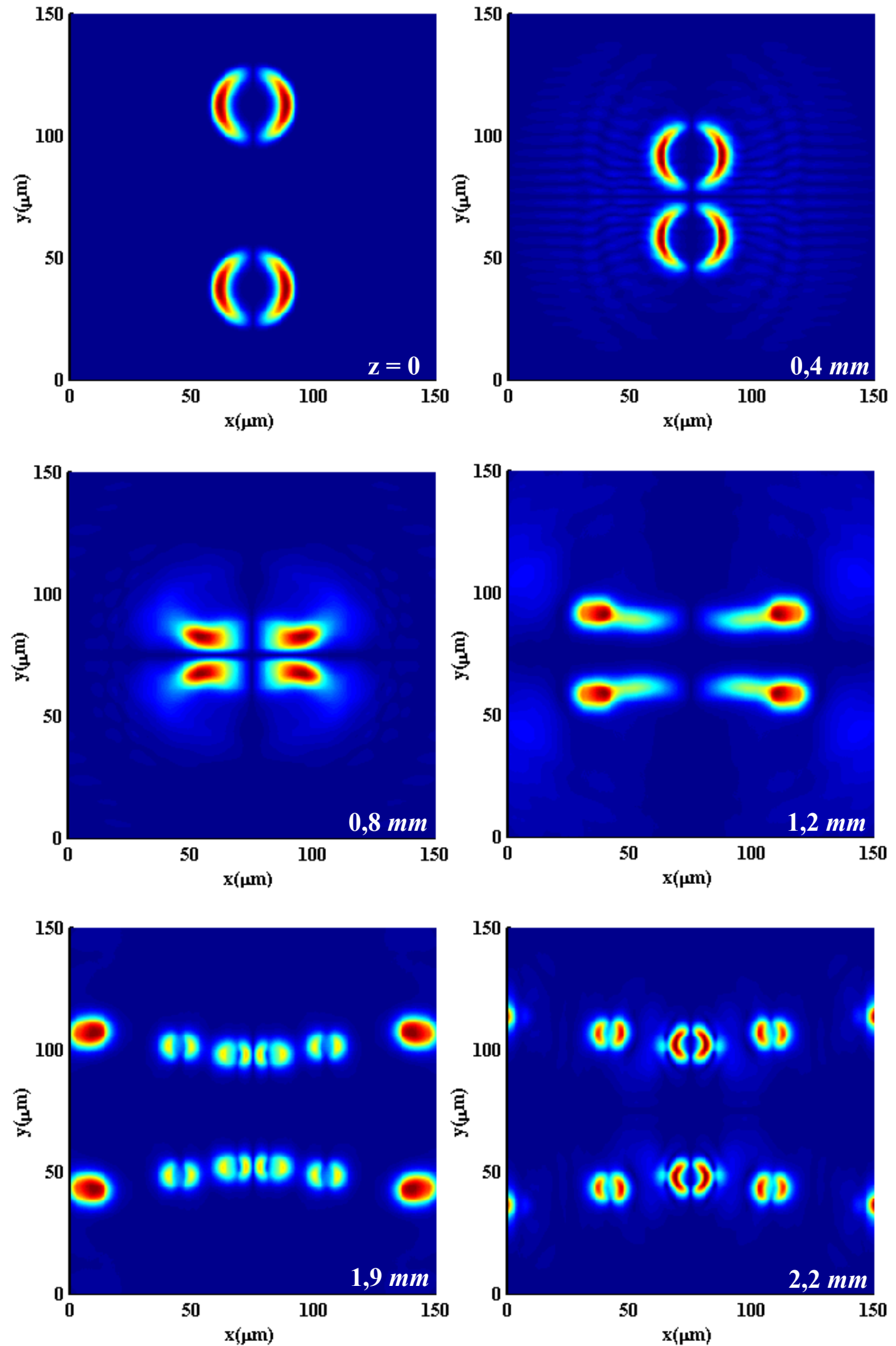

Fig. 41 - Componente $E_{z}$ referente ao choque entre feixes ópticos defasados de $180^{\circ}$ para um ângulo de propagação de $3^{\circ}$ em relação ao plano horizontal. 


\subsubsection{Passagem de um feixe condensado através de uma fenda.}

Nesta seção são mostrados os resultados para a incidência de um feixe óptico em uma fenda metálica. O metal usado é o ouro, que pode ser expresso em $\lambda=1,6 \mu m$ pelo índice de refração $n=0,448$ - j8,95. A Fig. 42 Ilustra o formato da fenda, a qual está situada em $z=0,5 \mathrm{~mm}$ e estende-se por $1 \mu \mathrm{m}$.
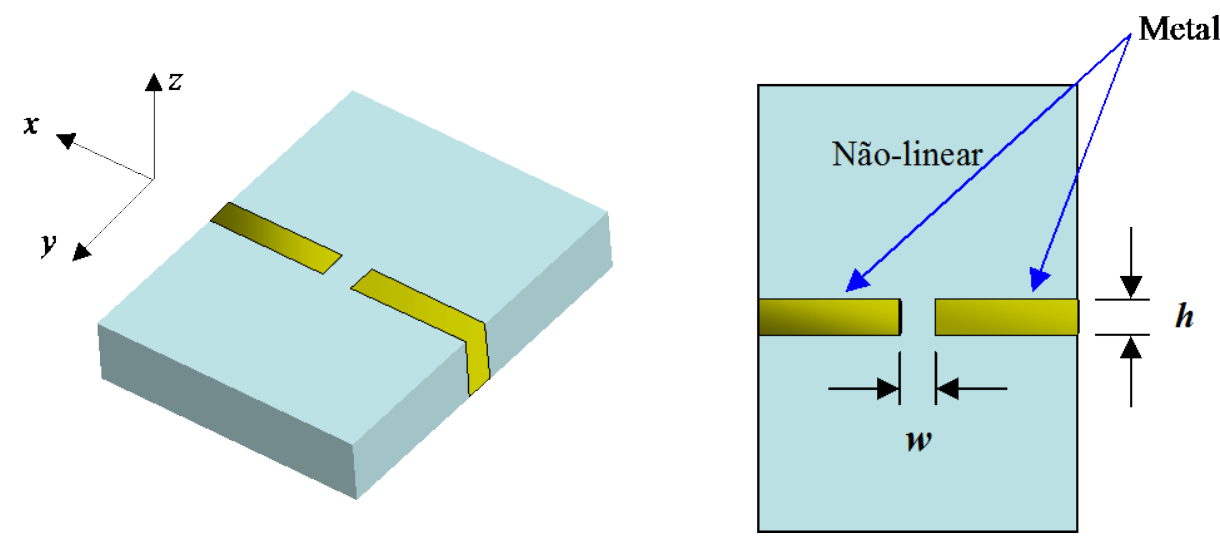

Fig. 42 - Ilustração da fenda metálica inserida no meio não-linear.

As figuras a seguir ilustram a incidência e passagem de um feixe supergaussiano através de fendas apresentando altura $h=30 \mu \mathrm{m}$ e aberturas $(w)$ de 1,0 $\mu \mathrm{m}$ (Fig. 43 a Fig. 45); 2,5 $\mu \mathrm{m}$ (Fig. 46 a Fig. 48) e 5,0 $\mu \mathrm{m}$ (Fig. 49 a Fig. 51). O resultado é que, além da difração observada, uma parcela da potência óptica incidente é mantida na forma de um feixe estabilizado com um diâmetro menor na saída. Identifica-se, portanto, duas situações: $\mathrm{Na}$ primeira tem-se campos difratados que, por apresentarem amplitudes muito baixas em relação à do feixe original, se propagam sem influência perceptível do meio não-linear, enquanto que na segunda, a "tensão superficial" mantém a consistência de parte da potência óptica incidente, evitando que a luz difrate por completo. A análise a seguir leva em conta a parcela de energia óptica que se manteve guiada.

Na Fig. 43, onde $w=1,0 \mu \mathrm{m}$, o feixe transmitido logo após a fenda $(\mathrm{z}=0,55)$ é contraído como se forças de Van der Waals estivessem atuando a fim de restaurar o seu equilíbrio, o qual é atingido quando o feixe adquire um formato circular devido à "tensão superficial". Portanto, apesar dos efeitos de difração (tendência de expansão horizontal do feixe) e do formato retangular da abertura da fenda, o feixe caracterizado numa região de alta intensidade retorna a um formato circular. As Figs. 
44 e 45 ilustram o comportamento das componentes de campo $E_{y}$ e $E_{z}$ ao longo da propagação.

Na Fig. 46, onde $w=2,5 \mu \mathrm{m}$, o perfil de luz tende claramente a se dividir em dois canais separados, o que é observado em $z=1,3 \mathrm{~mm}$. Aqui um feixe mais intenso, se comparado ao exemplo anterior, emerge após a fenda. Também neste caso as forças de Van de Waals impedem que o feixe se desintegre à medida que se propaga. Observe que neste caso o feixe apresenta a tendência de se expandir tanto horizontal (difração) quanto verticalmente, como pode ser observado pela distribuição de campo em $\mathrm{z}=0,55 \mathrm{~mm}$. No processo de expansão vertical há uma clara tendência na emissão de dois sólitons. Este dois sólitons são atraídos novamente e forçados a se colidirem. Por estarem em fase, a colisão (neste caso quase-elástica, por haver ruptura dos feixes) gera dois novos sólitons idênticos. Estes, por se comportam como dois guias de ondas idênticos e portanto satisfazem a condição de casamento de fase, coalescem novamente em um único fluxo óptico, conforme mostrado em $z=4000 \mu \mathrm{m}$. As Figs. 47 e 48 ilustram o comportamento das componentes de campo $E_{y}$ e $E_{z}$ ao longo da propagação.

Na Fig. 49, onde $w=5 \mu \mathrm{m}$, a formação de canais isolados não ocorre, apesar de ser esta a tendência. Isto deve-se ao fato da propagação do feixe ser pouco afetada pelo efeito de difração causada pela fenda. Assim, os centros de equilíbrios gerados pela contração do feixe não se afastam o suficiente para caracterizar a ruptura. As Figs. 50 e 51 ilustram o comportamento das componentes de campo $E_{y}$ e $E_{z}$ ao longo da propagação.

Os ruídos numéricos observados, principalmente na para a componente minoritária $E_{y}$, são provavelmente conseqüência dos campos difratados que superaram os limites paraxiais impostos no formalismo FD-BPM tridimensional. As TABELAS 14-16 listam as relações de amplitude entre as componentes minoritárias e a majoritária.

A Fig. 52 ilustra a evolução da intensidade do pulso ao longo da direção de propagação, onde observa-se quantitativamente a redução da potência guiada a partir da posição da fenda, para alguns valores de sua abertura $w$. A intensidade é calculada por meio de $I(z)=\iint|\bar{E}(x, y, z)|^{2} \partial x \partial y$. 
TABELA 14 - Relação entre as componentes minoritárias e a majoritária para a incidência de condensado óptico em fenda de 1,0 $\mu \mathrm{m}$ de abertura (v. Fig. 43 a Fig. 45)

\begin{tabular}{|c|c|c|c|}
\hline $\boldsymbol{z}(\boldsymbol{m m})$ & $\boldsymbol{E}_{\boldsymbol{y}} / \boldsymbol{E}_{\boldsymbol{x}}$ & $\boldsymbol{E}_{z} / \boldsymbol{E}_{\boldsymbol{x}}$ & $\boldsymbol{E}_{\boldsymbol{x}}(\boldsymbol{M V} / \boldsymbol{m})$ \\
\hline 0,0 & 0 & 0,02262299798913 & 184,0 \\
\hline 0,5 & 0,01877044613634 & 0,00080141316803 & 191,373137 \\
\hline 0,55 & 0,01402540608686 & 0,15433671230449 & 109,688911 \\
\hline 0,6 & 0,00387856086260 & 0,04365263228127 & 121,131394 \\
\hline 0,9 & 0,00108918739287 & 0,03028339180855 & 183,390516 \\
\hline 4,0 & 0,00051078836402 & 0,02487862735608 & 178,619492 \\
\hline
\end{tabular}

TABELA 15 - Relação entre as componentes minoritárias e a majoritária para a incidência de condensado óptico em fenda de 2,5 $\mu \mathrm{m}$ de abertura (v. Fig. 46 a Fig. 48)

\begin{tabular}{|c|c|c|c|}
\hline $\boldsymbol{z}(\boldsymbol{m m})$ & $\boldsymbol{E}_{\boldsymbol{y}} / \boldsymbol{E}_{\boldsymbol{x}}$ & $\boldsymbol{E}_{\boldsymbol{z}} / \boldsymbol{E}_{\boldsymbol{x}}$ & $\boldsymbol{E}_{\boldsymbol{x}}(\boldsymbol{M V} / \boldsymbol{m})$ \\
\hline 0,0 & 0 & 0,02247990043478 & 184,0 \\
\hline 0,5 & 0,00409511333556 & 0,02032075147081 & 184,984218 \\
\hline 0,55 & 0,00941682458513 & 0,07140164455926 & 136,095552 \\
\hline 0,9 & 0,00147933275776 & 0,02584277036598 & 184,188459 \\
\hline 1,3 & 0,00091081984025 & 0,03097268869065 & 184,603123 \\
\hline 4,0 & 0,00053694047410 & 0,02400039587312 & 180,360820 \\
\hline
\end{tabular}

TABELA 16 - Relação entre as componentes minoritárias e a majoritária para a incidência de condensado óptico em fenda de $5 \mu \mathrm{m}$ de abertura (v. Fig. 49 a Fig. 51)

\begin{tabular}{|c|c|c|c|}
\hline $\boldsymbol{z}(\boldsymbol{m m})$ & $\boldsymbol{E}_{\boldsymbol{y}} / \boldsymbol{E}_{\boldsymbol{x}}$ & $\boldsymbol{E}_{\boldsymbol{z}} / \boldsymbol{E}_{\boldsymbol{x}}$ & $\boldsymbol{E}_{\boldsymbol{x}}(\boldsymbol{M V} / \boldsymbol{m})$ \\
\hline 0,0 & 0 & 0,02247990043478 & 184,0 \\
\hline 0,5 & 0,00377491390461 & 0,05142873319905 & 184,944565 \\
\hline 0,55 & 0,00931101016822 & 0,04980202362710 & 164,209898 \\
\hline 0,9 & 0,00200596063589 & 0,02211249105506 & 189,426937 \\
\hline 1,1 & 0,00145783433343 & 0,02613907244912 & 171,543452 \\
\hline 4,0 & 0,00114995265589 & 0,02805335028210 & 182,940401 \\
\hline
\end{tabular}



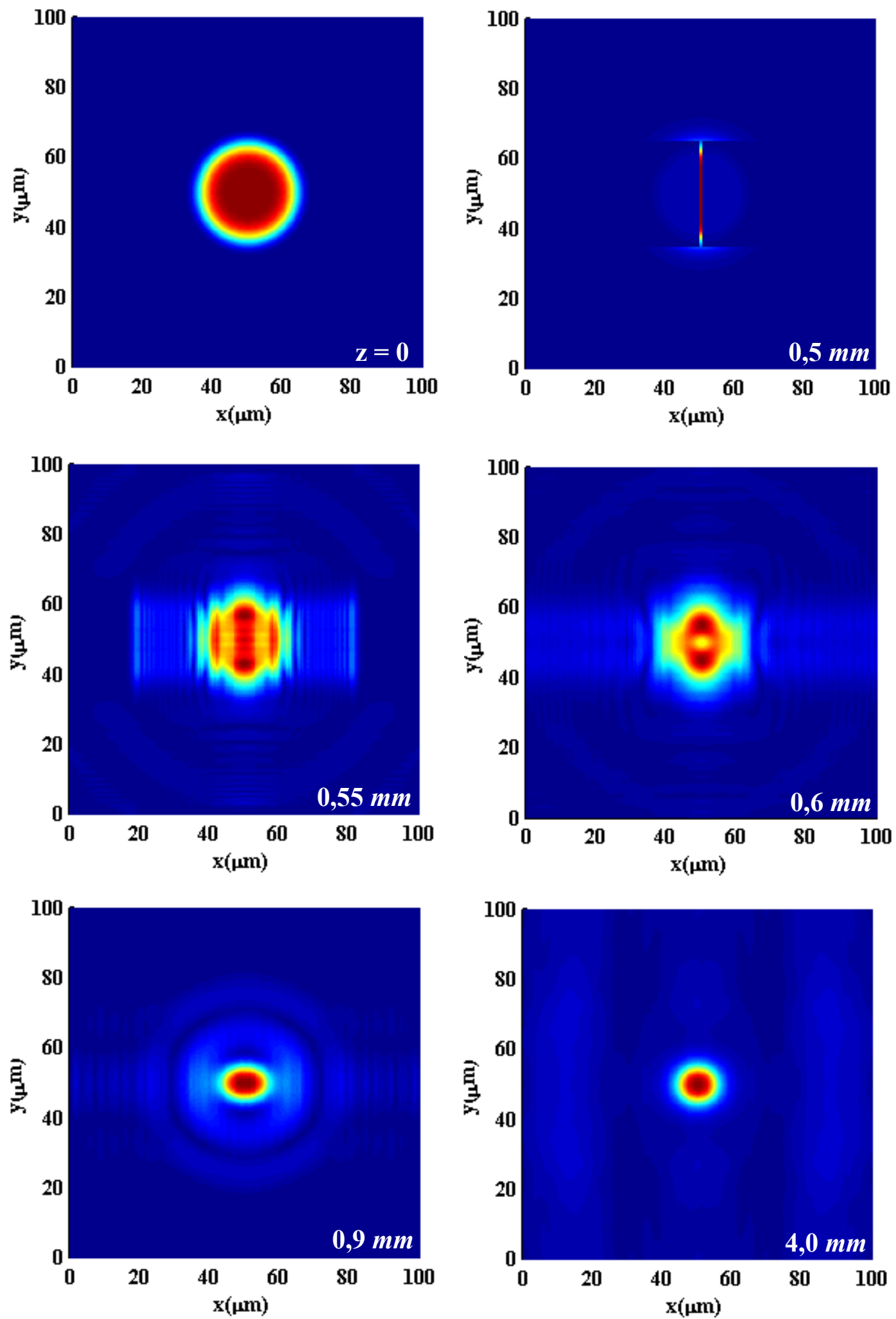

Fig. 43 - Componente dominante $E_{x}$ referente à incidência de um feixe óptico numa fenda metálica de $1,0 \mu \mathrm{m}$ de abertura. 

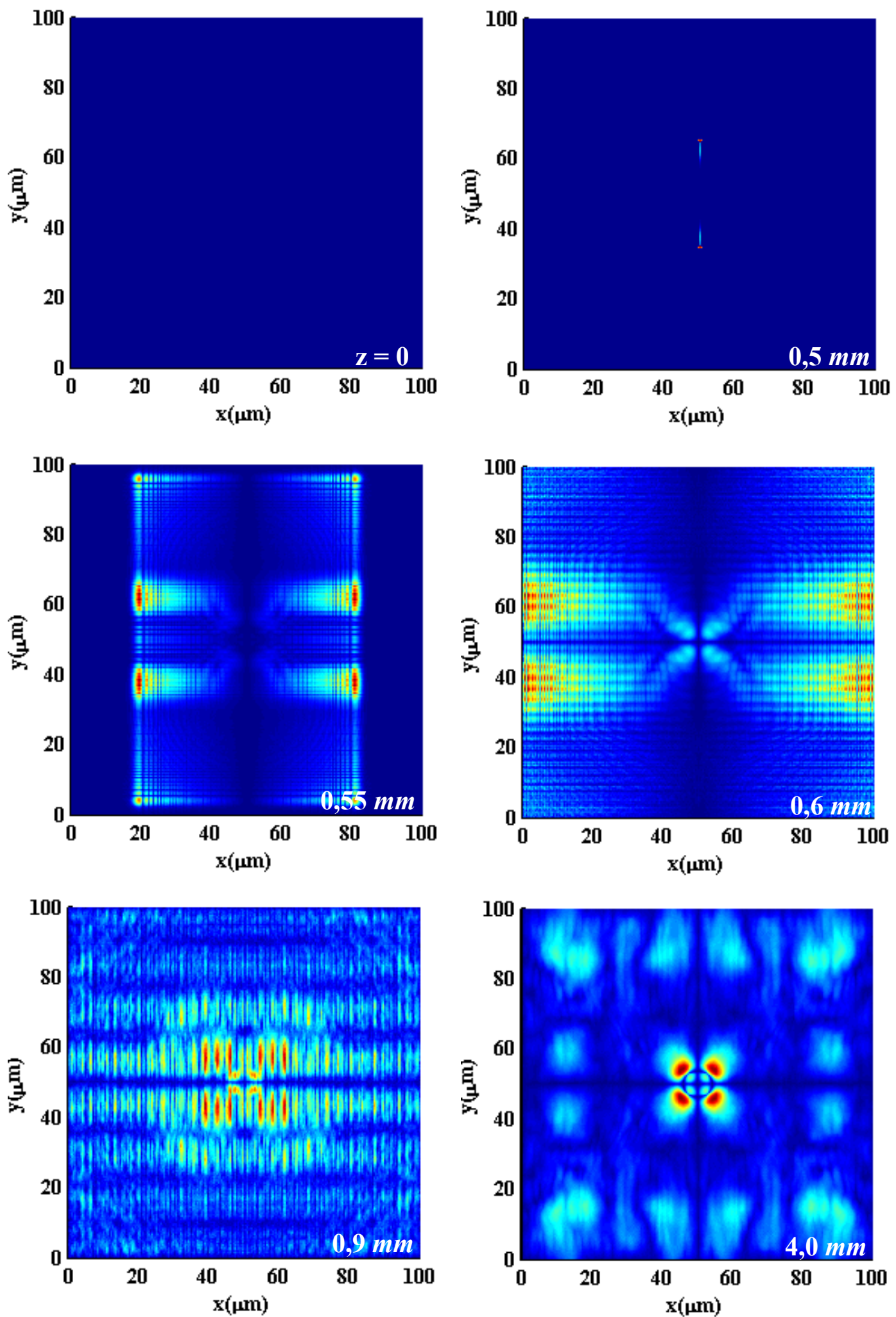

Fig. 44 - Componente minoritária $E_{y}$ referente à incidência de um feixe óptico numa fenda metálica de $1,0 \mu \mathrm{m}$ de abertura. 

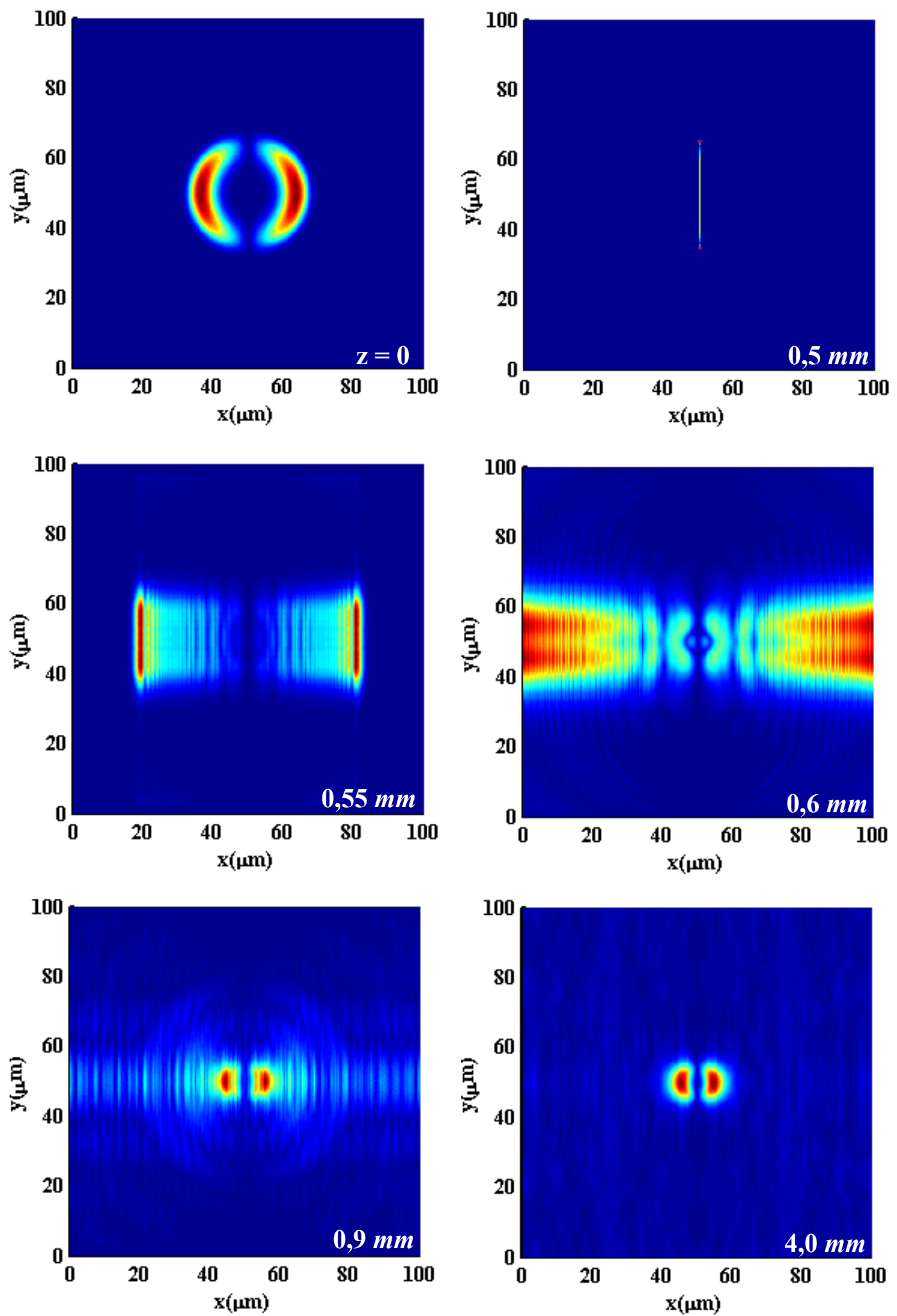

Fig. 45 - Componente minoritária $E_{z}$ referente à incidência de um feixe óptico numa fenda metálica de $1,0 \mu \mathrm{m}$ de abertura. 

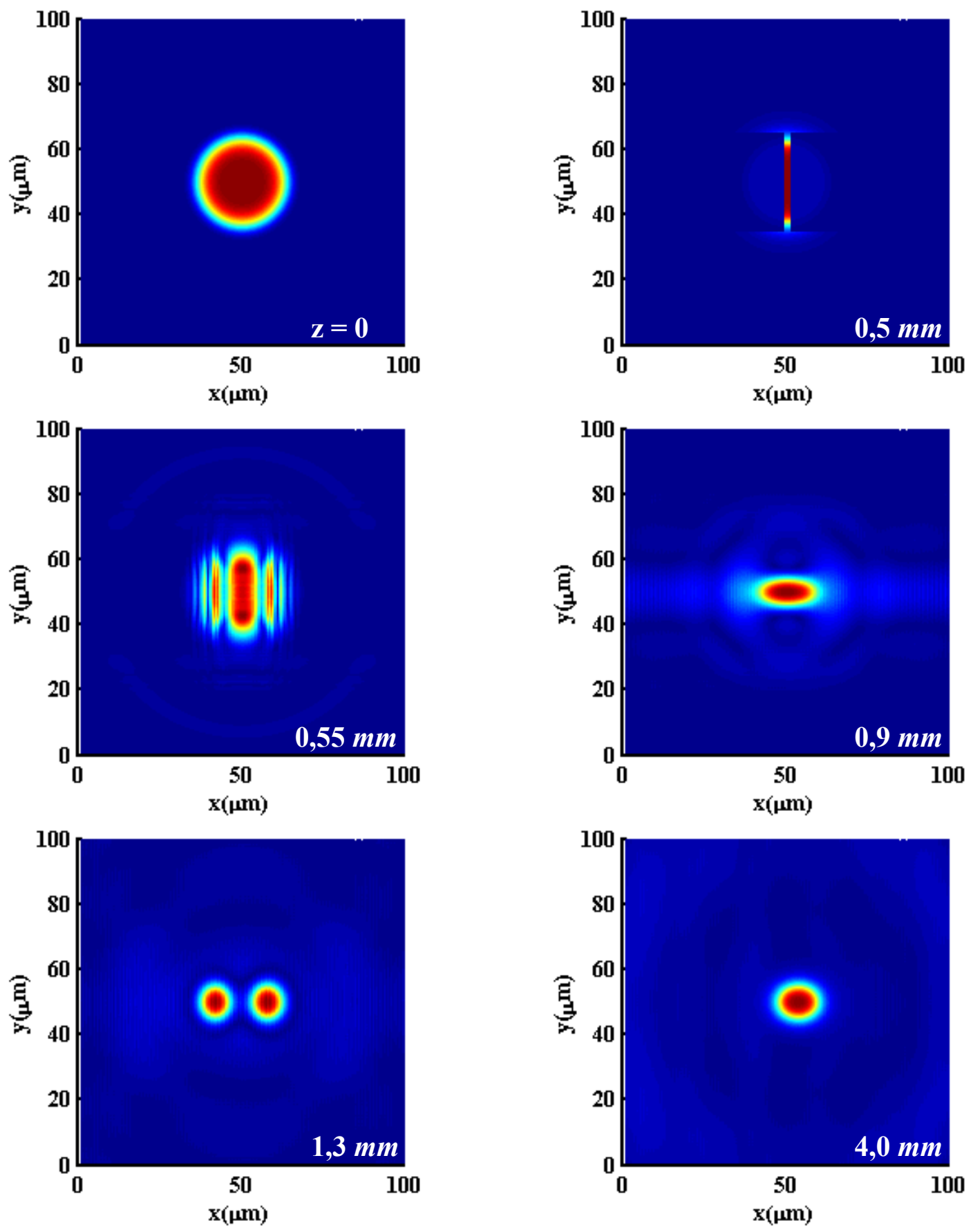

Fig. 46 - Componente dominante $E_{x}$ referente à incidência de um feixe óptico numa fenda metálica de $2,5 \mu \mathrm{m}$ de abertura. 

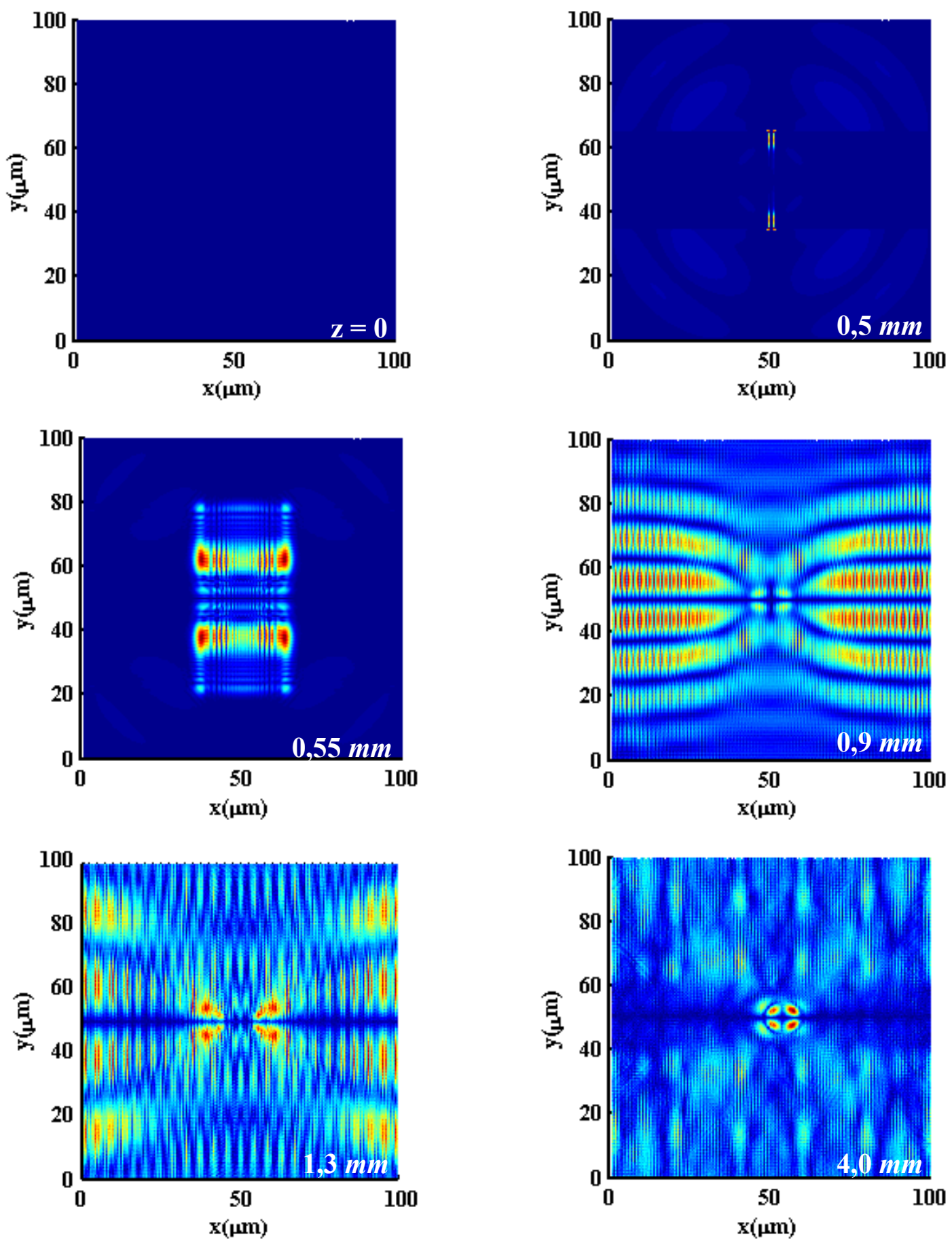

Fig. 47 - Componente minoritária $E_{y}$ referente à incidência de um feixe óptico numa fenda metálica de $2,5 \mu \mathrm{m}$ de abertura. 

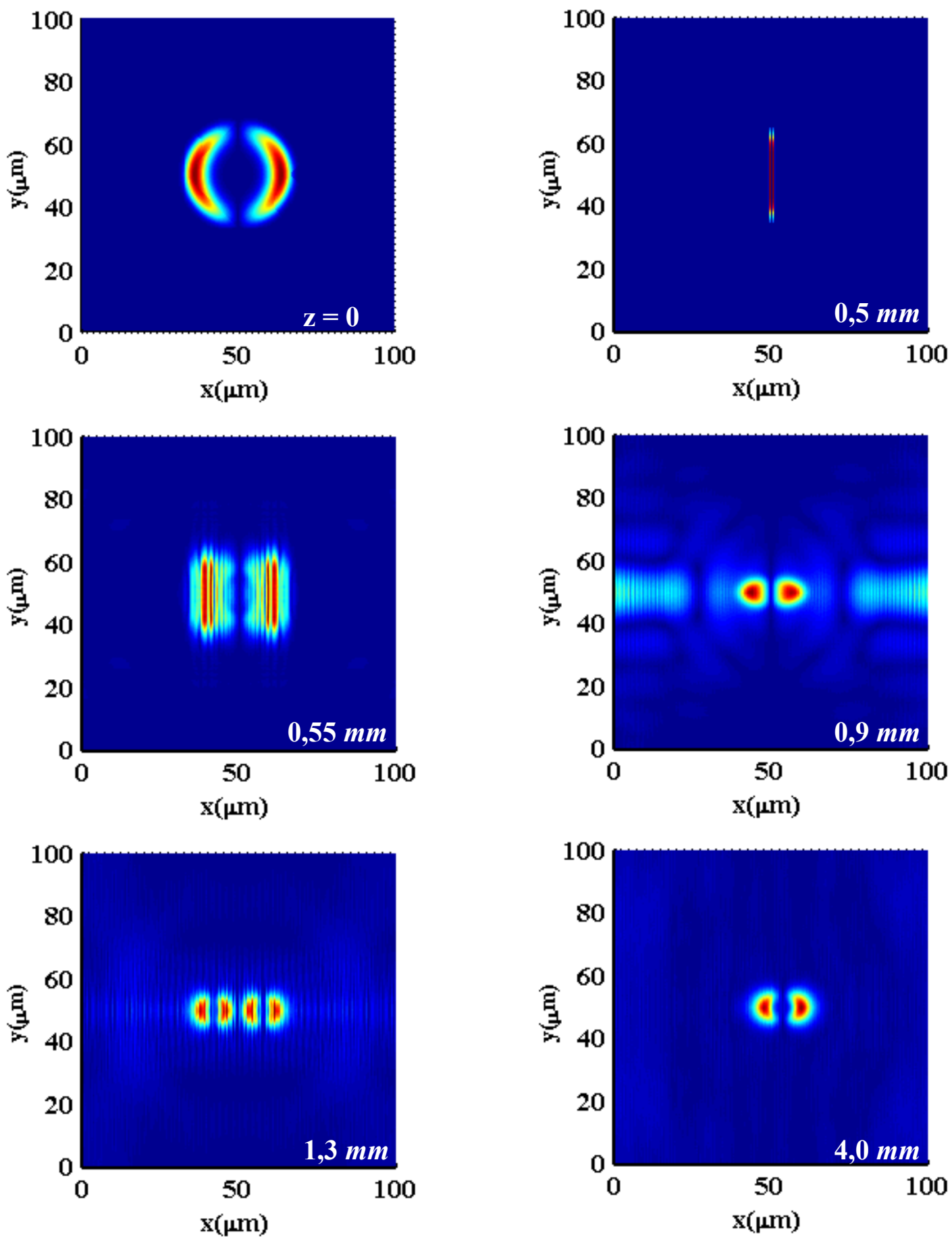

Fig. 48 - Componente minoritária $E_{z}$ referente à incidência de um feixe óptico numa fenda metálica de $2,5 \mu \mathrm{m}$ de abertura. 

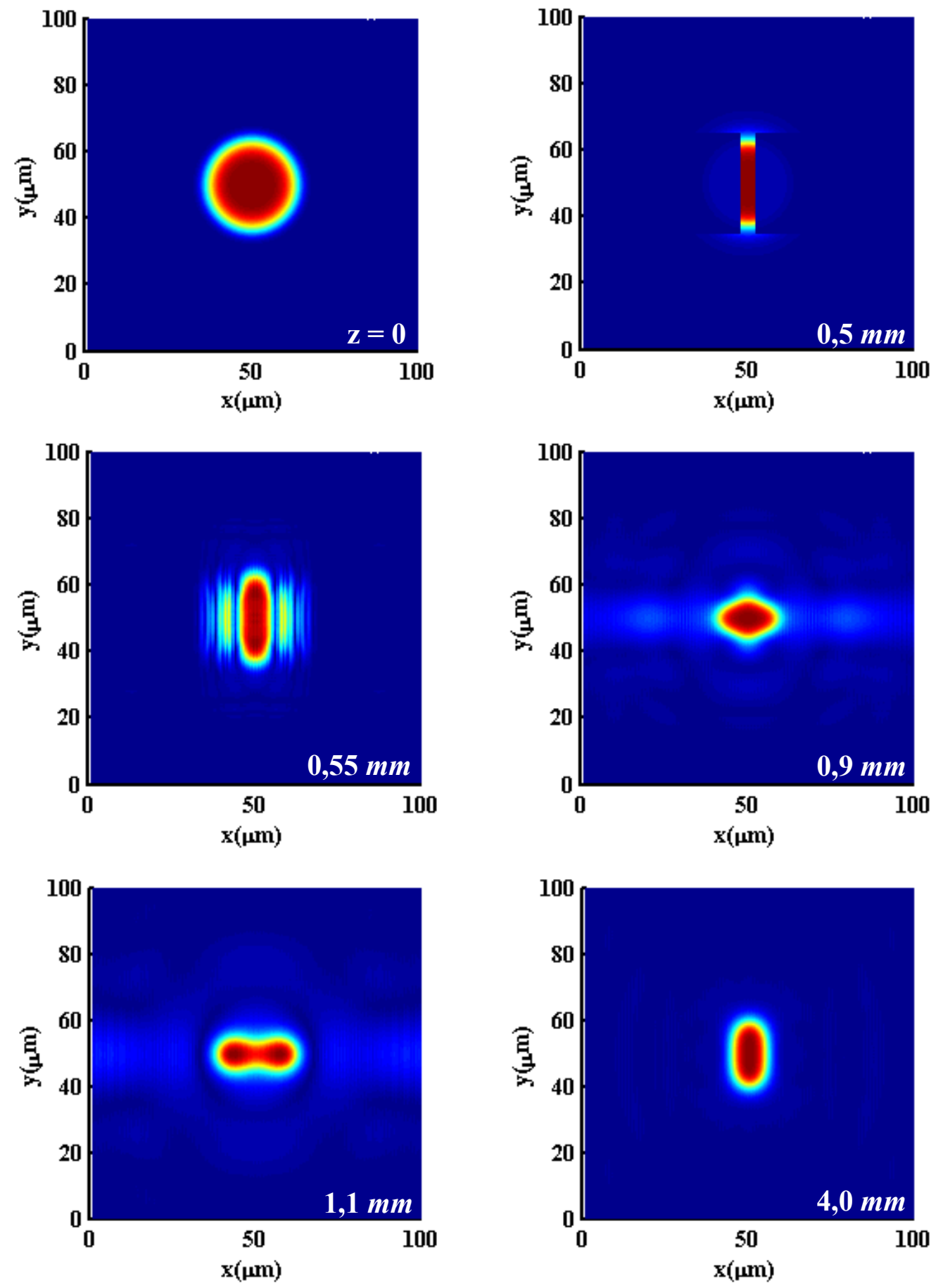

Fig. 49 - Componente dominante $E_{x}$ referente à incidência de um feixe óptico numa fenda metálica de $5,0 \mu \mathrm{m}$ de abertura. 

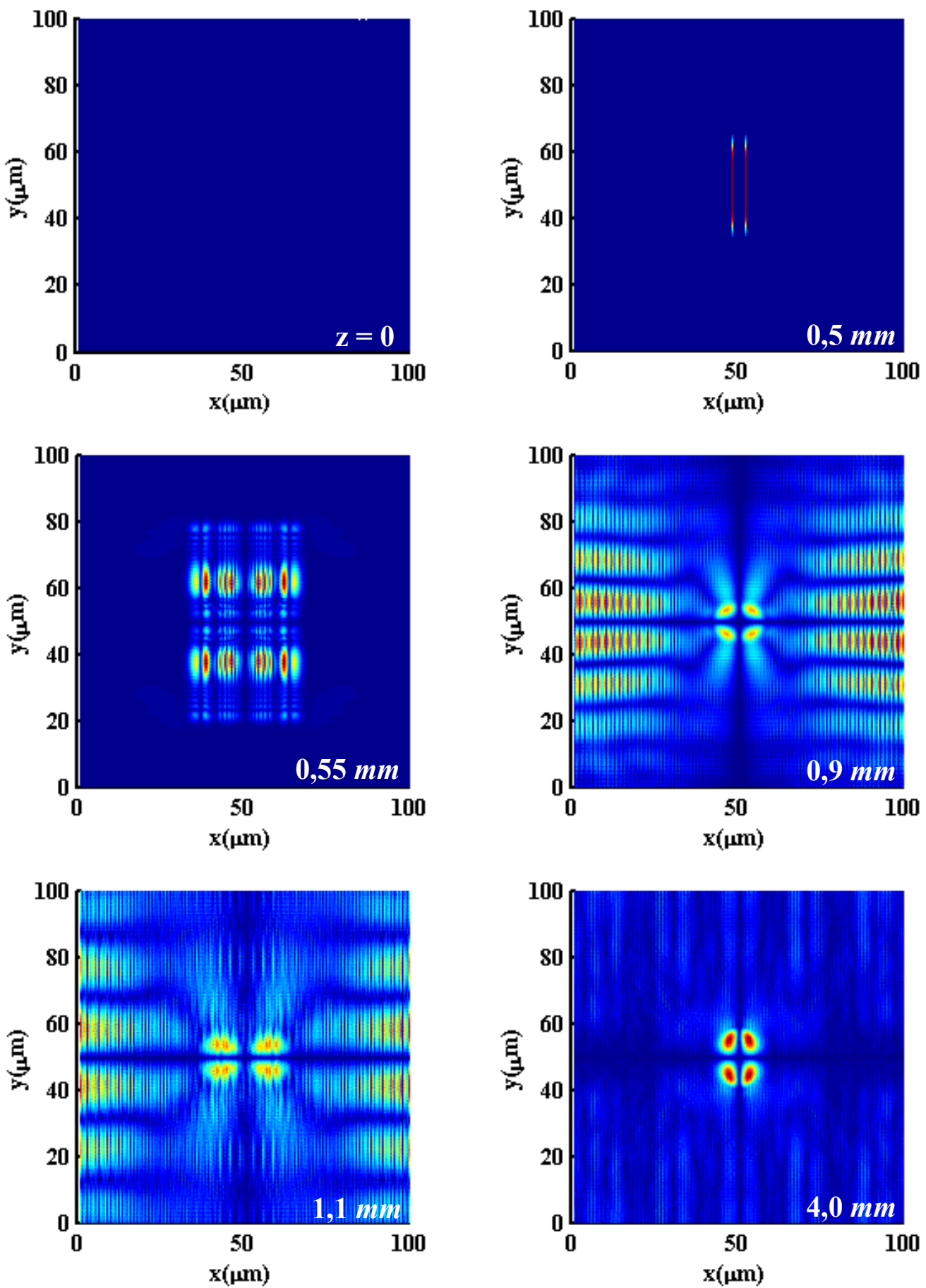

Fig. 50 - Componente minoritária $E_{y}$ referente à incidência de um feixe óptico numa fenda metálica de $5,0 \mu \mathrm{m}$ de abertura. 

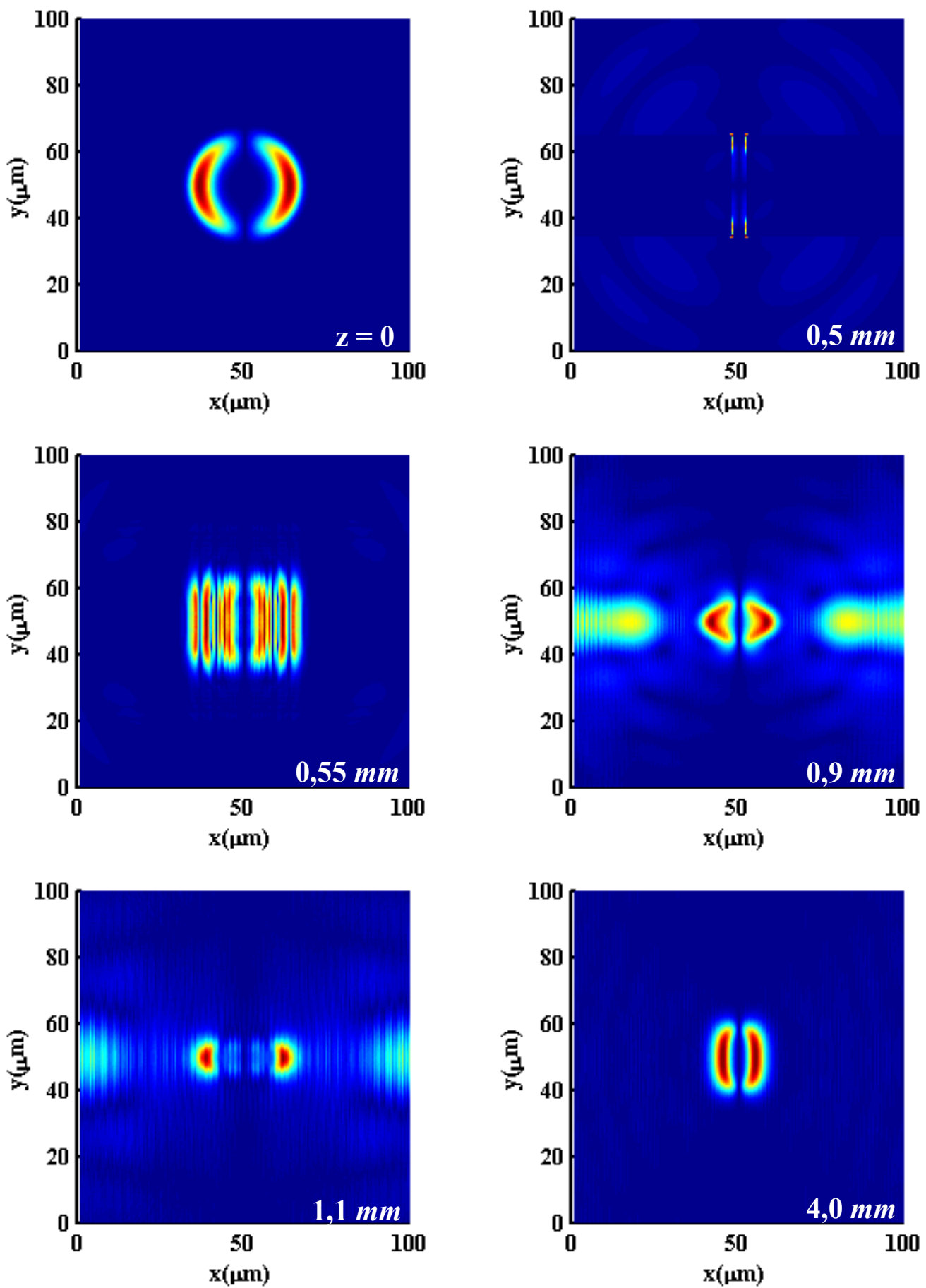

Fig. 51 - Componente minoritária $E_{z}$ referente à incidência de um feixe óptico numa fenda metálica de $5,0 \mu \mathrm{m}$ de abertura. 


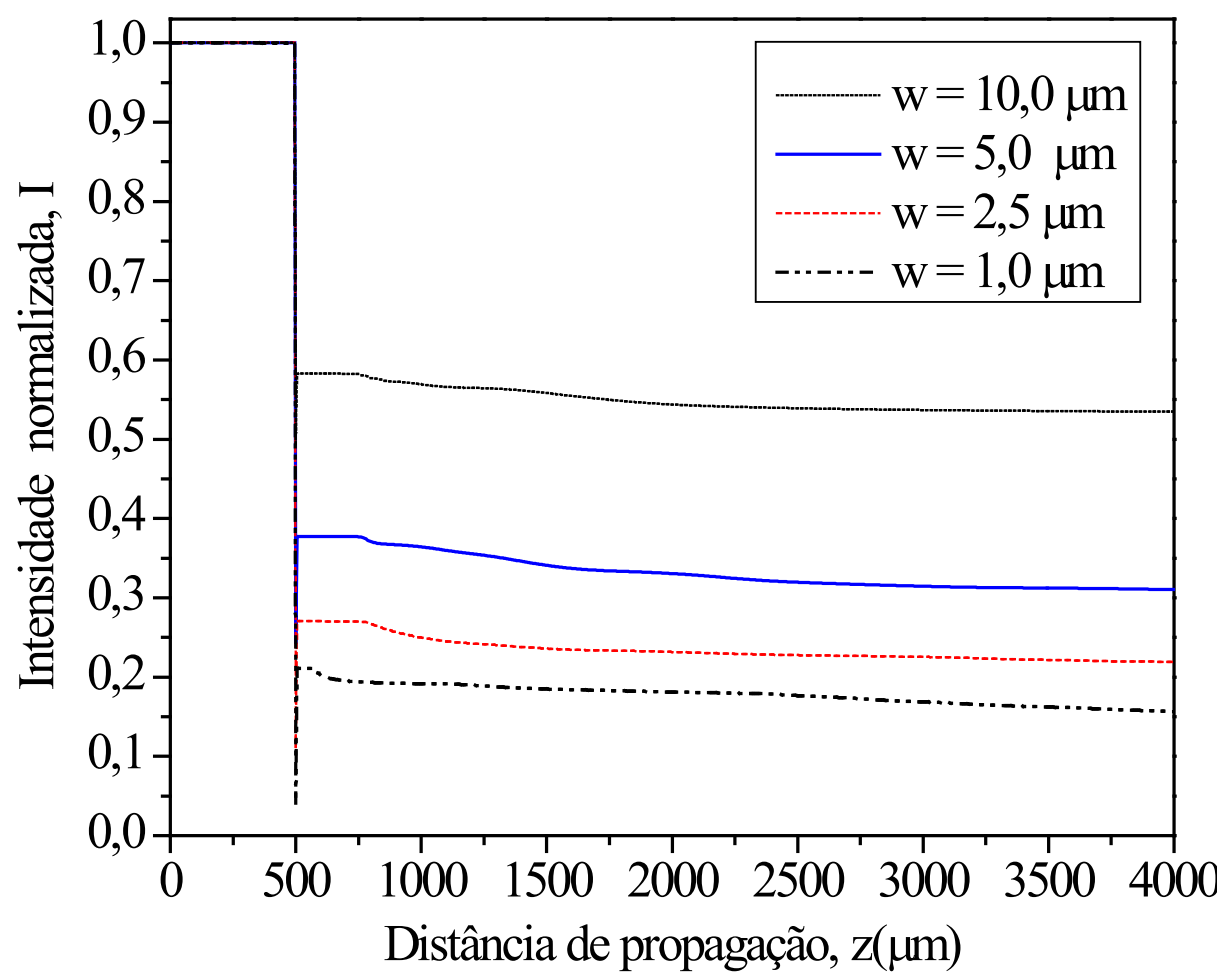

Fig. 52 - Evolução da intensidade de campo elétrico em função da distância de propagação. A fenda está situada em $\mathrm{z}=500 \mu \mathrm{m}$. 


\section{CONCLUSÕES}

Este trabalho demonstrou a aplicabilidade dos métodos FDTD e FD-BPM na simulação da propagação de ondas eletromagnéticas em estruturas contendo materiais com propriedades ópticas distintas. A princípio, foram realizadas simulações de modos TM em guias planares contendo camadas linear, não-linear e não-recíproca, das quais foram obtidos resultados inéditos na literatura por meio do método FDTD.

A possibilidade de modelar problemas desta natureza utilizando o método FDTD já era esperada, devido à sua versatilidade em diversas aplicações de eletromagnéticas. Por lidar diretamente com as equações de Maxwell, o FDTD é inerentemente vetorial e relativamente simples de ser formulado. O domínio do tempo possibilita a investigação de excitações pulsadas ou a análise de transientes. Outra característica vantajosa consiste está no fato da direção de propagação não ser predeterminada. Isso possibilita a plena análise de dispositivos baseados em guias curvos ou inclinados, além da radiação eletromagnética em espaço livre. O único fator que pode dificultar sua realização é que este método é numericamente intensivo e normalmente requer recursos computacionais de vanguarda, tais como supercomputadores, ou técnicas especiais de programação para clusters de processamento paralelo.

Adicionalmente, um formalismo inédito baseado no método numérico FD-BPM, visando a simulação da propagação de ondas eletromagnéticas em estruturas que podem conter simultaneamente materiais isotrópicos, anisotrópicos e não-lineares foi também proposto e implementado. A princípio, para validar a técnica, a análise das propriedades não-lineares e não-recíprocas de um guia planar foi realizada e comparada com os resultados obtidos por intermédio do método FDTD, apresentando uma boa concordância com este último. Um projeto de um acoplador 
direcional não-linear e não-recíproco que acumula as funções de chave óptica e de isolador óptico também foi proposto.

O FD-BPM possibilita o uso de passos longitudinais maiores em relação ao método FDTD, devido à aplicação da aproximação de envoltória lenta (SVEA). Com isso, a precisão do método torna-se menos sensível ao passo longitudinal, para uma determinada (e restrita) faixa de constantes de propagação modais, o que depende do valor de índice efetivo de referência adotado. Dessa forma, estruturas mais longas podem ser simuladas com precisão similar ao FDTD, mas com rapidez muito maior. Por ser um método no domínio da freqüência, o domínio espacial inteiro não necessita ser atualizado a cada passo de tempo, como é o caso do método FDTD. Um método no domínio da freqüência se torna mais conveniente quando uma resposta em regime estacionário é desejada. Contudo, o que faz o FD-BPM mais eficiente em relação ao FDTD, também o torna menos geral. Um exemplo disso é em relação à direção de propagação, que não pode apresentar uma grande variação em relação ao eixo longitudinal. Adicionalmente, reflexões (casos extremos) não poderiam ser observadas. Métodos baseados no domínio da freqüência não podem simular sinais pulsados ou de banda larga. Mesmo apresentando estas limitações, o método FDBPM desenvolvido neste trabalho vem se constituindo uma excelente ferramenta de projeto bem como um ótimo benchmark para o método FDTD originalmente proposto.

Finalmente, mesmo com as restrições observadas acima, o FD-BPM requer uma quantidade de processamento bem menor que o método FDTD na simulação de um mesmo problema. As possibilidades de aplicação do método FDTD em problemas eletromagnéticos são praticamente infinitas e a barreira geralmente é a imensa quantidade de processamento necessária em relação aos sistemas computacionais disponíveis. O FD-BPM aplicado a este projeto foi proposto como uma alternativa de menor esforço computacional que, mesmo não sendo tão geral quanto o método FDTD, ainda é capaz de resolver vários problemas de óptica guiada.

Em virtude desta maior eficiência numérica, esforços foram feitos na implementação de uma versão tridimensional para o FD-BPM. Com o objetivo de caracterizar precisamente a fenomenologia de efeitos não-lineares, o método FDBPM vetorial para meios anisotrópicos descrito nesta tese também é estendido para a 
consideração de meios não-lineares pela inclusão de procedimento iterativo para a convergência da permissividade dielétrica em função do campo elétrico. O fato desta permissividade ser tensorial facilita a consideração, caso seja necessário, de nãolinearidades anisotrópicas que são observadas para certos mecanismos de nãolinearidade. Dessa forma, uma ampla lista de efeitos não-lineares pode ser analisada, inclusive em conjunto com materiais anisotrópicos lineares. O método proposto combina pela primeira vez as técnicas implícita de direções alternantes (ADI) e leapfrog para a simulação eficiente da propagação óptica em estruturas tridimensionais. O êxito do formalismo foi comprovado na simulação de um guia $r i b$ magnetoóptico, o qual atua como um isolador óptico baseado em rotação de Faraday e também na simulação de feixes ópticos "condensados" que propagam num meio não-linear do tipo Kerr com saturação. Dessa forma, a simulação de problemas tridimensionais, como originalmente previsto neste projeto, pôde ser investigada.

Como proposta de trabalhos futuros, sugere-se a otimização do dispositivo híbrido chave-isolador óptico através de algoritmos genéticos. Outra sugestão é a investigação de novos fenômenos com o método FD-BPM 3D vetorial elaborado, como, por exemplo, a propagação de vórtices de luz. Um vórtice de luz consiste em um feixe apresentando singularidade (indeterminação) de fase ao longo de uma linha paralela ao seu eixo, o que pode ser visto como uma interferência destrutiva. Ao redor do núcleo escuro do vórtice óptico, a energia transportada pela luz flui como uma escadaria em espiral, seguindo um sentido horário ou anti-horário. Pesquisas vêm sendo realizadas visando a aplicação de vórtices de luz como "motores" de dispositivos MEMS (Microelectromechanical System) ${ }^{(1)}$. Outra alternativa de trabalho interessante seria a implementação do método FDTD tridimensional baseado na célula de "dois nós" para a análise de light bullets em meios do tipo Kerr com saturação, o que provavelmente exigirá a aplicação de técnicas de processamento paralelo (e, obviamente, a disponibilidade de um cluster de processamento distribuído).

(1) http://www.nanoelectronicsplanet.com/nanochannels/ research/article/0,4028,10497_2177301,00.html 


\section{REFERÊNCIAS}

[1] R. M. Joseph e A. Taflove, "FDTD Maxwell's equations models for nonlinear electrodynamics and Optics," IEEE Trans. Antennas Propagat., vol. 45, pp. 364-374, Março de 1997.

[2] A. Taflove, Computational Electrodynamics: The Finite-Difference TimeDomain Method, Artech House, Boston, MA, 1998.

[3] A. Taflove, "Review of the formulation and applications of the finite-difference time-domain method for numerical modeling of electromagnetic wave interactions with arbitrary structures," Wave Motion, vol. 10, no. 6, pp. 547-582, 1988.

[4] J. B. Schneider e S. Hudson, "The finite-difference time-domain method applied to anisotropic material," IEEE Trans. Antennas Propagat., vol. 41, pp. 994-999, Julho de 1993

[5] D. E. Merewether e W. A. Radasky, "Nonlinear electromagnetic fields within a cylindrical cavity excited by ionizing radiation," IEEE Trans. Nucl. Sci., vol. 21, no. 2, pp. 998-1005, 1974.

[6] R. Luebbers, K. Kumagai, S. Adachi, e T. Uno, "FDTD calculation of transient pulse propagation through a nonlinear magnetic sheet," IEEE Trans. Electromagn. Compat., vol. 35, pp. 90-94, Fevereiro de 1993.

[7] H. A. Jamid e S. J. Al-Bader, "Finite-difference time-domain approach to nonlinear guided-waves," Electron. Lett., vol. 29, pp. 83-84, Janeiro de 1993.

[8] R. W. Ziolkowski e J. B. Judkins, "Applications of the nonlinear finite difference time domain (NL-FDTD) method to pulse propagation in nonlinear media: Selffocusing and linear-nonlinear interfaces," Radio Sci., vol. 28, pp. 901-911, Setembro-Outubro de 1993.

[9] R. W. Ziolkowski e J. B. Judkins, "Full-wave vector Maxwell equation modeling of the self-focusing of ultrashort optical pulses in a nonlinear Kerr medium 
exhibiting a finite response time," J. Opt. Soc. Am., B Opt. Phys., vol. 10, pp. 186-198, Fevereiro de 1993.

[10] R. W. Ziolkowski e J. B. Judkins, "Nonlinear finite-difference time-domain modeling of linear and nonlinear corrugated waveguides," J. Opt. Soc. Am., B Opt. Phys., vol. 11, pp. 1565-1575, Setembro de 1994.

[11] R. W. Ziolkowski, "The incorporation of microscopic material models into the FDTD approach for ultrafast optical pulse simulations," IEEE Trans. Antennas Propagat., vol. 45, pp. 375-391, Março de 1997.

[12] A. Reineix, M. Ariaudo, B. Jecko, e A. Barthelemy, "Adaptation of the FDTD method to the study of femtosecond electromagnetic soliton," Microwave Opt. Technol. Lett., vol. 7, no. 11, pp. 507-511, 1994.

[13] T. Shiozawa e T. Yoshitake, "Efficiency enhancement in a Cherenkov laser loaded with a Kerr-like medium," IEEE J. Quantum Electron., vol. 31, pp. 539545, Março de 1995.

[14] D. M. Sullivan, "Nonlinear FDTD formulations using Z transforms," IEEE Trans. Microwave Theory Tech., vol. 43, pp. 676-682, Março de 1995.

[15] D. M. Sullivan, "Z-transform theory and the FDTD method," IEEE Trans. Antennas Propagat., vol. 44, pp. 28-34, Janeiro de 1996.

[16] P. Tran, "Optical switching with a nonlinear photonic crystal: A numerical study," Optics Lett., vol. 21, pp. 1138-1140, Agosto de 1996.

[17] K. L. Shlager e J. B. Schneider, "A Selective Survey of the Finite-Difference Time-Domain Literature", IEEE Antennas and Propagation Magazine, vol. 37, no. 4, pp. 39-56, 1995.

[18] G. Mur, "Absorbing boundary conditions for the finite-difference approximation of the time-domain electromagnetic-field equations," IEEE Transactions on Electromagnetic Compatibility, vol. EMC-23, pp. 377-382, Novembro de 1981.

[19] R. L. Higdon, “Absorbing boundary conditions for difference approximations to the multi-dimensional wave equations," Math. Comput., vol. 47, pp. 437-459, Outubro de 1986.

[20] R. L. Higdon, "Numerical absorbing boundary conditions for the wave equation," Math. Comput., vol. 49, pp. 65-90, Julho de 1987. 
[21] J. P. Berenger, "A perfectly matched layer for the absorption of electromagnetic waves," Journal of Computational Physics, vol. 114, no. 1, pp. 185-200, 1994.

[22] W. C. Chew e W. H. Weedon, “A 3D perfectly matched medium from modified Maxwell's equations with stretched coordinates," Microwave and Optical Technology Letters, vol. 7, pp. 599-604, Setembro de 1994.

[23] D. S. Katz, E. T. Thiele, e A. Taflove, "Validation and extension to three dimensions of the Berenger PML absorbing boundary condition for FD-TD meshes," IEEE Microwave and Guided Wave Letters, vol. 4, pp. 268-270, Agosto de 1994.

[24] R. Mittra e U. Pekel, "A new look at the perfectly matched layer (PML) concept for the reflectionless absorption of electromagnetic waves," IEEE Microwave and Guided Wave Letters, vol. 5, no. 3, pp. 84-86, 1995.

[25] D. M. Sullivan, "A simplified PML for use with the FDTD method," IEEE Microwave and Guided Wave Letters, vol. 6, no. 2, pp. 97-99, Fevereiro de 1996.

[26] A. P. Zhao, J. Juntunen and A. V. Raisanen, “Generalized-Material-Independent PML absorbers used for the FDTD simulation of electromagnetic waves in arbitrary anisotropic dielectric and magnetic media", IEEE Microwave and Guided Wave Letters, vol. 8, no. 2, págs. 52-54, Fevereiro de 1998.

[27] M. D. Feit and J. A. Fleck, "Light propagation in graded-index optical Fibers", Appl. Opt., Vol. 17, pp. 3990-3997, 1978.

[28] G. R. Hadley, "Wide-angle beam propagation using Padé approximant operators", Opt. Lett., Vol. 17, pp. 1426-1428, 1992.

[29] C. Rappaport, "Perfectly matched absorbing boundary conditions based on anisotropic lossy mapping of space," IEEE Microwave and Guided Wave Lett., vol.5, pp. 90-92, Março de 1995.

[30] D. Jiménez, C. Ramírez, F. Pérez-Murano and A. Guzmán, “Implementation of Bérenger layers as boundary conditions for the beam propagation method: applications to integrated waveguides," Optics Communications, 159, pp. 43-48, Janeiro de 1999. 
[31] F. L. Teixeira and W. C. Chew, "A general aproach to extend Berenger's absorbing boundary condition to anisotropic and dispersive media," IEEE Trans. Antennas Propagat., vol.46, No. 9, pp. 1386-1387, Setembro de 1998.

[32] G. Hadley, "Transparent boundary condition for beam propagation”, Optics Letters, vol. 16, n. 9, pp. 624-626, Maio de 1991.

[33] L. Thylen and D. Yevick, "Beam Propagation method in anisotropic media", Appl. Opt., vol. 21, pp.2751-2754, Agosto de 1982.

[34] Y. Tsuji, M. Koshiba and N. Takimoto, "Finite-element Beam propagation method for anisotropic optical waveguides", J. Lightwave Technol., vol.17 no.4, pp.723-728, Abril de 1999 .

[35] E. A. C. Gonçalves, “Análise de dispositivos com materiais magnetoópticos para aplicações em sistemas de comunicações ópticas", Dissertação de Mestrado, EESC/USP, Setembro de 2001, São Carlos, SP.

[36] H. E. Hernández-Figueroa, "A new finite element scheme for optical temporal soliton analysis", International Conference on Computation in Electromagnetics, pp.167-169, Novembro de 1995.

[37] H. Yokota, M. Hira, and S. Kurazono, "Iterative Finite-Difference BeamPropagation Method analysis of nonlinear-optical waveguide excitation problem", Electronics and Communications in Japan Part II-Electronics, vol.78, pp. 73-80, Janeiro de 1995.

[38] T. Yasui and M. Koshiba, "A wide-angle finite-element beam propagation method with perfectly matched layers for nonlinear optical waveguides", $J$. Lightwave Technol., Vol.17, no. 10, pp. 1909-1915, Outubro de 1999.

[39] R. S. Flamino, "Método da propagação de feixe de ângulo largo para análise de guias de ondas ópticos não-lineares", Dissertação de Mestrado, EESC/USP, Setembro de 2001, São Carlos, SP.

[40] K. Gnideck, "TM waves in magnetooptic-nonlinear waveguide", Optica Applicata, vol. XXIV, n. 4, pp. 281-286, 1994.

[41] W. P. Huang and C. L. Xu, "Simulation of three-dimensional optical waveguides by a full-vector beam propagation,” IEEE J. Quantum Electron., vol. 29, pp. 2639-2649, 1993. 
[42] C. L. Xu, W. Huang, J. Chrostowski and S. Chaudhuri, "A full vectorial beampropagation method for anisotropic waveguides," J. Lightwave Technol., vol. 12, no. 11, pp. 1926-1931, Novembro de 1994.

[43] T. Yasui, M. Koshiba, A. Niiyama, and Y. Tsuji, "Finite element beam propagation method for nonlinear optical waveguides," Electronicas and Communications in Japan, Part 2, vol. 82, no. 4, pp. 47-53, 1999.

[44] S. Selleri, L. Vincetti, and M. Zoboli, "Full-vector finite-element beam propagation method for anisotropic optical device analysis," IEEE J. Quantum Electron., vol. 36, pp. 1392-1401, 2000.

[45] J. Yamauchi, T. Ando, and H. Nakano, "Beam-propagation analysis of optical fibers by alternating direction implicit method," Electron. Lett., vol. 27, pp. 1663-1665, 1991.

[46] P. L. Liu and B. J. Li, "Semivectorial beam-propagation method for analyzing polarized modes of rib waveguides," IEEE J. Quantum Electron., vol. 28, pp. 778-782, 1992.

[47] Y. Hsueh, M. Yang, and H. Chang, “Three-Dimensional noniterative fullvectorial beam propagation method based on the alternating direction implicit method.," J. Lightwave Technol., vol 17, pp. 2389-2397, Novembro de 1999.

[48] J. Yamauchi, G. Takahashi and H. Nakano, "Full Vectorial Beam-Propagation Method Based on the McKee-Mitchell Scheme with Improved Finite-Difference Formulas," J. Lightwave Technol., vol. 16, no. 12, pp. 2458 -2464, Dezembro 1998

[49] K. S. Yee, "Numerical solution of initial boundary value problems involving Maxwell's equations in isotropic media," IEEE Trans. on Antennas Propagat., vol. 14, pp. 302-307, Maio de 1966.

[50] Z. Bi, K. Wu, C. Wu, e J. Litva, "A new finite-difference time-domain algorithm for solving Maxwell's equations," IEEE Microwave Guided Wave Lett., vol. 1, no. 12, pp. 382-384, Dezembro de 1991.

[51] S. G. Garcia, T. M. Hung-Bao, R. G. Martín, e B. G. Olmedo, "On the application of finite methods in time domain to anisotropic dielectric waveguides," IEEE Trans. Microwave Theory Tech., vol. 44, no. 12, pp. 21952206, Dezembro de 1996. 
[52] A. Taflove and M. E. Brodwin, "Numerical Solution of Steady-State Electromagnetic Scattering Problems Using the Time-Dependent Maxwell's Equations," IEEE Trans. Microwave Theory Tech.,vol.23, no.8, pp.623 -630, Agosto de 1975.

[53] C. M. Furse, O. P. Ganghi, "Why the DFT is Faster than the FFT for FDTD Time-to-Frequency Domain Conversions," IEEE Microwave Guided Wave Lett., vol. 5, no. 10, pp. 326-328, Outubro de 1995.

[54] A. Taflove, Computational Electrodynamics - The Finite-Difference TimeDomain Method, Artech House, 1995.

[55] K. Ogusu, "TM waves guided by nonlinear planar waveguides", IEEE Trans. Microwave Theory Tech., vol.37, pp. 941-946, Junho de 1989.

[56] IMSL Math/Library Users Manual (IMSL Inc., 2500 CityWest Boulevard, Houston TX 77042).

[57] R. Wolfe, R. Lieberman, V. Fratello, R. Scotti, and N. Kopylov, "Etch-tuned ridged waveguide magneto-optic isolator," Appl. Phys. Lett., vol. 56, pp. 426428, Janeiro de 1990.

[58] R. McLeod, K. Wagner, and S. Blair, “(3+1)-dimensional optical soliton dragging logic," Phys. Rev. A, vol. 52, no. 4, pp. 3254-3283, Outubro de 1995.

[59] H. Michinel, J. Campo-Táboas, R. García-Fernandez, J. R. Salgueiro, and M. L. Quiroga-Teixeiro, "Liquid light condensates," Phys. Rev. E, vol. 65, pp. 066604(1-4), Junho de 2002.

[60] F. Smektala, C. Quemard, V. Couderc, and A. Barthélémy, "Non-linear optical properties of chalcogenide glasses measured by Z-scan," J. Non-Cryst. Solids, vol. 274, pp. 232-237, 2000.

[61] Snyder A.W. and Sheppard A.P., "Collisions, Steering, and Guidance with Spatial Solitons”, Opt. Lett., vol. 18, no. 7, pp. 482-484, April 11993.

[62] Wieslaw Królikowskiy, Cornelia Denzz, Andreas Stepkenz, Mark Saffmanx and Barry Luther-Daviesy, "Interaction of Spatial Photorefractive Solitons", Quantum Semiclass. Opt., 10 (1998), pp. 823-837.

[63] Steffen Kjær Johansen, Ole Bang, and Mads Peter Sørensen, "Escape angles in bulk $\chi^{(2)}$ soliton interactions", Physical Review E, vol. 65, 026601, 1-4, 2002. 
[64] Benkui Tan and John P. Boyd, "Coupled-mode envelope solitary waves in a pair of cubic Schrödinger equations with cross modulation: Analytical solution and collisions with application to Rossby waves", Chaos, Solitons, \& Fractals, vol. 11, no. 7, pp. 1113-1129, 1 June 2000.

[65] William C.K. Mak, Boris A. Malomed, and Pak L. Chu, "Formation of a Standing-Light Pulse through Collision of Gap Solitons", Physical Review E (Stat., Nonlin., and Soft Matter Phys.), vol. 68, Part 2, 026609, Aug 22, 2003. 


\section{APÊNDICE A - Algoritmo do Método FDTD}

Início do Algoritmo

Definir discretização da malha.

Obter distribuição transversal de campo para células em $z=0$ e componentes $H_{y 0}(x$, $\delta z / 2), D_{x 0}(x, 0), D_{z 0}(x, \delta z / 2)$ (campo de excitação).

Definir função temporal de excitação $f_{T}(\mathrm{t})$.

Inicializar componentes de campo (com valor nulo ou de excitação, dependendo da posição).

Início do laço de tempo $(t)$

Início dos laços espaciais $(z, x)$

Atualizar $H_{y}$ [Eq. (2.36) ou Eqs. (2.27) e (2.28) se PML].

Fim dos laços espaciais.

Início dos laços espaciais $(z, x)$;

Atualizar $D_{x}$ [Eq. (2.37) ou Eq. (2.25)].

Atualizar $D_{z}$ [Eq. (2.38) ou Eq. (2.26)].

Fim dos laços espaciais.

Início dos laços espaciais $(z, x)$;

Cálculo de $E_{x}$ e $E_{z}$ : Se o meio for magnetoóptico, Eqs. (2.41) e (2.42) são

aplicadas. Se o meio for não-linear, Eqs. (2.39) e (2.40) são calculadas iterativamente até haver convergência de $E_{x}$ e $E_{z}$.

Fim dos laços espaciais.

Fim do laço de tempo.

Gravação dos resultados em arquivo.

Fim do algoritmo. 


\title{
APENDICE B - Algoritmo do FD-BPM bidimensional
}

\author{
Início do Algoritmo;
}

Definir discretização da malha.

Obter distribuição transversal de campo para células em $z=0$ (campo de excitação).

Calcular valores de permissividade elétrica na região não-linear para $z=0$ em função do campo de excitação [Eqs. (3.28) e (3.29)].

Início do laço espacial z (direção de propagação);

Enquanto $\operatorname{Max}\left[\frac{\left|\Psi^{m+1}-\widetilde{\Psi}^{m+1}\right|}{\left|\Psi^{m+1}\right|}\right]>\delta_{\text {tol }} \quad$ [Procedimento iterativo, Eq. (3.33)];

Calcular (ou atualizar) matrizes quadradas $\mathbf{A}(N x \times N x)$ e $\mathbf{B}(N x \times N x)$ [cada linha de A (B) engloba os coeficientes do lado esquerdo (direito) da Eq. (3.17), na qual o número da linha está relacionada ao valor de $i$, que varia de 1 a $N x]$.

Se PML não é usada, aplicar condição de contorno transparente (Seção 3.3.2).

Resolver sistema linear $\mathbf{A} \psi^{\boldsymbol{m}+\boldsymbol{1}}=\mathbf{B} \psi^{\boldsymbol{m}}$ [Eq. (3.17)] .

Atualizar valores de permissividade elétrica na região não-linear para $z$ atual em função do campo de excitação [Eqs. (3.28) e (3.29)].

Recalcular $\operatorname{Max}\left[\left|\Psi^{m+1}-\widetilde{\Psi}^{m+1}\right| /\left|\Psi^{m+1}\right|\right]$.

Fim do enquanto.

Fim do laço espacial $z$.

Gravação dos resultados em arquivo.

Fim do algoritmo. 


\section{APÊNDICE C - Algoritmo do FD-BPM 3D vetorial}

\section{Início do Algoritmo;}

Definir discretização da malha.

Obter distribuição transversal de campo para células em $z=0$ (campo de excitação).

Calcular valores de permissividade elétrica na região não-linear para $z=0$ em função do campo de excitação [Eq. (4.37)].

Início do laço espacial $z$ (direção de propagação);

$$
\begin{gathered}
\mid \text { Enquanto } \operatorname{Max}\left[\frac{\left|\Psi_{x}^{K+\frac{1}{2}}-\widetilde{\Psi}_{x}^{K+\frac{1}{2}}\right|}{\left|\Psi_{x}^{K+\frac{1}{2}}\right|}\right]>\delta_{t o l} \quad \text { (Procedimento iterativo); } \\
\widetilde{\Psi}_{x}^{K+\frac{1}{2}}=\Psi_{x}^{K+\frac{1}{2}}
\end{gathered}
$$

Calcular valores de permissividade elétrica na região não-linear para $z=(K+1 / 2) \delta z$, considerando o campo $\widetilde{\Psi}_{x}^{K+\frac{1}{2}}$ [Eqs. (4.37) e (4.38)]

Início do laço espacial $y$

Resolver equação FD-BPM (implícita em $x$ ) para a componente $\Phi_{x}^{K+\frac{1}{2}}$ [Eq. (4.35)]

Fim do laço espacial $y$

Início do laço espacial $x$

Resolver equação FD-BPM (implícita em $y$ ) para a componente $\Psi_{x}^{K+\frac{1}{2}}$ [Eq. (4.36)]

Fim do laço espacial $x$

Fim do enquanto 
$\mid \begin{gathered}\mid \text { Enquanto } \operatorname{Max}\left[\frac{\left|\Psi_{y}^{K+\frac{1}{2}}-\widetilde{\Psi}_{y}^{K+\frac{1}{2}}\right|}{\left|\Psi_{y}^{K+\frac{1}{2}}\right|}\right]>\delta_{t o l} \text { (Procedimento iterativo); } \\ \widetilde{\Psi}_{y}^{K+1}=\Psi_{y}^{K+1}\end{gathered}$

Calcular valores de permissividade elétrica na região não-linear para $z=(K+1) \delta z$, considerando o campo $\widetilde{\Psi}_{y}^{K+1}$ [Eqs. (4.37) e (4.47)]

Início do laço espacial $y$

Resolver equação FD-BPM (implícita em $x$ ) para a componente $\Phi_{y}^{K+1}[$ Eq. $(4.45)]$

Fim do laço espacial $y$

Início do laço espacial $x$

Resolver equação FD-BPM (implícita em $y$ ) para a componente $\Psi_{y}^{K+1}[\mathrm{Eq}$. (4.46)]

Fim do laço espacial $x$

Fim do enquanto

Estimar componente $E_{z}$ aplicando a lei de Gauss [Eq. (4.6)]

Fim do laço espacial $z$.

Gravação dos resultados em arquivo.

Fim do algoritmo.

Obs.: A condição de contorno usada para os limites transversais é a TBC (Seção 3.3.2), a qual foi aplicada ao cálculo de $\Phi_{x}^{K+\frac{1}{2}}, \Psi_{x}^{K+\frac{1}{2}}, \Phi_{y}^{K+1}$ e $\Psi_{y}^{K+1}$. Sua descrição foi omitida do corpo do algoritmo por motivos de compactação. 


\section{APÊNDICE D - Conversão entre simbolismos não-lineares}

$\mathrm{Na}$ literatura sobre materiais ópticos não-lineares, é comum encontrar a seguinte notação para o índice de refração:

$$
n=n_{L}+n_{2} I-n_{4} I^{2}
$$

na qual $n_{\mathrm{L}}$ (adimensional), $n_{2}\left(\mathrm{~cm}^{2} / \mathrm{GW}\right)$ e $n_{4}\left(\mathrm{~cm}^{4} / \mathrm{GW}\right)$ são constantes positivas determinando a resposta do material óptico em função da intensidade $I\left(\mathrm{GW} / \mathrm{cm}^{2}\right)$ do feixe de luz. A constante $n_{2}$ induz o efeito de autofocalização de um feixe gaussiano de alta potência propagando no meio não-linear. Entretanto, $n_{4}$, devido ao seu sinal negativo, surtirá efeito oposto, o qual se tornará relevante acima de uma determinada intensidade óptica. Isto determinará a saturação do índice de refração e equilibrará a concentração de potência no meio não-linear, evitando que o fenômeno de autofocalização catastrófica ocorra por intermédio da formação de "condensados ópticos" nestes meios.

Contudo, nos formalismos apresentados ao longo deste trabalho, optou-se por conveniência escrever a permissividade no meio em função da magnitude do campo elétrico, cuja expressão fisicamente equivalente à Eq. (D.1) pode ser escrita como

$$
\varepsilon=\varepsilon_{L}+\alpha_{2}|E|^{2}-\alpha_{4}|E|^{4}
$$

na qual $\varepsilon_{\mathrm{L}}$ (adimensional) é a parcela linear da permissividade dielétrica relativa. Os coeficientes de não-linearidade $\alpha_{2}\left(\mathrm{~m}^{2} / \mathrm{V}^{2}\right)$ e $\alpha_{4}\left(\mathrm{~m}^{4} / \mathrm{V}^{4}\right)$ exercem papéis semelhantes a $n_{2}$ e $n_{4}$, respectivamente, na Eq. (D.1).

Torna-se conveniente, portanto, encontrar a relação entre os parâmetros nãolineares destas duas últimas equações.

A intensidade pode ser escrita em função dos campos a partir do vetor de Poynting:

$$
I(t)=E(t) \times H(t)
$$


na qual, por convenção, utiliza-se a relação de onda plana no meio isotrópico linear para relacionar o campo magnético e o campo elétrico perpendiculares entre si:

$$
H(t)=\frac{E(t)}{Z}=n_{L} \frac{E(t)}{Z_{0}},
$$

tal que $Z_{0}=\sqrt{\mu_{0} / \varepsilon_{0}}$. Usando (D.4) em (D.3), obtém-se

$$
I(t)=n_{L} \frac{|E(t)|^{2}}{Z_{0}}
$$

Para uma oscilação harmônica no tempo, é conveniente usar valores RMS, $E_{R M S}=E / \sqrt{2}$, na qual $E$ é a amplitude do campo elétrico. Portanto (D.5) torna-se:

$$
I=\frac{n_{L}|E|^{2}}{2 Z_{0}}
$$

Usando (D.6), pode-se reescrever (D.2) como:

$$
\varepsilon=\varepsilon_{L}+\frac{2 \alpha_{2} Z_{0}}{n_{L}} I-\frac{4 \alpha_{4} Z_{0}^{2}}{n_{L}^{2}} I^{2}
$$

Substituindo (D.7) em $n=\sqrt{\varepsilon}$, expandindo em série de Taylor e desprezando os coeficientes de $I^{3}$ em diante, obtém-se

$$
n=\sqrt{\varepsilon_{c}^{n l}}+\frac{\alpha_{2} Z_{0}}{n_{L} \sqrt{\varepsilon_{c}^{n l}}} I-\sqrt{\varepsilon_{c}^{n l}}\left(\frac{2 \alpha_{4} Z_{0}^{2}}{n_{L}^{2} \varepsilon_{c}^{n l}}+\frac{\alpha_{2}^{2} Z_{0}^{2}}{2 n_{L}^{2}\left(\varepsilon_{c}^{n l}\right)^{2}}\right) I^{2}
$$

Comparando (D.8) e (D.1), conclui-se que

$$
\begin{aligned}
\varepsilon_{L} & =n_{L}^{2} \\
n_{2}=\frac{\alpha_{2} Z_{0}}{n_{L}^{2}} & \rightarrow \alpha_{2}=\frac{n_{L}^{2}}{Z_{0}} n_{2} \\
n_{4}=\left(\frac{2 \alpha_{4}}{n_{L}^{3}}+\frac{\alpha_{2}^{2}}{2 n_{L}^{5}}\right) Z_{0}^{2} & \rightarrow \alpha_{4}=\frac{n_{L}^{2}}{2 Z_{0}^{2}}\left(n_{L} n_{4}-\frac{n_{2}^{2}}{2}\right)
\end{aligned}
$$

Por exemplo, para $n_{0}=1.8, n_{2}=2 \times 10^{-3} \mathrm{~cm}^{2} / \mathrm{GW}$ e $n_{4}=2 \times 10^{-4} \mathrm{~cm}^{4} / \mathrm{GW}^{2}$ [59], obtém-se $\varepsilon_{L}=3,24, \alpha_{2}=1,72 \times 10^{-18} \mathrm{~m}^{2} / \mathrm{V}^{2}$ e $\alpha_{4}=4,086 \times 10^{-35} \mathrm{~m}^{4} / \mathrm{V}^{4}$. 


\section{APÊNDICE E - Teste de precisão do método FD-BPM 2D}

Considere o guia simples de três camadas linear e isotrópico, cuja seção transversal e valores de índice de refração são mostrados na Fig. E.1. A relação de dispersão (solução analítica) para o modo TM fundamental (e único para o comprimento de onda $\lambda=1,32 \mu \mathrm{m}$ ) fornece o valor de índice efetivo modal neff $0=$ 2,25330456055965, o qual será adotado como valor de referência para comparação em relação ao valor obtido através do método FD-BPM bidimensional.

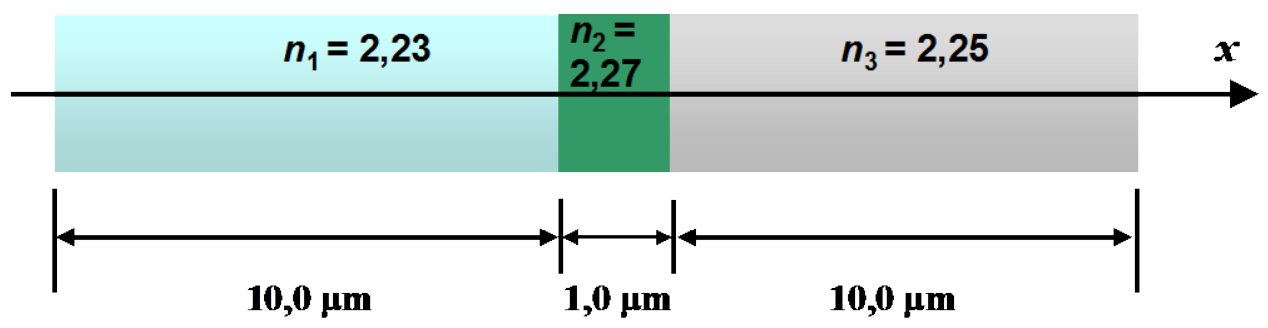

Fig. E.1 - Guia planar linear e isotrópico, $x$ é o eixo transversal.

$\mathrm{Na}$ simulação com o método FD-BPM 2D (esquema $\mathrm{CN}$ ) o modo TM foi propagado ao longo de uma distância longitudinal de $L_{z}=500 \mu \mathrm{m}$, utilizando um passo de propagação $\delta z=0,1 \mu \mathrm{m}$. A condição de contorno aplicada é a TBC.

A Fig. E.2 ilustra o erro relativo do índice efetivo, o qual foi calculado através de $\operatorname{Erro}(\%)=100 \times\left(\right.$ neff $_{B P M}-$ neff $\left._{0}\right) /$ neff $_{0}$, em função do passo transversal $\delta x$, mostrando que o método FD-BPM pode ser utilizado para calcular o índice efetivo com precisão satisfatória. A Fig. E.3 mostra os valores exatos de índice efetivo calculados através do método FD-BPM, onde é possível ter uma noção do número de casas decimais que podem ser considerados com confiabilidade, dependendo do passo de discretização transversal. A semelhança com o resultado analítico pode chegar até a quinta casa decimal para as discretizações mais finas. 


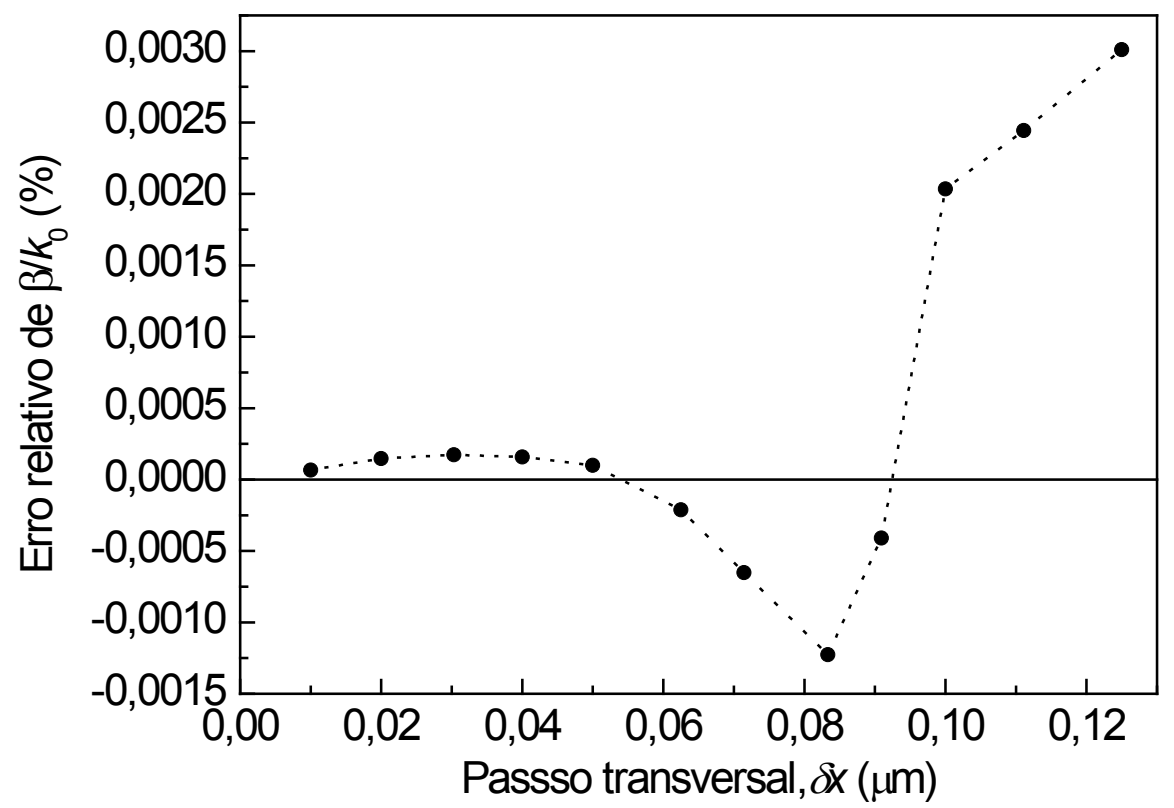

Fig. E.2 - Erro relativo do índice efetivo (FD-BPM 2D) em função do passo transversal $\delta x$.

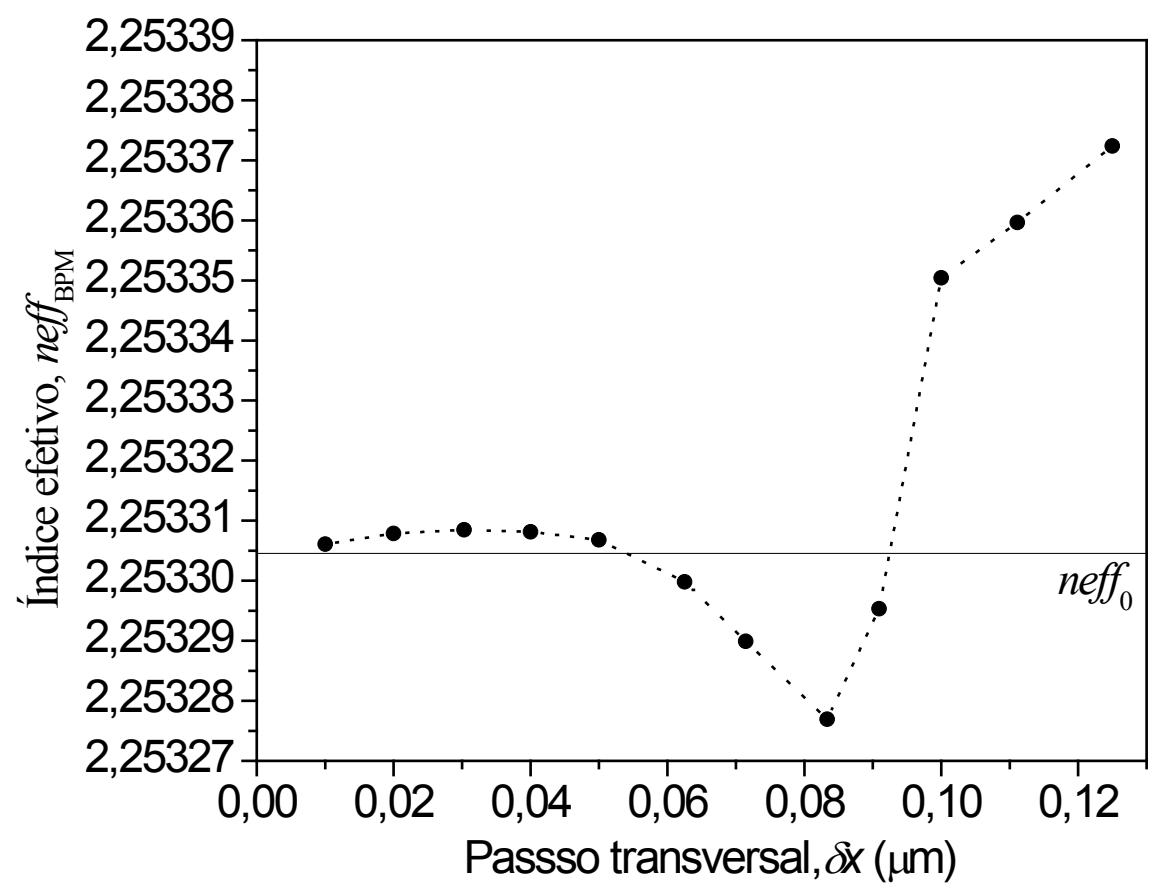

Fig. E.2 - Índice efetivo do modo TM (FD-BPM 2D) em função do passo transversal $\delta x$. 


\section{APÊNDICE F - Teste do método FD-BPM 3D vetorial}

A fim de analisar a confiabilidade da característica vetorial do método FDBPM 3D, considere uma fibra isotrópica simples, com detalhes de discretização mostrados na Fig. F.1.

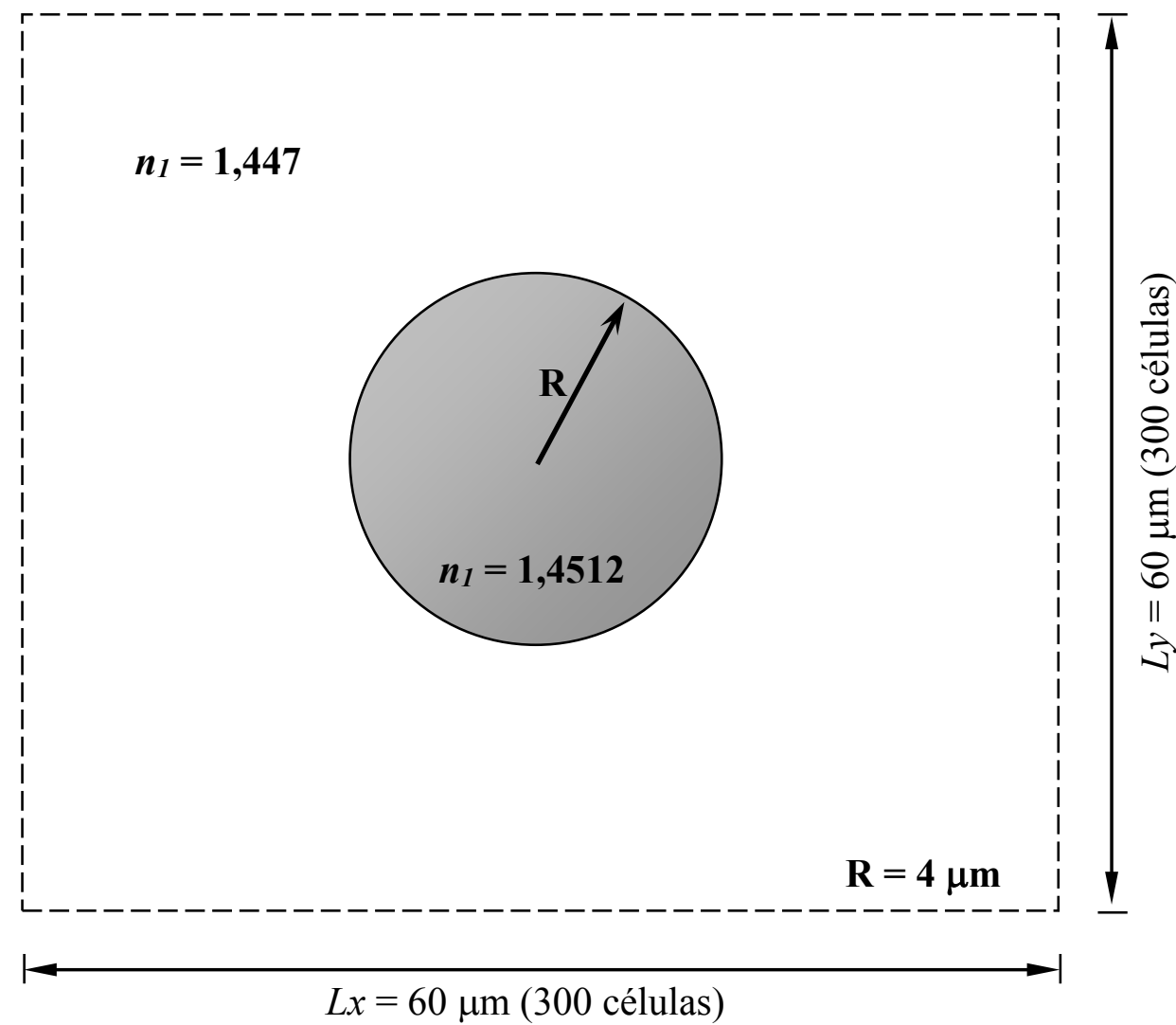

Fig. F.1 - Fibra óptica simples. Os valores dos passos de discretização são $\delta x=\delta y=\delta z=0,2 \mu \mathrm{m}$.

Analogamente ao realizado em [fl], uma simulação numérica foi posta em prática para extrair a configuração de campo elétrico do modo híbrido quasi-TE no comprimento de onda $\lambda=1,26 \mu \mathrm{m}$, o que é feito após a excitação propagar uma distância suficiente para os campos assumirem uma forma estável. A componente $E_{y}$ foi excitada (com $E_{x}=0$ ) por meio do método de sobre-relaxação sucessiva (SOR). A Fig. F.2 ilustra a configuração obtida na posição longitudinal $z=1,5 \mathrm{~mm}$. 

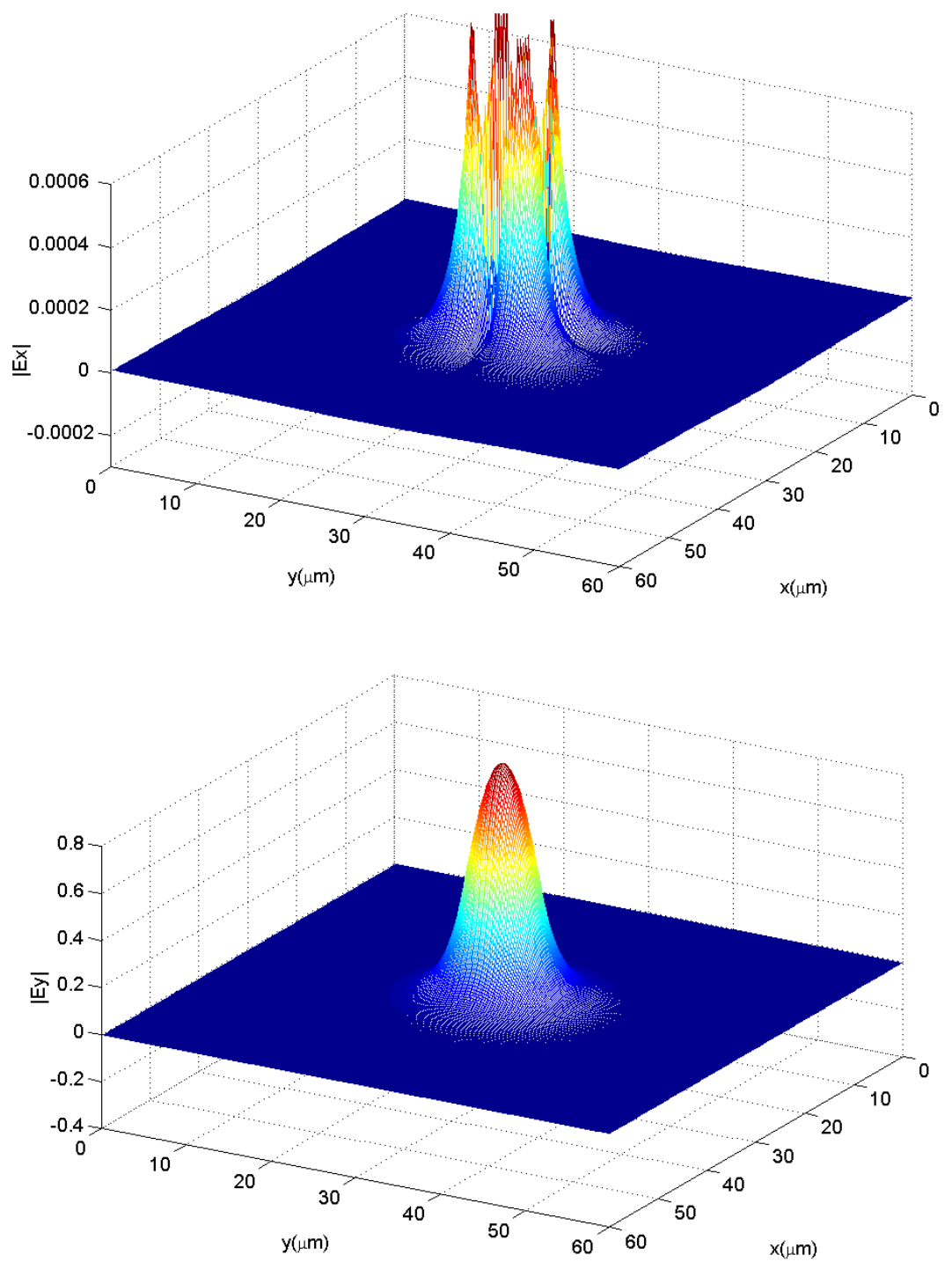

(b)

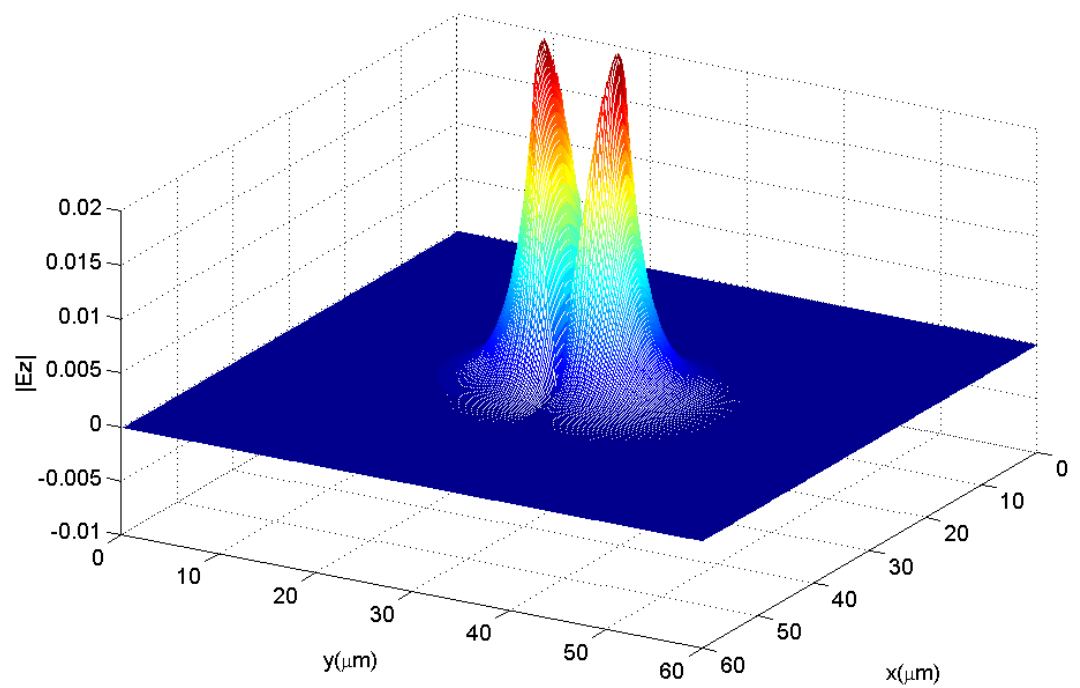

Fig. F.2 - Configuração de campo quasi-TE obtida com o método FD-BPM 3D vetorial: (a) componente $\left|E_{x}\right|$, (b) componente $\left|E_{y}\right|$ e (c) componente $\left|E_{z}\right|$. 
Os formatos e as amplitudes das distribuições mostradas na Fig. F.2 concordam bem com os resultados encontrados em [fl]. Isto vale, inclusive, para a componente $E_{z}$, a qual é calculada por meio da lei de Gauss, utilizando o índice efetivo computado (dependendo, portanto, da precisão deste). $\mathrm{O}$ valor de índice efetivo calculado para o modo é $\beta / \mathrm{k}_{0}=1,449000641$.

Observa-se também uma certa semelhança do modo da Fig. F.2. com a configuração do feixe supergaussiano de alta intensidade que se propaga no meio não-linear com saturação (Fig. 25). Na Fig. F2, a relação de amplitudes entre a componente transversal minoritária (no caso, $E_{x}$ ) e a majoritária (no caso, $E_{y}$ ) é de $\left|E_{x}\right|_{\max } /\left|E_{y}\right|_{\max }=0,00100544881367$. A relação entre a componente longitudinal e a transversal majoritária é de $\left|E_{x}\right|_{\max } /\left|E_{y}\right|_{\max }=0,03140767630549$. Esses valores são equiparáveis às relações mostradas na TABELA 9 a TABELA 16, para o caso dos condensados ópticos.

\section{Referência:}

[fl] F. Fogli, G. Bellanca, P. Bassi, I. Madden, and W. Johnstone, "Highly efficient full-vectorial 3-D BPM modeling of fiber to planar waveguide couplers," $J$. Lightwave Technol., vol. 17, no. 1, pp. 136-146, Janeiro 1999. 